Fórmula de aproximação de Baouendi-Treves

\author{
Luís Márcio Salge
}



Assinatura:

\section{Luís Márcio Salge}

\section{Fórmula de aproximação de Baouendi-Treves}

Dissertação apresentada ao Instituto de Ciências Matemáticas e de Computação - ICMC-USP, como parte dos requisitos para obtenção do título de Mestre em Ciências - Matemática. VERSÃO REVISADA

Área de Concentração: Matemática

Orientador: Prof. Dr. Éder Ritis Aragão Costa 
Ficha catalográfica elaborada pela Biblioteca Prof. Achille Bassi e Seção Técnica de Informática, ICMC/USP, com os dados fornecidos pelo(a) autor(a)

Salge, Luís Márcio
Fórmula de aproximação de Baouendi-Treves / Luís
Márcio Salge; orientador Éder Ritis Aragão Costa. --
São Carlos, 2015.
143 p.
Dissertação (Mestrado - Programa de Pós-Graduação
em Matemática) -- Instituto de Ciências Matemáticas
e de Computação, Universidade de São Paulo, 2015.
1. fórmula de baouendi-treves. 2. estruturas
localmente integráveis. 3. distribuições . I. Ritis
Aragão Costa, Éder , orient. II. Título.




\section{Luís Márcio Salge}

\section{Baouendi-Treves approximation formula}

Master dissertation submitted to the Instituto de Ciências Matemáticas e de Computação - ICMCUSP, in partial fulfillment of the requirements for the degree of the Master Program in Mathematics. FINAL VERSION

Concentration Area: Mathematics

Advisor: Prof. Dr. Éder Ritis Aragão Costa 



\section{Agradecimentos}

Inicialmente gostaria de agradecer em especial ao meu orientador de mestrado Prof. Dr. Éder Ritis Aragão Costa e aos meus orientadores durante a graduação Prof. Dr. Adalberto Panobianco Bergamasco e Profa. Dra. Roberta Godoi Wik Atique pela amizade, dedicação e paciência durante a minha formação.

Aos meus pais, tios, demais familiares e amigos pelo seu amor e comprenssão nos momentos mais difíceis da minha formação. Em especial aos meus avós paternos Márcio Antônio Salge e Terezinha Lemos Salge que me criaram, educaram e me ajudaram a chegar onde estou.

Aos professores e funcionários do Instituto de Ciências Matemáticas e de Computação que contribuíram na minha formação durante a graduação e o mestrado.

À CAPES e Fapesp pelo apoio financeiro, sem os quais esse projeto não teria sido possível.

Peço desculpa às pessoas não citadas e contribuíram direta ou indiretamente na minha formação. 

"Os loucos abrem os caminhos que depois emprestam aos sensatos" (Carlo Dossi) 



\section{Resumo}

O objetivo principal de estudo deste trabalho são as estruturas localmente integráveis $\mathcal{L}$ e a fórmula de aproximação de Baouendi-Treves, segundo a qual qualquer solução homogênea de

$$
\mathcal{L} u=0,
$$

pode, localmente, ser aproximada por polinômios nas suas integrais primeiras.

A realização deste projeto requer um estudo rigoroso de alguns aspectos da teoria das estruturas involutivas e da teoria das distribuições. As princiapais referências são [2], [4] e [1].

Palavras-chave: fórmula de Baouendi-Treves. estruturas localmente integráveis. distribuições. 



\section{Abstract}

The main goal of this project is to study a locally integrable structures $\mathcal{L}$ and the Baouendi-Treves approximation formula, which states that every homogeneous solution of

$$
\mathcal{L} u=0,
$$

can be, locally, approximated by polynomials in their first integrals.

This result requires the rigorous study of some aspects of the involutive structures theory and of the distributions theory. The main references are [2], [4] e $[1]$.

Key-words: Baouendi-Treves approximation formula. locally integrable structures. distributions. 



\section{Sumário}

Introdução $\ldots \ldots \ldots \ldots \ldots \ldots \ldots \ldots \ldots \ldots \ldots \ldots \ldots \ldots \ldots \ldots$

1 Campos Vetoriais Complexos . . . . . . . . . . . . . . 19

1.1 Variedades Diferenciáveis $N$-Dimensionais . . . . . . . . . . . . . . . 19

1.2 Funções Suaves Definidas em Uma Variedade . . . . . . . . . . . . . . . 21

1.3 Campos Vetoriais Complexos . . . . . . . . . . . . . . 21

2 Estrutura Algébrica de $\mathfrak{X}(\Omega) \ldots \ldots \ldots \ldots \ldots \ldots \ldots \ldots$

2.1 Representação Local de Campos Vetoriais . . . . . . . . . . . . . . 25

3 Estruturas Involutivas (ou Formalmente Integráveis) $\ldots \ldots . \ldots 29$

3.1 Germes de Funções $C^{\infty} \ldots \ldots \ldots \ldots$

3.2 Fibrado Tangente Complexificado $\mathbb{C} T \Omega \ldots \ldots \ldots \ldots \ldots$

3.3 Subfibrado Vetorial Complexo $\mathcal{V} \subset \mathbb{C} T \Omega \ldots \ldots \ldots \ldots . \ldots \ldots$

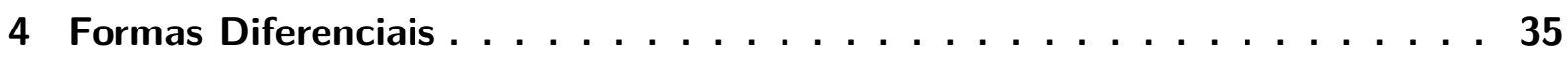

4.1 Fibrado Cotangente Complexificado $\mathbb{C} T^{*} \Omega \ldots \ldots \ldots \ldots \ldots$

4.2 Subfibrado Vetorial Complexo $\mathcal{W} \subset \mathbb{C} T^{*} \Omega \ldots \ldots \ldots \ldots$

4.3 Conjugação Complexa . . . . . . . . . . . . . . . . . . 41

5 Teorema de Frobenius $\ldots \ldots \ldots \ldots \ldots \ldots \ldots \ldots \ldots$

5.1 Problema de Cauchy . . . . . . . . . . . . . . . . . . . 43

5.2 Teorema de Frobenius $\ldots \ldots \ldots \ldots \ldots \ldots$

6 Estruturas Localmente Integráveis . . . . . . . . . . . . 61

6.1 Conjunto Característico . . . . . . . . . . . . . . . 61

6.2 Algumas estruturas especiais . . . . . . . . . . . . . . 65

6.3 Estruturas Localmente Integráveis . . . . . . . . . . . . . . 75

6.4 Geradores Locais . . . . . . . . . . . . . . . . . 78

7 Fórmula de Aproximação de Baouendi-Treves . . . . . . . . . . . . 111

7.1 Versão Clássica . . . . . . . . . . . . . . . . . . . . . . 111

7.2 Versão Distribucional . . . . . . . . . . . . . . . . . . . . . . . 118

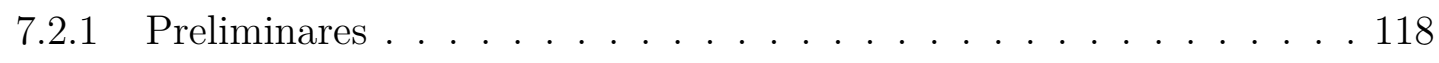

7.2 .2 Teorema de Baouendi-Treves . . . . . . . . . . . . . . . . 137

Referências . . . . . . . . . . . . . . . . . . 143 



\section{Introdução}

O foco deste estudo são as estruturas involutivas localmente geradas por campos vetoriais complexos e o Teorema de Aproximação de Bouendi-Treves, o qual é uma ferramenta fundamental no estudo de sistemas involutivos de campos vetoriais complexos. Tal teorema fornece aproximações de soluções homogêneas de estruturas localmente integráveis em espaços de funções, tais como os espaços $L^{p}$, dessa forma possui aplicações em equações diferenciais parciais e várias variáveis complexas.

Neste estudo, sistemas de vetores complexos surgem como sendo uma base local de subfibrados vetoriais complexos involutivos $\mathcal{V}$ do fibrado tangente complexificado $\mathbb{C} T \Omega$. Dizer que $\mathcal{V}$ é involutiva significa que o colchete de Lie de quaisquer duas seções de $\mathcal{V}$ também é, por sua vez, uma seção de $\mathcal{V}$.

Alguns exemplos de estruturas involutivas $(\Omega, \mathcal{V})$ incluem estruturas complexas, estruturas $C R$ (ou de Cauchy-Riemann), estruturas localmente integráveis e foliações.

Para compreender o funcionamento destas estruturas, iniciamos com o estudo de campos vetoriais complexos definidos em uma variedade suave $\Omega$. Em seguida, passamos a estudar subfibrados vetoriais complexos $\mathcal{V}$ do fibrado tangente complexificado $\mathbb{C} T \Omega$, que são basicamente uma coleção de espaços vetoriais de mesma dimensão $\left\{\mathcal{V}_{p}\right\}_{p \in \Omega}$ e que são localmente gerados por campos vetoriais complexos.

Com isso podemos definir o que são estruturas involutivas. O Teorema de Frobenius é de suma importância para tal tema, para este teorema apresentamos uma demonstração detalhada tanto na versão que envolve um aberto de $\mathbb{R}^{N}$, quanto para a versão que envolve uma variedade suave $N$-dimensional.

Em sequência, estudamos algumas estruturas especiais tais como estruturas complexas, essencialmente reais, CR, elíticas. Sendo que existe uma espécie de equivalência entre a definição de uma estrutura seja elítica e o fato de os campos que geram localmente tal estrutura serem elíticos no sentido de operadores diferenciais parciais lineares. O conceito de conjunto característico $T^{0}$ de uma estrutura $\mathcal{V}$ também desempenha um papel importante no estudo de estruturas involutivas.

Daí em diante, nos focamos no estudo de estruturas localmente integráveis $\mathcal{V}$, em que o subfibrado vetorial complexo $\mathcal{V}^{\perp}$ do fibrado cotangente complexificado $\mathbb{C} T^{*} \Omega$ é localmente gerado por diferenciais exatas.

Quando $(\Omega, \mathcal{V})$ é uma estrutura localmente integrável, cada ponto de $\Omega$ possui uma vizinhança $U$ tal que se $\left\{L_{1}, \ldots, L_{n}\right\}$ forem $n$ campos vetoriais com

$$
\mathcal{V}_{p}=\left[L_{1 p}, \ldots, L_{n p}\right], p \in U,
$$


então neste caso podemos encontrar $m=N-n$ funções $Z_{1}, \ldots, Z_{m} \in C^{\infty}(U ; \mathbb{C})$, que são soluções de

$$
L_{j} h=0, j=1, \ldots, n
$$

e cujas diferenciais são linearmente independentes sobre $\mathbb{C}$ para quaisquer pontos de $U$. Chamamos as funções $Z=\left(Z_{1}, \ldots, Z_{m}\right)$ de conjunto completo de integrais primeiras na vizinhança $U$.

Por fim, apresentamos e demonstramos a Fórmula de Baouendi-Treves que afirma que qualquer $u \in \mathscr{D}^{\prime}(W)$ solução de 1 pode ser aproximada, localmente, por uma sequência $P_{k}(Z)$, em que $P_{k}$ são polinômios holomorfos de $m$ variáveis e $Z=\left(Z_{1}, \ldots, Z_{m}\right)$ é um conjunto completo de integrais primeiras.

A Fórmula de Baouendi-Treves está dividida nas versões clássica, em que a solução $u$ é uma função suave, e distribucional. Destacamos que na demonstração da versão clássica fizemos uso de resultados de cálculo para funções de várias variáveis, matrizes hermitianas simétricas com parte real positivo definida, Teorema de Stokes para formas diferenciais, pull-back de formas diferenciais e análise complexa. Já no caso distribucional foram necessários conceitos mais sofisticados, tais como conjunto frente de onda, o estudo de subconjuntos densos de funções regulares em espaços do tipo

$$
C_{c}^{0}\left(\left\{t \in \mathbb{R}^{n} ;|t| \leq T\right\} ; H^{k}\left(\mathbb{R}^{m}\right)\right),
$$

propriedades do operador laplaciano, dentre outros.

Ressaltamos ainda, que tal teorema de aproximação tornou possível a utilização de ferramentas de análise complexa, análise harmônica e de equações diferenciais parciais no estudo de problemas envolvendo estruturas localmente integráveis. A saber, problemas sobre regularidade local e microlocal das soluções de 1, a determinação de conjuntos de unicidade para soluções de 1, resolubilidade de complexos diferenciais associados a estrutura $(\Omega, \mathcal{V})$, dentre outros. 


\section{Campos Vetoriais Complexos}

\subsection{Variedades Diferenciáveis $N$-Dimensionais}

Inicialmente, introduzimos o conceito de uma variedade diferenciável. Uma variedade diferenciável nada mais é do que um espaço topológico munido de uma estrutura diferenciável que nos permite utilizar ferramentas do cálculo diferencial para estudar funções definidas nestes espaços.

Definição 1.1.1 (Estrutura Diferenciável Sobre $\Omega$ ). Seja $\Omega$ um espaço topológico, Hausdorff e segundo enumerável. Considere a coleção $\mathcal{F} \doteq\{(U, x)\}$, onde $U \subset \Omega$ é um aberto não vazio,

$$
x: U \rightarrow \mathbb{R}^{N}
$$

é um homeomorfismo sobre o aberto $x(U)$ de $\mathbb{R}^{N}$ e as seguintes propriedades são satisfeitas:

1. $\cup_{(U, x) \in \mathcal{F}} U=\Omega$;

2. a aplicação

$$
x^{\prime} \circ x^{-1}: x\left(U \cap U^{\prime}\right) \rightarrow x^{\prime}\left(U \cap U^{\prime}\right)
$$

é infinitamente diferenciável para cada par $(U, x),\left(U^{\prime}, x^{\prime}\right) \in \mathcal{F}$ tais que $U \cap U^{\prime} \neq \emptyset$.

3. $\mathcal{F}$ é maximal com respeito as duas propriedades acima, ou seja, se $(V, y)$ são tais que $V \subset \Omega$ é um aberto não vazio, $y: V \rightarrow y(V)$ um homeomorfismo sobre o aberto $y(V)$ de $\mathbb{R}^{N}$ e para cada $(U, x) \in \mathcal{F}$, tal que $U \cap V \neq \emptyset$, as aplicações

$$
\begin{aligned}
& y \circ x^{-1}: x(U \cap V) \rightarrow y(U \cap V) \\
& x \circ y^{-1}: y(U \cap V) \rightarrow x(U \cap V)
\end{aligned}
$$

são infinitamente diferenciáveis, então $(y, V) \in \mathcal{F}$.

Tal coleção $\mathcal{F}$ é chamada de estrutura diferenciável de dimensão $N$ sobre $\Omega$.

Observação 1.1.2. Se $\mathcal{F}^{*}=\{(U, x)\}$ é uma coleção que satisfaz as propriedades 1 e 2 da definição acima, então existe única estrutura diferenciável $\mathcal{F}$ de dimensão $N$ sobre $\Omega$ tal que $\mathcal{F}^{*} \subset \mathcal{F}$.

De fato, defina a família $\mathcal{F}=\mathcal{F}^{*} \cup \mathcal{A}$, onde a coleção $\mathcal{A}$ é formada pelos pares $(y, V)$ tais que $V \subset \Omega$ é um aberto não vazio, $y: V \rightarrow y(V)$ um homeomorfismo sobre o 
aberto $y(V)$ de $\mathbb{R}^{N}$ e para cada $(U, x) \in \mathcal{F}^{*}$, tal que $U \cap V \neq \emptyset$, as aplicações

$$
\begin{aligned}
& y \circ x^{-1}: x(U \cap V) \rightarrow y(U \cap V) \\
& x \circ y^{-1}: y(U \cap V) \rightarrow x(U \cap V)
\end{aligned}
$$

são suaves. Observe que $\mathcal{F}^{*} \subset \mathcal{F}$.

Além disso, pela forma como $\mathcal{F}$ foi definida, tal coleção satisfaz as propriedades 1,2 e 3, pois se $(y, V)$ tais que $V \subset \Omega$ é um aberto não vazio, $y: V \rightarrow y(V)$ um homeomorfismo sobre o aberto $y(V)$ de $\mathbb{R}^{N}$ e para cada $(U, x) \in \mathcal{F}$, em particular para cada $(U, x) \in \mathcal{F}^{*}$ tal que $U \cap V \neq \emptyset$, as aplicações

$$
\begin{aligned}
& y \circ x^{-1}: x(U \cap V) \rightarrow y(U \cap V) \\
& x \circ y^{-1}: y(U \cap V) \rightarrow x(U \cap V)
\end{aligned}
$$

são suaves, então $(y, V) \in \mathcal{F}$. Logo esta coleção define uma estrutura diferenciável de dimensão $N$ sobre $\Omega$. Se $\mathcal{G}$ for outra estrutura diferenciável de dimensão $N$ sobre $\Omega$ que contém $\mathcal{F}^{*}$, então sendo $\mathcal{G}$ maximal tem-se $\mathcal{F} \subset \mathcal{G}$, sendo $\mathcal{F}$ maximal tem-se $\mathcal{G} \subset \mathcal{F}$ e $\operatorname{assim} \mathcal{G}=\mathcal{F}$.

Definição 1.1.3 (Variedade Diferenciável (Suave) de Dimensão $N$ ). Seja $\Omega$ um espaço topológico, Hausdorff e segundo enumerável, o qual é munido de uma estrutura diferenciável $\mathcal{F}$ de dimensão $N$. Dizemos que o par $(\Omega, \mathcal{F})$ é uma variedade diferenciável de dimensão $N$.

Por simplicidade, denotamos a variedade diferenciável $(\Omega, \mathcal{F})$ de dimensão $N$ por $\Omega$. Se, na definição 1.1.1, exigíssemos que as funções fossem reais analíticas, ou seja, infinitamente diferenciáveis com série de potências convergente numa vizinhança de cada ponto de seu domínio, obteríamos uma variedade real analítica de dimensão $N$.

A seguir apresentamos alguns exemplos de variedades diferenciáveis.

Exemplo 1.1.4. $\quad$ 1. Considere $\Omega \doteq \mathbb{R}^{N}$ munido da estrutura diferenciável $\mathcal{F}$ $N$-dimensional que contém $\left(\mathbb{R}^{N}, i d\right)$, tem-se que $\Omega$ define uma variedade diferenciável $N$-dimensional;

2. Seja $\Omega$ uma variedade diferenciável $N$-dimensional munido da estrutura diferencial $\mathcal{F}=\{(U, x)\} N$-dimensional. Considere $W \subset \Omega$, aberto não vazio, munido da topologia induzida pela topologia de $\Omega$ e a coleção

$$
\mathcal{F}_{W} \doteq\left\{\left(W \cap U,\left.x\right|_{W \cap U}\right) ;(U, x) \in \mathcal{F}, W \cap U \neq \emptyset\right\} .
$$

Como Hausdorff e segundo enumerável são propriedades hereditárias, $W$ será um espaço topológico, Hausdorff e segundo enumerável. Além disso, podemos facilmente mostrar que $\mathcal{F}_{W}$ define uma estrutura diferenciável $N$-dimensional. Sendo assim $\left(W, \mathcal{F}_{W}\right)$ é uma variedade diferenciável $N$-dimensional. 
As estruturas diferenciáveis $\mathcal{F}$ também são chamadas de atlas $C^{\infty}$, seus elementos $(U, x) \in \mathcal{F}$ são chamados de cartas locais ou sistema de coordenadas local. Escrevemos $x=\left(x_{1}, \ldots, x_{N}\right)$, assim $p \in U$ tem suas coordenadas locais dadas por $x(p)=$ $\left(x_{1}(p), \ldots, x_{N}(p)\right)$.

\subsection{Funções Suaves Definidas em Uma Variedade}

Nesta seção, a partir da definição de variedade diferenciável introduzida na seção anterior, definimos o conceito de função suave definida em uma variedade diferenciável.

Definição 1.2.1 (Funções Suaves Definidas em uma Variedade). Uma função $f: \Omega \rightarrow \mathbb{C}$ é dita suave, ou infinitamente diferenciável se para cada $(U, x) \in \mathcal{F}$, tivermos que

$$
f \circ x^{-1}: x(U) \rightarrow \mathbb{C}
$$

é infinitamente diferenciável. O conjunto constituído por tais funções é denotado por $C^{\infty}(\Omega)$.

Observação 1.2.2. Se $f: \Omega \rightarrow \mathbb{C}$ é tal que, para cada $p \in \Omega$, existe $(U, x) \in \mathcal{F}$ com $p \in U$ e $f \circ x^{-1} \in C^{\infty}(x(U))$, então $f \in C^{\infty}(\Omega)$.

De fato, seja $(V, y) \in \mathcal{F}$ com $V \neq \emptyset$. Vamos mostrar que $f \circ y^{-1} \in C^{\infty}(y(V))$. Para tanto, basta que para cada $p \in V$ exista aberto $W \subset y(V)$, que contém $y(p)$, tal que $\left.f \circ y^{-1}\right|_{W} \in C^{\infty}(W)$. Seja $p \in V$, note que existe $(x, U) \in \mathcal{F}$, com $p \in U$, tal que $f \circ x^{-1} \in C^{\infty}(x(U))$. Como $x \circ y^{-1}$ é suave, então $\left.f \circ y^{-1}\right|_{W}=\left(f \circ x^{-1}\right) \circ\left(x \circ y^{-1}\right)$ é suave.

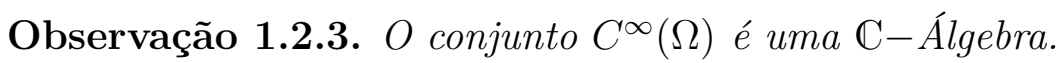

\subsection{Campos Vetoriais Complexos}

Dada uma variedade diferenciável $\Omega$ de dimensão $N$, vamos introduzir o conceito de campo vetorial complexo que é uma aplicação $\mathbb{C}$-linear definida no conjunto das funções suaves $C^{\infty}(\Omega)$, tomando valores neste mesmo conjunto $C^{\infty}(\Omega)$ e que satisfaz uma certa propriedade, que será especificada mais adiante, de forma que estas aplicações se comportam essencialmente como derivadas de primeira ordem. O conceito de campos vetoriais é relevante no nosso contexto pois facilita o estudo da convergência de sequências de funções e suas derivadas.

Definição 1.3.1 (Campos Vetoriais Complexos). Um campo vetorial complexo (suave) é uma aplicação $\mathbb{C}$-linear:

$$
L: C^{\infty}(\Omega) \rightarrow C^{\infty}(\Omega)
$$


que satisfaz a regra de Leibniz, a saber,

$$
L(f g)=f L(g)+g L(f), \forall f, g \in C^{\infty}(\Omega) .
$$

Denotamos o espaço de tais objetos por $\mathfrak{X}(\Omega)$.

Proposição 1.3.2. Seja $L \in \mathfrak{X}(\Omega)$. Se f for uma função constante, então $L f=0$. Além disso,

$$
\operatorname{supp}(L f) \subset \operatorname{supp}(f), \forall f \in C^{\infty}(\Omega)
$$

Demonstração. Dado $L \in \mathfrak{X}(\Omega)$, seja $f: \Omega \rightarrow \mathbb{C}$, dada por $f(p)=c, \forall p \in \Omega$, onde $c \in \mathbb{C}$. Como $L$ é $\mathbb{C}$-linear, tem-se que:

$$
L(f)=L(1 c)=c L(1)
$$

agora basta mostrarmos que $L(1)=0$.

Pela regra de Leibniz tem-se:

$$
L(1)=L(1.1)=1 L(1)+1 L(1)=2 L(1)
$$

então $L(1)=0$.

Sejam $L \in \mathfrak{X}(\Omega), f \in C^{\infty}(\Omega)$ e considere as seguintes famílias $\mathcal{F}_{f}, \mathcal{F}_{L f}$, onde $U \in \mathcal{F}_{f}$ se $U \subset \Omega$ for aberto não vazio tal que $\left.f\right|_{U}=0$, e $U \in \mathcal{F}_{L f}$ se $U \subset \Omega$ for aberto não vazio tal que $\left.L f\right|_{U}=0$.

Por definição, temos

$$
\operatorname{supp}(f)=\left(\cup_{U \in \mathcal{F}_{f}} U\right)^{c}
$$

e

$$
\operatorname{supp}(L f)=\left(\cup_{U \in \mathcal{F}_{L f}} U\right)^{c},
$$

logo basta mostrarmos que $\cup_{U \in \mathcal{F}_{f}} U \subset \cup_{U \in \mathcal{F}_{L f}} U$.

Seja $V \subset \Omega$ aberto não vazio tal que $\left.f\right|_{V}=0$ e mostremos que $\left.L f\right|_{V}=0$, ou seja, que para cada $p \in V$ tem-se $L f(p)=0$. Considere $p \in V$ e $(U, x) \in \mathcal{F} \operatorname{com} p \in U \subset V$.

Observe que $x(U) \subset \mathbb{R}^{N}$ é aberto e $\{x(p)\} \subset x(U)$ é compacto, logo existe $\phi \in$ $C_{c}^{\infty}(x(U))$ com $0 \leq \phi \leq 1$ e $\left.\phi\right|_{W}=1$, onde $W \subset x(U)$ é um aberto que contém $x(p)$.

Defina:

$$
g(q)= \begin{cases}\phi(x(q)), & q \in U \\ 0, & q \notin x^{-1}(\operatorname{supp}(\phi)) .\end{cases}
$$

Note que $g$ é suave, pois $\phi \circ x$ é suave e se $q \in U \cap\left(x^{-1}(\operatorname{supp}(\phi))\right)^{c}$ então $g(q)=0$. Além disso, tem-se $f=(1-g) f$. Com efeito, se $q \in x^{-1}(\operatorname{supp}(\phi)) \subset U \subset V$ então

$$
(1-g(q)) f(q)=(1-1) f(q)=0=f(q),
$$


e se $q \notin x^{-1}(\operatorname{supp}(\phi))$, consequentemente

$$
(1-g(q)) f(q)=1 f(q)=f(q) .
$$

Portanto, pela regra de Leibniz obtém-se que:

$$
\begin{aligned}
L(f)(q) & =f(q) L(1-g)(q)+(1-g(q)) L(f)(q) \\
& =L(f)(q)-g(q) L(f)(q)-f(q) L(g)(q)
\end{aligned}
$$

e assim para $q=p$

$$
L(f)(p)=L(f)(p)-L(f)(p)=0 .
$$

Como $p \in V$ é arbitrário, concluímos que $\left.L f\right|_{V}=0$ e segue que $\operatorname{supp}(L f) \subset \operatorname{supp}(f)$.

Operadores com a propriedade 1.2 são chamados de operadores locais. Com o resultado da Proposição 1.3 somos capazes de definir a restrição de um campo $L \in \mathfrak{X}(\Omega)$ a um aberto não vazio $W \subset \Omega$. Mais precisamente, definimos uma aplicação $\mathbb{C}$-linear:

$$
\mathfrak{X}(\Omega) \ni L \mapsto L_{W} \in \mathfrak{X}(W),
$$

que torna o seguinte diagrama comutativo:

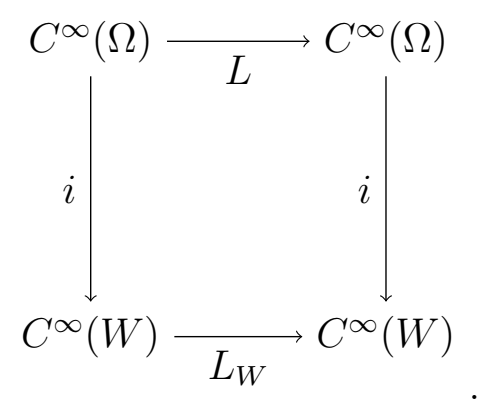

Sejam $W \subset \Omega$ um aberto não vazio e $L \in \mathfrak{X}(\Omega)$. Para cada $f \in C^{\infty}(W)$ e cada $p \in \Omega$ defina:

$$
L_{W}(f)(p) \doteq L(\tilde{f})(p)
$$

onde $\tilde{f} \in C^{\infty}(\Omega)$ é tal que $f$ coincide com $\tilde{f}$ em alguma vizinhança de $p$.

Note que $L_{W}$ está bem definido. De fato, sejam $f \in C^{\infty}(W)$ e $p \in W$, se $\hat{f}, \tilde{f} \in$ $C^{\infty}(\Omega)$ são tais que $\left.f\right|_{U}=\left.\hat{f}\right|_{U}$ e $\left.f\right|_{V}=\left.\tilde{f}\right|_{V}$, então $\left.(\hat{f}-\tilde{f})\right|_{U \cap V}=0$, ou seja, $U \cap V \subset$ $(\operatorname{supp}(\hat{f}-\tilde{f}))^{c}$.

Assim, pela Proposição 1.3 e por $L$ ser $\mathbb{C}$-linear, temos que:

$$
U \cap V \subset(\operatorname{supp} L(\hat{f}-\tilde{f}))^{c}=(\operatorname{supp}(L \hat{f}-L \tilde{f}))^{c},
$$

em outras palavras obtemos $\left.(L \hat{f}-L \tilde{f})\right|_{U \cap V}=0$ e segue que $L \hat{f}(p)=L \tilde{f}(p)$.

Além disso, podemos verificar que o operador $L_{W}$ faz o diagrama acima comutar e que de fato $L_{W} \in \mathfrak{X}(W)$. 



\section{Estrutura Algébrica de $\mathfrak{X}(\Omega)$}

Sejam $g \in C^{\infty}(\Omega)$ e $L \in \mathfrak{X}(\Omega)$ podemos definir a operação $g L$ por:

$$
\begin{aligned}
C^{\infty}(\Omega) \times \mathfrak{X}(\Omega) & \rightarrow \mathfrak{X}(\Omega) \\
(g, L) & \mapsto g L,
\end{aligned}
$$

onde $g L: C^{\infty}(\Omega) \rightarrow C^{\infty}(\Omega)$ é dada por:

$$
(g L)(f)(p) \doteq g(p) L f(p), p \in \Omega, f \in C^{\infty}(\Omega)
$$

Dessa maneira, obtemos que tal operação torna $\mathfrak{X}(\Omega)$ um $C^{\infty}(\Omega)$-módulo.

Uma operação muito relevante em $\mathfrak{X}(\Omega)$ é o Lie-Bracket ou comutador entre dois campos vetoriais que é dada por:

$$
\begin{aligned}
{[\cdot, \cdot]: \mathfrak{X}(\Omega) \times \mathfrak{X}(\Omega) } & \rightarrow \mathfrak{X}(\Omega) \\
(L, M) & \mapsto[L, M],
\end{aligned}
$$

onde $[L, M](f) \doteq L(M(f))-M(L(f)), f \in C^{\infty}(\Omega)$.

Observação 2.0.3. O conjunto $\mathfrak{X}(\Omega)$ munido do comutador é uma álgebra de Lie sobre $\mathbb{C}$, pois $[\cdot, \cdot]$ é um operador bilinear, alternado e respeita a identidade de Jacobi.

\subsection{Representação Local de Campos Vetoriais}

Considere $\Omega$ uma variedade diferenciável $N$-dimensional com estrutura diferenciável $\mathcal{F}=\{(U, x)\}$. Fixe $p \in \Omega$, sejam $(U, x)$ uma carta local de $\Omega$ com $p \in U, L \in \mathfrak{X}(U)$ e escreva:

$$
x(q)=\left(x_{1}(q), \ldots, x_{N}(q)\right), q \in U .
$$

Observe que $x(p) \in x(U) \subset \mathbb{R}^{N}$, então existe $r_{p}>0$ tal que $B\left(x(p), r_{p}\right) \subset x(U)$. Tome $V \doteq x^{-1}\left(B\left(x(p), r_{p}\right)\right) \subset U$. Defina $a=\left(a_{1}, \ldots, a_{N}\right) \doteq x(p)$, assim $p \in V$ e $x(V)=$ $B\left(a, r_{p}\right)$.

Dada $f \in C^{\infty}(U)$, tome $f^{*} \doteq f \circ x^{-1}: x(U) \rightarrow \mathbb{C}$, em particular tem-se que $f^{*} \in C^{\infty}(x(U))$.

Para cada $\left(x_{1}, \ldots, x_{N}\right) \in x(V)$, considere a função $\psi:[0,1] \rightarrow \mathbb{C}$, dada por

$$
\psi(t) \doteq f^{*}(a+t(x-a))=f^{*}\left(a_{1}+t\left(x_{1}-a_{1}\right), \ldots, a_{N}+t\left(x_{N}-a_{N}\right)\right) .
$$


Assim $\psi \in C^{\infty}([0,1])$ e podemos aplicar o Teorema Fundamental do Cálculo para obter:

$$
\psi(1)-\psi(0)=\int_{0}^{1} \psi^{\prime}(t) d t
$$

Portanto:

$$
f^{*}\left(x_{1}, \ldots, x_{N}\right)=f^{*}\left(a_{1}, \ldots, a_{N}\right)+\sum_{j=1}^{N} h_{j}\left(x_{1}, \ldots, x_{N}\right)\left(x_{j}-a_{j}\right),
$$

onde

$$
h_{j}\left(x_{1}, \ldots, x_{N}\right) \doteq \int_{0}^{1} \frac{\partial f^{*}}{\partial x_{j}}(a+t(x-a)) d t \in C^{\infty}(x(V))
$$

Tome $g_{j}=h_{j} \circ x \in C^{\infty}(V)$, substituindo em 2.1 tem-se que

$$
f(q)=f(p)+\sum_{j=1}^{N} g_{j}(q)\left(x_{j}(q)-x_{j}(p)\right), q \in V
$$

Logo pela regra de Leibniz concluímos

$$
L_{V}\left(\left.f\right|_{V}\right)(q)=L(f)(q)=\sum_{j=1}^{N}\left(L g_{j}\right)(q)\left(x_{j}(q)-x_{j}(p)\right)+\sum_{j=1}^{N} g_{j}(q)\left(L x_{j}\right)(q), q \in V .
$$

Por fim, avaliando em $q=p$ :

$$
L(f)(p)=\sum_{j=1}^{N} \frac{\partial f^{*}}{\partial x_{j}}(x(p)) L\left(x_{j}\right)(p) .
$$

Para cada $j=1, \ldots, N$ considere a seguinte aplicação $\mathbb{C}$-linear

$$
\begin{aligned}
C^{\infty}(U) & \rightarrow C^{\infty}(U) \\
f & \mapsto \frac{\partial f^{*}}{\partial x_{j}} \circ x
\end{aligned}
$$

a qual denotamos por $\frac{\partial}{\partial x_{j}}$, define um elemento de $\mathfrak{X}(U)$.

De 2.2 tem-se que

$$
\begin{aligned}
L(f)(p) & =\sum_{j=1}^{N} \frac{\partial f^{*}}{\partial x_{j}}(x(p))\left(L x_{j}\right)(p)= \\
& =\sum_{j=1}^{N} L\left(x_{j}\right)(p) \frac{\partial}{\partial x_{j}}(f)(p),
\end{aligned}
$$

como $p \in U$ e $f \in C^{\infty}(U)$ são arbitrários, concluímos que

$$
L=\sum_{j=1}^{N}\left(L x_{j}\right) \frac{\partial}{\partial x_{j}} .
$$

Consequentemente $\mathfrak{X}(U)$ é um $C^{\infty}(U)$-módulo livre com base

$$
\left\{\frac{\partial}{\partial x_{1}}, \ldots, \frac{\partial}{\partial x_{N}}\right\}
$$


Observação 2.1.1. Observe que para quaisquer $j, k=1, \ldots, N$ vale

$$
\begin{aligned}
\frac{\partial x_{j}}{\partial x_{k}} & =\delta_{j k}, \\
\frac{\partial}{\partial x_{j}} \circ \frac{\partial}{\partial x_{k}} & =\frac{\partial}{\partial x_{k}} \circ \frac{\partial}{\partial x_{j}} .
\end{aligned}
$$

Com efeito, note que $x_{j} \circ x^{-1}(q)=q_{j}, q=\left(q_{1}, \ldots, q_{N}\right) \in x(U)$ pois

$$
x \circ x^{-1}=\left(x_{1} \circ x^{-1}, \ldots, x_{j-1} \circ x^{-1}, x_{j} \circ x^{-1}, x_{j+1} \circ x^{-1}, \ldots, x_{N} \circ x^{-1}\right)=i d \text {, }
$$

assim, para cada $p \in U$ denote $q=x(p)$, tem-se que

$$
\begin{aligned}
\frac{\partial x_{j}}{\partial x_{k}}(p) & =\frac{\partial\left(x_{j} \circ x^{-1}\right)}{\partial e_{k}}(q)=\lim _{t \rightarrow 0} \frac{x_{j} \circ x^{-1}\left(q+t e_{k}\right)-x_{j} \circ x^{-1}(q)}{t}= \\
& =\lim _{t \rightarrow 0} \frac{q_{j}-q_{j}}{t}=0, j \neq k, \\
\frac{\partial x_{j}}{\partial x_{j}}(p) & =\frac{\partial\left(x_{j} \circ x^{-1}\right)}{\partial e_{j}}(q)=\lim _{t \rightarrow 0} \frac{x_{j} \circ x^{-1}\left(q+t e_{j}\right)-x_{j} \circ x^{-1}(q)}{t}= \\
& =\lim _{t \rightarrow 0} \frac{q_{j}+t-q_{j}}{t}=1,
\end{aligned}
$$

logo obtemos 2.3 .

Seja $f \in C^{\infty}(U)$ tem-se que

$$
\begin{aligned}
\frac{\partial}{\partial x_{j}}\left(\frac{\partial f}{\partial x_{k}}\right) & =\frac{\partial}{\partial x_{j}}\left(\frac{\partial\left(f \circ x^{-1}\right)}{\partial e_{k}} \circ x\right)= \\
& =\left(\frac{\partial}{\partial e_{j}}\left(\frac{\partial\left(f \circ x^{-1}\right)}{\partial e_{k}} \circ x \circ x^{-1}\right)\right) \circ x= \\
& =\left(\frac{\partial}{\partial e_{j}}\left(\frac{\partial\left(f \circ x^{-1}\right)}{\partial e_{k}}\right)\right) \circ x= \\
& =\left(\frac{\partial}{\partial e_{k}}\left(\frac{\partial\left(f \circ x^{-1}\right)}{\partial e_{j}}\right)\right) \circ x= \\
& =\left(\frac{\partial}{\partial e_{k}}\left(\frac{\partial\left(f \circ x^{-1}\right)}{\partial e_{j}} \circ x \circ x^{-1}\right)\right) \circ x= \\
& =\frac{\partial}{\partial x_{k}}\left(\frac{\partial f}{\partial x_{j}}\right),
\end{aligned}
$$

portanto concluímos 2.4 . 



\section{Estruturas Involutivas (ou Formalmente In- tegráveis)}

Neste capítulo, apresentamos as noções de fibrado tangente complexificado $\mathbb{C} T \Omega$ e subfibrado vetorial complexo $\mathcal{V} \subset \mathbb{C} T \Omega$. Daí podemos definir o conceito de estruturas involutivas que tem um papel importante no nosso contexto.

\subsection{Germes de Funções $C^{\infty}$}

Seja $p \in \Omega$ e denote por $\mathcal{B}_{p}$ o conjunto dos pares $(V, f)$, onde $V \subset \Omega$ aberto com $p \in V$ e $f \in C^{\infty}(V)$. Defina a relação $\sim$ em $\mathcal{B}_{p}$ da seguinte forma $\left(V_{1}, f_{1}\right) \sim\left(V_{2}, f_{2}\right)$ se, e somente se, $f_{1}$ coincide com $f_{2}$ numa vizinhança de $p$.

A relação definida acima é de equivalência. Denotamos $\mathcal{B}_{p} / \sim$ por $C^{\infty}(p)$ e chamamos seus elementos, $\underline{f} \in C^{\infty}(p)$, de germes de uma função $C^{\infty}$ em $p$.

Observação 3.1.1. O conjunto $C^{\infty}(p)$ é uma $\mathbb{C}$-álgebra. Além disso, podemos conjugar as classes de germes $\underline{f} \in C^{\infty}(p)$, basta tomar um elemento $g \in \underline{f}$ e considerar $\underline{\bar{f}}$ como sendo a classe da função $\bar{g}$.

\subsection{Fibrado Tangente Complexificado $\mathbb{C} T \Omega$}

Definição 3.2.1. Um vetor complexo tangente à $\Omega$ em p é uma aplicação $\mathbb{C}$-linear

$$
v: C^{\infty}(p) \rightarrow \mathbb{C}
$$

tal que

$$
v(\underline{f g})=f(p) v(\underline{g})+g(p) v(\underline{f}), \underline{f}, \underline{g} \in C^{\infty}(p) .
$$

Denotamos por $\mathbb{C} T_{p} \Omega$ o conjunto de todos os vetores complexos tangentes à $\Omega$ em $p$, o qual é chamado de espaço tangente à $\Omega$ em p, e por $\mathbb{C} T \Omega$ a coleção $\cup_{p \in \Omega} \mathbb{C} T_{p} \Omega$, a qual é chamada de fibrado tangente complexificado.

Observação 3.2.2. Note que o conjunto $\mathbb{C} T_{p} \Omega$ é um espaço vetorial.

Observação 3.2.3. Se $L \in \mathfrak{X}(\Omega)$, defina $L_{p}: C^{\infty}(p) \rightarrow \mathbb{C}$ por:

$$
L_{p}(\underline{f}) \doteq L(f)(p)
$$

$\operatorname{assim} L_{p} \in \mathbb{C} T_{p} \Omega$. 
Além disso, pela construção feita anteriormente temos que existe uma carta local $(U, x)$ de $\Omega$ e um aberto $V \subset U$ que contém $p$ tais que

$$
f(q)=f(p)+\sum_{j=1}^{N} g_{j}(q)\left(x_{j}(q)-x_{j}(p)\right), q \in V .
$$

Portanto obtemos

$$
\begin{aligned}
v \underline{f}) & =\sum_{j=1}^{N} v\left(\underline{x_{j}}\right) g_{j}(p)=\sum_{j=1}^{N} v\left(\underline{x_{j}}\right) \frac{\partial}{\partial x_{j}}(f)(p)= \\
& =\sum_{j=1}^{N} v\left(\underline{x_{j}}\right)\left(\frac{\partial}{\partial x_{j}}\right)_{p} \underline{(f)}
\end{aligned}
$$

e concluímos que

$$
\left.v=\sum_{j=1}^{N} v \underline{\left(x_{j}\right.}\right)\left(\frac{\partial}{\partial x_{j}}\right)_{p} .
$$

Segue deste fato que o conjunto $\mathbb{C} T_{p} \Omega$ é um espaço vetorial $N$-dimensional que admite como base o conjunto

$$
\left\{\left(\frac{\partial}{\partial x_{1}}\right)_{p}, \ldots,\left(\frac{\partial}{\partial x_{N}}\right)_{p}\right\}
$$

Observação 3.2.4. Sejam $U \subset \Omega$ um aberto não vazio e $L_{1}, \ldots, L_{n} \in \mathfrak{X}(U)$. Considere as seguintes sentenças:

1. para cada $p \in U,\left\{L_{j p}\right\}_{j=1}^{n}$ é linearmente independente;

2. se $f_{1}, \ldots, f_{n} \in C^{\infty}(U)$ forem tais que

$$
\sum_{j=1}^{n} f_{j} L_{j}=0
$$

ent $\tilde{a} o \quad f_{1}, \ldots, f_{n}=0$;

3. se $\lambda_{1}, \ldots, \lambda_{n} \in \mathbb{C}$ forem tais que

$$
\sum_{j=1}^{n} \lambda_{j} L_{j}=0
$$

então $\lambda_{1}, \ldots, \lambda_{n}=0$.

Afirmamos que $1 \Rightarrow 2 \Rightarrow 3$.

Com efeito, suponha 1 e considere $f_{1}, \ldots, f_{n} \in C^{\infty}(U)$ tais que

$$
\sum_{j=1}^{n} f_{j} L_{j}=0
$$

Assim

$$
\left(\sum_{j=1}^{n} f_{j} L_{j}\right)_{p}=\sum_{j=1}^{n} f_{j}(p) L_{j p}=0
$$


e por 1 tem-se que $f_{j}(p)=0, j=1, \ldots, n, p \in U$, ou seja, $f_{j}=0, j=1, \ldots, n$.

Agora suponha 2 e sejam $\lambda_{1}, \ldots, \lambda_{n} \in \mathbb{C}$ tais que

$$
\sum_{j=1}^{n} \lambda_{j} L_{j}=0
$$

Considere

$$
\begin{aligned}
f_{j}: U & \rightarrow \mathbb{C} \\
p & \mapsto f_{j}(p),
\end{aligned}
$$

$j=1, \ldots, n, \operatorname{assim} f_{j} \in C^{\infty}(U) e$

$$
\sum_{j=1}^{n} f_{j} L_{j}=\sum_{j=1}^{n} \lambda_{j} L_{j}=0
$$

$\operatorname{Logo} \lambda_{j}=f_{j}=0, j=1, \ldots, n$.

Proposição 3.2.5. Para cada $p \in \Omega$, tem-se que:

$$
\mathbb{C} T_{p} \Omega=\left\{L_{p} ; L \in \mathfrak{X}(\Omega)\right\}
$$

Demonstração. De fato, seja $v \in \mathbb{C} T_{p} \Omega$. Por 3.1 tem-se que existem $v_{1}, \ldots, v_{N} \in \mathbb{C}$ tais que

$$
v=\sum_{j=1}^{N} v_{j}\left(\frac{\partial}{\partial x_{j}}\right)_{p} .
$$

Seja $(U, x)$ um sistema de coordenadas locais com $p \in U$. Considere

$$
g(q)= \begin{cases}\phi(x(q)), & q \in U \\ 0, & q \notin x^{-1}(\operatorname{supp}(\phi)),\end{cases}
$$

onde $\phi \in C_{c}^{\infty}(x(U)) \operatorname{com} \phi(x(p))=1$.

Defina $L: C^{\infty}(\Omega) \rightarrow C^{\infty}(\Omega)$, dada por:

$$
L f(q)= \begin{cases}g(q) \sum_{j=1}^{N} v_{j} \frac{\partial f}{\partial x_{j}}(q), & q \in U \\ 0, & q \notin x^{-1}(\operatorname{supp}(\phi)),\end{cases}
$$

para $f \in C^{\infty}(\Omega)$.

Se $f, h \in C^{\infty}(\Omega)$ e $\lambda \in \mathbb{C}$, tem-se:

$$
L(f+\lambda h)(q)= \begin{cases}g(q)\left(\sum_{j=1}^{N} v_{j} \frac{\partial f}{\partial x_{j}}(q)+v_{j} \lambda \frac{\partial h}{\partial x_{j}}(q)\right), & q \in U \\ 0, & q \notin x^{-1}(\operatorname{supp}(\phi)),\end{cases}
$$


ou seja, $L(f+\lambda h)=L f+\lambda L h$ e

$$
L(f h)(q)= \begin{cases}g(q)\left(\sum_{j=1}^{N} v_{j} h(q)\left(\frac{\partial f}{\partial x_{j}}\right)(q)+v_{j} f(q) \frac{\partial h}{\partial x_{j}}(q)\right), & q \in U \\ 0, & q \notin x^{-1}(\operatorname{supp}(\phi)),\end{cases}
$$

então $L(f h)=h L f+f L h$ e portanto $L \in \mathfrak{X}(\Omega)$.

Além disso, se $\underline{f} \in C^{\infty}(p)$ tem-se

$$
L_{p}(\underline{f})=g(p) \sum_{j=1}^{N} v_{j}\left(\frac{\partial}{\partial x_{j}}\right)_{p}(\underline{f})=\sum_{j=1}^{N} v_{j}\left(\frac{\partial}{\partial x_{j}}\right)_{p}(\underline{f})=v(\underline{f}),
$$

$\log \mathrm{O} L_{p}=v$

\subsection{Subfibrado Vetorial Complexo $\mathcal{V} \subset \mathbb{C} T \Omega$}

Definição 3.3.1. Dizemos que o conjunto $\mathcal{V}=\cup_{p \in \Omega} \mathcal{V}_{p} \subset \mathbb{C} T \Omega$ é um subfibrado vetorial complexo $n$-dimensional se satisfaz as seguintes propriedades:

1. para cada $p \in \Omega, \mathcal{V}_{p} \subset \mathbb{C} T_{p} \Omega$ é um subespaço vetorial $n$-dimensional;

2. para cada $p_{0} \in \Omega$, existem $U \subset \Omega$ aberto com $p_{0} \in U$ e $L_{1}, \ldots, L_{n} \in \mathfrak{X}(U)$ tais que, para cada $p \in U$, vale que $\mathcal{V}_{p}=\left[L_{1 p}, \ldots, L_{n p}\right]$.

Dados $\mathcal{V} \subset \mathbb{C} T \Omega$ um subfibrado vetorial complexo $n$-dimensional e $W \subset \Omega$ aberto não vazio, dizemos que $L \in \mathfrak{X}(W)$ é uma seção de $\mathcal{V}$ sobre $W$ se

$$
L_{p} \in \mathcal{V}_{p}, \forall p \in W .
$$

Definição 3.3.2 (Estrutura Involutiva). Um subfibrado vetorial complexo $\mathcal{V} \subset \mathbb{C} T \Omega$ de dimensão $n$ é uma estrutura involutiva (ou formalmente integrável) sobre $\Omega$ se satisfaz a condição de Frobenius, a saber, dados $W \subset \Omega$ aberto não vazio e $L, M \in \mathfrak{X}(W)$ duas seções de $\mathcal{V}$ sobre $W$, então $[L, M] \in \mathfrak{X}(W)$ é, por sua vez, uma seção de $\mathcal{V}$ sobre $W$.

Definição 3.3.3. Uma solução clássica de uma estrutura involutiva $\mathcal{V}$ sobre $\Omega$ é uma função $u \in C^{1}$ tal que $L u=0$, para toda seção $L$ de $\mathcal{V}$ sobre $W$, para cada $W$ aberto de $\Omega$.

Proposição 3.3.4. Sejam $\mathcal{V}$ uma estrutura involutiva $n$-dimensional sobre $\Omega$ e $p \in \Omega$. Considere $(U, x)$ um sistema de coordenadas locais, com $p \in U$, e $L_{1}, \ldots, L_{n} \in \mathfrak{X}(U)$ tais que $\mathcal{V}_{q}=\left[L_{1 q}, \ldots, L_{n q}\right]$ para cada $q \in U$. Então existem $c_{j k}^{\nu} \in C^{\infty}(U), j, k, \nu=1, \ldots, n$, tais que

$$
\left[L_{j}, L_{k}\right]=\sum_{\nu=1}^{n} c_{j k}^{\nu} L_{\nu}, j, k=1, \ldots, n
$$


Demonstração. De fato, como $L_{1}, \ldots, L_{n} \in \mathfrak{X}(U)$ existem $a_{j k} \in C^{\infty}(U), j=1, \ldots, n, k=$ $1, \ldots, N$, tais que

$$
L_{j}=\sum_{k=1}^{N} a_{j k} \frac{\partial}{\partial x_{k}} .
$$

Além disso, para cada $q \in U,\left\{L_{1 q}, \ldots, L_{n q}\right\}$ é linearmente independente então a matriz

$$
\left(\begin{array}{cccc}
a_{11}(q) & a_{21}(q) & \cdots & a_{n 1}(q) \\
a_{12}(q) & a_{22}(q) & \cdots & a_{n 2}(q) \\
\vdots & \vdots & \ddots & \vdots \\
a_{1 N}(q) & a_{2 N}(q) & \cdots & a_{n N}(q)
\end{array}\right)
$$

tem posto $n$.

Rearranjando os índices tem-se que a matriz

$$
\left(\begin{array}{cccc}
a_{11}(p) & a_{21}(p) & \cdots & a_{n 1}(p) \\
a_{12}(p) & a_{22}(p) & \cdots & a_{n 2}(p) \\
\vdots & \vdots & \ddots & \vdots \\
a_{1 n}(p) & a_{2 n}(p) & \cdots & a_{n n}(p)
\end{array}\right)
$$

é invertível e, diminuindo a vizinhança $U$ se necessário, obtemos que a matriz acima é invertível para cada $q \in U$. Isso é possível pelo fato das funções $a_{j k}, j, k=1, \ldots, n$, e det serem suaves.

Considere $A: U \rightarrow \mathbb{C}^{n^{2}}$, onde $A(q)$ é dado pela matriz

$$
\left(\begin{array}{cccc}
a_{11}(q) & a_{21}(q) & \cdots & a_{n 1}(q) \\
a_{12}(q) & a_{22}(q) & \cdots & a_{n 2}(q) \\
\vdots & \vdots & \ddots & \vdots \\
a_{1 n}(q) & a_{2 n}(q) & \cdots & a_{n n}(q)
\end{array}\right)
$$

e $B: U \rightarrow \mathbb{C}^{(N-n) n}$, em que $B(q)$ é dado pela matriz

$$
\left(\begin{array}{cccc}
a_{1 n+1}(q) & a_{2 n+1}(q) & \cdots & a_{n n+1}(q) \\
a_{1 n+2}(q) & a_{2 n+2}(q) & \cdots & a_{n n+2}(q) \\
\vdots & \vdots & \ddots & \vdots \\
a_{1 N}(q) & a_{2 N}(q) & \cdots & a_{n N}(q)
\end{array}\right)
$$

Em particular, tem-se que $A$ e $B$ são funções suaves.

Afirmamos que $S_{q} \doteq\left\{L_{1 q}, \ldots, L_{n q},\left(\partial / \partial x_{n+1}\right)_{q}, \ldots,\left(\partial / \partial x_{N}\right)_{q}\right\}$ é uma base para $\mathbb{C} T_{q} \Omega$, para todo $q \in U$. De fato, note que

$$
\left(\begin{array}{cc}
A(q) & 0 \\
B(q) & I d
\end{array}\right)
$$


onde $I d$ é a matriz identidade de ordem $N-n$, é a matriz dos coeficientes dos vetores $L_{1 q}, \ldots, L_{n q},\left(\partial / \partial x_{n+1}\right)_{q}, \ldots,\left(\partial / \partial x_{N}\right)_{q}$ na base canônica de $\mathfrak{X}(U)$, como esta matriz tem determinante igual ao determinante $\operatorname{de} A(q)$ e $\operatorname{det}(A(q)) \neq 0$, concluímos que $S_{q}$ é base do espaço tangente a $\Omega$ em $q$.

Observação 3.3.5. Em particular, também concluímos que

$$
\left\{L_{1}, \ldots, L_{n},\left(\partial / \partial x_{n+1}\right), \ldots,\left(\partial / \partial x_{N}\right)\right\}
$$

é uma base para o $C^{\infty}(U)-$ módulo $\mathfrak{X}(U)$.

Por fim, visto que $\left[L_{j}, L_{k}\right] \in \mathfrak{X}(U), j, k=1, \ldots, n$ existem $c_{j k}^{\nu} \in C^{\infty}(U), j, k=$ $1, \ldots, n, \nu=1, \ldots, N$ tais que

$$
\left[L_{j}, L_{k}\right]=\sum_{\nu=1}^{n} c_{j k}^{\nu} L_{\nu}+\sum_{\nu=n+1}^{N} c_{j k}^{\nu} \frac{\partial}{\partial x_{\nu}}
$$

e $\left[L_{j}, L_{k}\right]_{q} \in \mathcal{V}_{q}$, para cada $q \in U$, então

$$
\sum_{\nu=n+1}^{N} c_{j k}^{\nu}(q)\left(\frac{\partial}{\partial x_{\nu}}\right)_{q}=0, q \in U
$$

e assim $c_{j k}^{\nu}(q)=0, q \in U, j, k=1, \ldots, n, \nu=n+1, \ldots, N$, concluindo a demonstração. 


\section{Formas Diferenciais}

Neste capítulo apresentamos os conceitos de formas diferenciais,fibrado cotangente complexificado $\mathbb{C} T^{*} \Omega$ e subfibrado vetorial complexo $\mathcal{W} \subset \mathbb{C} T^{*} \Omega$ que são essenciais na definição de estruturas localmente integráveis. Dessa forma, estas noções são bem relevantes no nosso contexto, visto que o Teorema de Baouendi-Treves é válido quando estamos trabalhando com estruturas localmente integráveis.

Definição 4.0.6. Denotaremos por $\mathfrak{N}(\Omega)$ o conjunto dual do $C^{\infty}(\Omega)$-módulo $\mathfrak{X}(\Omega)$. Chamaremos seus elementos, $\omega \in \mathfrak{N}(\Omega)$, de formas diferenciais de grau 1 (ou 1-formas). Assim

$$
\omega: \mathfrak{X}(\Omega) \rightarrow C^{\infty}(\Omega)
$$

é uma aplicação $C^{\infty}(\Omega)$-linear.

Observação 4.0.7. Sejam $\omega \in \mathfrak{N}(\Omega)$ e $L \in \mathfrak{X}(\Omega)$ tal que $L$ se anula no aberto não vazio $V \subset \Omega$, ou seja, $L_{V}=0$ então $\omega(L)$ se anula em $V$.

Com efeito, como $L_{V}=0$ tem-se que $L_{V} f=0$ para toda $f \in C^{\infty}(V)$ e então $\left.(L f)\right|_{V}=L_{V}\left(\left.f\right|_{V}\right)=0$, para toda $f \in C^{\infty}(\Omega)$. Seja $p \in V$ e mostremos que $\omega(L)(p)=0$.

Considere $(U, x)$ um sistema de coordenadas locais e $g \in C^{\infty}(\Omega)$, tais que $p \in U \subset$ $V, g(p)=1$ e $\left.g\right|_{\Omega-U}=0$.

Assim se $q \in V$

$$
(1-g(q)) L f(q)=(1-g(q)) 0=0=L f(q)
$$

e se $q \notin V$

$$
(1-g(q)) L f(q)=(1-0) L f(q)=L f(q),
$$

ou seja, $(1-g) L f=L f$.

$\log O$

$$
\omega(L)(p)=\omega((1-g) L)(p)=(1-g(p)) \omega(L)(p)=0,
$$

como $p \in V$ é arbitrário concluímos que $\left.\omega(L)\right|_{V}=0$.

Observação 4.0.8. Sejam $V \subset \Omega$ um aberto não vazio e $L \in \mathfrak{X}(V)$, então para cada $p \in V$ existe $\hat{L} \in \mathfrak{X}(\Omega)$ tal que $\hat{L}$ coincide com $L$ numa vizinhança de $p$.

Com efeito, considere $(U, x)$ um sistema de coordenadas locais e $g \in C^{\infty}(\Omega)$, tais que $p \in U, g(p)=1$ e $\left.g\right|_{\Omega-U}=0$. Defina

$$
\hat{L}(f)(q)= \begin{cases}g(q) L\left(\left.f\right|_{V}\right)(q), & q \in U \\ 0, & q \notin x^{-1}(\operatorname{supp}(\phi)) .\end{cases}
$$

Assim $\hat{L} \in \mathfrak{X}(\Omega)$ e $L_{U}=\hat{L}_{U}$. 
As observações acima nos permitem definir a restrição de uma 1 -forma $\omega \in \mathfrak{N}(\Omega)$ a um aberto $W \subset \Omega$ não vazio, ou seja, existe $\omega_{W} \in \mathfrak{N}(W)$ que torna o seguinte diagrama comutativo

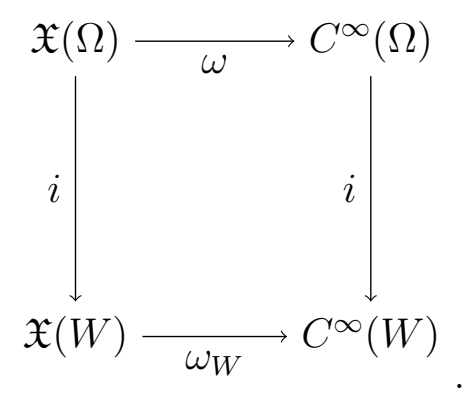

Para tanto, basta para $\omega \in \mathfrak{N}(\Omega)$ e $W \subset \Omega$ aberto não vazio, definirmos

$$
\omega_{W}(L)(p) \doteq(\omega(\tilde{L}))(p), L \in \mathfrak{X}(W), p \in W,
$$

onde $\tilde{L} \in \mathfrak{X}(\Omega)$ é tal que $L$ coincide com $\tilde{L}$ numa vizinhança de $p$.

Lema 4.0.9. Sejam $\omega \in \mathfrak{N}(\Omega), L \in \mathfrak{X}(\Omega)$ e suponha que $L_{p}=0$, para algum $p \in \Omega$. Então $\omega(L)(p)=0$.

Demonstração. Seja $(U, x)$ um sistema de coordenadas locais com $p \in U$. Observe que $L_{U} \in \mathfrak{X}(U) \mathrm{e}$

$$
L_{U}=\sum_{j=1}^{N} L_{U}\left(x_{j}\right) \frac{\partial}{\partial x_{j}}
$$

então

$$
\begin{aligned}
\omega(L)(p) & =\omega_{U}\left(L_{U}\right)(p)=\sum_{j=1}^{N} L_{U}\left(x_{j}\right)(p) \omega_{U}\left(\frac{\partial}{\partial x_{j}}\right)(p) \\
& =\sum_{j=1}^{N} L_{p}\left(\underline{x_{j}}\right) \omega_{U}\left(\frac{\partial}{\partial x_{j}}\right)(p)=0 .
\end{aligned}
$$

\subsection{Fibrado Cotangente Complexificado $\mathbb{C} T^{*} \Omega$}

Definição 4.1.1. O conjunto $\mathbb{C} T_{p}^{*} \Omega$ dual do espaço vetorial $\mathbb{C} T_{p} \Omega$, é definido como sendo o espaço cotangente à $\Omega$ em p. Por sua vez, o conjunto $\mathbb{C} T^{*} \Omega \doteq \cup_{p \in \Omega} \mathbb{C} T_{p}^{*} \Omega$ é chamado de fibrado cotangente complexificado.

Note que se $\omega \in \mathfrak{N}(\Omega), p \in \Omega$ e definirmos $\omega_{p}: \mathbb{C} T_{p} \Omega \rightarrow \mathbb{C}$ por

$$
\omega_{p}(v)=\omega(L)(p), v \in \mathbb{C} T_{p} \Omega,
$$

onde $L \in \mathfrak{X}(\Omega)$ é tal que $L_{p}=v$, então $\omega_{p} \in \mathbb{C} T_{p}^{*} \Omega$. 
Seja $(U, x)$ um sistema de coordenadas local em $\Omega$ e, para cada $j=1, \ldots, N$, defina a aplicação $C^{\infty}(U)$-linear $d x_{j}: \mathfrak{X}(U) \rightarrow C^{\infty}(U)$ tal que

$$
d x_{j}\left(\frac{\partial}{\partial x_{k}}\right)=\delta_{j k}, k=1, \ldots, N
$$

Assim $d x_{j} \in \mathfrak{N}(U)$ para $j=1, \ldots, N$, tem-se também que $\left\{d x_{1}, \ldots, d x_{N}\right\}$ é uma base para o $C^{\infty}(U)$-módulo $\mathfrak{N}(U)$ de forma que:

$$
\omega=\sum_{j=1}^{N} \omega\left(\frac{\partial}{\partial x_{j}}\right) d x_{j} .
$$

Com efeito, seja $\omega \in \mathfrak{N}(U)$ e observe que para $L \in \mathfrak{X}(U)$ tem-se que

$$
\begin{aligned}
L & =\sum_{k=1}^{N} L\left(x_{k}\right) \frac{\partial}{\partial x_{k}}, a_{k} \in C^{\infty}(U), k=1, \ldots, N, \\
d x_{j}(L) & =\sum_{k=1}^{N} a_{k} d x_{j}\left(\frac{\partial}{\partial x_{k}}\right)=a_{j}, j=1, \ldots, N,
\end{aligned}
$$

então

$$
\omega(L)=\sum_{k=1}^{N} d x_{k}(L) \omega\left(\frac{\partial}{\partial x_{k}}\right)=\left(\sum_{k=1}^{N} \omega\left(\frac{\partial}{\partial x_{k}}\right) d x_{k}\right)(L) .
$$

Analogamente obtemos que $\left\{d x_{1 p}, \ldots, d x_{N p}\right\} \subset \mathbb{C} T_{p}^{*} \Omega$ é a base dual de $\left\{\partial / \partial x_{1 p}, \ldots, \partial / \partial x_{N p}\right\}$.

Proposição 4.1.2. O espaço cotangente à $\Omega$ em p pode ser descrito da seguinte forma:

$$
\mathbb{C} T_{p}^{*} \Omega=\left\{\omega_{p} ; \omega \in \mathfrak{N}(\Omega)\right\}
$$

Demonstração. Considere $\eta \in \mathbb{C} T_{p}^{*} \Omega$, então existem $\eta_{1}, \ldots, \eta_{N} \in \mathbb{C}$ tais que

$$
\eta=\sum_{j=1}^{N} \eta_{j}\left(d x_{j}\right)_{p}
$$

Sejam $(U, x)$ um sistema de coordenadas locais e $g \in C^{\infty}(\Omega)$, tais que $p \in U, g(p)=1$ e $\left.g\right|_{\Omega-U}=0$.

Defina $\omega: \mathfrak{X}(\Omega) \rightarrow C^{\infty}(\Omega)$, dada por:

$$
\omega(L)(q)= \begin{cases}g(q) \sum_{j=1}^{N} \eta_{j} d x_{j}(L)(q), & q \in U \\ 0, & q \notin x^{-1}(\operatorname{supp}(\phi)),\end{cases}
$$

para $L \in \mathfrak{X}(\Omega)$ e $q \in \Omega$.

Se $L, M \in \mathfrak{X}(\Omega)$ e $f \in C^{\infty}(\Omega)$, tem-se:

$$
\omega(L+f M)(q)= \begin{cases}g(q)\left(\sum_{j=1}^{N} \eta_{j} d x_{j}(L)(q)+\eta_{j} f(q) d x_{j}(M)(q)\right), & q \in U \\ 0, & q \notin x^{-1}(\operatorname{supp}(\phi)),\end{cases}
$$


ou seja, $\omega(L+f M)=\omega(L)+f \omega(M)$ e $\operatorname{assim} \omega \in \mathfrak{N}(\Omega)$.

Além disso, se $v \in \mathbb{C} T_{p} \Omega$ tem-se

$$
\begin{aligned}
\omega_{p}(v) & =\omega_{p}\left(\sum_{j=1}^{N} v_{j}\left(\frac{\partial}{\partial x_{j}}\right)_{p}\right)=g(p) \sum_{k=1}^{N} \eta_{k} d x_{k p}\left(\sum_{j=1}^{N} v_{j}\left(\frac{\partial}{\partial x_{j}}\right)_{p}\right)= \\
& =\sum_{k=1}^{N} \eta_{k} d x_{k p}(v)=\eta(v)
\end{aligned}
$$

$\log \mathrm{O} \omega_{p}=\eta$

Definição 4.1.3. Dada $f \in C^{\infty}(\Omega)$, definimos $d f \in \mathfrak{N}(\Omega)$ da seguinte maneira

$$
d f(L) \doteq L(f), L \in \mathfrak{X}(\Omega) .
$$

Observe que de 4.1 tem-se que

$$
d f=\sum_{j=1}^{N} d f\left(\frac{\partial}{\partial x_{j}}\right) d x_{j}=\sum_{j=1}^{N} \frac{\partial f}{\partial x_{j}} d x_{j} .
$$

\subsection{Subfibrado Vetorial Complexo $\mathcal{W} \subset \mathbb{C} T^{*} \Omega$}

Definição 4.2.1. Dizemos que o conjunto $\mathcal{W} \doteq \cup_{p \in \Omega} \mathcal{W}_{p} \subset \mathbb{C} T^{*} \Omega$ é um subfibrado vetorial complexo $m$-dimensional se satisfaz as seguintes propriedades:

1. para cada $p \in \Omega, \mathcal{W}_{p} \subset \mathbb{C} T_{p}^{*} \Omega$ é um subespaço vetorial $m$-dimensional;

2. para cada $p_{0} \in \Omega$, existem $U \subset \Omega$ aberto com $p_{0} \in U$ e $\omega_{1}, \ldots, \omega_{m} \in \mathfrak{N}(U)$ tais que, para cada $p \in U$, vale que $\mathcal{W}_{p}=\left[\omega_{1 p}, \ldots, \omega_{m p}\right]$.

Proposição 4.2.2. Seja $\mathcal{V}=\cup_{p \in \Omega} \mathcal{V}_{p}$ um subfibrado vetorial complexo $n$-dimensional de $\mathbb{C} T \Omega$ e defina, para cada $p \in \Omega$, o seguinte conjunto

$$
\mathcal{V}_{p}^{\perp} \doteq\left\{\eta \in \mathbb{C} T_{p}^{*} \Omega ; \eta(v)=0, \forall v \in \mathcal{V}_{p}\right\}
$$

Então $\mathcal{V}^{\perp}=\cup_{p \in \Omega} \mathcal{V}_{p}^{\perp}$ é um subfibrado vetorial complexo $m$-dimensional de $\mathbb{C} T^{*} \Omega$, onde $m=N-n$.

Demonstração. Inicialmente, vamos mostrar que, para cada $p \in \Omega, \mathcal{V}_{p}^{\perp} \subset \mathbb{C} T_{p}^{*} \Omega$ é subespaço vetorial $m$-dimensional. Sejam $\eta_{1}, \eta_{2} \in \mathcal{V}_{p}^{\perp}$ e $\lambda \in \mathbb{C}$, então

$$
\left(\eta_{1}+\lambda \eta_{2}\right)(v)=\eta_{1}(v)+\lambda \eta_{2}(v)=0, \forall v \in \mathcal{V}_{p}
$$

segue que $\eta_{1}+\lambda \eta_{2} \in \mathcal{V}_{p}^{\perp}$ e $\mathcal{V}_{p}^{\perp}$ é subespaço vetorial do espaço cotangente a $\Omega$ em $p$. 
Seja $\left\{v_{1}, \ldots, v_{n}\right\}$ uma base para $\mathcal{V}_{p}$, então existem $v_{n+1}, \ldots, v_{N} \in \mathbb{C} T_{p} \Omega$ tal que $\left\{v_{1}, \ldots, v_{N}\right\}$ é uma base para o espaço tangente a $\Omega$ em $p$. Considere $\left\{\eta_{1}, \ldots, \eta_{N}\right\}$ base dual de $\left\{v_{1}, \ldots, v_{N}\right\}$.

Além disso, se $\eta \in \mathcal{V}_{p}^{\perp}$ então $\eta\left(v_{j}\right)=0, j=1, \ldots, n$ e

$$
\eta=\sum_{j=1}^{N} \alpha_{j} \eta_{j}
$$

onde $\alpha_{j}=\eta\left(v_{j}\right), j=1, \ldots, N$. Logo $\eta=\sum_{j=n+1}^{N} \alpha_{j} \eta_{j}$, e portanto $\mathcal{V}_{p}^{\perp}=\left[\eta_{n+1 p}, \ldots, \eta_{N p}\right]$ e $\mathcal{V}_{p}^{\perp}$ é um espaço vetorial $m$-dimensional.

Dado $p_{0} \in \Omega$, sejam $\left(U_{0}, x\right)$ sistema de coordenadas locais com $p_{0} \in U_{0}$ e $L_{1}, \ldots, L_{n}$ $\in \mathfrak{X}\left(U_{0}\right)$ tais que $\mathcal{V}_{p}=\left[L_{1 p}, \ldots, L_{n p}\right]$, para todo $p \in U_{0}$.

Assim para $j=1, \ldots, n$ e $k=1, \ldots, N$, existem $a_{j k} \in C^{\infty}\left(U_{0}\right)$ tais que

$$
L_{j}=\sum_{k=1}^{N} a_{j k} \frac{\partial}{\partial x_{k}} .
$$

Como, para cada $p \in U_{0},\left\{L_{j p}\right\}_{j=1}^{n}$ é LI, então a matriz

$$
\left(\begin{array}{cccc}
a_{11}(p) & a_{21}(p) & \cdots & a_{n 1}(p) \\
a_{12}(p) & a_{22}(p) & \cdots & a_{n 2}(p) \\
\vdots & \vdots & \ddots & \vdots \\
a_{1 N}(p) & a_{2 N}(p) & \cdots & a_{n N}(p)
\end{array}\right)
$$

tem posto $n$.

Logo podemos rearranjar os índices $j, k$ de forma que a matriz

$$
\left(\begin{array}{cccc}
a_{11}\left(p_{0}\right) & a_{21}\left(p_{0}\right) & \cdots & a_{n 1}\left(p_{0}\right) \\
a_{12}\left(p_{0}\right) & a_{22}\left(p_{0}\right) & \cdots & a_{n 2}\left(p_{0}\right) \\
\vdots & \vdots & \ddots & \vdots \\
a_{1 n}\left(p_{0}\right) & a_{2 n}\left(p_{0}\right) & \cdots & a_{n n}\left(p_{0}\right)
\end{array}\right)
$$

possui determinante não nulo. Além disso, como o as funções det e $a_{j k}, j=1, \ldots, n, k=$ $1, \ldots, N$ são suaves então, diminuindo a vizinhança $U_{0}$ se necessário, concluímos que a matriz acima é invertível para cada $p \in U_{0}$.

Sejam $b_{j k} \in C^{\infty}\left(U_{0}\right), j, k=1, \ldots, n$ tais que

$$
\sum_{j=1}^{n} a_{l j}(p) b_{j k}(p)=\sum_{j=1}^{n} b_{l j}(p) a_{j k}(p)=\delta_{l k}, p \in U_{0}, l, k=1, \ldots, n .
$$

Defina

$$
L_{j}^{\#}=\sum_{\nu=1}^{n} b_{j \nu} L_{\nu}, j=1, \ldots, n
$$


Como, para cada $p \in U_{0}$, a matriz formada pelos coeficientes $b_{j \nu}(p)$ é invertível então tal matriz é uma matriz de mudança de base, e portanto $\left\{L_{j p}^{\#}\right\}_{j=1}^{n}$ é uma base para $\mathcal{V}_{p}$.

Observe que

$$
\begin{aligned}
L_{j}^{\#} & =\sum_{\nu=1}^{n} b_{j \nu} L_{\nu}=\sum_{\nu=1}^{n} \sum_{k=1}^{N} b_{j \nu} a_{\nu k} \frac{\partial}{\partial x_{k}} \\
& =\frac{\partial}{\partial x_{j}}+\sum_{k=n+1}^{N}\left(\sum_{\nu=1}^{n} b_{j \nu} a_{\nu k}\right) \frac{\partial}{\partial x_{k}} .
\end{aligned}
$$

Defina

$$
c_{j k} \doteq \sum_{\nu=1}^{n} b_{j \nu} a_{\nu k+n}, j=1, \ldots, n, k=1, \ldots, N-m
$$

então

$$
L_{j}^{\#}=\frac{\partial}{\partial x_{j}}+\sum_{k=1}^{m} c_{j k} \frac{\partial}{\partial x_{n+k}}
$$

Agora tome

$$
\omega_{l} \doteq d x_{n+l}-\sum_{\gamma=1}^{n} c_{\gamma l} d x_{\gamma} \in \mathfrak{N}\left(U_{0}\right), l=1, \ldots, m .
$$

Vamos mostrar, para cada $p \in U_{0}$, que $\mathcal{V}_{p}^{\perp}=\left[\omega_{1 p}, \ldots, \omega_{m p}\right]$. Para tanto basta mostrar que $\left\{\omega_{l p}\right\}_{l=1}^{m} \subset \mathcal{V}_{p}^{\perp}$ e que este conjunto é linearmente independente.

Note que

$$
\begin{aligned}
\omega_{l p}\left(L_{j p}^{\#}\right) & =d x_{n+l}\left(\frac{\partial}{\partial x_{k}}\right)(p)+\sum_{j=n+1}^{N} c_{k j}(p) d x_{n+l}\left(\frac{\partial}{\partial x_{j}}\right)(p)- \\
& -\sum_{\gamma=1}^{n} c_{\gamma l}(p) d x_{\gamma}\left(\frac{\partial}{\partial x_{k}}\right)(p)-\sum_{\gamma=1}^{n} \sum_{k=1}^{m} c_{\gamma l}(p) c_{j k}(p) d x_{\gamma}\left(\frac{\partial}{\partial x_{k}}\right)(p) \\
& =-c_{j l}(p)+c_{j l}(p)=0, l=1, \ldots, m, j=1, \ldots, n .
\end{aligned}
$$

Como $\mathcal{V}_{p}=\left[L_{1 p}^{\#}, \ldots, L_{n p}^{\#}\right]$, tem-se que $\left\{\omega_{l p}\right\}_{l=1}^{m} \subset \mathcal{V}_{p}^{\perp}$. Além disso, se $\alpha_{l} \in \mathbb{C}, l=$ $1, \ldots, m$ forem tais que

consequentemente

$$
\sum_{l=1}^{m} \alpha_{l} \omega_{l p}=0
$$

$$
\sum_{l=1}^{m} \alpha_{l} \omega_{l}\left(\frac{\partial}{\partial x_{n+j}}\right)(p)=\alpha_{j}, j=1, \ldots, m,
$$

então $\alpha_{j}=0, j=1, \ldots, m$ e concluímos que $\left\{\omega_{l p}\right\}_{l=1}^{m}$ é linearmente independente completando a demonstração.

Observação 4.2.3. É importante ressaltar também que se $\mathcal{V}^{\perp}$ for um subfibrado vetorial complexo $m$-dimensional de $\mathbb{C} T^{*} \Omega$, então $\mathcal{V}$ é um subfibrado vetorial complexo $n$-dimensional de $\mathbb{C} T \Omega$ com $n=N-m$. Além disso, quando $\mathcal{V}$ é uma estrutura involutiva, denotamos $\mathcal{V}^{\perp}$ simplesmente por $T^{\prime}$. 


\subsection{Conjugação Complexa}

Definição 4.3.1 (Conjugação Complexa). Dado $L \in \mathfrak{X}(\Omega)$ definimos seu campo vetorial conjugado $\bar{L} \in \mathfrak{X}(\Omega)$ da seguinte forma

$$
\bar{L}(f) \doteq \overline{L(\bar{f})}, f \in C^{\infty}(\Omega) .
$$

Além disso, dizemos que um campo $L \in \mathfrak{X}(\Omega)$ é real se $L=\bar{L}$.

Se $\omega \in \mathfrak{N}(\Omega)$, definimos sua forma diferencial conjugada $\bar{\omega} \in \mathfrak{N}(\Omega)$ por $\bar{\omega}(L) \doteq$ $\overline{\omega(\bar{L})}$ e dizemos que tal forma é real se $\omega=\bar{\omega}$.

Se $v \in \mathbb{C} T_{p} \Omega$, definimos seu conjugado $\bar{v} \in \mathbb{C} T_{p} \Omega$ por $\bar{v}(\underline{f}) \doteq \overline{v(\bar{f})}$ e dizemos que tal forma é real se $v=\bar{v}$. Denotamos por $T_{p} \Omega$ o conjunto formado por todos os vetores tangentes à $\Omega$ em p que são reais.

Se $\eta \in \mathbb{C} T_{p}^{*} \Omega$, definimos seu conjugado $\bar{\eta} \in \mathbb{C} T_{p}^{*} \Omega$ por $\bar{\eta}(v) \doteq \overline{\eta(\bar{v})}$ e dizemos que tal forma é real se $\eta=\bar{\eta}$. Denotamos por $T_{p}^{*} \Omega$ o conjunto formado por todos os vetores cotangentes à $\Omega$ em p que são reais.

Observação 4.3.2. Dado um campo vetorial $L \in \mathfrak{X}(\Omega)$ é fácil notar que $L$ é real se, $e$ somente se, $L\left(C^{\infty}(\Omega ; \mathbb{R})\right) \subset C^{\infty}(\Omega ; \mathbb{R})$.

Além disso, se $(U, x)$ é um sistema de coordenadas locais com $p \in U$, então valem as seguintes afirmações:

1. $L \in \mathfrak{X}(U)$ é real se, e somente se, $L=\sum_{j=1}^{N} a_{j} \frac{\partial}{\partial x_{j}}$ com $a_{j} \in C^{\infty}(\Omega ; \mathbb{R}), j=1, \ldots, N$;

2. $\omega \in \mathfrak{N}(U)$ é real se, e somente se, $\omega=\sum_{j=1}^{N} a_{j} d x_{j}$ com $a_{j} \in C^{\infty}(\Omega ; \mathbb{R}), j=1, \ldots, N$;

3. $v \in \mathbb{C} T_{p} \Omega$ é real se, e somente se, $v=\sum_{j=1}^{N} v_{j} \frac{\partial}{\partial x_{j}} p$ com $v_{j} \in \mathbb{R}, j=1, \ldots, N$;

4. $\eta \in \mathbb{C} T_{p}^{*} \Omega$ é real se, e somente se, $\eta=\sum_{j=1}^{N} \eta_{j} d x_{j p}$ com $\eta_{j} \in \mathbb{R}, j=1, \ldots, N$.

Observação 4.3.3. Se $\mathcal{V}=\cup_{p \in \Omega} \mathcal{V}_{p}$ é subfibrado vetorial complexo $n$-dimensional de $\mathbb{C} T \Omega$, então $\overline{\mathcal{V}}=\cup_{p \in \Omega} \overline{\mathcal{V}_{p}}$ é, por sua vez, um subfibrado vetorial complexo $n$-dimensional de $\mathbb{C} T \Omega$, onde

$$
\overline{\mathcal{V}_{p}} \doteq\left\{\bar{v} \in \mathbb{C} T_{p} \Omega ; v \in \mathcal{V}_{p}\right\}
$$

Além disso, tem-se que $\overline{\mathcal{V}}^{\perp}=\overline{\mathcal{V}^{\perp}}$. 



\section{Teorema de Frobenius}

\subsection{Problema de Cauchy}

Seja $U \subset \mathbb{R}^{N}$ um aberto que contém a origem e considere $L \in \mathfrak{X}(U)$ um campo vetorial real. Assim

$$
L=\sum_{j=1}^{N} a_{j} \frac{\partial}{\partial x_{j}}
$$

onde $x(p)=i d(p)=p, p \in U$. Assim $a_{j} \in C^{\infty}(U ; \mathbb{R}), j=1, \ldots, N$.

Suponha que $L_{0} \neq 0$, então, para algum $j_{0} \in\{1, \ldots, N\}, a_{j_{0}}(0) \neq 0$. Logo podemos supor, sem perda de generalidade, que $a_{1}(0) \neq 0$.

Vamos encontrar coordenadas locais $(W, y)$, onde $W \subset \mathbb{R}^{N}$ é um aberto que contém a origem e $y=\left(y_{1}, \ldots, y_{N}\right)$ é tal que

$$
L=\frac{\partial}{\partial y_{1}}
$$

Em outras palavras, procuramos um aberto que contém a origem $W \subset \mathbb{R}^{N}$ e $\phi: W \rightarrow U$ um difeomorfismo dado por $\phi(q)=p, q \in W \operatorname{com} \phi(0)=0$ e tal que para cada $f \in C^{\infty}(U)$ vale:

$$
\begin{aligned}
\frac{\partial f}{\partial y_{1}}(p) & =\frac{\partial(f \circ \phi)}{\partial e_{1}}\left(\phi^{-1}(p)\right)=(D f)(p) D \phi\left(\phi^{-1}(p)\right) e_{1}= \\
& =\sum_{j=1}^{N} \frac{\partial f}{\partial x_{j}}(p) \frac{\partial \phi_{j}}{\partial e_{1}}\left(\phi^{-1}(p)\right)=\sum_{j=1}^{N} a_{j}(p) \frac{\partial f}{\partial x_{j}}(p)=L(f)(p), p \in U
\end{aligned}
$$

em que

$$
\frac{\partial \phi}{\partial e_{k}}(q)=\lim _{h \rightarrow 0} \frac{\phi(q+h)-\phi(q)}{h}, k=1, \ldots, N
$$

Tal aplicação $\phi$ satisfaz

$$
\begin{aligned}
\frac{\partial \phi_{j}}{\partial e_{1}}(q) & =a_{j}(\phi(q)), q \in U, j=1, \ldots, N \\
\phi_{j}\left(0, q_{2}, \ldots, q_{N}\right) & =q_{j}, j=1, \ldots, N \\
\phi_{1}\left(0, q_{2}, \ldots, q_{N}\right) & =0,
\end{aligned}
$$

assim tem-se que

$$
\frac{\partial \phi_{j}}{\partial e_{k}}\left(0, q_{2}, \ldots, q_{N}\right)=\delta_{j k}, j, k=2, \ldots, N,
$$

sendo que $\delta_{j k}$ é o delta de Kronecker e então

$$
\begin{aligned}
& \frac{\partial \phi_{j}}{\partial e_{k}}(0, \ldots, 0)=\delta_{j k}, j, k=2, \ldots, N \\
& \frac{\partial \phi_{j}}{\partial e_{1}}(0, \ldots, 0)=a_{j}(\phi(0))=a_{j}(0), j=1, \ldots, N .
\end{aligned}
$$


Logo det $D \phi(0)=a_{1}(0) \neq 0$ e, pelo Teorema da Função Inversa, obtemos que $\phi$ é um difeomorfismo em torno da origem. Tomando $y=\left(y_{1}, \ldots, y_{N}\right) \doteq \phi^{-1}$ obtemos um sistema de coordenadas local $(W, y)$ de forma que $L=\frac{\partial}{\partial y_{1}}$.

Vamos mostrar agora que existe uma função que satisfaz as propriedades da função $\phi$ descrita acima. Para tanto, primeiramente note que como $0 \in U$, existe $\delta>0$ tal que $\overline{B(0, \delta)} \subset U$.

Seja $\varepsilon>0$ e considere o conjunto

$$
E_{\varepsilon} \doteq\left\{u: K_{\varepsilon} \rightarrow \mathbb{R}^{N} ;\|u\|_{\infty} \leq \delta, u \in C\left(K_{\varepsilon}\right)\right\}
$$

onde $K_{\varepsilon} \doteq \overline{B(0, \varepsilon)}$. Note que tal conjunto, equipado com a norma $\|\cdot\|_{\infty}$, é um espaço métrico completo não vazio.

Defina

$$
\begin{aligned}
T: E_{\varepsilon} & \rightarrow C\left(K_{\varepsilon}\right) \\
u & \mapsto T u,
\end{aligned}
$$

em que

$$
T u\left(t_{1}, \ldots, t_{N}\right)=\left(0, t_{2}, \ldots, t_{N}\right)+\int_{0}^{t_{1}} a\left(u\left(t, t_{2}, \ldots, t_{N}\right)\right) d t .
$$

Esta aplicação é contínua. Note também que como cada $a_{j}$ é infinitamente diferenciável, existe $M_{1} \doteq \max _{\left(t_{1}, \ldots, t_{N}\right) \in \overline{B(0, \delta)}}\left\|a\left(t_{1}, \ldots, t_{N}\right)\right\|$ e assim:

$$
\|T u(t)\| \leq\|t\|+M_{1} t_{1} \leq \varepsilon\left(1+M_{1}\right) .
$$

Daí, se $0<\varepsilon<\frac{\delta}{1+M_{1}}$ tem-se que a imagem de $T u$ é um subconjunto de $\overline{B(0, \delta)}$ para qualquer $u \in E_{\varepsilon}$, ou seja,

$$
\begin{aligned}
T: E_{\varepsilon} & \rightarrow E_{\varepsilon} \\
u & \mapsto T u
\end{aligned}
$$

Note também que

$$
\left\|T u_{1}-T u_{2}\right\|_{\infty}=\sup _{t \in K_{\varepsilon}}\left|T u_{1}(t)-T u_{2}(t)\right|
$$

mas

$$
\begin{aligned}
\left|T u_{1}(s)-T u_{2}(s)\right| & \leq \int_{0}^{t_{1}}\left|a\left(u_{1}\left(s, t_{2}, \ldots, t_{N}\right)\right)-a\left(u_{2}\left(s, t_{2}, \ldots, t_{N}\right)\right)\right| d s \leq \\
& \leq M\left|t_{1}\right|\left\|u_{1}-u_{2}\right\|_{\infty} \leq \varepsilon M\left\|u_{1}-u_{2}\right\|_{\infty},
\end{aligned}
$$

sendo que

$$
M=\sup _{t \in \overline{B(0,3 \delta)}}\|\nabla a(t)\|<\infty
$$


é tal que $|a(x)-a(y)| \leq M\|x-y\|$, para $x, y \in \overline{B(0, \delta)}$.

Por fim, tomando $0<\epsilon<\min \left\{\frac{1}{2 M}, \frac{\delta}{1+M_{1}}\right\}$, obtemos que $T: E_{\varepsilon} \rightarrow E_{\varepsilon}$ é uma contração. Portanto, pelo Teorema do Ponto Fixo de Banach, existe $\phi \in E_{\varepsilon}$ tal que $T \phi=\phi$. Assim

$$
\begin{aligned}
\phi(B(0, \varepsilon)) & \subset U, \\
\phi & \in C^{\infty}(B(0, \varepsilon)), \\
\phi(0) & =T \phi(0)=0, \\
\frac{\partial \phi}{\partial e_{1}} & =a \\
\phi\left(0, y_{2}, \ldots, y_{N}\right) & =\left(0, y_{2}, \ldots, y_{N}\right) .
\end{aligned}
$$

\subsection{Teorema de Frobenius}

Teorema 5.2.1 (Frobenius). Sejam $V \subset \mathbb{R}^{N}$ aberto que contém a origem e $L_{1}, \ldots, L_{n} \in$ $\mathfrak{X}(V)$ campos vetoriais reais tais que

$$
\left\{L_{1 p}, \ldots, L_{n p}\right\}
$$

é linearmente independente, para todo $p \in V$. Assuma que o subfibrado vetorial complexo $n$-dimensional $\mathcal{V} \subset \mathbb{C} T V$ gerado por estes vetores é uma estrutura involutiva. Então existe um sistema de coordenadas locais $(W, y), y \doteq\left(y_{1}, \ldots, y_{N}\right)$, com $0 \in W$ tal que $\mathcal{V} e ́$ gerado pelos campos $\left\{\frac{\partial}{\partial y_{1}}, \ldots, \frac{\partial}{\partial y_{n}}\right\}$.

Demonstração. Vamos utilizar o processo de indução. Para $n=1$, tem-se $L \in \mathfrak{X}(V)$ campo real tal que $L_{0} \neq 0$. Pelo que foi feito na seção anterior concluímos que existe sistema de coordenadas locais $(W, y)$, com $0 \in W, y \doteq\left(y_{1}, \ldots, y_{N}\right)$ tais que

$$
L=\frac{\partial}{\partial y_{1}} .
$$

Então $\mathcal{V}$ é gerado por $\left\{\partial / \partial y_{1}\right\}$.

Suponha, por hipótese de indução, que o resultado vale para valores menores que $n$. Visto que $\left\{L_{1 p}, \ldots, L_{n p}\right\}$ gera $\mathcal{V}_{p}, p \in V$, tem-se que $L_{10} \neq 0$ então, diminuindo a vizinhaça $V$ se necessário, existe um difeomorfismo

$$
\begin{aligned}
\xi: V & \rightarrow \xi(V) \\
p & \mapsto \xi(p),
\end{aligned}
$$

$\operatorname{com} \xi(0)=0$, tal que

$$
L_{1}=\frac{\partial}{\partial \xi_{1}}, L_{j}=\sum_{k=1}^{N} a_{j k} \frac{\partial}{\partial \xi_{k}}, j=2, \ldots, n
$$


Defina

$$
\begin{aligned}
& L_{1}^{\#} \doteq L_{1}, \\
& L_{j}^{\#} \doteq L_{j}-a_{j 1} L_{1}, j=2, \ldots, n
\end{aligned}
$$

assim

$$
L_{j}^{\#}=\sum_{k=1}^{N} a_{j k} \frac{\partial}{\partial \xi_{k}}-a_{j 1} \frac{\partial}{\partial \xi_{1}}=\sum_{k=2}^{N} a_{j k} \frac{\partial}{\partial \xi_{k}} .
$$

Note que, para cada $p \in V$, tem-se

$$
\begin{aligned}
L_{1 p}^{\#} & =L_{1 p} \in\left[L_{1 p}, \ldots, L_{n p}\right] \\
L_{j p}^{\#} & =L_{j p}-a_{j 1}(p) L_{1 p} \in\left[L_{1 p}, \ldots, L_{n p}\right], j=2, \ldots, n .
\end{aligned}
$$

Além disso, se $\alpha_{1}, \ldots, \alpha_{n} \in \mathbb{C}$ e $p \in V$ forem tais que

$$
\sum_{j=1}^{n} \alpha_{j} L_{j p}^{\#}=0
$$

consequentemente tem-se que

$$
\left(\alpha_{1}-\sum_{j=2}^{n} \alpha_{j} a_{j 1}(p)\right) L_{1 p}+\sum_{j=2}^{n} \alpha_{j} L_{j p}=0
$$

$\log \mathrm{O}$

$$
\begin{gathered}
\alpha_{j}=0, j=2, \ldots, n, \\
\alpha_{1}=\sum_{j=2}^{n} \alpha_{j} a_{j 1}(p)=0
\end{gathered}
$$

e $\left\{L_{j p}^{\#}\right\}_{j=1}^{n}$ é linearmente independente, ou seja, $\left[L_{1 p}^{\#}, \ldots, L_{n p}^{\#}\right]=\left[L_{1 p}, \ldots, L_{n p}\right]=\mathcal{V}_{p}, p \in V$.

Pela Proposição 3.3.4, existem $c_{j k}^{\nu} \in C^{\infty}(V), j, k, \nu=1, \ldots, n$, tais que

$$
\left[L_{j}^{\#}, L_{k}^{\#}\right]=\sum_{\nu=1}^{n} c_{j k}^{\nu} L_{\nu}^{\#}, j, k=1, \ldots, n
$$

Observe que

$$
\left[L_{j}^{\#}, L_{k}^{\#}\right]=\sum_{\nu=1}^{n} c_{j k}^{\nu} L_{\nu}^{\#}=c_{j k}^{1} \frac{\partial}{\partial \xi_{1}}+\sum_{\nu=2}^{n} c_{j k}^{\nu} L_{\nu}^{\#}, j, k=2, \ldots, n
$$

então

$$
c_{j k}^{1}(p)=\left[L_{j}^{\#}, L_{k}^{\#}\right]_{p}\left(d \xi_{1 p}\right), p \in V
$$

por outro lado

$$
\left[L_{j}^{\#}, L_{k}^{\#}\right]_{p}\left(d \xi_{1 p}\right)=L_{j}^{\#}\left(\sum_{\nu=2}^{n} a_{k \nu}(p) \frac{\partial \xi_{1}}{\partial \xi_{\nu}}(p)\right)-L_{\nu}^{\#}\left(\sum_{\nu=2}^{n} a_{j \nu}(p) \frac{\partial \xi_{1}}{\partial \xi_{\nu}}(p)\right)=0, j, k \geq 2
$$


$\operatorname{assim} c_{j k}^{1}=0, j, k \geq 2 \mathrm{e}$

$$
\left[L_{j}^{\#}, L_{k}^{\#}\right]=\sum_{\nu=2}^{n} c_{j k}^{\nu} L_{\nu}^{\#}, j, k=2, \ldots, n
$$

Seja $\delta>0$ tal que $(-\delta, \delta)^{N} \subset \xi(V)$ e tome $W \doteq(-\delta, \delta)^{N-1} \subset \mathbb{R}^{N-1}$. Além disso, diminuindo a vizinhança $V$ se necessário, podemos supor $\xi(V)=(-\delta, \delta)^{N}$. Note que $W$ é uma variedade suave com estrutura diferenciável herdada de $\mathbb{R}^{N-1}$.

Dado $L \in \mathfrak{X}(V)$, vamos definir $L_{U} \in \mathfrak{X}(U)$, onde $U \doteq \xi(V) \subset \mathbb{R}^{N}$, o qual é dado por

$$
\begin{aligned}
L_{U}: C^{\infty}(U) & \rightarrow C^{\infty}(U) \\
g & \mapsto L_{U}(g),
\end{aligned}
$$

onde $L_{U}(g) \doteq L(g \circ \xi) \circ \xi^{-1}$.

Observação 5.2.2. Note que $g \circ \xi, L(g \circ \xi) \circ \xi^{-1} \in C^{\infty}(U) e$

$$
\begin{aligned}
L_{U}\left(g_{1}+\lambda g_{2}\right) & =L\left(\left(g_{1}+\lambda g_{2}\right) \circ \xi\right) \circ \xi^{-1}=\left(L\left(g_{1} \circ \xi\right)+\lambda L\left(g_{2} \circ \xi\right)\right) \circ \xi^{-1}= \\
& =L\left(g_{1} \circ \xi\right) \circ \xi^{-1}+\lambda L\left(g_{2} \circ \xi\right) \circ \xi^{-1}= \\
& =L_{U}\left(g_{1}\right)+\lambda L_{U}\left(g_{2}\right), g_{1}, g_{2} \in C^{\infty}(U), \lambda \in \mathbb{C},
\end{aligned}
$$

então $L_{U} \in \mathfrak{X}(U)$.

Agora, dado $L \in \mathfrak{X}(U)$, definiremos $\left.L\right|_{W} \in \mathfrak{X}(W)$. Primeiramente, dada $f \in$ $C^{\infty}(W)$ defina

$$
\begin{aligned}
\hat{f}: U & \rightarrow \mathbb{C} \\
(t, q) & \mapsto \hat{f}(t, q),
\end{aligned}
$$

onde $\hat{f}(t, q) \doteq f(q)$, em particular tem-se que $\hat{f} \in C^{\infty}(U)$. Definimos $\left.L\right|_{W}$ por

$$
\left.L\right|_{W}(f)(q) \doteq L(\hat{f})(0, q), f \in C^{\infty}(W), q \in W .
$$

Observação 5.2.3. Observe que $\left.L\right|_{W} \in \mathfrak{X}(W)$. Com efeito, se $f_{1}, f_{2} \in C^{\infty}(W), \lambda \in \mathbb{C}$ então $\hat{f}_{j}(t, q)=\hat{f}_{j}(q), j=1,2, e\left(\hat{f}_{1}+\lambda \hat{f}_{2}\right)(t, q)=\left(f_{1}+\lambda f_{2}\right)(q)$. Assim

$$
\begin{aligned}
\left.L\right|_{W}\left(f_{1}+\lambda f_{2}\right)(q) & =L\left(\hat{f}_{1}+\lambda \hat{f}_{2}\right)(0, q)=L\left(\hat{f}_{1}\right)(0, q)+\lambda L\left(\hat{f}_{2}\right)(0, q)= \\
& =\left(\left.L\right|_{W}\left(f_{1}\right)+\left.\lambda L\right|_{W}\left(f_{2}\right)\right)(q), q \in W .
\end{aligned}
$$

Agora, para cada $j=2, \ldots, n$, tome

$$
\left.M_{j} \doteq\left(L_{j}^{\#}\right)\right|_{W} \in \mathfrak{X}(W), j=2, \ldots, n,
$$


ou seja,

$$
M_{j}=\left.\sum_{k=2}^{N}\left(a_{j l} \circ \xi^{-1} \circ \psi\right) \frac{\partial}{\partial \xi_{l}}\right|_{W}, j=2, \ldots, n,
$$

em que

$$
\begin{aligned}
\psi: W & \rightarrow U \\
q & \mapsto(0, q) .
\end{aligned}
$$

Vamos mostrar que, para cada $q \in W,\left\{M_{j q}\right\}_{j=2}^{n}$ é linearmente independente. Para tanto precisamos fazer algumas observações.

Para cada $q_{0} \in W$, seja $p_{0} \doteq \xi^{-1}\left(0, q_{0}\right) \in V$. Para $\underline{f} \in C^{\infty}\left(p_{0}\right)$, tem-se que $f \in$ $C^{\infty}\left(V_{1}\right)$, onde $V_{1} \subset V$ é uma vizinhança de $p_{0}$. Defina

$$
\begin{aligned}
\tilde{f}: W_{1} & \rightarrow \mathbb{C} \\
q & \mapsto \tilde{f}(q) \doteq\left(f \circ \xi^{-1}\right)(0, q),
\end{aligned}
$$

onde $W_{1} \doteq(-\epsilon, \epsilon)^{N-1}$ e $\epsilon>0$ é tal que $\xi\left(p_{0}\right) \in(-\epsilon, \epsilon)^{N} \subset \xi\left(V_{1}\right)$, assim $\underline{\tilde{f}} \in C^{\infty}\left(q_{0}\right)$.

Observação 5.2.4. Afirmamos que, para $\underline{f} \in C^{\infty}\left(p_{0}\right), M_{j q_{0}}(\underline{\tilde{f}})=L_{j p_{0}}^{\#}(\underline{f}), j=2, \ldots, n$. De fato,

$$
\begin{aligned}
M_{j q_{0}}(\underline{\tilde{f}}) & =M_{j}(\tilde{\tilde{f}})\left(q_{0}\right)=\left.\left(L_{j U}^{\#}\right)\right|_{W}(\tilde{f})\left(q_{0}\right)= \\
& =L_{j}^{\#}(\hat{\tilde{f}} \circ \xi) \circ \xi^{-1}\left(0, q_{0}\right)=L_{j}^{\#}(\hat{\tilde{f}} \circ \xi)\left(p_{0}\right),
\end{aligned}
$$

mas

$$
\begin{aligned}
\frac{\partial(\hat{\tilde{f}} \circ \xi)}{\partial \xi_{k}}\left(p_{0}\right) & =\frac{\partial \hat{\tilde{f}}}{\partial e_{k}}\left(0, q_{0}\right)=\frac{\partial \tilde{f}}{\partial e_{k}}\left(q_{0}\right)=\frac{\partial\left(f \circ \xi^{-1}\right)}{\partial e_{k}}\left(\xi\left(p_{0}\right)\right), \\
L_{j}^{\#}(\hat{\tilde{f}} \circ \xi)\left(p_{0}\right) & =\sum_{k=2}^{N} a_{j k}\left(p_{0}\right) \frac{\partial(\hat{\tilde{f}} \circ \xi)}{\partial \xi_{k}}\left(p_{0}\right)= \\
& =\sum_{k=2}^{N} a_{j k}\left(p_{0}\right) \frac{\partial\left(f \circ \xi^{-1}\right)}{\partial e_{k}}\left(\xi\left(p_{0}\right)\right)=L_{j}^{\#}(f)\left(p_{0}\right),
\end{aligned}
$$

logo de 5.1 e 5.2 tem-se que

$$
M_{j q_{0}}(\underline{\tilde{f}})=L_{j}^{\#}(f)\left(p_{0}\right)=L_{j p_{0}}^{\#}(\underline{f})
$$

Por fim, se $\alpha_{2}, \ldots, \alpha_{n} \in \mathbb{C}$ e $q_{0} \in W$ forem tais que $\sum_{j=2}^{n} \alpha_{j} M_{j q_{0}}=0$ então $\sum_{j=2}^{n} \alpha_{j} L_{j p_{0}}^{\#}=0$, onde $p_{0}=\xi^{-1}\left(0, q_{0}\right)$, pois

$$
\sum_{j=2}^{n} \alpha_{j} L_{j p_{0}}^{\#}(\underline{f})=\sum_{j=2}^{n} \alpha_{j} M_{j q_{0}}(\underline{\tilde{f}})=0, \underline{f} \in C^{\infty}\left(p_{0}\right),
$$

então $\alpha_{j}=0, j=2, \ldots, n$, e segue que $\left\{M_{j q}\right\}_{j=2}^{n}$ é linearmente independente para cada $q \in W$. 
Defina $\mathcal{U}_{q} \doteq\left[M_{2 q}, \ldots, M_{n q}\right], q \in W$. Então

$$
\mathcal{U}=\cup_{p \in W} \mathcal{U}_{q} \subset \mathbb{C} T W
$$

é subfibrado vetorial complexo, iremos mostrar que $\mathcal{U}$ é uma estrutura involutiva.

Considere a aplicação

$$
\begin{aligned}
\psi: W & \rightarrow U \\
q & \mapsto \psi(q) \doteq(0, q) .
\end{aligned}
$$

Afirmamos que

$$
\left[M_{j}, M_{k}\right]=\sum_{\nu=2}^{n}\left(c_{j k}^{\nu} \circ \xi^{-1} \circ \psi\right) M_{\nu}, j, k=2, \ldots, n
$$

Primeiramente, note que

$$
\begin{aligned}
{\left.\left[L_{j}^{\#}, L_{k}^{\#}\right]_{U}\right|_{W} } & =\left.\left(\sum_{\nu=2}^{n} c_{j k}^{\nu} L_{\nu}^{\#}\right)_{U}\right|_{W}, \\
\left.\left(\sum_{\nu=2}^{n} c_{j k}^{\nu} L_{\nu}^{\#}\right)_{U}\right|_{W}(f)(q) & =\left(\sum_{\nu=2}^{n} c_{j k}^{\nu} L_{\nu}^{\#}\right)(\hat{f} \circ \xi)\left(\xi^{-1}(0, q)\right)= \\
& =\sum_{\nu=2}^{n} c_{j k}^{\nu}\left(\xi^{-1}(0, q)\right) L_{\nu}^{\#}(\hat{f} \circ \xi)\left(\xi^{-1}(0, q)\right)= \\
& =\sum_{\nu=2}^{n}\left(c_{j k}^{\nu} \circ \xi^{-1} \circ \psi\right)(q) M_{\nu}(f)(q),
\end{aligned}
$$

para $f \in C^{\infty}(W), q \in W, j, k=1, \ldots, n$, e então

$$
\left.\left(\sum_{\nu=2}^{n} c_{j k}^{\nu} L_{\nu}^{\#}\right)_{U}\right|_{W}=\sum_{\nu=2}^{n}\left(c_{j k}^{\nu} \circ \xi^{-1} \circ \psi\right) M_{\nu}
$$

Agora basta mostrar que

$$
\left.\left[L_{j}^{\#}, L_{k}^{\#}\right]_{U}\right|_{W}=\left[M_{j}, M_{k}\right], j, k=2, \ldots, n
$$

Observe que

$$
\begin{aligned}
\left(L_{j}^{\#}\left(L_{k}^{\#} f\right)\right)(p) & =L_{j}^{\#}\left(\sum_{\nu=2}^{N} a_{k \nu} \frac{\partial f}{\partial \xi_{\nu}}\right)(p)= \\
& =\sum_{l, \nu=2}^{N} a_{j l}\left(\frac{\partial a_{k \nu}}{\partial \xi_{l}} \frac{\partial f}{\partial \xi_{\nu}}+a_{k \nu} \frac{\partial^{2} f}{\partial \xi_{l} \xi_{\nu}}\right),
\end{aligned}
$$

e por 2.4 tem-se

$$
\left[L_{j}^{\#}, L_{k}^{\#}\right](f)=L_{j}^{\#}\left(L_{k}^{\#} f\right)-L_{k}^{\#}\left(L_{j}^{\#} f\right)=\sum_{l, \nu=2}^{N}\left(a_{j l} \frac{\partial a_{k \nu}}{\partial \xi_{l}} \frac{\partial f}{\partial \xi_{\nu}}-a_{k \nu} \frac{\partial a_{j l}}{\partial \xi_{\nu}} \frac{\partial f}{\partial \xi_{l}}\right)
$$


para $f \in C^{\infty}(V)$ e $j, k=2, \ldots, n$. Assim

$$
\begin{aligned}
{\left.\left[L_{j}^{\#}, L_{k}^{\#}\right]_{U}\right|_{W}(f)(q) } & =\left[L_{j}^{\#}, L_{k}^{\#}\right](\hat{f} \circ \xi)\left(\xi^{-1}(0, q)\right) \\
& =\sum_{l, \nu=2}^{N}\left(a_{j l} \circ \xi^{-1} \circ \psi\right)(q)\left(\frac{\partial a_{k \nu}}{\partial \xi_{l}} \circ \xi^{-1} \circ \psi\right)(q){\frac{\partial}{\partial \xi_{\nu}}}_{{ }_{W}}(f)(q)- \\
& -\left.\left(a_{k \nu} \circ \xi^{-1} \circ \psi\right)(q)\left(\frac{\partial a_{j l}}{\partial \xi_{\nu}} \circ \xi^{-1} \circ \psi\right)(q) \frac{\partial}{\partial \xi_{l}}\right|_{W}(f)(q),
\end{aligned}
$$

$q \in W, j, k=2, \ldots, n$.

Por outro lado,

$$
\begin{aligned}
M_{j}\left(M_{k} f\right)(q) & =\left(\left.\sum_{l=2}^{N} a_{j l} \circ \xi^{-1} \circ \psi \frac{\partial}{\partial \xi_{l}}\right|_{W}\right)\left(\left.\sum_{\nu=2}^{N} a_{k \nu} \circ \xi^{-1} \circ \psi{\frac{\partial}{\partial \xi_{\nu}}}_{W}\right|_{W}(f)\right)(q) \\
& =\sum_{l, \nu=2}^{N}\left(a_{j l} \circ \xi^{-1} \circ \psi\right)(q)\left(\left.\left.\frac{\partial}{\partial \xi_{l}}\right|_{W}\left(a_{k \nu} \circ \xi^{-1} \circ \psi\right)(q) \frac{\partial}{\partial \xi_{\nu}}\right|_{W}(f)(q)\right)+ \\
& +\left.\left(a_{k \nu} \circ \xi^{-1} \circ \psi\right)(q) \frac{\partial}{\partial \xi_{l}}\right|_{W}\left(\left.\frac{\partial}{\partial \xi_{\nu}}\right|_{W}(f)\right)(q),
\end{aligned}
$$

para $q \in W, j, k=2, \ldots, n$.

Observação 5.2.5. Afirmamos que

$$
\begin{aligned}
\left.\frac{\partial}{\partial \xi_{l}}\right|_{W}\left(\left.\frac{\partial}{\partial \xi_{\nu}}\right|_{W}(f)\right) & =\left.\frac{\partial}{\partial \xi_{\nu}}\right|_{W}\left(\left.\frac{\partial}{\partial \xi_{l}}\right|_{W}(f)\right), f \in C^{\infty}(W) \\
\left.\frac{\partial}{\partial \xi_{l}}\right|_{W}\left(a_{k \nu} \circ \xi^{-1} \circ \psi\right) & =\frac{\partial a_{k \nu}}{\partial \xi_{l}} \circ \xi^{-1} \circ, \psi
\end{aligned}
$$

$k, \nu, l=2, \ldots, n$.

Com efeito, observe que

$$
\left.\frac{\partial}{\partial \xi_{l}}\right|_{W}\left(\left.{\frac{\partial}{\partial \xi_{\nu}}}_{U}\right|_{W}(f)\right)=\left.{\frac{\partial}{\partial \xi_{l}}}_{U}\right|_{W}\left(\frac{\partial(\hat{f} \circ \xi)}{\partial \xi_{\nu}} \circ \xi^{-1} \circ \psi\right),
$$

denote

$$
g(q)=\left(\frac{\partial(\hat{f} \circ \xi)}{\partial \xi_{\nu}} \circ \xi^{-1} \circ \psi\right)(q), q \in W
$$

Então

$$
\left.\frac{\partial}{\partial \xi_{l}}\right|_{W}\left(\left.\frac{\partial}{\partial \xi_{\nu}}\right|_{W}(f)\right)=\frac{\partial(\hat{g} \circ \xi)}{\partial \xi_{l}} \circ \xi^{-1} \circ \psi,
$$

mas

$$
\begin{aligned}
\frac{\partial(\hat{g} \circ \xi)}{\partial \xi_{l}} \circ \xi^{-1} \circ \psi(q) & =\frac{\partial \hat{g}}{\partial e_{l}}(0, q)=\frac{\partial g}{\partial e_{l}}(q)=\frac{\partial}{\partial e_{l}}\left(\frac{\partial(\hat{f} \circ \xi)}{\partial \xi_{\nu}} \circ \xi^{-1} \circ \psi\right)(q) \\
& =\frac{\partial}{\partial e_{l}}\left(\frac{\partial \hat{f}}{\partial e_{\nu}} \circ \xi^{-1}\right)(0, q)=\frac{\partial}{\partial e_{l}}\left(\frac{\partial f}{\partial e_{\nu}}\right)(q)
\end{aligned}
$$


Assim

$$
\begin{aligned}
& \left.\frac{\partial}{\partial \xi_{l}}\right|_{W}\left(\left.\frac{\partial}{\partial \xi_{\nu}}\right|_{W}(f)\right)=\frac{\partial}{\partial e_{l}}\left(\frac{\partial f}{\partial e_{\nu}}\right) \\
& \left.\frac{\partial}{\partial \xi_{\nu}}\right|_{W}\left(\left.\frac{\partial}{\partial \xi_{l}}\right|_{W}(f)\right)=\frac{\partial}{\partial e_{\nu}}\left(\frac{\partial f}{\partial e_{l}}\right)
\end{aligned}
$$

e como

$$
\frac{\partial}{\partial e_{l}}\left(\frac{\partial f}{\partial e_{\nu}}\right)=\frac{\partial}{\partial e_{\nu}}\left(\frac{\partial f}{\partial e_{l}}\right)
$$

concluímos 5.3.

Demonstremos 5.4. Note que

$$
\frac{\partial a_{k \nu}}{\partial \xi_{l}} \circ \xi^{-1} \circ \psi(q)=\frac{\partial\left(a_{k \nu} \circ \xi^{-1}\right)}{\partial e_{l}}(0, q),
$$

denote

$$
g(q) \doteq\left(a_{k \nu} \circ \xi^{-1} \circ \psi\right)(q), q \in W .
$$

Assim tem-se que

$$
\begin{aligned}
\left.\frac{\partial}{\partial \xi_{l U}}\right|_{W}\left(a_{k \nu} \circ \xi^{-1} \circ \psi\right)(q) & =\frac{\partial(\hat{g} \circ \xi)}{\partial \xi_{l}}\left(\xi^{-1}(0, q)\right)=\frac{\partial \hat{g}}{\partial e_{l}}(0, q)= \\
& =\frac{\partial g}{\partial e_{l}}(q)=\frac{\partial\left(a_{k \nu} \circ \xi^{-1} \circ \psi\right)}{\partial e_{l}}(q),
\end{aligned}
$$

por outro lado,

$$
\begin{aligned}
\frac{\partial\left(a_{k \nu} \circ \xi^{-1} \circ \psi\right)}{\partial e_{l}}(q) & =\lim _{h \rightarrow 0} \frac{\left(a_{k \nu} \circ \xi^{-1} \circ \psi\right)\left(q+h e_{l}\right)-a_{k \nu} \circ \xi^{-1} \circ \psi(q)}{h}= \\
& =\lim _{h \rightarrow 0} \frac{\left(a_{k \nu} \circ \xi^{-1}\right)\left((0, q)+h e_{l}\right)-a_{k \nu} \circ \xi^{-1}(0, q)}{h}= \\
& =\frac{\partial\left(a_{k \nu} \circ \xi^{-1}\right)}{\partial e_{l}}(0, q),
\end{aligned}
$$

de 5.5 e 5.6 concluímos 5.4 .

Agora de 5.3 e 5.4 obtemos

$$
\begin{aligned}
{\left[M_{j}, M_{k}\right](f) } & =\left.\left.\sum_{l, \nu=2}^{N} a_{j l} \circ \xi^{-1} \circ \psi \frac{\partial}{\partial \xi_{l}}\right|_{W}\left(a_{k \nu} \circ \xi^{-1} \circ \psi\right) \frac{\partial}{\partial \xi_{\nu}}\right|_{W}(f)- \\
& -\left.\left.a_{k \nu} \circ \xi^{-1} \circ \psi \frac{\partial}{\partial \xi_{\nu}}\right|_{W}\left(a_{j l} \circ \xi^{-1} \circ \psi\right) \frac{\partial}{\partial \xi_{l}}\right|_{W}(f)= \\
& =\left.\sum_{l, \nu=2}^{N} a_{j l} \circ \xi^{-1} \circ \psi \frac{\partial a_{k \nu}}{\partial \xi_{l}} \circ \xi^{-1} \circ \psi \frac{\partial}{\partial \xi_{\nu}}\right|_{W}(f)- \\
& -\left.a_{k \nu} \circ \xi^{-1} \circ \psi \frac{\partial a_{j l}}{\partial \xi_{\nu}} \circ \xi^{-1} \circ \psi \frac{\partial}{\partial \xi_{l}}\right|_{W}(f) \\
& =\left.\left[L_{j}^{\#}, L_{k}^{\#}\right]_{U}\right|_{W}(f)= \\
& =\sum_{\nu=2}^{n} c_{j k}^{\nu} \circ \xi^{-1} \circ \psi M_{\nu},
\end{aligned}
$$


para $j, k=2, \ldots, n$ e portanto $\mathcal{U}$ é uma estrutura involutiva.

Aplicando a hipótese de indução, obtemos um sistema de coordenadas locais $\phi \doteq$ $\left(\phi_{2}, \ldots, \phi_{N}\right)$ em torno da origem tal que

$$
\mathcal{U}_{q}=\left[\left(\partial / \partial \phi_{2}\right)_{q}, \ldots,\left(\partial / \partial \phi_{N}\right)_{q}\right], q \in W
$$

Em particular,

$$
\phi: W \rightarrow \phi(W) \subset \mathbb{R}^{N-1}
$$

é difeomorfismo e denote $Z \doteq \phi(W)$.

Defina

$$
\begin{aligned}
\eta: U & \rightarrow \eta(U) \\
q & \mapsto \eta(q) \doteq\left(q_{1}, \phi_{2}\left(q_{2}, \ldots, q_{N}\right), \ldots, \phi_{N}\left(q_{2}, \ldots, q_{N}\right)\right)
\end{aligned}
$$

$\operatorname{assim} D \eta(q)=$

$$
\left(\begin{array}{cccc}
1 & 0 & \cdots & 0 \\
0 & \frac{\partial \phi_{2}}{\partial e_{2}}(q) & \cdots & \frac{\partial \phi_{2}}{\partial e_{N}}(q) \\
\vdots & \vdots & \ddots & \vdots \\
0 & \frac{\partial \phi_{N}}{\partial e_{2}}(q) & \cdots & \frac{\partial \phi_{N}}{\partial e_{N}}(q)
\end{array}\right)
$$

e então det $D \eta(q)=\operatorname{det} D \phi(q) \neq 0$, por fim, pelo Teorema da Função Inversa, concluímos que $\eta$ é difeomorfismo. Observe também que $\eta(U)=\eta(\xi(V))=(-\delta, \delta) \times \phi(W)=(-\delta, \delta) \times$ $Z$. Defina

$$
\begin{aligned}
h: V & \rightarrow h(V) \\
q & \mapsto h(q) \doteq \eta(\xi(q)) .
\end{aligned}
$$

Em particular, tem-se que $h$ é difeomorfismo e $h_{1}=\xi_{1}$.

Observação 5.2.6. Afirmamos que

$$
\begin{aligned}
\frac{\partial}{\partial h_{1}} & =\frac{\partial}{\partial \xi_{1}} \\
\left.\frac{\partial}{\partial h_{l}}\right|_{W} & =\frac{\partial}{\partial \phi_{l}}, l=2, \ldots, N .
\end{aligned}
$$

De fato,

$$
\begin{aligned}
\frac{\partial f}{\partial \xi_{1}}(q) & =\frac{\partial\left(f \circ \xi^{-1}\right)}{\partial e_{1}}(\xi(q)) \\
\frac{\partial f}{\partial h_{1}}(q) & =\frac{\partial\left(f \circ h^{-1}\right)}{\partial e_{1}}(h(q))
\end{aligned}
$$

Seja $\tilde{q} \doteq \xi^{-1}\left(\xi(q)+k e_{1}\right) \in V$. Assim

$$
\begin{aligned}
h(\tilde{q}) & =\eta(\xi(\tilde{q}))=\eta\left(\xi(q)+k e_{1}\right)=\eta\left(\xi_{1}(q)+k, \xi_{2}(q), \ldots, \xi_{N}(q)\right)= \\
& =\left(\xi_{1}(q)+k, \phi\left(\xi_{2}(q), \ldots, \xi_{N}(q)\right)\right)=\eta(\xi(q))+k e_{1}=h(q)+k e_{1}
\end{aligned}
$$


então

$$
\begin{aligned}
\frac{\partial\left(f \circ \xi^{-1}\right)}{\partial e_{1}}(\xi(q)) & =\lim _{k \rightarrow 0} \frac{f \circ \xi^{-1}\left(\xi(q)+k e_{1}\right)-f \circ \xi^{-1}(\xi(q))}{k} \\
& =\lim _{k \rightarrow 0} \frac{f(\tilde{q})-f(q)}{k}=\lim _{k \rightarrow 0} \frac{f \circ h^{-1}(h(\tilde{q}))-f \circ h^{-1}(h(q))}{k}= \\
& =\lim _{k \rightarrow 0} \frac{f \circ h^{-1}\left(h(q)+k e_{1}\right)-f \circ h^{-1}(h(q))}{k}= \\
& =\frac{\partial\left(f \circ h^{-1}\right)}{\partial e_{1}}(h(q)) .
\end{aligned}
$$

Para provarmos 5.7, note que

$$
h\left(\xi^{-1}(0, q)\right)=\eta\left(\xi\left(\xi^{-1}(0, q)\right)\right)=\eta(0, q)=(0, \phi(q))
$$

$e$

$$
\begin{aligned}
\left.\frac{\partial}{\partial h_{l U}}\right|_{W}(f)(q) & =\frac{\partial(\hat{f} \circ \xi)}{\partial h_{l}}\left(\xi^{-1}(0, q)\right)= \\
& =\frac{\partial\left(\hat{f} \circ \xi \circ h^{-1}\right)}{\partial e_{l}}\left(h \circ \xi^{-1}(0, q)\right) \\
& =\frac{\partial\left(\hat{f} \circ \xi \circ h^{-1}\right)}{\partial e_{l}}(0, \phi(q)), \\
\frac{\partial f}{\partial \phi_{l}}(q) & =\frac{\partial\left(f \circ \phi^{-1}\right)}{\partial e_{l}}(\phi(q)) .
\end{aligned}
$$

Considere a aplicação

$$
\begin{aligned}
\pi_{1} \times \phi^{-1}: U & \rightarrow \mathbb{R}^{N} \\
q & \mapsto\left(\pi_{1} \times \phi^{-1}\right)(q) \doteq\left(q_{1}, \phi^{-1}\left(q_{2}, \ldots, q_{N}\right)\right)
\end{aligned}
$$

e observe que

$$
\begin{aligned}
\left(\pi_{1} \times \phi^{-1}\right)(\eta(q)) & =\left(q_{1}, \phi^{-1}\left(\phi\left(q_{2}, \ldots, q_{N}\right)\right)\right)=q, \\
\eta\left(\pi_{1} \times \phi^{-1}(q)\right) & =\left(q_{1}, \phi\left(\phi^{-1}\left(q_{2}, \ldots, q_{N}\right)\right)\right)=q,
\end{aligned}
$$

ou seja, $\eta^{-1}=\pi_{1} \times \phi^{-1}$.

Logo

$$
\hat{f} \circ \eta^{-1}(q)=\hat{f}\left(q_{1}, \ldots, \phi^{-1}\left(q_{2}, \ldots, q_{N}\right)\right)=f\left(\phi^{-1}\left(q_{2}, \ldots, q_{N}\right)\right)
$$

e

$$
\begin{aligned}
\frac{\partial\left(\hat{f} \circ \xi \circ h^{-1}\right)}{\partial e_{l}}\left(h \circ \xi^{-1}(0, q)\right) & =\frac{\partial\left(\hat{f} \circ \xi \circ h^{-1}\right)}{\partial e_{l}}((0, \phi(q)))= \\
& =\frac{\partial\left(\hat{f} \circ \eta^{-1}\right)}{\partial e_{l}}(0, \phi(q))= \\
& =\lim _{k \rightarrow 0} \frac{\hat{f} \circ \eta^{-1}\left((0, \phi(q))+k e_{l}\right)-\hat{f} \circ \eta^{-1}(0, \phi(q))}{k}= \\
& =\lim _{k \rightarrow 0} \frac{f \circ \phi^{-1}\left(\phi(q)+k e_{l}\right)-f \circ \phi^{-1}(\phi(q))}{k}
\end{aligned}
$$


completando a demonstração.

Assim

$$
L_{1}^{\#}=\frac{\partial}{\partial \xi_{1}}=\frac{\partial}{\partial h_{1}}
$$

e existem funções $b_{j k} \in C^{\infty}(V), j=2, \ldots, n, k=1, \ldots, N$, tais que

$$
L_{j}^{\#}=\sum_{k=1}^{N} b_{j k} \frac{\partial}{\partial h_{k}} \text {. }
$$

Por outro lado,

$$
b_{j 1}=L_{j}^{\#}\left(h_{1}\right)=L_{j}^{\#}\left(\xi_{1}\right)=0, j=2, \ldots, n,
$$

então

$$
L_{j}^{\#}=\sum_{k=2}^{N} b_{j k} \frac{\partial}{\partial h_{k}}
$$

Além disso,

$$
M_{j}=\left.L_{j U}^{\#}\right|_{W}=\left.\sum_{k=2}^{N}\left(b_{j k} \circ \xi^{-1} \circ \psi\right) \frac{\partial}{\partial h_{k} U}\right|_{W}=\sum_{k=2}^{N}\left(b_{j k} \circ \xi^{-1} \circ \psi\right) \frac{\partial}{\partial \phi_{k}},
$$

$j=2, \ldots, n$. Como, para cada $q \in W$, tem-se que $M_{j q} \in \mathcal{U}_{q}, j=2, \ldots, n$, então existem $\lambda_{2}, \ldots, \lambda_{n} \in \mathbb{C}$ tais que

mas

$$
M_{j q}=\sum_{k=2}^{n} \lambda_{k} \frac{\partial}{\partial \phi_{k q}}
$$

$$
M_{j q}=\sum_{k=2}^{N}\left(b_{j k} \circ \xi^{-1} \circ \psi\right)(q) \frac{\partial}{\partial \phi_{k}}
$$

em outras palavras,

$$
b_{j k} \circ \xi^{-1}(0, q)=0,(0, q) \in U, j=2, \ldots, n, k=n+1, \ldots, N .
$$

Vamos mostrar que $b_{j k}=0, j=2, \ldots, n, k=n+1, \ldots, N$. Inicialmente, temos que

mas

$$
\left[L_{1}^{\#}, L_{j}^{\#}\right]=\sum_{\nu=1}^{n} c_{1 j}^{\nu} L_{\nu}^{\#}
$$

$$
\begin{aligned}
{\left[L_{1}^{\#}, L_{j}^{\#}\right](g) } & =\frac{\partial}{\partial h_{1}}\left(\sum_{k=2}^{N} b_{j k} \frac{\partial g}{\partial h_{k}}\right)-\sum_{k=2}^{N} b_{j k} \frac{\partial}{\partial h_{k}}\left(\frac{\partial g}{\partial h_{1}}\right)= \\
& =\sum_{k=2}^{N}\left(\frac{\partial b_{j k}}{\partial h_{1}} \frac{\partial g}{\partial h_{k}}+b_{j k} \frac{\partial}{\partial h_{1}} \frac{\partial g}{\partial h_{k}}-b_{j k} \frac{\partial}{\partial h_{k}} \frac{\partial g}{\partial h_{1}}\right)= \\
& =\sum_{k=2}^{N} \frac{\partial b_{j k}}{\partial h_{1}} \frac{\partial g}{\partial h_{k}}, g \in C^{\infty}(V),
\end{aligned}
$$

ou seja,

$$
\left[L_{1}^{\#}, L_{j}^{\#}\right]=\sum_{k=2}^{N} \frac{\partial b_{j k}}{\partial h_{1}} \frac{\partial}{\partial h_{k}} .
$$


Como

$$
\begin{aligned}
& L_{\nu}^{\#}\left(h_{l}\right)=\sum_{k=2}^{N} b_{\nu k} \frac{\partial h_{l}}{\partial h_{k}}=b_{\nu l}, \nu=2, \ldots, n, l=2, \ldots, N, \\
& \sum_{\nu=1}^{n} c_{1 j}^{\nu} L_{\nu}^{\#}\left(h_{l}\right)= c_{1 j}^{1} L_{1}^{\#}\left(h_{l}\right)+\sum_{\nu=2}^{n} c_{1 j}^{\nu} L_{\nu}^{\#}\left(h_{l}\right)= \\
&= \sum_{\nu=2}^{n} c_{1 j}^{\nu} b_{\nu l}, \\
& {\left[L_{1}^{\#}, L_{j}^{\#}\right]\left(h_{l}\right)=\frac{\partial b_{j l}}{\partial h_{1}} }
\end{aligned}
$$

obtemos

$$
\frac{\partial b_{j l}}{\partial h_{1}}=\sum_{\nu=2}^{n} c_{1 j}^{\nu} b_{\nu l}
$$

Além disso,

$$
\begin{aligned}
\frac{\partial b_{j k}}{\partial h_{1}}\left(\xi^{-1}(q)\right) & =\frac{\partial\left(b_{j k} \circ h^{-1}\right)}{\partial e_{1}}\left(h \circ \xi^{-1}(q)\right)=\frac{\partial\left(b_{j k} \circ h^{-1}\right)}{\partial e_{1}}\left(\left(q_{1}, \phi\left(q_{2}, \ldots, q_{N}\right)\right)\right. \\
& =\lim _{l \rightarrow 0} \frac{b_{j k} \circ h^{-1}\left(\left(q_{1}, \phi\left(q_{2}, \ldots, \phi\left(q_{n}\right)\right)\right)+l e_{1}\right)-b_{j k} \circ h^{-1}\left(q_{1}, \phi\left(q_{2}, \ldots, q_{N}\right)\right)}{l} \\
& =\lim _{l \rightarrow 0} \frac{b_{j k} \circ \xi^{-1}\left(q+l e_{1}\right)-b_{j k} \circ \xi^{-1}(q)}{l} \\
& =\frac{\partial\left(b_{j k} \circ \xi^{-1}\right)}{\partial e_{1}}(q), q \in U,
\end{aligned}
$$

pois $h^{-1}=\xi^{-1} \circ\left(\pi \times \phi^{-1}\right)$. Assim

$$
\frac{\partial\left(b_{j l} \circ \xi^{-1}\right)}{\partial e_{1}}=\frac{\partial b_{k l}}{\partial h_{1}} \circ \xi^{-1}=\sum_{\nu=2}^{N}\left(c_{1 j}^{\nu} \circ \xi^{-1}\right)\left(b_{\nu l} \circ \xi^{-1}\right) .
$$

Denote

$$
\begin{gathered}
B_{j l} \doteq b_{j l} \circ \xi^{-1} \\
C_{1 j}^{\nu} \doteq c_{1 j}^{\nu} \circ \xi^{-1}
\end{gathered}
$$

e concluímos que

$$
\begin{aligned}
\frac{\partial B_{j l}}{\partial e_{1}} & =\sum_{\nu=2}^{N} C_{1 j}^{\nu} B_{\nu l} \\
B_{j l}(0, q) & =0, q \in W, j=2, \ldots, n, l=n+1, \ldots, N .
\end{aligned}
$$

Defina, para cada $q \in W, B_{j l q}(t) \doteq B_{j l}(t, q), C_{1 j q}^{\nu}(t) \doteq C_{1 j}^{\nu}(t, q), t \in(-\delta, \delta)$. Reescrevendo o sistema acima, tem-se que

$$
\begin{aligned}
\frac{d B_{j l q}}{d t}(t) & =\sum_{\nu=2}^{N} C_{1 j q}^{\nu}(t) B_{\nu l q}(t), t \in(-\delta, \delta) \\
B_{j l q}(0) & =0, j=2, \ldots, n, l=n+1, \ldots, N,
\end{aligned}
$$


que é uma equação diferencial ordinária que possui solução única, visto que $C_{1 j q}^{\nu} \in$ $C^{\infty}(-\delta, \delta)$. Como $B_{j l q}(t)=0$ é solução, concluímos que

$$
B_{j l}(t, q)=B_{j l q}(t)=0, t \in(-\delta, \delta), q \in W, j=2, \ldots, n, l=n+1, \ldots, N,
$$

ou seja,

$$
\begin{aligned}
b_{j l} \circ \xi^{-1} & =0, j=2, \ldots, n, l=n+1, \ldots, N, \\
b_{j l} & =0, j=2, \ldots, n, l=n+1, \ldots, N .
\end{aligned}
$$

Dessa forma,

$$
\begin{aligned}
L_{1}^{\#} & =\frac{\partial}{\partial h_{1}}, \\
L_{j}^{\#} & =\sum_{k=2}^{N} b_{j k} \frac{\partial}{\partial h_{k}}=\sum_{k=2}^{n} b_{j k} \frac{\partial}{\partial h_{k}}, j=2, \ldots, n,
\end{aligned}
$$

e assim

$$
\mathcal{V}_{p}=\left[\frac{\partial}{\partial h_{1}}, \ldots, \frac{\partial}{\partial h_{n p}}\right], p \in V
$$

Teorema 5.2.7 (Versão para variedades suaves). Sejam $\Omega$ variedade suave de dimensão $N$ e $U \subset \Omega$ um aberto não vazio. Se $L_{1}, \ldots, L_{n} \in \mathfrak{X}(U)$ são campos reais tais que $\left\{L_{j p}\right\}_{j=1}^{n}$ é linearmente independente, para cada $p \in U$. Se $\mathcal{V} \doteq \cup_{p \in U} \mathcal{V}_{p}$, em que

$$
\mathcal{V}_{p}=\left[L_{1 p}, \ldots, L_{n p}\right]
$$

for involutiva, então para cada $p_{0} \in U$ existe sistema de coordenadas locais $(W, y)$, com $p_{0} \in W$, tal que

$$
\mathcal{V}_{p}=\left[\frac{\partial}{\partial y_{1}}, \ldots, \frac{\partial}{\partial y_{n}}\right], p \in W
$$

Demonstração. Seja $p_{0} \in U$ e considere $(V, x)$ um sistema de coordenadas locais tal que $p_{0} \in V$ e $x\left(p_{0}\right)=0$. Defina a aplicação

$$
\begin{aligned}
\psi: \mathfrak{X}(V) & \rightarrow \mathfrak{X}(x(V)) \\
L & \mapsto \psi(L),
\end{aligned}
$$

onde $\psi(L) f \doteq L(f \circ x) \circ x^{-1}, f \in C^{\infty}(x(V))$.

Note que $\psi$ é $\mathbb{C}$-linear e é bijeção. De fato, se $\psi(L)=\psi(M), L, M \in \mathfrak{X}(V)$, então

$$
\begin{aligned}
L(f \circ x) \circ x^{-1} & =M(f \circ x) \circ x^{-1} \\
\Rightarrow L(f \circ x) & =M(f \circ x) \\
\Rightarrow L(f)=L\left(f \circ x^{-1} \circ x\right) & =M\left(f \circ x^{-1} \circ x\right)=M(f),
\end{aligned}
$$


$f \in C^{\infty}(V)$, então $L=M$.

Se $\tilde{L} \in \mathfrak{X}(x(V))$, defina $L f \doteq \tilde{L}\left(f \circ x^{-1}\right) \circ x, f \in C^{\infty}(V)$. Assim

$$
\psi(L) f=L(f \circ x) \circ x^{-1}=\tilde{L}\left(f \circ x \circ x^{-1}\right) \circ x \circ x^{-1}=\tilde{L} f, f \in C^{\infty}(x(V)),
$$

ou seja, $\psi(L)=\tilde{L}$ e portanto $\psi$ é bijeção.

Se $L_{1}, L_{2} \in \mathfrak{X}(V)$ e $\lambda \in \mathbb{C}$, então

$$
\begin{aligned}
\psi\left(L_{1}+\lambda L_{2}\right) f & =\left[\left(L_{1}+\lambda L_{2}\right)(f \circ x)\right] \circ x^{-1}= \\
& =\left[L_{1}(f \circ x)+L_{2}(f \circ x)\right] \circ x^{-1}= \\
& =L_{1}(f \circ x) \circ x^{-1}+\lambda L_{2}(f \circ x) \circ x^{-1}= \\
& =\left(\psi\left(L_{1}\right)+\lambda \psi\left(L_{2}\right)\right) f, f \in C^{\infty}(x(V)),
\end{aligned}
$$

ou seja, $\psi\left(L_{1}+\lambda L_{2}\right)=\psi\left(L_{1}\right)+\lambda \psi\left(L_{2}\right)$. Portanto $\psi$ é um isomorfismo entre os $\mathbb{C}$-espaços vetoriais $\mathfrak{X}(V)$ e $\mathfrak{X}(x(V))$.

Seja $\phi \doteq \psi^{-1}$. Assim

$$
\phi: \mathfrak{X}(x(V)) \rightarrow \mathfrak{X}(V)
$$

é tal que

$$
\psi(\phi(\tilde{L}))=\tilde{L} \Leftrightarrow \tilde{L} f=\psi(\phi(\tilde{L})) f=\phi(\tilde{L})(f \circ x) \circ x^{-1}, f \in C^{\infty}(x(V)),
$$

então

$$
\phi(\tilde{L}) f=\phi(\tilde{L})\left(f \circ x^{-1} \circ x\right)=\tilde{L}\left(f \circ x^{-1}\right) \circ x, f \in C^{\infty}(V) .
$$

Observe que o isomorfismo $\psi$ induz, para cada $p \in V$, a aplicação

$$
\begin{aligned}
\psi_{p}: \mathbb{C} T_{p} V & \rightarrow \mathbb{C} T_{q} x(V) \\
v & \mapsto \psi_{p}(v) \doteq \psi(L)_{q}
\end{aligned}
$$

onde $q=x(p), L \in \mathfrak{X}(V)$ é tal que $v=L_{p}$.

Analogamente tem-se que, para cada $p \in V, \psi_{p}$ é isomorfismo com $\left(\psi_{p}\right)^{-1}=\phi_{q}$ onde $q=x(p)$ e $\phi_{q}(v)=\phi(L)_{p}$, com $L \in \mathfrak{X}(x(V))$ tal que $v=L_{q}$.

Defina, para cada $j \in\{1, \ldots, n\}$, os campos $\tilde{L}_{j} \doteq \psi\left(L_{j}\right)$. Assim $\tilde{L}_{j q}=\psi\left(L_{j}\right)_{q}=$ $\psi_{p}\left(L_{j p}\right), j=1, \ldots, n, q \in x(V), p=x^{-1}(q)$.

Afirmamos que $\left\{\tilde{L}_{j 1}\right\}_{j=1}^{n}$ é linearmente independente, $\forall q \in x(V)$. Com efeito, se $\left\{\lambda_{j}\right\}_{j=1}^{n} \subset \mathbb{C}$ e $q \in x(V)$ forem tais que $\sum_{j=1}^{n} \lambda_{j} \tilde{L}_{j q}=0$ e assim

$$
\sum_{j=1}^{n} \lambda_{j} \psi_{p}\left(L_{j p}\right)=0 \Rightarrow \psi_{p}\left(\sum_{j=1}^{n} \lambda_{j} L_{j p}\right)=0 \Rightarrow \sum_{j=1}^{n} \lambda_{j} L_{j p}=0,
$$

então $\lambda_{j}=0, j=1, \ldots, n$. 
Considere $\mathcal{U}=\cup_{q \in x(V)} \mathcal{U}_{q}$, onde $\mathcal{U}_{q}=\left[\tilde{L}_{1 q}, \ldots, \tilde{L}_{n q}\right], q \in x(V)$. Assim $\mathcal{U} \subset \mathbb{C} T x(V)$ é subfibrado vetorial complexo $n$-dimensional. Observe que

$$
\begin{aligned}
& \overline{\tilde{L}}_{j} f=\overline{\tilde{L}_{j}(\bar{f})}=\overline{L_{j}(\bar{f} \circ x) \circ x^{-1}}=\overline{L_{j}(\overline{f \circ x})} \circ x^{-1}= \\
& =\bar{L}_{j}(f \circ x) \circ x^{-1}=L_{j}(f \circ x) \circ x^{-1}= \\
& =\tilde{L}_{j} f, f \in C^{\infty}(x(V)) \text {, }
\end{aligned}
$$

$\operatorname{assim} \tilde{L}_{j}, j=1, \ldots, n$, são campos reais.

Vamos mostrar que $\mathcal{U}$ é uma estrutura involutiva. Sejam $\tilde{L}, \tilde{M} \in \mathfrak{X}(x(V))$ seções de $\mathcal{U}$ sobre $x(V)$. Tome $L \doteq \phi(\tilde{L}), M \doteq \phi(\tilde{M}) \in \mathfrak{X}(V)$. Observe que, se $p \in V$ e $q=$ $x(p) \in x(V)$, então existem $a_{1}, \ldots, a_{n} \in \mathbb{C}$ tais que

$$
\begin{aligned}
\tilde{L}_{q} & =\sum_{j=1}^{n} a_{j} \tilde{L}_{j q}=\sum_{j=1}^{n} a_{j} \psi_{p}\left(L_{j p}\right)= \\
& =\psi_{p}\left(\sum_{j=1}^{n} a_{j} L_{j p}\right)
\end{aligned}
$$

ou seja,

$$
L_{p}=\sum_{j=1}^{n} a_{j} L_{j p} .
$$

Analogamente, existem $b_{1}, \ldots, b_{n} \in \mathbb{C}$ tais que

$$
\tilde{M}_{q}=\sum_{j=1}^{n} b_{j} \tilde{L}_{j q}
$$

e obtemos que

$$
M_{p}=\sum_{j=1}^{n} b_{j} L_{j p},
$$

ou seja, $L$ e $M$ são seções de $\mathcal{V}$ sobre $V$.

Assim, para cada $q \in x(V)$, existem $c_{1}, \ldots, c_{n} \in \mathbb{C}$ tais que

$$
[L, M]_{p}=\sum_{j=1}^{n} c_{j} L_{j p}, p=x^{-1}(q) .
$$

Observe que

$$
\psi_{p}\left([L, M]_{p}\right)=\sum_{j=1}^{n} c_{j} \tilde{L}_{j q},
$$

vamos mostrar que $[\tilde{L}, \tilde{M}]_{q}=\psi_{p}\left([L, M]_{p}\right)$ e assim teremos provado que $\mathcal{U}$ é de fato involutiva.

Note que

$$
\begin{aligned}
\psi([L, M]) f & =[L, M](f \circ x) \circ x^{-1}=(L(M(f \circ x))-M(L(f \circ x))) \circ x^{-1}= \\
& =L\left(M(f \circ x) \circ x^{-1} \circ x\right) \circ x^{-1}-M\left(L(f \circ x) \circ x^{-1} \circ x\right) \circ x^{-1}= \\
& =\tilde{L}\left(M(f \circ x) \circ x^{-1}\right)-\tilde{M}\left(L(f \circ x) \circ x^{-1}\right)= \\
& =\tilde{L}(\tilde{M} f)-\tilde{M}(\tilde{L} f)=[\tilde{L}, \tilde{M}] f, f \in C^{\infty}(x(V)),
\end{aligned}
$$


ou seja, $\psi([L, M])=[\tilde{L}, \tilde{M}]$.

Portanto

$$
\psi_{p}\left([L, M]_{p}\right)=\psi([L, M])_{q}=[\tilde{L}, \tilde{M}]_{q}
$$

e concluímos que $\mathcal{U}$ é uma estrutura involutiva.

Como $\mathcal{U} \subset \mathbb{C} T x(V)$ é uma estrutura involutiva gerada por campos reais e $x(V) \subset$ $\mathbb{R}^{N}$ é aberto não vazio, pelo Teorema 5.2 .1 , existe $(\tilde{W}, \tilde{y})$ sistema de coordenadas locais com $0 \in \tilde{W} \subset x(V)$ tal que

$$
\mathcal{U}_{q}=\left[\frac{\partial}{\partial \tilde{y}_{1}}, \ldots, \frac{\partial}{\partial \tilde{y}_{n_{q}}}\right], \forall q \in \tilde{W} .
$$

Defina $W \doteq x^{-1}(\tilde{W}) \subset V$ e $y \doteq \tilde{y} \circ x$. Assim $p_{0} \in W$ e $(W, y)$ é um sistema de coordenadas locais. Afirmamos que

$$
\frac{\partial}{\partial y_{j}}=\phi\left(\frac{\partial}{\partial \tilde{y}_{j}}\right), j=1, \ldots, N
$$

De fato,

$$
\begin{aligned}
\phi\left(\frac{\partial}{\partial \tilde{y}_{j}}\right) f & =\frac{\partial\left(f \circ x^{-1}\right)}{\partial \tilde{y}_{j}} \circ x=\frac{\partial\left(f \circ x^{-1} \circ \tilde{y}^{-1}\right)}{\partial e_{j}} \circ \tilde{y} \circ x= \\
& =\frac{\partial\left(f \circ y^{1}\right)}{\partial e_{j}} \circ y=\frac{\partial f}{\partial y_{j}}, f \in C^{\infty}(W) .
\end{aligned}
$$

Note também que

$$
\frac{\partial}{\partial \tilde{y}_{j}}=\psi\left(\frac{\partial}{\partial y_{j}}\right), j=1, \ldots, N .
$$

Resta mostrarmos que $\mathcal{V}_{p}=\left[\left(\partial / \partial y_{1}\right)_{p}, \ldots,\left(\partial / \partial y_{n}\right)_{p}\right], p \in W$. Primeiramente, vamos mostrar que $L_{j p} \in\left[\left(\partial / \partial y_{1}\right)_{p}, \ldots,\left(\partial, / \partial y_{n}\right)_{p}\right], p \in W$.

Observe que $\psi_{p}\left(L_{j p}\right)=\tilde{L}_{j q} \in\left[\left(\partial / \partial \tilde{y}_{1}\right)_{q}, \ldots,\left(\partial / \partial \tilde{y}_{n}\right)_{q}\right], p \in W, q=x(p)$, então existem $a_{j}^{k} \in \mathbb{C}, j, k=1, \ldots, n$, tais que

$$
\begin{aligned}
\psi_{p}\left(L_{j p}\right) & =\sum_{k=1}^{n} a_{j}^{k} \frac{\partial}{\partial \tilde{y}_{k q}}=\sum_{k=1}^{n} a_{j}^{k} \psi_{p}\left(\frac{\partial}{\partial y_{k}}\right)= \\
& =\psi_{p}\left(\sum_{k=1}^{n} a_{j}^{k} \frac{\partial}{\partial y_{k}}\right) .
\end{aligned}
$$

Assim

$$
L_{j p}=\sum_{k=1}^{n} a_{j}^{k} \frac{\partial}{\partial y_{k} p} \in\left[\frac{\partial}{\partial y_{1}}, \ldots, \frac{\partial}{\partial y_{n}}\right],
$$

por fim concluímos que $\mathcal{V}_{p}=\left[\left(\partial / \partial y_{1}\right)_{p}, \ldots,\left(\partial / \partial y_{n}\right)_{p}\right], p \in W$. 



\section{Estruturas Localmente Integráveis}

No presente capítulo apresentaremos o conceito de estruturas localmente integráveis e alguns resultados que nos auxiliarão no enunciado e demonstração do Teorema de Baouendi-Treves.

\subsection{Conjunto Característico}

Definição 6.1.1. Seja $\mathcal{V} \subset \mathbb{C} T \Omega$ uma estrutura involutiva. Definimos seu conjunto característico como sendo:

$$
T^{0} \doteq \cup_{p \in \Omega} T_{p}^{0}
$$

sendo que $T_{p}^{0} \doteq \mathcal{V}_{p}^{\perp} \cap T_{p}^{*} \Omega$. Assim $T^{0}=\cup_{p \in \Omega} \mathcal{V}_{p}^{\perp} \cap T_{p}^{*} \Omega=T^{\prime} \cap T^{*} \Omega$. Observe que $T_{p}^{0}$ é subespaço vetorial real de $T_{p}^{*} \Omega$.

Definição 6.1.2 (Símbolo de um campo vetorial). Seja $L \in \mathfrak{X}(\Omega)$, definimos o seu símbolo como sendo

$$
\sigma(L): T^{*} \Omega \rightarrow \mathbb{C}
$$

em que, para cada $p \in \Omega$ e $\xi \in T_{p}^{*} \Omega$, tem-se $\sigma(L)(\xi) \doteq \xi\left(L_{p}\right)$.

Observação 6.1.3. Note que $\xi \in T_{p}^{0}$ se, e somente se, $\sigma(L)(\xi)=0$ para qualquer seção $L$ de $\mathcal{V}$.

Seja uma carta local $(U, x)$ em $\Omega \operatorname{com} x=\left(x_{1}, \ldots, x_{N}\right)$ e fixe $p \in \Omega$. Para $\xi \in T_{p}^{*} \Omega$ e $L \in \mathfrak{X}(\Omega)$, tem-se $\xi=\sum_{j=1}^{N} \xi_{j} d x_{j p}, \xi_{j} \in \mathbb{R}$ e $L=\sum_{j=1}^{N} a_{j}\left(\partial / \partial x_{j}\right)$, então

$$
\sigma(L)(\xi)=\sum_{j=1}^{N} a_{j}(p) \xi_{j}
$$

Dessa forma, se $L_{j}=\sum_{k=1}^{N} a_{j k}\left(\partial / \partial x_{k}\right), j=1, \ldots, n$ são seções de $\mathcal{V}$ linearmente independentes sobre $U$ podemos descrever o conjunto $T^{0} \cap T^{*} U$ da seguinte forma

$$
\xi=\left(\xi_{1}, \ldots, \xi_{N}\right) \in T_{p}^{0} \cap T_{p}^{*} U \Leftrightarrow \sum_{k=1}^{N} a_{j k}(p) \xi_{k}=0, p \in U, \xi_{k} \in \mathbb{R}, j=1, \ldots, n .
$$

Observação 6.1.4. Seja $\Omega \subset \mathbb{R}^{N}$ aberto não vazio. Dado $L \in \mathfrak{X}(\Omega), L_{p} \neq 0, \forall p \in \Omega$, tem-se que

$$
L=\sum_{j=1}^{N} a_{j} \frac{\partial}{\partial x_{j}}, a_{j} \in C^{\infty}(\Omega), j=1, \ldots, N
$$


Este campo define também um operador diferencial parcial linear de ordem 1, a saber,

$$
P(x, D)=\sum_{j=1}^{N} a_{j}(x) \frac{\partial}{\partial x_{j}}
$$

O símbolo deste operador é dado por

$$
P_{1}(x, \xi)=\sum_{j=1}^{N} a_{j}(x) \xi_{j}, \xi \in \mathbb{R}^{N}
$$

Dizemos que P é elítico se

$$
P_{1}\left(x_{0}, \xi\right)=\sum_{j=1}^{N} a_{j}\left(x_{0}\right) \xi_{j}=0 \Leftrightarrow \xi_{j}=0, j=1, \ldots, N, \forall x_{0} \in \Omega
$$

Note que se $\mathcal{V}=\cup_{p \in \Omega} \mathcal{V}_{p}$, onde $\mathcal{V}_{p}=\left[L_{p}\right], p \in \Omega$. Então $\mathcal{V}$ é um estrutura elítica se $T_{p}^{0}=\{0\}, \forall p \in \Omega$. Em outras palavras, $\mathcal{V}$ é elítica se

$$
\sum_{j=1}^{N} a_{j}(p) \xi_{j}=0 \Leftrightarrow \xi_{j}=0, j=1, \ldots, N
$$

para cada $p \in \Omega$. Logo as definições de $\mathcal{V}$ ser estrutura chamada de elítica, que definiremos mais adiante, e $L$ ser operador elítico são equivalentes quando $\Omega \subset \mathbb{R}^{N}$ é um aberto não vazio.

Lema 6.1.5. Todo subfibrado vetorial complexo $\mathcal{V} \subset \mathbb{C} T \Omega 1$-dimensional é uma estrutura involutiva.

Demonstração. De fato, considere $\mathcal{V} \subset \mathbb{C} T \Omega$ um subfibrado vetorial complexo unidimensional. Sejam $U \subset \Omega$ for aberto e $M_{1}, M_{2} \in \mathfrak{X}(U)$ seções de $\mathcal{V}$ sobre $U$. Fixe $p_{0} \in U$, vamos mostrar que $\left[M_{1}, M_{2}\right]_{p_{0}} \in \mathcal{V}_{p_{0}}$, como $p_{0} \in U$ é arbitrário teremos que $\left[M_{1}, M_{2}\right]_{p} \in \mathcal{V}_{p}, \forall p \in U$, o resultado segue.

Diminuindo a vizinhança $U$ se necessário, existe $L \in \mathfrak{X}(U)$ tal que $\mathcal{V}_{p}=\left[L_{p}\right], \forall p \in$ $U$. Seja $x \doteq\left(x_{1}, \ldots, x_{N}\right)$ um sistema de coordenadas locais. Assim existem funções $A_{j}, a_{1 j}$, $a_{2 j} \in C^{\infty}(U), j=1, \ldots, N$, tais que

$$
\begin{aligned}
L & =\sum_{j=1}^{N} A_{j} \frac{\partial}{\partial x_{j}}, \\
M_{1} & =\sum_{k=1}^{N} a_{1 k} \frac{\partial}{\partial x_{k}}, \\
M_{2} & =\sum_{k=1}^{N} a_{2 k} \frac{\partial}{\partial x_{k}}
\end{aligned}
$$


e

$$
\begin{aligned}
L_{p} & =\sum_{j=1}^{N} A_{j}(p) \frac{\partial}{\partial x_{j}}, \\
M_{1 p} & =\sum_{k=1}^{N} a_{1 k}(p) \frac{\partial}{\partial x_{k}} \in \mathcal{V}_{p}, \\
M_{2 p} & =\sum_{k=1}^{N} a_{2 k} \frac{\partial}{\partial x_{k}} \in \mathcal{V}_{p},
\end{aligned}
$$

$p \in U$.

Logo existem $\alpha_{p}, \beta_{p} \in \mathbb{C}$ tais que $M_{1 p}=\alpha_{p} L_{p}, M_{2 p}=\beta_{p} L_{p}$. Em outras palavras,

$$
\begin{aligned}
\sum_{j=1}^{N} a_{1 j}(p) \frac{\partial}{\partial x_{j}} & =\sum_{j=1}^{N} \alpha_{p} A_{j}(p) \frac{\partial}{\partial x_{j}} \\
\sum_{j=1}^{N} a_{2 j}(p) \frac{\partial}{\partial x_{j}} & =\sum_{j=1}^{N} \beta_{p} A_{j}(p) \frac{\partial}{\partial x_{j_{p}}} .
\end{aligned}
$$

Além disso,

$$
\begin{aligned}
M_{1}\left(M_{2}\right) & =\sum_{j=1}^{N} a_{1 j} \frac{\partial}{\partial x_{j}}\left(\sum_{k=1}^{N} a_{2 k} \frac{\partial}{\partial x_{k}}\right)= \\
& =\sum_{j, k=1}^{N} a_{1 j}\left(\frac{\partial a_{2 k}}{\partial x_{j}} \frac{\partial}{\partial x_{k}}+a_{2 k} \frac{\partial^{2}}{\partial x_{j} \partial x_{k}}\right)
\end{aligned}
$$

analogamente

$$
M_{2}\left(M_{1}\right)=\sum_{j, k=1}^{N} a_{2 j}\left(\frac{\partial a_{1 k}}{\partial x_{j}} \frac{\partial}{\partial x_{k}}+a_{1 k} \frac{\partial^{2}}{\partial x_{j} \partial x_{k}}\right)
$$

e então

$$
\begin{gathered}
{\left[M_{1}, M_{2}\right]=\sum_{k=1}^{N}\left(\sum_{j=1}^{N} a_{1 j} \frac{\partial a_{2 k}}{\partial x_{j}}-a_{2 j} \frac{\partial a_{1 k}}{\partial x_{j}}\right) \frac{\partial}{\partial x_{k}}} \\
{\left[M_{1}, M_{2}\right]_{p_{0}}=\sum_{k=1}^{N}\left(\sum_{j=1}^{N} \alpha_{p_{0}} A_{j}\left(p_{0}\right) \frac{\partial a_{2 k}}{\partial x_{j}}\left(p_{0}\right)-\beta_{p_{0}} A_{j}\left(p_{0}\right) \frac{\partial a_{1 k}}{\partial x_{j}}\left(p_{0}\right)\right) \frac{\partial}{\partial x_{k}} .}
\end{gathered}
$$

Temos dois casos a considerar, o primeiro em que $M_{1 p_{0}} \neq 0$ e $M_{2 p_{0}} \neq 0$, e o segundo em que $M_{1 p_{0}}=0$ ou $M_{2 p_{0}}=0$.

Dessa forma, suponha $M_{1 p_{0}} \neq 0$ e $M_{2 p_{0}} \neq 0$ e assim existem $j_{0}, k_{0} \in\{1, \ldots, N\}$ tais que $a_{1 j_{0}}\left(p_{0}\right) \neq 0$ e $a_{2 k_{0}}\left(p_{0}\right) \neq 0$. Como $\alpha_{p_{0}} A_{j_{0}}\left(p_{0}\right)=a_{1 j_{0}}\left(p_{0}\right) \neq 0$ e $\beta_{p_{0}} A_{k_{0}}\left(p_{0}\right)=$ $a_{2 k_{0}}\left(p_{0}\right) \neq 0$, então $\alpha_{p_{0}}, \beta_{p_{0}}, A_{j_{0}}\left(p_{0}\right)$ e $A_{k_{0}}\left(p_{0}\right)$ são todos não nulos.

Diminuindo a vizinhança $U$ se necessário, obtemos que $A_{j_{0}}(p)$ e $A_{k_{0}}(p)$ são não nulos em $U$ e

$$
\begin{aligned}
& \alpha_{p}=\left(\frac{a_{1 j_{0}}}{A_{j_{0}}}\right)(p), p \in U \\
& \beta_{p}=\left(\frac{a_{2 k_{0}}}{A_{k_{0}}}\right)(p), p \in U,
\end{aligned}
$$


$\operatorname{assim} \alpha(p) \doteq \alpha_{p}$ e $\beta(p) \doteq \beta_{p}$ são funções suaves. Assim

$$
\begin{gathered}
\frac{\partial a_{1 k}}{\partial x_{j}}\left(p_{0}\right)=\frac{\partial \alpha}{\partial x_{j}}\left(p_{0}\right) A_{k}\left(p_{0}\right)+\alpha\left(p_{0}\right) \frac{\partial A_{k}}{\partial x_{j}}\left(p_{0}\right) \\
\frac{\partial a_{2 k}}{\partial x_{j}}\left(p_{0}\right)=\frac{\partial \beta}{\partial x_{j}}\left(p_{0}\right) A_{k}\left(p_{0}\right)+\beta\left(p_{0}\right) \frac{\partial A_{k}}{\partial x_{j}}\left(p_{0}\right) .
\end{gathered}
$$

Dessa forma,

$$
\begin{aligned}
{\left[M_{1}, M_{2}\right]_{p_{0}} } & =\sum_{k=1}^{N}\left(\sum_{j=1}^{N} \alpha\left(p_{0}\right) A_{j}\left(p_{0}\right) \frac{\partial \beta}{\partial x_{j}}\left(p_{0}\right)\right) A_{k}\left(p_{0}\right) \frac{\partial}{\partial x_{k} p_{0}}+ \\
& +\sum_{j, k=1}^{N} \alpha\left(p_{0}\right) A_{j}\left(p_{0}\right) \beta\left(p_{0}\right) \frac{\partial A_{k}}{\partial x_{j}}\left(p_{0}\right) \frac{\partial}{\partial x_{k}}+ \\
& +\sum_{k=1}^{N}\left(\sum_{j=1}^{N}-\beta\left(p_{0}\right) A_{j}\left(p_{0}\right) \frac{\partial \alpha}{\partial x_{j}}\left(p_{0}\right)\right) A_{k}\left(p_{0}\right) \frac{\partial}{\partial x_{k}}+ \\
& +\sum_{j, k=1}^{N}-\beta\left(p_{0}\right) A_{j}\left(p_{0}\right) \alpha\left(p_{0}\right) \frac{\partial A_{k}}{\partial x_{j}}\left(p_{0}\right) \frac{\partial}{\partial x_{k}}= \\
& =C \sum_{k=1}^{N} A_{k}\left(p_{0}\right){\frac{\partial}{\partial x_{k}}}_{p_{0}}= \\
& =C L_{p_{0}} \in \mathcal{V}_{p_{0}},
\end{aligned}
$$

em que

$$
C=\sum_{j=1}^{N}\left(\alpha\left(p_{0}\right) A_{j}\left(p_{0}\right) \frac{\partial \beta}{\partial x_{j}}\left(p_{0}\right)-\beta\left(p_{0}\right) A_{j}\left(p_{0}\right) \frac{\partial \alpha_{k}}{\partial x_{j}}\left(p_{0}\right)\right)
$$

Suponha agora que $M_{1 p_{0}}=0$. Como $\alpha_{p_{0}} L_{p_{0}}=M_{1 p_{0}}=0$, temos que $\alpha_{p_{0}}=0$. Assim

$$
\begin{aligned}
{\left[M_{1}, M_{2}\right]_{p_{0}} } & =\sum_{k, j=1}^{N}-a_{2 j}\left(p_{0}\right) \frac{\partial a_{1 k}}{\partial x_{j}}\left(p_{0}\right) \frac{\partial}{\partial x_{k}}= \\
& =-\sum_{k=1}^{N}\left(\sum_{j=1}^{N} \beta_{p_{0}} A_{j}\left(p_{0}\right) \frac{\partial a_{1 k}}{\partial x_{j}}\left(p_{0}\right)\right) \frac{\partial}{\partial x_{k}} .
\end{aligned}
$$

Como $L_{p_{0}} \neq 0$, existe $k_{0} \in\{1, \ldots, N\}$ tal que $A_{k_{0}}\left(p_{0}\right) \neq 0$. Então diminuindo a vizinhança $U$ se necessário obtemos $A_{k_{0}}(p) \neq 0, \forall p \in U$, e

$$
\alpha(p) \doteq \alpha_{p}=\frac{a_{1 k_{0}}}{A_{k_{0}}}(p), p \in U
$$

Além disso, tem-se que

$$
\frac{a_{1 k}}{\partial x_{j}}(p)=\frac{\partial \alpha}{\partial x_{j}}(p) A_{k}(p)+\frac{\partial A_{k}}{\partial x_{j}}(p) \alpha(p)
$$

e

$$
\frac{a_{1 k}}{\partial x_{j}}\left(p_{0}\right)=\frac{\partial \alpha}{\partial x_{j}}\left(p_{0}\right) A_{k}\left(p_{0}\right) .
$$


Por fim,

$$
\begin{aligned}
{\left[M_{1}, M_{2}\right]_{p_{0}} } & =\sum_{k=1}^{N}\left(-\sum_{j=1}^{N} \beta\left(p_{0}\right) A_{j}\left(p_{0}\right) \frac{\partial \alpha}{\partial x_{j}}\left(p_{0}\right)\right) A_{k}\left(p_{0}\right) \frac{\partial}{\partial x_{k} p_{0}}= \\
& =C L_{p_{0}} \in \mathcal{V}_{p_{0}},
\end{aligned}
$$

sendo que

$$
C=-\sum_{j=1}^{N} \beta\left(p_{0}\right) A_{j}\left(p_{0}\right) \frac{\partial \alpha}{\partial x_{j}}\left(p_{0}\right) .
$$

\subsection{Algumas estruturas especiais}

Seja $\mathcal{V}$ um subfibrado vetorial complexo $n$-dimensional de $\mathbb{C} T \Omega$. Dizemos que:

1. $\mathcal{V}$ define uma estrutura elítica se $T_{p}^{0}=\{0\}$, para todo $p \in \Omega$;

2. $\mathcal{V}$ define uma estrutura complexa se $\mathbb{C} T_{p} \Omega=\mathcal{V}_{p} \oplus \overline{\mathcal{V}}_{p}$, para todo $p \in \Omega$;

3. $\mathcal{V}$ define uma estrutura CR (ou de Cauchy-Riemann) se $\mathcal{V}_{p} \cap \overline{\mathcal{V}}_{p}=\{0\}$, para todo $p \in \Omega$;

4. $\mathcal{V}$ define uma estrutura essencialmente real se $\mathcal{V}_{p}=\overline{\mathcal{V}}_{p}$, para todo $p \in \Omega$.

Lema 6.2.1. Se $L_{1}, \ldots, L_{n} \in \mathfrak{X}(U)$ forem tais que $\left\{L_{1 p}, \ldots, L_{n p}\right\}$ é linearmente independente, para algum $p \in U$, então existe $V \subset U$ vizinhança de $p$ tal que $\left\{L_{1 q}, \ldots, L_{n q}\right\}$ é linearmente independente, $\forall q \in V$.

Demonstração. Sejam $p_{0} \in U \subset \Omega$ e $L_{1}, \ldots, L_{n} \in \mathfrak{X}(U)$ tais que $\left\{L_{j p_{0}}\right\}_{j=1}^{n}$ é linearmente independente. Considere $x \doteq\left(x_{1}, \ldots, x_{N}\right)$ um sistema de coordenadas locais. Assim, para cada $j=1, \ldots, n$ e $k=1, \ldots, N$, existe $a_{j k} \in C^{\infty}(U)$ com

$$
L_{j}=\sum_{k=1}^{N} a_{j k} \frac{\partial}{\partial x_{k}}, j=1, \ldots, n \text {. }
$$

Como $\left\{L_{j p_{0}}\right\}_{j=1}^{n}$ é LI, então a matriz:

$$
\left(\begin{array}{cccc}
a_{11}\left(p_{0}\right) & a_{21}\left(p_{0}\right) & \cdots & a_{n 1}\left(p_{0}\right) \\
a_{12}\left(p_{0}\right) & a_{22}\left(p_{0}\right) & \cdots & a_{n 2}\left(p_{0}\right) \\
\vdots & \vdots & \ddots & \vdots \\
a_{1 N}\left(p_{0}\right) & a_{2 N}\left(p_{0}\right) & \cdots & a_{n N}\left(p_{0}\right)
\end{array}\right)
$$


tem posto $n$. Logo, rearranjando os índices se necessário, obtém-se que det $A\left(p_{0}\right) \neq 0$, em que $A: U \rightarrow M_{n}(\mathbb{C})$ é dada por $A(p)=$

$$
\left(\begin{array}{cccc}
a_{11}(p) & a_{21}(p) & \cdots & a_{n 1}(p) \\
a_{12}(p) & a_{22}(p) & \cdots & a_{n 2}(p) \\
\vdots & \vdots & \ddots & \vdots \\
a_{1 n}(p) & a_{2 n}(p) & \cdots & a_{n n}(p)
\end{array}\right)
$$

Como cada $a_{j k}$ é suave e det é contínua, então deto $A$ é contínua, sendo assim existe aberto $V \subset U$ que contém $p_{0}$ tal que $\operatorname{det} A(p) \neq 0, \forall p \in V$.

Considere a aplicação $B: V \rightarrow M_{n}(\mathbb{C})$ dada por $B(p)=$

$$
\left(\begin{array}{cccc}
a_{1 n+1}(p) & a_{2 n+1}(p) & \cdots & a_{n n+1}(p) \\
a_{1 n+2}(p) & a_{2 n+2}(p) & \cdots & a_{n n+2}(p) \\
\vdots & \vdots & \ddots & \vdots \\
a_{1 N}(p) & a_{2 N}(p) & \cdots & a_{n N}(p) .
\end{array}\right)
$$

Assim a matriz

$$
\left(\begin{array}{c}
A(p) \\
B(p)
\end{array}\right)
$$

tem posto $n$ para todo $p \in V$ e tal matriz representa os coeficientes dos vetores $L_{1 p}, \ldots, L_{n p}$ na base canônica, a saber $\left\{\left(\partial / \partial x_{1}\right)_{p}, \ldots,\left(\partial / \partial x_{N}\right)_{p}\right\}$, portanto $\left\{L_{1 p}, \ldots, L_{n p}\right\}$ é linearmente independente para todo $p \in V$.

Proposição 6.2.2. Toda estrutura essencialmente real é localmente gerada por campos vetoriais reais.

Demonstração. Sejam $p_{0} \in U \subset \Omega$ e $L_{1}, \ldots, L_{n} \in \mathfrak{X}(U)$ tais que

$$
\mathcal{V}_{p}=\left[L_{1 p}, \ldots, L_{n p}\right], \forall p \in U
$$

Note que, $L_{j}=\Re L_{j}+i \Im L_{j}, j=1, \ldots, n$, com $\Re L_{j}, \Im L_{j}$ campos reais. Assim

$$
L_{j p}=\Re L_{j p}+i \Im L_{j p}, j=1, \ldots, n, p \in U,
$$

ou seja,

$$
\begin{aligned}
L_{j p} & \in\left[\Re L_{1 p}, \Im L_{1 p}, \ldots, \Re L_{n p}, \Im L_{n p}\right], j=1, \ldots, n, p \in U, \Rightarrow \\
\Rightarrow & \mathcal{V}_{p}=\left[L_{1 p}, \ldots, L_{n p}\right] \subset\left[\Re L_{1 p}, \Im L_{1 p}, \ldots, \Re L_{n p}, \Im L_{n p}\right], p \in U .
\end{aligned}
$$

Por outro lado, como $\mathcal{V}$ é esssencialmente real tem-se que $\mathcal{V}_{p}=\overline{\mathcal{V}}_{p}, \forall p \in \Omega$, e assim $\overline{L_{j p}} \in \overline{\mathcal{V}_{p}}=\mathcal{V}_{p}, \forall p \in U$. Consequentemente

$$
\Re L_{j p}=\frac{L_{j p}+\overline{L_{j p}}}{2}, \Im L_{j p}=\frac{L_{j p}-\overline{L_{j p}}}{2 i} \in \mathcal{V}_{p}, j=1, \ldots, n, p \in U,
$$


ou seja, $\Re L_{j}$, $L_{j}$ são seções de $\mathcal{V}$ sobre $U$. Segue que $\mathcal{V}_{p}=\left[\Re L_{1 p}, \Im L_{1 p}, \ldots, \Re L_{n p}, \Im L_{n p}\right]$, $p \in U$.

Por fim, existem $M_{1}, \ldots, M_{n} \in\left\{\Re L_{j}, \Im L_{j} ; j=1, \ldots, n\right\}$ tais que

$$
\mathcal{V}_{p_{0}}=\left[M_{1 p_{0}}, \ldots, M_{n p_{0}}\right]
$$

ou seja, $M_{1 p}, \ldots, M_{n p} \subset \mathcal{V}_{p}, p \in U$, são campos reais tais que $\left\{M_{j p_{0}}\right\}_{j=1}^{n}$ é LI, pelo Lema 6.2.1, existe aberto $V \subset U$ que contém $p_{0}$ tal que $\left\{M_{j p}\right\}_{j=1}^{n} \subset \mathcal{V}_{p}$ é LI para todo $p \in V$ e concluímos que

$$
\mathcal{V}_{p}=\left[M_{1 p}, \ldots, M_{n p}\right], p \in U
$$

Lema 6.2.3. Se $V$ é um subespaço vetorial de $\mathbb{C}^{N}=\mathbb{R}^{N}+i \mathbb{R}^{N}$ e se $V^{0} \doteq V \cap \mathbb{R}^{N}$, então $V^{0}+i V^{0}=V \cap \bar{V}$.

Demonstração. Seja $z \in V^{0}+i V^{0}$. Assim $z=x+i y$ onde

$$
\begin{aligned}
& x \doteq\left(x_{1}, \ldots, x_{N}\right) \in V^{0}=V \cap \mathbb{R}^{N} \\
& y \doteq\left(y_{1}, \ldots, y_{N}\right) \in V^{0}=V \cap \mathbb{R}^{N} .
\end{aligned}
$$

Então, obtemos que $z \in V$ e

$$
\bar{z}=\bar{x}-i \bar{y}=x-i y \in V
$$

ou seja, $z=\overline{\bar{z}} \in \bar{V}$. Concluímos que $V^{0}+i V^{0} \subset V \cap \bar{V}$.

Por outro lado, se $z \in V \cap \bar{V}$ então

$$
\begin{array}{r}
z \doteq\left(z_{1}, \ldots, z_{N}\right) \in \mathbb{C}^{N} \\
\bar{z}=\left(\overline{z_{1}}, \ldots, \overline{z_{N}}\right) \in V .
\end{array}
$$

Além disso,

$$
z=\frac{z+\bar{z}}{2}-i \frac{(i z-i \bar{z})}{2} \in V^{0}+i V^{0}
$$

pois

$$
\begin{aligned}
\frac{z+\bar{z}}{2} & \in V \cap \mathbb{R}^{N}=V^{0}, \\
\frac{(i z-i \bar{z})}{2} & \in V \cap \mathbb{R}^{N}=V^{0}
\end{aligned}
$$

Agora, utilizando o Lema 6.2.3, obtemos o seguinte resultado: 
Observação 6.2.4. Sejam $X$ um espaço vetorial complexo de dimensão $N$ e $\left\{x_{1}, \ldots, x_{N}\right\}$ uma base para tal espaço. Dessa maneira, existe isomorfismo

$$
\begin{aligned}
T: X & \rightarrow \mathbb{C}^{N} \\
x=\sum_{j=1}^{N} \alpha_{j} x_{j} & \mapsto T x \doteq\left(\alpha_{1}, \ldots, \alpha_{N}\right) .
\end{aligned}
$$

Sabemos que $\mathbb{C}^{N}=\mathbb{R}^{N}+i \mathbb{R}^{N}$. Considere $E \doteq T^{-1}\left(\mathbb{R}^{N}\right) \subset X$. Observe que $X=$ $E+i E$, pois $X=T^{-1}\left(\mathbb{C}^{N}\right)=T^{-1}\left(\mathbb{R}^{N}+i \mathbb{R}^{N}\right)=T^{-1}\left(\mathbb{R}^{N}\right)+i T^{-1}\left(\mathbb{R}^{N}\right)=E+i E$.

Se $x \in X$, definimos $\bar{x} \doteq T^{-1}(\overline{T x})$ e se $V \subset X$ definimos

$$
\bar{V}=\{\bar{x} ; x \in V\} \text {. }
$$

Note que se $x \in X$ então

$$
x=\sum_{j=1}^{N} \alpha_{j} x_{j}, \alpha_{j} \in \mathbb{C},
$$

e assim

$$
\bar{x}=T^{-1}(\overline{T x})=T^{-1}\left(\overline{\alpha_{1}}, \ldots, \overline{\alpha_{N}}\right)=\sum_{j=1}^{N} \overline{\alpha_{j}} x_{j} .
$$

Afirmamos que se $V \subset X$ e $V^{0} \doteq V \cap E$, então $V^{0}+i V^{0}=V \cap \bar{V}$. Com efeito, seja $V \subset X$ e considere $V^{0} \doteq V \cap E$. Então $\tilde{V} \doteq T(V) \subset \mathbb{C}^{N}$ é subespaço vetorial $e$ $\tilde{V}^{0} \doteq T(V) \cap T(E)=\tilde{V} \cap \mathbb{R}^{N}$.

Dessa forma, $\tilde{V}^{0}+i \tilde{V}^{0}=\tilde{V} \cap \bar{V}$, como

$$
\begin{aligned}
\overline{\tilde{V}} & =\overline{T(V)}=\{\overline{T x} ; x \in V\}, \\
T^{-1}(\overline{\tilde{V}}) & =\left\{T^{-1}(\overline{T x}) ; x \in V\right\}=\{\bar{x} ; x \in V\}=\bar{V},
\end{aligned}
$$

obtemos que

$$
T\left(V^{0}+i V^{0}\right)=T\left(V^{0}\right)+i T\left(V^{0}\right)=\tilde{V}^{0}+i \tilde{V}^{0}=\tilde{V} \cap \overline{\tilde{V}}=T(V) \cap \overline{T(V)} .
$$

Portanto

$$
V^{0}+i V^{0}=T^{-1}\left(T\left(V^{0}+i V^{0}\right)\right)=T^{-1}(T(V) \cap \overline{T(V)})=V \cap T^{-1}(\overline{T(V)})=V \cap \bar{V} .
$$

Observação 6.2.5. Observe que $\mathbb{C} T_{p} \Omega$ e $\mathbb{C} T_{p}^{*} \Omega$ são espaços vetoriais $N$-dimensionais, $T_{p} \Omega=T^{-1}\left(\mathbb{R}^{N}\right)$ e $T_{p}^{*} \Omega=T^{-1}\left(\mathbb{R}^{N}\right)$. Assim

$$
\begin{aligned}
\mathbb{C} T_{p} \Omega & =T_{p} \Omega+i T_{p} \Omega, \\
\mathbb{C} T_{p}^{*} \Omega & =T_{p}^{*} \Omega+i T_{p}^{*} \Omega .
\end{aligned}
$$

Seja $\mathcal{V}$ uma estrutura involutiva $n$-dimensional sobre $\Omega$. Daí, tem-se que, para cada $p \in \Omega, V \doteq \mathcal{V}_{p}^{\perp} \subset \mathbb{C}_{p}^{*} \Omega$ é subespaço vetorial $m$-dimensional e $V^{0}=V \cap E=$ $\mathcal{V}_{p}^{\perp} \cap T_{p}^{*} \Omega=T_{p}^{0}$. Por fim, concluímos que

$$
T_{p}^{0}+i T_{p}^{0}=\mathcal{V}_{p}^{\perp} \cap \overline{\mathcal{V}_{p}^{\perp}}
$$


Corolário 6.2.6. Toda estrutura complexa é uma estrutura elítica.

Demonstração. Seja $\mathcal{V}$ uma estrutura complexa, ou seja, $\mathbb{C} T_{p} \Omega=\mathcal{V}_{p} \oplus \overline{\mathcal{V}}_{p}, \forall p \in \Omega$, e vamos mostrar que $T_{p}^{0}=\{0\}, \forall p \in \Omega$.

Primeiramente, note que se $\mathcal{V}_{p}=\left[v_{1}, \ldots, v_{n}\right]$ então $\overline{\mathcal{V}}_{p}=\left[\overline{v_{1}}, \ldots, \overline{v_{n}}\right]$ e $\mathcal{V}_{p} \cap \overline{\mathcal{V}}_{p}=$ $\{0\}$. Assim $\mathbb{C} T_{p} \Omega=\left[v_{1}, \ldots, v_{n}, \bar{v}_{1}, \ldots, \bar{v}_{n}\right]$ e $N=\operatorname{dim} \mathbb{C} T_{p} \Omega=2 n$.

Seja $\left\{\eta_{j}\right\}_{j=1}^{2 n}$ base de $\mathbb{C} T_{p}^{*} \Omega$ dual de $\left\{v_{1}, \ldots, v_{n}, \bar{v}_{1}, \ldots, \bar{v}_{n}\right\}$, ou seja,

$$
\begin{aligned}
\eta_{j}\left(v_{k}\right) & =\delta_{j k}, j, k=1, \ldots, n, \\
\eta_{j}\left(\overline{v_{k}}\right) & =0, j, k=1, \ldots, n, \\
\eta_{j+n}\left(\overline{v_{k}}\right) & =\delta_{j k}, j, k=1, \ldots, n, \\
\eta_{j+n}\left(v_{k}\right) & =0, j, k=1, \ldots, n .
\end{aligned}
$$

Observe que

$$
\begin{aligned}
& \bar{\eta}_{j}\left(v_{k}\right)=\overline{\eta_{j}\left(\bar{v}_{k}\right)}=0=\eta_{j+n}\left(v_{k}\right), j, k=1, \ldots, n, \\
& \bar{\eta}_{j}\left(\bar{v}_{k}\right)=\overline{\eta_{j}\left(v_{k}\right)}=\delta_{j k}=\eta_{j+n}\left(\bar{v}_{k}\right), j, k=1, \ldots, n,
\end{aligned}
$$

ou seja, $\eta_{j+n}=\bar{\eta}_{j}, j=1, \ldots, n$.

Além disso, como $\mathcal{V}_{p}=\left[v_{1}, \ldots, v_{n}\right]$ então

$$
\eta \in \mathcal{V}_{p}^{\perp} \Leftrightarrow \eta\left(v_{k}\right)=0, k=1, \ldots, n
$$

e

$$
\eta=\sum_{j=1}^{n} \eta\left(v_{j}\right) \eta_{j}+\sum_{j=1}^{n} \eta\left(\bar{v}_{j}\right) \bar{\eta}_{j}=\sum_{j=1}^{n} \eta\left(\bar{v}_{j}\right) \bar{\eta}_{j} \in\left[\eta_{1}, \ldots, \eta_{n}\right] .
$$

Logo $\mathcal{V}_{p}^{\perp}=\left[\bar{\eta}_{1}, \ldots, \bar{\eta}_{n}\right]$ e $\overline{\mathcal{V}}_{p}^{\perp}=\left[\eta_{1}, \ldots, \eta_{n}\right]$. Dessa forma, tem-se que $\mathcal{V}_{p}^{\perp} \cap \overline{\mathcal{V}}_{p}^{\perp}=\{0\}$ e $\mathbb{C} T_{p}^{*} \Omega=\mathcal{V}_{p}^{\perp} \oplus \overline{\mathcal{V}}_{p}^{\perp}$, pois $\mathbb{C} T_{p}^{*} \Omega=\left[\bar{\eta}_{1}, \ldots, \bar{\eta}_{n}, \eta_{1}, \ldots, \eta_{n}\right]$

Por fim, como $\{0\} \subset T_{p}^{0}=T_{p}^{0}+i\{0\} \subset T_{p}^{0}+i T_{p}^{0} \subset \mathcal{V}_{p}^{\perp} \cap \overline{\mathcal{V}}_{p}^{\perp}=\{0\}$, concluímos que $T_{p}^{0}=\{0\}, \forall p \in \Omega$.

Exemplo 6.2.7 (Operador de Mizohata). Seja

$$
M \doteq-t i \frac{\partial}{\partial x}+\frac{\partial}{\partial t} \in \mathfrak{X}\left(\mathbb{R}^{2}\right),
$$

em que $(x, t)$ é o sistema de coordenadas de $\mathbb{R}^{2}$, ou seja, $x\left(p_{1}, p_{2}\right)=p_{1}$ e $t\left(p_{1}, p_{2}\right)=p_{2}$ para $p=\left(p_{1}, p_{2}\right) \in \mathbb{R}^{2}$.

Observe que $M_{p} \neq 0, \forall p \in \mathbb{R}^{2}$, pois $M_{p}=-i p_{2}(\partial / \partial x)_{p}+(\partial / \partial t)_{p}$ e $M_{p}(\underline{t})=1 \neq$ $0, p \in \mathbb{R}^{2}$. Considere $\mathcal{V}=\cup_{p \in \mathbb{R}^{2}} \mathcal{V}_{p} \subset \mathbb{C T R}^{2}$, assim $\mathcal{V}$ é subfibrado vetorial complexo unidimensional e pelo Lema 6.1 .5 obtemos que tal estrutura é involutiva. 
Agora, para cada $p \in \mathbb{R}^{2}$, vamos calcular $\mathcal{V}_{p}^{\perp}$. Tem-se que

$$
\begin{aligned}
\xi \in \mathcal{V}_{p}^{\perp} & \Leftrightarrow \xi(v)=0, \forall v \in \mathcal{V}_{p}=\left[M_{p}\right] \Leftrightarrow \xi\left(M_{p}\right)=-i p_{2} \xi\left(\frac{\partial}{\partial x_{p}}\right)+\xi\left(\frac{\partial}{\partial t_{p}}\right)=0 \Leftrightarrow \\
& \Leftrightarrow \xi\left(\frac{\partial}{\partial x_{p}}\right)=i p_{2} \xi\left(\frac{\partial}{\partial t_{p}}\right)
\end{aligned}
$$

e assim

$$
\xi \in \mathcal{V}_{p}^{\perp} \Leftrightarrow \xi=\xi\left(\frac{\partial}{\partial x_{p}}\right)\left(d x_{p}+i p_{2} d t_{p}\right) .
$$

Portanto $\mathcal{V}_{p}^{\perp} \subset\left[d x_{p}+i p_{2} d t_{p}\right]$, como $\operatorname{dim} \mathcal{V}_{p}^{\perp}=N-n=2-1=1$ concluímos que $\mathcal{V}_{p}^{\perp}=\left[d x_{p}+i p_{2} d t_{p}\right]$.

Observe que para $p=\left(p_{1}, 0\right) \in \mathbb{R}^{2}$ temos $\mathcal{V}_{p}^{\perp}=\left[d x_{p}\right]$ e $d x_{p} \in T_{p}^{*} \mathbb{R}^{2}$. Como $d x_{p} \neq 0$ e $d x_{p} \in T_{p}^{0}$, obtemos que $\mathcal{V}$ não é elítica. Consequentemente, $\mathcal{V}$ não é uma estrutura complexa.

Note que $\mathcal{V}$ não é essencialmente real, caso fosse teríamos $\mathcal{V}_{p}=\overline{\mathcal{V}}_{p}, \forall p \in \mathbb{R}^{2}$. Porém, para $p=(0,1) \in \mathbb{R}^{2}$, tem-se que

$$
\begin{aligned}
M_{p} & =-i \frac{\partial}{\partial x_{p}}+\frac{\partial}{\partial t}_{p} \\
\bar{M}_{p} & =i \frac{\partial}{\partial x_{p}}+\frac{\partial}{\partial t}_{p}
\end{aligned}
$$

Se $\mathcal{V}_{p}=\overline{\mathcal{V}}_{p}$, então

$$
\begin{aligned}
\frac{\partial}{\partial t_{p}} & =\frac{M_{p}+\bar{M}_{p}}{2} \in \mathcal{V}_{p}, \\
\frac{\partial}{\partial x_{p}} & =\frac{\bar{M}_{p}-M_{p}}{2 i} \in \mathcal{V}_{p}
\end{aligned}
$$

e assim $\mathbb{C} T_{p} \mathbb{R}^{2}=\left[(\partial / \partial x)_{p},(\partial / \partial t)_{p}\right] \subset \mathcal{V}_{p}$, o que é absurdo pois dim $\mathcal{V}_{p}=1$

Além disso, $\mathcal{V}$ não é uma estrutura $C R$. Caso $\mathcal{V}$ fosse $C R$, teríamos que $\mathcal{V}_{p} \cap \overline{\mathcal{V}}_{p}=$ $\{0\}$, mas note que, para $p=\left(p_{1}, 0\right) \in \mathbb{R}^{2}$ e assim

$$
M_{p}=\frac{\partial}{\partial t}_{p}=\bar{\partial}_{\partial t_{p}}=\bar{M}_{p} \neq 0,
$$

o que implica em $M_{p} \in \mathcal{V}_{p} \cap \overline{\mathcal{V}}_{p}$ e $M_{p} \neq 0$. Logo $\mathcal{V}$ não é uma estrutura $C R$.

Por fim, vamos calcular $T_{p}^{0}, p \in \mathbb{R}^{2}$. Seja $\xi \in T_{p}^{0}=\mathcal{V}_{p}^{\perp} \cap T_{p}^{*} \mathbb{R}^{2}$ então

$$
\xi=\alpha d x_{p}+\alpha i p_{2} d t_{p}, \alpha, \alpha i p_{2} \in \mathbb{R} \text {. }
$$

Se $p=\left(p_{1}, 0\right)$, então $\xi=\alpha d x_{p}, \alpha \in \mathbb{R}$. Se $p=\left(p_{1}, p_{2}\right)$ com $p_{2} \neq 0$, então $\xi=\alpha d x_{p}+$ $\alpha i p_{2} d t_{p}, \alpha, \alpha i p_{2} \in \mathbb{R}$, e assim $\alpha=0$, ou seja, $\xi=0$. Em outras palavras, tem-se que

$$
\begin{aligned}
T_{p}^{0} & =\{0\}, p \in \mathbb{R}^{2}-(\mathbb{R} \times\{0\}), \\
T_{p}^{0} & =\left[d x_{p}\right], p \in \mathbb{R} \times\{0\} .
\end{aligned}
$$


Proposição 6.2.8. Seja $\mathcal{V}$ uma estrutura $C R$-dimensional sobre $\Omega$. Então seu conjunto característico $T^{0}$ é um subfibrado vetorial real $(N-2 n)$-dimensional de $T^{*} \Omega$.

Demonstração. Seja $\mathcal{V}$ uma estrutura $\mathrm{CR} n$-dimensional sobre $\Omega$, assim $\mathcal{V}_{p} \cap \overline{\mathcal{V}_{p}}=$ $\{0\}, \forall p \in \Omega$. Tome $\mathcal{W}_{p} \doteq \mathcal{V}_{p} \oplus \overline{\mathcal{V}_{p}}, p \in \Omega$, e $\mathcal{W} \doteq \cup_{p \in \Omega} \mathcal{W}_{p}$

Observe que $\mathcal{W} \subset \mathbb{C} T \Omega$ é subfibrado vetorial complexo $2 n$-dimensional. De fato, como $\mathcal{V}_{p} \cap \overline{\mathcal{V}_{p}}=\{0\}, \forall p \in \Omega$, então $\mathcal{W}_{p} \doteq \mathcal{V}_{p} \oplus \overline{\mathcal{V}_{p}}$ é subespaço vetorial $2 n$-dimensional de $\mathbb{C} T_{p} \Omega$.

Além disso, dado $p_{0} \in \Omega$ temos que existe $U \subset \Omega$ com $p_{0} \in U$ e $L_{1}, \ldots, L_{n} \in \mathfrak{X}(U)$ tais que

$$
\begin{aligned}
& \mathcal{V}_{p}=\left[L_{1 p}, \ldots, L_{n p}\right], p \in U, \\
& \overline{\mathcal{V}_{p}}=\left[\overline{L_{1 p}}, \ldots, \overline{L_{n p}}\right], p \in U,
\end{aligned}
$$

$\log 0$

$$
\mathcal{W}_{p}=\left[L_{1 p}, \ldots, L_{n p}, \overline{L_{1 p}}, \ldots, \overline{L_{n p}}\right], p \in U
$$

Agora vamos mostrar $\mathcal{W}$ é uma estrutura essencialmente real. Fixe $p \in \Omega$, seja $v \in \mathcal{W}_{p}$ assim

$$
v=v_{1}+v_{2}, v_{1} \in \mathcal{V}_{p}, v_{2} \in \overline{\mathcal{V}_{p}} \Rightarrow \bar{v}=\overline{v_{1}}+\overline{v_{2}}, \overline{v_{2}} \in \mathcal{V}_{p}, \overline{v_{1}} \in \overline{\mathcal{V}_{p}},
$$

então $\bar{v} \in \mathcal{W}_{p}$ e $v=\overline{\bar{v}} \in \overline{\mathcal{W}_{p}}$, ou seja, $\mathcal{W}_{p} \subset \overline{\mathcal{W}_{p}}$. Por outro lado, se $v \in \overline{\mathcal{W}_{p}}$ então $\bar{v} \in \mathcal{W}_{p}$ e

$$
\bar{v}=v_{1}+v_{2}, v_{1} \in \mathcal{V}_{p}, v_{2} \in \overline{\mathcal{V}_{p}} \Rightarrow v=\overline{v_{1}}+\overline{v_{2}}, \overline{v_{2}} \in \mathcal{V}_{p}, \overline{v_{1}} \in \overline{\mathcal{V}_{p}}
$$

$\operatorname{assim} v \in \overline{\mathcal{W}_{p}}$, ou seja, $\overline{\mathcal{W}_{p}} \subset \mathcal{W}_{p}$ e portanto $\mathcal{W}_{p}=\overline{\mathcal{W}_{p}}$

Dado $p_{0} \in \Omega$, pela Proposição 6.2.2 temos que existem $V \subset \Omega$, com $p_{0} \in V$, e $\eta_{1}, \ldots, \eta_{N-2 n} \in \mathfrak{N}(V)$ reais tais que $\mathcal{W}_{p}^{\perp}=\operatorname{span}_{\mathbb{C}}\left\{\eta_{1 p}, \ldots, \eta_{N-2 n p}\right\}, p \in V$. Assim $\eta_{j p}(v)=$ $0, v \in \mathcal{W}_{p}, j=1, \ldots, N-2 n$, e então $\eta_{j p}(v)=0, v \in \mathcal{V}_{p}, j=1, \ldots, N-2 n$, pois $\mathcal{V}_{p} \subset \mathcal{W}_{p}$.

$\operatorname{Logo} \eta_{j p} \in \mathcal{V}_{p}^{\perp} \cap T_{p}^{*} \Omega, j=1, \ldots, N-2 n$. Daí, se $\eta \in T_{p}^{0}$ tem-se que

$$
\eta=\sum_{j=1}^{N-2 n} \alpha_{j} \eta_{j p}, \alpha_{j} \in \mathbb{C},
$$

mas como $\eta \in T_{p}^{*} \Omega$ então

$$
\eta=\bar{\eta}=\sum_{j=1}^{N-2 n} \overline{\alpha_{j}} \eta_{j p},
$$

dessa forma $\eta_{j}=\overline{\eta_{j}}, j=1, \ldots, N-2 n$, ou seja, $\eta_{j} \in \mathbb{R}, j=1, \ldots, N-2 n$.

Por fim, concluímos que $T_{p}^{0}=\operatorname{span}_{\mathbb{R}}\left\{\eta_{j p} ; j=1, \ldots, N-2 n\right\}$, pois $\left\{\eta_{j p} ; j=\right.$ $1, \ldots, N-2 n\}$ é linearmente independente e geram $T_{p}^{0}$ sobre $\mathbb{R}$. 
Lema 6.2.9. Considere $V \subset \mathbb{C}^{N}$ um subespaço vetorial $m$-dimensional. Sejam $V^{0} \doteq$ $V \cap \mathbb{R}^{N}, d \doteq \operatorname{dim}_{\mathbb{R}} V^{0}, \nu \doteq m-d$. Considere também $V_{1} \subset \mathbb{C}^{N}$ um subespaço vetorial tal que $V=\left(V^{0} \oplus i V^{0}\right) \oplus V_{1}, e$ :

- $\left\{\zeta_{1}, \ldots, \zeta_{\nu}\right\}$ uma base de $V_{1}$,

- $\left\{\xi_{\nu+1}, \ldots, \xi_{m}\right\}$ uma base de $V^{0}$ como espaço vetorial real.

Se $\alpha_{j} \doteq \Re \zeta_{j}, \eta_{j} \doteq \Im \zeta_{j}, j=1, \ldots, \nu$, então:

1. $\left\{\zeta_{1}, \ldots, \zeta_{\nu}, \xi_{\nu+1}, \ldots, \xi_{m}\right\}$ é uma base de $V$;

2. $\left\{\alpha_{1}, \ldots, \alpha_{\nu}, \xi_{\nu+1}, \ldots, \xi_{m}, \eta_{1}, \ldots, \eta_{\nu}\right\}$ é linearmente independente sobre $\mathbb{R}$;

3. $\nu+m \leq N$.

Observação 6.2.10. Note que se $V_{1} \subset \mathbb{C}^{N}$ um subespaço vetorial tal que $V=\left(V^{0} \oplus\right.$ $\left.i V^{0}\right) \oplus V_{1}$, então $\operatorname{dim}_{\mathbb{C}} V_{1}=\nu$. De fato, se $v \in V^{0} \oplus i V^{0}$ então

$$
v=\sum_{j=1}^{d} a_{j} \xi_{\nu+j}+\sum_{j=1}^{d} b_{j} i \xi_{\nu+j}, a_{j}, b_{j} \in \mathbb{R} .
$$

Se $\alpha_{1}, \ldots, \alpha_{d}, \beta_{1}, \ldots, \beta_{d} \in \mathbb{R}$ forem tais que

$$
\sum_{j=1}^{d} \alpha_{j} \xi_{\nu+j}+\sum_{j=1}^{d} \beta_{j} i \xi_{\nu+j}=0
$$

então

$$
\sum_{j=1}^{d} \alpha_{j} \xi_{\nu+j}-\sum_{j=1}^{d} \beta_{j} i \xi_{\nu+j}=\overline{0}=0
$$

$\log o$

$$
\sum_{j=1}^{d} \alpha_{j} \xi_{\nu+j}=0, \sum_{j=1}^{d} \beta_{j} \xi_{\nu+j}=0 .
$$

Como $\left\{\xi_{\nu+j}\right\}_{j=1}^{d}$ é base de $V^{0}$, tem-se que $\alpha_{j}=\beta_{j}=0, j=1, \ldots, d$. Portanto $\operatorname{dim}_{\mathbb{R}}\left(V^{0} \oplus\right.$ $\left.i V^{0}\right)=2 d$, dessa forma $\operatorname{dim}_{\mathbb{C}}\left(V^{0} \oplus i V^{0}\right)=d$ e concluímos que $\operatorname{dim}_{\mathbb{C}} V_{1}=\operatorname{dim}_{\mathbb{C}} V-$ $\operatorname{dim}_{\mathbb{C}}\left(V^{0} \oplus i V^{0}\right)=m-d=\nu$.

Demonstração. Seja $v \in V$, assim $v=v_{0}+v_{1}, v_{0} \in V^{0} \oplus i V^{0}, v_{1} \in V_{1}$ e então existem $u, \tilde{u} \in V^{0}$ e $\beta_{1}, \ldots, \beta_{\nu} \in \mathbb{C}$ tais que

$$
v_{0}=u+i \tilde{u}, v_{1}=\sum_{j=1}^{\nu} \beta_{j} \zeta j .
$$

Por outro lado, existem $a_{1}, \ldots, a_{d}, b_{1}, \ldots, b_{d} \in \mathbb{R}$ tais que

$$
u=\sum_{j=1}^{d} a_{j} \xi_{\nu+j}, \tilde{u}=\sum_{j=1}^{d} b_{j} \xi_{\nu+j},
$$


dessa forma

$$
v=\sum_{j=1}^{d} c_{j} \xi_{\nu+j}+\sum_{j=1}^{\nu} \beta_{j} \zeta_{j},
$$

onde $c_{j}=a_{j}+i b_{j} \in \mathbb{C}, j=1, \ldots, d$. Assim $S \doteq\left\{\zeta_{1}, \ldots, \zeta_{\nu}, \xi_{\nu+1}, \ldots, \xi_{m}\right\}$ gera $V$. Resta mostrar que tal conjunto é linearmente independente sobre $\mathbb{C}$.

Se $a_{1}, \ldots, a_{\nu}, b_{1}, \ldots, b_{d} \in \mathbb{C}$ forem tais que

$$
\sum_{j=1}^{d} b_{j} \xi_{\nu+j}+\sum_{j=1}^{\nu} a_{j} \zeta_{j}=0
$$

ou seja,

$$
\sum_{j=1}^{d} b_{j} \xi_{\nu+j}=-\sum_{j=1}^{\nu} a_{j} \zeta_{j} \in V_{1} .
$$

Como $V_{1} \cap V^{0} \oplus i V^{0}=\{0\}$, tem-se que

$$
\sum_{j=1}^{d} b_{j} \xi_{\nu+j}=-\sum_{j=1}^{\nu} a_{j} \zeta_{j}=0
$$

assim $a_{1}=\ldots=a_{\nu}=b_{1}=\ldots=b_{d}=0$, concluindo que $S$ é linearmente independente.

Afirmamos agora que $V \cap \bar{V}_{1}=\{0\}$. Com efeito, se $z \in V \cap \bar{V}_{1}$ então

$$
z \in V \mathrm{e} \bar{z} \in V_{1} \subset V \Rightarrow z \in V \cap \bar{V}=V^{0} \oplus i V^{0}
$$

assim

$$
\begin{aligned}
z & =x+i y, x, y \in V^{0}=V \cap \mathbb{R}^{N} \Rightarrow \bar{z}=x-i y \in V^{0} \oplus i V^{0} \Rightarrow \\
& \Rightarrow \bar{z} \in V_{1} \cap\left(V^{0} \oplus i V^{0}\right)=\{0\} \Rightarrow \bar{z}=0 \Rightarrow z=0 .
\end{aligned}
$$

Considere $\tilde{S} \doteq\left\{\zeta_{1}, \ldots, \zeta_{\nu}, \overline{\zeta_{1}}, \ldots, \overline{\zeta_{\nu}}, \xi_{\nu+1}, \ldots, \xi_{m}\right\}$ e mostremos que tal conjunto é linearmente independente. Sejam $a_{1}, \ldots, a_{\nu}, b_{1}, \ldots, b_{\nu}, c_{1}, \ldots, c_{d} \in \mathbb{C}$ tais que

$$
\sum_{k=1}^{\nu} a_{k} \overline{\zeta_{k}}+\sum_{k=1}^{\nu} b_{k} \zeta_{k}+\sum_{k=1}^{\nu} c_{k} \xi_{k}=0,
$$

então

$$
\sum_{k=1}^{\nu} b_{k} \zeta_{k}+\sum_{k=1}^{\nu} c_{k} \xi_{k}=-\sum_{k=1}^{\nu} a_{k} \overline{\zeta_{k}} \in \overline{V_{1}}
$$

mas

$$
\sum_{k=1}^{\nu} b_{k} \zeta_{k}+\sum_{k=1}^{\nu} c_{k} \xi_{k} \in V \text { e } V \cap \bar{V}_{1}=\{0\}
$$

ou seja,

$$
\sum_{k=1}^{\nu} b_{k} \zeta_{k}+\sum_{k=1}^{\nu} c_{k} \xi_{k}=-\sum_{k=1}^{\nu} a_{k} \overline{\zeta_{k}}=0
$$

$\operatorname{Logo} a_{1}=\ldots=a_{\nu}=b_{1}=\ldots=b_{\nu}=c_{1}=\ldots=c_{d}=0$, pois $\left\{\zeta_{1}, \ldots, \zeta_{\nu}, \xi_{1}, \ldots, \xi_{m}\right\}$ é base de $V$ e $\left\{\overline{\zeta_{1}}, \ldots, \overline{\zeta_{\nu}}\right\}$ é base $\overline{V_{1}}$, concluindo que $\tilde{S} \subset \mathbb{C}^{N}$ é LI. Sendo assim, tem-se que

$$
\nu+m=\nu+\nu+d=\operatorname{dim}_{\mathbb{C}}[\tilde{S}] \leq \operatorname{dim}_{\mathbb{C}} \mathbb{C}^{N}=N .
$$


Por fim, vamos mostrar que $\hat{S} \doteq\left\{\alpha_{1}, \ldots, \alpha_{\nu}, \xi_{\nu+1}, \ldots, \xi_{m}, \eta_{1}, \ldots, \eta_{\nu}\right\}$ é LI sobre R. Sejam $a_{1}, \ldots, a_{\nu}, b_{1}, \ldots, b_{\nu}, c_{1}, \ldots, c_{d} \in \mathbb{R}$ tais que

$$
\sum_{k=1}^{\nu} a_{k} \alpha_{k}+\sum_{k=1}^{\nu} b_{k} \eta_{k}+\sum_{k=1}^{d} c_{k} \xi_{\nu+k}=0
$$

como $\alpha_{k}=\left(\zeta_{k}+\overline{\zeta_{k}}\right) / 2, \eta_{k}=\left(\zeta_{k}-\overline{\zeta_{k}}\right) / 2 i, k=1, \ldots, \nu$, tem-se que

$$
\sum_{k=1}^{\nu}\left(\frac{a_{k}}{2}+\frac{b_{k}}{2 i}\right) \zeta_{k}+\sum_{k=1}^{\nu}\left(\frac{a_{k}}{2}-\frac{b_{k}}{2 i}\right) \overline{\zeta_{k}}+\sum_{k=1}^{d} c_{k} \xi_{\nu+k}=0 .
$$

Além disso, por $\tilde{S}$ ser LI tem-se que

$$
\begin{aligned}
\left(\frac{a_{k}}{2}+\frac{b_{k}}{2 i}\right) & =\left(\frac{a_{k}}{2}-\frac{b_{k}}{2 i}\right)=c_{j}=0, k=1, \ldots, \nu, j=1, \ldots, d \Rightarrow \\
& \Rightarrow \quad, a_{k}=b_{k}=c_{j}=0, k=1, \ldots, \nu, j=1, \ldots, d
\end{aligned}
$$

concluindo a demonstração.

Observação 6.2.11. Sejam $X$ um espaço vetorial $N$-dimensional, $V \subset X$ um subespço vetorial $m$-dimensional, $V^{0} \doteq V \cap \mathbb{R}^{N}, d \doteq \operatorname{dim}_{\mathbb{R}} V^{0}$ e $\nu \doteq m-d$. Considere também $V_{1} \subset \mathbb{C}^{N}$ um subespaço vetorial tal que $V=\left(V^{0} \oplus i V^{0}\right) \oplus V_{1}$, e:

- $\left\{\zeta_{1}, \ldots, \zeta_{\nu}\right\}$ uma base de $V_{1}$,

- $\left\{\xi_{\nu+1}, \ldots, \xi_{m}\right\}$ uma base de $V^{0}$ como espaço vetorial real.

Se $\alpha_{j} \doteq \Re \zeta_{j}, \eta_{j} \doteq \Im \zeta_{j}, j=1, \ldots, \nu$, então:

1. $\left\{\zeta_{1}, \ldots, \zeta_{\nu}, \xi_{\nu+1}, \ldots, \xi_{m}\right\}$ é uma base de $V$;

2. $\left\{\alpha_{1}, \ldots, \alpha_{\nu}, \xi_{\nu+1}, \ldots, \xi_{m}, \eta_{1}, \ldots, \eta_{\nu}\right\}$ é linearmente independente sobre $\mathbb{R}$;

3. $\nu+m \leq N$.

De fato, tome

$$
\begin{aligned}
\tilde{V} & \doteq T(V) \subset T(X)=\mathbb{C}^{N}, \tilde{V}_{0} \doteq T\left(V_{0}\right)=T(V) \cap T(E)=\tilde{V} \cap \mathbb{R}^{N},\left(\tilde{V}_{1}\right) \doteq T\left(V_{1}\right), \\
\tilde{\zeta}_{j} & \doteq T\left(\zeta_{j}\right)=T\left(\alpha_{j}\right)+i T\left(\eta_{j}\right), j=1, \ldots, \nu \\
\tilde{\xi}_{j+\nu} & \doteq T\left(\xi_{j+\nu}\right), j=1, \ldots, d .
\end{aligned}
$$

Assim, tem-se que

$\left\{\tilde{\zeta}_{1}, \ldots, \tilde{\zeta}_{\nu}\right\} \quad$ é base de $\tilde{V}_{1}$,

$\left\{\tilde{\xi}_{\nu+1}, \ldots, \tilde{\xi}_{m}\right\} \quad$ é base real de $\tilde{V}_{0}$, 
aplicando o Lema acima obtemos que

$$
\begin{aligned}
&\left\{\tilde{\zeta}_{1}, \ldots, \tilde{\zeta}_{\nu}, \tilde{\xi}_{\nu+1}, \ldots, \tilde{\xi}_{m}\right\} \text { é base de } \tilde{V}, \\
&\left\{\tilde{\alpha}_{1}, \ldots, \tilde{\alpha}_{\nu}, \tilde{\xi}_{\nu+1}, \ldots, \tilde{\xi}_{m}, \tilde{\eta}_{1}, \ldots, \tilde{\eta}_{\nu}\right\} \text { é LI sobre } \mathbb{R}, \\
& \nu+m \leq N,
\end{aligned}
$$

onde $\tilde{\alpha}_{j}=T\left(\alpha_{j}\right), \tilde{\eta}_{j}=T\left(\eta_{j}\right), j=1, \ldots, \nu$, como $T$ é isomorfismo temos que

$$
\begin{aligned}
\left\{\zeta_{1}, \ldots, \zeta_{\nu}, \xi_{\nu+1}, \ldots, \xi_{m}\right\} & \text { é base de } V, \\
\left\{\alpha_{1}, \ldots, \alpha_{\nu}, \xi_{\nu+1}, \ldots, \xi_{m}, \eta_{1}, \ldots, \eta_{\nu}\right\} & \text { é LI sobre } \mathbb{R} .
\end{aligned}
$$

\subsection{Estruturas Localmente Integráveis}

Definição 6.3.1 (Estruturas Localmente Integráveis). Seja $\mathcal{V} \subset \mathbb{C} T \Omega$ um subfibrado complexo $n$-dimensional. Dizemos que $\mathcal{V}$ define uma estrutura localmente integrável sobre $\Omega$ se, dado $p_{0} \in \Omega$, existem $U_{0} \subset \Omega$ aberto, com $p_{0} \in U_{0}$, e $Z_{1}, \ldots, Z_{m} \in C^{\infty}\left(U_{0}\right)$ tal que

$$
\mathcal{V}_{p}^{\perp}=\left[d Z_{1 p}, \ldots, d Z_{m p}\right], p \in U_{0}
$$

onde $m \doteq N-n$.

É importante ressaltar que toda estrutura localmente integrável $\mathcal{V} \subset \mathbb{C} T \Omega$ é uma estrutura involutiva. Para demonstrar tal fato faremos uso do seguinte lema:

Lema 6.3.2. Sejam $X$ um espaço vetorial sobre $\mathbb{C}$ e $\phi, \phi_{1}, \ldots, \phi_{n} \in: X \rightarrow \mathbb{C}$ operadores lineares não nulos. Então:

$$
\cap_{j=1}^{n} N\left(\phi_{j}\right) \subset N(\phi) \Leftrightarrow \exists \lambda_{1}, \ldots, \lambda_{n} \in \mathbb{C} \text { tais que } \phi=\sum_{j=1}^{n} \lambda_{j} \phi_{j} .
$$

Demonstração. $(\Leftarrow)$ Suponha que existam $\lambda_{1}, \ldots, \lambda_{n} \in \mathbb{C}$ tais que $\phi=\sum_{j=1}^{n} \lambda_{j} \phi_{j}$. Seja $x \in \cap_{j=1}^{n} N\left(\phi_{j}\right)$, ou seja,

$$
\phi_{j}(x)=0, j=1, \ldots, n,
$$

e assim

$$
\phi(x)=\sum_{j=1}^{n} \lambda_{j} \phi_{j}(x)=0
$$

então $\cap_{j=1}^{n} N\left(\phi_{j}\right) \subset N(\phi)$.

$(\Rightarrow)$ Faremos por indução. No caso $n=1$ temos $\phi, \phi_{1}: X \rightarrow \mathbb{C}$ operadores lineares e suponha $N\left(\phi_{1}\right) \subset N(\phi)$. Fixe $x_{0} \in X$ tal que $\phi_{1}\left(x_{0}\right)=1$ e seja $x \in X$ qualquer, assim

$$
x=\left(x-\phi_{1}(x) x_{0}\right)+\phi_{1}(x) x_{0} .
$$


Dessa forma,

$$
\phi_{1}\left(x-\phi_{1}(x) x_{0}\right)=\phi_{1}(x)-\phi_{1}(x)=0,
$$

ou seja, $x-\phi_{1}(x) x_{0} \in N\left(\phi_{1}\right) \subset N(\phi)$ e como $x_{0} \notin N\left(\phi_{1}\right)$ segue que $X=N\left(\phi_{1}\right) \oplus\left[x_{0}\right]$. Logo

$$
\phi(x)=\phi\left(x-\phi_{1}(x) x_{0}\right)=\phi\left(x_{0}\right) \phi_{1}(x)
$$

como $x \in X$ é arbitrário concluímos que $\phi=\phi\left(x_{0}\right) \phi_{1}$.

Suponha, por indução, que o resultado seja válido para $k=1, \ldots, n-1$ e provemos para $k=n$. Sejam $\phi, \phi_{1}, \ldots, \phi_{n} \in X^{*}$ tais que $\cap_{j=1}^{n} N\left(\phi_{j}\right) \subset N(\phi)$. Observe que $N\left(\phi_{n}\right) \subset$ $X$ é subespaço vetorial e considere

$$
\left.\phi_{j}^{*} \doteq \phi_{j}\right|_{N\left(\phi_{n}\right)}, j=1, \ldots, n-1 \text { e } \phi^{*} \doteq \phi_{N\left(\phi_{n}\right)}
$$

$\operatorname{Assim} \phi^{*}, \phi_{1}^{*}, \ldots, \phi_{n-1}^{*} \in\left(N\left(\phi_{n}\right)\right)^{*}$ e se $x \in \cap_{j=1}^{n-1} N\left(\phi_{j}^{*}\right)$ então

$$
\begin{aligned}
x & \in N\left(\phi_{n}\right) \text { e } \phi_{j}^{*}(x)=\phi_{j}(x)=0 \Rightarrow x \in \cap_{j=1}^{n} N\left(\phi_{j}\right) \subset N(\phi) \Rightarrow \\
\Rightarrow \quad x & \in N\left(\phi_{n}\right) \text { e } \phi^{*}(x)=\phi(x)=0 \Rightarrow x \in N\left(\phi^{*}\right),
\end{aligned}
$$

ou seja, $\cap_{j=1}^{n-1} N\left(\phi_{j}^{*}\right) \subset N\left(\phi^{*}\right)$.

Pela hipótese de indução, existem $\alpha_{1}, \ldots, \alpha_{n-1} \in \mathbb{C}$ tais que $\phi^{*}=\sum_{j=1}^{n-1} \alpha_{j} \phi_{j}^{*}$. Seja $x_{0} \in X$ tal que $\phi_{n}\left(x_{0}\right)=1$. Logo, para $x \in X$, tem-se que $x=\left(x-\phi_{n}(x) x_{0}\right)+\phi_{n}(x) x_{0}$ $\operatorname{com} x-\phi_{n}(x) x_{0} \in N\left(\phi_{n}\right)$. Dessa forma

$$
\begin{aligned}
\phi(x) & =\phi\left(x-\phi_{n}(x) x_{0}\right)-\phi_{n}(x) \phi\left(x_{0}\right)= \\
& =\phi^{*}\left(x-\phi_{n}(x) x_{0}\right)+\phi\left(x_{0}\right) \phi_{n}(x)= \\
& =\sum_{j=1}^{n-1} \alpha_{j} \phi_{j}^{*}\left(x-\phi_{n}(x) x_{0}\right) \phi\left(x_{0}\right) \phi_{n}(x)= \\
& =\sum_{j=1}^{n-1} \phi_{j}\left(x-\phi_{n}(x) x_{0}\right)-\phi\left(x_{0}\right) \phi_{n}(x)= \\
& =\left(\sum_{j=1}^{n-1} \alpha_{j} \phi_{j}+\left(\phi\left(x_{0}\right)-\sum_{j=1}^{n-1} \alpha_{j} \phi_{j}\left(x_{0}\right)\right) \phi_{n}\right)(x),
\end{aligned}
$$

ou seja,

$$
\phi=\sum_{j=1}^{n} \lambda_{j} \phi_{j},
$$

sendo que $\lambda_{j}=\alpha_{j}, j=1, \ldots, n-1$ e $\lambda_{n}=\phi\left(x_{0}\right)-\sum_{j=1}^{n-1} \alpha_{j} \phi_{j}\left(x_{0}\right)$.

Seja $\mathcal{V} \subset \mathbb{C} T \Omega$ uma estrurura localmente integrável, considere $U \subset \Omega$ não vazio e $L, M \in \mathfrak{X}(U)$ seções de $\mathcal{V}$ sobre $U$. Fixe $p_{0} \in U$, mostremos que $[L, M]_{p_{0}} \in \mathcal{V}_{p_{0}}$. Com 
efeito, existem $Z_{1}, \ldots, Z_{m} \in C^{\infty}(U)$ e $L_{1}, \ldots, L_{n} \in \mathfrak{X}(U)$ tais que

$$
\begin{aligned}
& \mathcal{V}_{p}^{\perp}=\left[d Z_{1 p}, \ldots, d Z_{m p}\right], \forall p \in U, \\
& \mathcal{V}_{p}=\left[L_{1 p}, \ldots, L_{n p}\right], \forall p \in U .
\end{aligned}
$$

Observe que $[L, M]_{p_{0}}, L_{j p_{0}}: C^{\infty}\left(p_{0}\right) \rightarrow \mathbb{C}$ são aplicações $\mathbb{C}$-lineares, para $j=$ $1, \ldots, n$, e denote $N_{j} \doteq N\left(L_{j p_{0}}\right)$. Seja $\underline{f} \in \cap_{j=1}^{n} N_{j} \subset C^{\infty}\left(p_{0}\right)$, assimi $L_{j p_{0}}(\underline{f})=0, j=$ $1, \ldots, n$. Em outras palavras, $d f_{p_{0}}\left(L_{j p_{0}}\right)=0$, ou seja, $d f_{p_{0}} \in \mathcal{V}_{p_{0}}^{\perp}$ e portanto

$$
d f_{p_{0}}=\sum_{j=1}^{m} \alpha_{j} d Z_{j p_{0}}, \alpha_{1}, \ldots, \alpha_{m} \in \mathbb{C}
$$

Além disso, $[L, M]_{p_{0}}=(L \circ M)_{p_{0}}-(M \circ L)_{p_{0}} \mathrm{e}$

$$
\begin{aligned}
(L \circ M)_{p_{0}}(\underline{f}) & =(L \circ M)(f)\left(p_{0}\right)=d f_{p_{0}}(L \circ M)_{p_{0}}=\sum_{j=1}^{m} \alpha_{j} d Z_{j p_{0}}(L \circ M)_{p_{0}}= \\
& =\sum_{j=1}^{m} \alpha_{j}(L \circ M)\left(Z_{j}\right)\left(p_{0}\right)=\sum_{j=1}^{m} \alpha_{j} L\left(M Z_{j}\right)\left(p_{0}\right)=0 .
\end{aligned}
$$

Analogamente, $(M \circ L)_{p_{0}}(\underline{f})=\sum_{j=1}^{m} \alpha_{j} M\left(L Z_{j}\right)\left(p_{0}\right)=0 \Rightarrow[L, M]_{p_{0}}(\underline{f})=0$, ou seja, $\underline{f} \in N\left([L, M]_{p_{0}}\right)$ e $\cap_{j=1}^{n} N_{j} \subset N\left([L, M]_{p_{0}}\right)$.

Por fim, pelo Lema 6.3.2 concluímos que existem $\lambda_{1}, \ldots, \lambda_{n} \in \mathbb{C}$ tais que

$$
[L, M]_{p_{0}}=\sum_{j=1}^{n} \lambda_{j} L_{j p_{0}}
$$

então $[L, M]_{p_{0}} \in \mathcal{V}_{p_{0}}$, como $p_{0} \in U$ é arbitrário segue que $[L, M]_{p} \in \mathcal{V}_{p}, \forall p \in U$ e $\mathcal{V}$ é involutiva.

Note que o operador de Mizohata do Exemplo 6.2.7 é uma estrutura localmente integrável, pois $\mathcal{V}_{p}^{\perp}=\left[d x_{p}+i p_{2} d t_{p}\right]$ então basta tomar

$$
Z=x+\frac{i}{2} t^{2}
$$

e assim

$$
d Z=\frac{\partial Z}{\partial x} d x+\frac{\partial Z}{\partial t} d t=d x+i t d t
$$

Teorema 6.3.3. Toda estrutura essencialmente real $\mathcal{V}$ é localmente integrável.

Demonstração. Seja $\mathcal{V}$ uma estrutura essencialmente real, dado $p_{0} \in \Omega$ existem $U \subset \Omega$ aberto que contém $p_{0}$ e $L_{1}, \ldots, L_{n} \in \mathfrak{X}(U)$ reais tais que $\mathcal{V}_{p}=\left[L_{1 p}, \ldots, L_{n p}\right], \forall p \in U$. Logo $L_{1}, \ldots, L_{n}$ são campos reais que geram a estrutura involutiva $\cup_{p \in U} \mathcal{V}_{p}$, assim pelo Teorema de Frobenius para Variedades Suaves, existem $V \subset U$, aberto que contém $p_{0}$, e $y \doteq\left(y_{1}, \ldots, y_{N}\right)$ sistema de coordenadas locais tais que

$$
\mathcal{V}_{p}=\left[\left(\frac{\partial}{\partial y_{1}}\right)_{p}, \ldots,\left(\frac{\partial}{\partial y_{n}}\right)_{p}\right], \forall p \in V .
$$


Tome $Z_{j} \doteq y_{j+n} \in C^{\infty}(V), j=1, \ldots, m$. Assim

$$
d Z_{j}\left(\frac{\partial}{\partial y_{k}}\right)=d y_{j+m}\left(\frac{\partial}{\partial y_{k}}\right)=\delta_{j+m k}=0, j=1, \ldots, n, k=1, \ldots, m,
$$

ou seja, $d Z_{j p} \in \mathcal{V}_{p}^{\perp}, j=1, \ldots, m, p \in V$, como $\left\{d Z_{j p}\right\}_{j=1}^{m}$ é linearmente independente e $\operatorname{dim}_{p}^{\perp}=m, p \in V$, segue que

$$
\mathcal{V}_{p}^{\perp}=\left[d Z_{1 p}, \ldots, d Z_{m p}\right], p \in V
$$

\subsection{Geradores Locais}

Agora, sendo $\mathcal{V}$ uma estrutura localmente integrável, obteremos um sistema de coordenadas locais de $\Omega$ e geradores locais para $\mathcal{V}^{\perp}$ de forma a simplificar as expressões de seções $L \in \mathfrak{X}(U)$ de $\mathcal{V}$ e de $Z \in C^{\infty}(U)$, em que $d Z_{p} \in \mathcal{V}_{p}^{\perp}, p \in U$, e $U \subset \Omega$ é um aberto não vazio.

Sejam $\mathcal{V}$ uma estrutura localmente integrável e $p_{0} \in \Omega$. Assim existem $U \subset \Omega$, que contém $p_{0}$, e $G_{1}, \ldots, G_{m} \in C^{\infty}(U)$ tais que:

$$
\mathcal{V}_{p}^{\perp}=\left[d G_{1 p}, \ldots, d G_{m p}\right], \forall p \in U
$$

Considere $\left\{\zeta_{1}, \ldots, \zeta_{\nu}\right\}$ base de $V_{1}$ e $\left\{\xi_{\nu+1}, \ldots, \xi_{m}\right\}$ base de $T_{p_{0}}^{0}$, sendo que $V_{1} \subset \mathcal{V}_{p_{0}}$ é subespaço vetorial tal que $\mathcal{V}_{p_{0}}=\left(T_{p_{0}}^{0} \oplus i T_{p_{0}}^{0}\right) \oplus V_{1}, d=\operatorname{dim}_{\mathbb{R}} T_{p_{0}}^{0}$ e $\nu=m-d$. Logo pela Observação 6.2.11, tem-se que

$$
\left\{\zeta_{1}, \ldots, \zeta_{\nu}, \xi_{\nu+1}, \ldots, \xi_{m}\right\} \text { é base de } \mathcal{V}_{p_{0}}^{\perp}
$$

então existe $\left(c_{j k}\right)_{1 \leq j, k \leq m} \in G L(m ; \mathbb{C})$ tal que

$$
\begin{aligned}
\sum_{k=1}^{m} c_{j k} d G_{k p_{0}} & =\zeta_{j}, j=1, \ldots, \nu \\
\sum_{k=1}^{m} c_{j k} d G_{k p_{0}} & =\xi_{j}, j=\nu+1, \ldots, m .
\end{aligned}
$$

Observação 6.4.1. Note que se $f, g \in C^{\infty}(U), \alpha \in \mathbb{C}$, então

$$
d(f+\alpha g)(L)=L(f+\alpha g)=L(f)+\alpha L(g)=(d f+\alpha d g)(L), L \in \mathfrak{X}(U),
$$

ou seja, $d(f+\alpha g)=d f+\alpha d g$. Além disso, se $f(p)=c, p \in U$, onde $c \in \mathbb{C}$, então

$$
d f(L)=L f=0, L \in \mathfrak{X}(U),
$$

$e \operatorname{assim} d f=0$. 
Tome

$$
\begin{aligned}
Z_{j}(p) & \doteq \sum_{k=1}^{m} c_{j k}\left[G_{k}(p)-G_{k}\left(p_{0}\right)\right], p \in U, j=1, \ldots, \nu \\
W_{l}(p) & \doteq \sum_{k=1}^{m} c_{l+\nu k}\left[G_{k}(p)-G_{k}\left(p_{0}\right)\right], p \in U, l=1, \ldots, d
\end{aligned}
$$

Dessa forma,

$$
\begin{aligned}
d Z_{j} & =d\left(\sum_{k=1}^{m} c_{j k}\left(G_{k}-G_{k}\left(p_{0}\right)\right)\right)=\sum_{k=1}^{m} c_{j k} d G_{k}, j=1, \ldots, \nu \\
d W_{l} & =d\left(\sum_{k=1}^{m} c_{l+\nu k}\left(G_{k}-G_{k}\left(p_{0}\right)\right)\right)=\sum_{k=1}^{m} c_{l+\nu k} d G_{k}, l=1, \ldots, d .
\end{aligned}
$$

Se $p \in U$, então

$$
\begin{aligned}
d Z_{j p} & =\sum_{k=1}^{m} c_{j k} d G_{k p}, j=1, \ldots, \nu \\
d W_{l p} & =\sum_{k=1}^{m} c_{l+\nu k} d G_{k p}, l=1, \ldots, d
\end{aligned}
$$

Note que a matriz

$$
\left(\begin{array}{cccccc}
c_{11} & \cdots & c_{\nu 1} & c_{\nu+11} & \cdots & c_{m 1} \\
c_{12} & \cdots & c_{\nu 2} & c_{\nu+12} & \cdots & c_{m 2} \\
\vdots & \ddots & \vdots & \vdots & \ddots & \vdots \\
c_{1 m} & \cdots & c_{\nu m} & c_{\nu+1 m} & \cdots & c_{m m}
\end{array}\right)
$$

representa os coeficientes dos vetores $d Z_{1 p}, \ldots, d Z_{\nu p}, d W_{1 p}, \ldots, d W_{d p}$ na base $\left\{d G_{k p}\right\}_{k=1}^{m}$. Como $\left(c_{j k}\right)_{1 \leq j, k \leq m} \in G L(m ; \mathbb{C})$, então tal matriz é invertível, portanto

$$
\left\{d Z_{1 p}, \ldots, d Z_{\nu p}, d W_{1 p}, \ldots, d W_{d p}\right\} \text { é base de } \mathcal{V}_{p}^{\perp}, p \in U .
$$

Além disso,

$$
\begin{aligned}
d Z_{j p_{0}} & =\sum_{k=1}^{m} c_{j k} d G_{k p_{0}}=\zeta_{j}, j=1, \ldots, \nu \\
d W_{l p_{0}} & =\sum_{k=1}^{m} c_{j+l k} d G_{k p_{0}}=\xi_{j+l}, j=1, \ldots, d
\end{aligned}
$$

Tome $x_{j} \doteq \Re Z_{j}, y_{j} \doteq \Im Z_{j}, j=1, \ldots, \nu, s_{l} \doteq \Re W_{l}, l=1, \ldots, d$. Assim $x_{j}, y_{j}, s_{l} \in$ $C^{\infty}(U ; \mathbb{R}), j=1, \ldots, \nu, l=1, \ldots, d \mathrm{e}$

$$
\begin{aligned}
d x_{j p} & =\Re d Z_{j p}, d y_{j p}=\Im d Z_{j p}, j=1, \ldots, \nu, \\
d s_{l p} & =\Re d W_{l p}, l=1, \ldots, d,
\end{aligned}
$$

para $p \in U$. Logo

$$
\begin{aligned}
d x_{j p_{0}} & =\Re d Z_{j p_{0}}=\Re \zeta_{j}, d y_{j p_{0}}=\Im d Z_{j p_{0}}=\Im \zeta_{j}, j=1, \ldots, \nu \\
d s_{l p_{0}} & =\Re d W_{l p_{0}}=\xi_{l+\nu}, l=1, \ldots, d,
\end{aligned}
$$


e

$$
\begin{gathered}
\left\{d x_{1 p_{0}}, \ldots, d x_{\nu p_{0}}, d y_{1 p_{0}}, \ldots, d y_{\nu p_{0}}, d s_{1 p_{0}}, \ldots, d s_{d p_{0}}\right\} \quad \text { é LI sobre } \mathbb{R}, \\
T_{p_{0}}^{0}=\left[d s_{1 p_{0}}, \ldots, d s_{d p_{0}}\right] .
\end{gathered}
$$

Teorema 6.4.2. Seja $\mathcal{V}$ uma estrutura localmente integrável definida sobre $\Omega$. Considere $p_{0} \in \Omega$ e $d \doteq \operatorname{dim}_{\mathbb{R}} T_{p_{0}}^{0}$, então existem um sistema de coordenadas locais

$$
\left\{x_{1}, \ldots, x_{\nu}, y_{1}, \ldots, y_{\nu}, s_{1}, \ldots, s_{d}, t_{1}, \ldots, t_{n^{\prime}}\right\}
$$

$\operatorname{com} x_{j}\left(p_{0}\right)=y_{j}\left(p_{0}\right)=s_{l}\left(p_{0}\right)=t_{k}\left(p_{0}\right)=0, j=1, \ldots, \nu, l=1, \ldots, d, k=1, \ldots, n^{\prime}, e$ funções reais e suaves $\phi_{1}, \ldots, \phi_{d}$, definidas numa vizinhança de $p_{0}$ tais que

$$
\phi_{k}\left(p_{0}\right)=0, d_{x} \phi_{k p_{0}}=0, k=1, \ldots, d .
$$

Além disso, as diferenciais das seguintes funções

$$
\begin{aligned}
Z_{j}(x, y) & \doteq x_{j}+i y_{j}, j=1, \ldots, \nu \\
W_{k}(x, y, s, t) & \doteq s_{k}+i \phi_{k}(x, y, s, t), k=1, \ldots, d
\end{aligned}
$$

geram $\mathcal{V}^{\perp}$ numa vizinhança de $p_{0}$. Em particular, tem-se que $\nu+d=m, \nu+n^{\prime}=n e$

$$
T_{p_{0}}^{0}=\left[d s_{1 p_{0}}, \ldots, d s_{d p_{0}}\right] .
$$

Demonstração. Considere $n^{\prime}=n-\nu,(U, \psi)$ um sistema de coordenadas locais com $p_{0} \in U$ e $\psi\left(p_{0}\right)=0 \in \mathbb{R}^{N}$. Consequentemente, tem-se que:

$$
\begin{aligned}
d x_{j} & =\sum_{k=1}^{N} \frac{\partial x_{j}}{\partial \psi_{k}} d \psi_{k}, j=1, \ldots, \nu \\
d y_{j} & =\sum_{k=1}^{N} \frac{\partial y_{j}}{\partial \psi_{k}} d \psi_{k}, j=1, \ldots, \nu \\
d s_{l} & =\sum_{k=1}^{N} \frac{\partial s_{l}}{\partial \psi_{k}} d \psi_{k}, l=1, \ldots, d .
\end{aligned}
$$

Denote

$$
\begin{aligned}
b_{j k}(p) & \doteq \frac{\partial x_{j}}{\partial \psi_{k}}(p), j=1, \ldots, \nu \\
b_{j+\nu k}(p) & \doteq \frac{\partial y_{j}}{\partial \psi_{k}}(p), j=1, \ldots, \nu, \\
b_{j+2 \nu k}(p) & \doteq \frac{\partial s_{j}}{\partial \psi_{k}}(p), j=1, \ldots, d,
\end{aligned}
$$

para $p \in U, k=1, \ldots, N$. Note que tais funções são suaves e, de 6.1 , temos que a matriz

$$
\left(\begin{array}{cccc}
b_{11}\left(p_{0}\right) & b_{21}\left(p_{0}\right) & \cdots & b_{m+\nu 1}\left(p_{0}\right) \\
b_{12}\left(p_{0}\right) & b_{22}\left(p_{0}\right) & \cdots & b_{m+\nu 2}\left(p_{0}\right) \\
\vdots & \vdots & \ddots & \vdots \\
b_{1 N}\left(p_{0}\right) & b_{2 N}\left(p_{0}\right) & \cdots & b_{m+\nu N}\left(p_{0}\right)
\end{array}\right)
$$


$p \in U$, tem posto $m+\nu$. Portanto, renomeando os índices se necessário, obtemos que a seguinte matriz $B(p)$ dada por

$$
\left(\begin{array}{cccc}
b_{11}(p) & b_{21}(p) & \cdots & b_{m+\nu 1}(p) \\
b_{12}(p) & b_{22}(p) & \cdots & b_{m+\nu 2}(p) \\
\vdots & \vdots & \ddots & \vdots \\
b_{1 m+\nu}(p) & b_{2 m+\nu}(p) & \cdots & b_{m+\nu m+\nu}(p)
\end{array}\right)
$$

tem determinante não nulo em $p_{0}$.

$$
\begin{aligned}
& \text { Tome } t_{j} \doteq \psi_{m+\nu+j}, j=1, \ldots, n^{\prime} \text { e } f: U \rightarrow \mathbb{R}^{N} \text { por: } \\
& f(p) \doteq\left(x_{1}(p), \ldots, x_{\nu}(p), y_{1}(p), \ldots, y_{\nu}(p), s_{1}(p), \ldots, s_{d}(p), t_{1}(p), \ldots, t_{n^{\prime}}(p)\right), p \in U .
\end{aligned}
$$

Note que $D f\left(p_{0}\right)$ é dada por

$$
\left(\begin{array}{ccccccc}
b_{11}\left(p_{0}\right) & b_{12}\left(p_{0}\right) & \cdots & b_{1 m+\nu}\left(p_{0}\right) & b_{1 m+\nu+1}\left(p_{0}\right) & \cdots & b_{1 N}\left(p_{0}\right) \\
b_{21}\left(p_{0}\right) & b_{22}\left(p_{0}\right) & \cdots & b_{2 m+\nu}\left(p_{0}\right) & b_{2 m+\nu+1}\left(p_{0}\right) & \ldots & b_{2 N}\left(p_{0}\right) \\
\vdots & \vdots & \ddots & \vdots & \vdots & \ddots & \vdots \\
b_{m+\nu 1}\left(p_{0}\right) & b_{m+\nu 2}\left(p_{0}\right) & \cdots & b_{m+\nu m+\nu}\left(p_{0}\right) & b_{m+\nu m+\nu+1}\left(p_{0}\right) & \cdots & b_{m+\nu N}\left(p_{0}\right) \\
0 & 0 & \cdots & 0 & 1 & \cdots & 0 \\
\vdots & \vdots & \ddots & \vdots & \vdots & \ddots & \vdots \\
0 & 0 & \cdots & 0 & 0 & \cdots & 1
\end{array}\right)
$$

e então $\operatorname{det} D f\left(p_{0}\right)=\operatorname{det} B^{t}\left(p_{0}\right) \operatorname{det}\left(I d_{N-(m+\nu)}\right)=\operatorname{det} B\left(p_{0}\right) \neq 0$, onde $I d_{N-(m-\nu)}$ é a matriz identidade de $\mathbb{R}^{N-(m+\nu)}$.

Logo, pelo Teorema da Função Inversa, existe $V \subset U$ aberto que contém $p_{0}$ tal que $f: V \rightarrow f(V)$ é difeomorfismo e $f(V) \subset \mathbb{R}^{N}$ é aberto.

Para concluir a demonstração, basta tomar $\phi_{k} \doteq \Im W_{k} \in C^{\infty}(V), k=1, \ldots, d$, assim

$$
\begin{array}{r}
\phi_{k}\left(p_{0}\right)=\Im \phi_{k}\left(p_{0}\right)=0, \\
d \phi_{k p_{0}}=\Im \xi_{\nu+k}=0,
\end{array}
$$

$k=1, \ldots, d$.

Nas condições do teorema acima, tem-se que

$$
d W_{k}=\sum_{j=1}^{N} \frac{\partial W_{k}}{\partial f_{j}}, k=1, \ldots, d,
$$

e como $d W_{k p_{0}}=d s_{k p_{0}}=d f_{k+2 \nu p_{0}}$, obtemos que

$$
\frac{\partial W_{k}}{\partial s_{l}}\left(p_{0}\right)=\delta_{k l}, k, l=1, \ldots, d .
$$


Queremos encontrar $\mu_{k k^{\prime}} \in C^{\infty}(U), k, k^{\prime}=1, \ldots, d$, tais que os campos

$$
M_{k} \doteq \sum_{k^{\prime}=1}^{d} \mu_{k k^{\prime}} \frac{\partial}{\partial k^{\prime}}, k=1, \ldots, d,
$$

satisfazem $M_{k}\left(W_{k^{\prime}}\right)=\delta_{k k^{\prime}}$ numa vizinhaça de $p_{0}$, para $k, k^{\prime}=1, \ldots, d$.

Para tanto considere a seguinte função a valores matriciais em que $A(p)$ é dada por

$$
\left(\begin{array}{cccc}
\frac{\partial W_{1}}{\partial s_{1}}(p) & \frac{\partial W_{1}}{\partial s_{2}}(p) & \ldots & \frac{\partial W_{1}}{\partial s_{d}}(p) \\
\frac{\partial W_{2}}{\partial s_{1}}(p) & \frac{\partial W_{2}}{\partial s_{2}}(p) & \ldots & \frac{\partial W_{2}}{\partial s_{d}}(p) \\
\vdots & \vdots & \ddots & \vdots \\
\frac{\partial W_{d}}{\partial s_{1}}(p) & \frac{\partial W_{d}}{\partial s_{2}}(p) & \ldots & \frac{\partial W_{d}}{\partial s_{d}}(p)
\end{array}\right)
$$

$p \in U$, por 6.2 temos que $A_{p_{0}}$ é a matriz identidade e portanto é inversível em $p_{0}$. Como as entradas da matriz $A$ são funções suaves, segue que $A$ uma função suave. Unindo estes fatos obtemos que $A$ é inversível numa vizinhança, $V \subset U$, de $p_{0}$.

Assim para cada $p \in V$, tome $\mu_{k l}(p)$ como sendo o elemento da $l$-ésima linha e $k$-ésima coluna da matriz $A^{-1}(p)$. Logo

$$
\sum_{l=1}^{d} \mu_{k l}(p) \frac{\partial W_{k^{\prime}}}{\partial s_{l}}(p)
$$

será o elemento da $k$-ésima linha e $k^{\prime}$-ésima coluna da matriz $A^{-1}(p) A(p)=I_{d}$, ou seja, será igual a $\delta_{k k^{\prime}}$.

Considere os seguintes campos vetoriais

$$
\begin{aligned}
L_{j} & \doteq \frac{\partial}{\partial \bar{Z}_{j}}-i \sum_{k=1}^{d} \frac{\partial \phi_{k}}{\partial \bar{Z}_{j}} M_{k}, j=1, \ldots, \nu \\
\tilde{L}_{l} & \doteq \frac{\partial}{\partial t_{l}}-i \sum_{k=1}^{d} \frac{\partial \phi_{k}}{\partial t_{l}} M_{k}, l=1, \ldots, n^{\prime} .
\end{aligned}
$$

Proposição 6.4.3. O conjunto $\left\{L_{1 p}, \ldots, L_{\nu p}, \tilde{L}_{1 p}, \ldots, \tilde{L}_{n^{\prime} p}\right\}$ é linearmente independente para todo $p \in V$ e além disso

$$
L_{j} Z_{j^{\prime}}=\tilde{L}_{l} Z_{j^{\prime}}=L_{j} W_{k}=\tilde{L}_{l} W_{k}=0,
$$

para $j, j^{\prime}=1, \ldots, \nu, k=1, \ldots, d, l=1, \ldots, n^{\prime}$.

Demonstração. Com efeito, primeiramente observe que

$$
M_{k} Z_{j}=\sum_{l=1}^{d} \mu_{k l} \frac{\partial Z_{j}}{\partial s_{l}}=0=\frac{\partial Z_{k}}{\partial \overline{Z_{j}}}=\frac{\partial Z_{j}}{\partial t_{l}}
$$

e então $L_{j} Z_{j^{\prime}}=\tilde{L}_{l} Z_{j}=0, j, j^{\prime}=1, \ldots, \nu, l=1, \ldots, n^{\prime}$. Além disso,

$$
\begin{aligned}
L_{j} W_{k} & =\frac{\partial W_{k}}{\partial \overline{Z_{j}}}-i \frac{\partial \phi_{k}}{\partial \bar{Z}_{j}}=\frac{\partial s_{k}}{\partial \overline{Z_{j}}}=\frac{1}{2}\left(\frac{\partial s_{k}}{\partial x_{j}}+i \frac{\partial s_{k}}{\partial y_{j}}\right)=0 \\
\tilde{L}_{l} W_{k} & =\frac{\partial W_{k}}{\partial t_{l}}-i \frac{\partial \phi_{k}}{\partial t_{l}}=\frac{\partial s_{k}}{\partial t_{l}}=0 .
\end{aligned}
$$


Resta mostrar que os campos são linearmente independentes em cada ponto $p \in V$. Sejam $\alpha_{1}, \ldots, \alpha_{\nu}, \beta_{1}, \ldots, \beta_{n^{\prime}} \in \mathbb{C}$ e $p \in V$ tais que

$$
\sum_{j=1}^{\nu} \alpha_{j} L_{j p}+\sum_{l=1}^{n^{\prime}} \beta_{l} \tilde{L}_{l p}=0
$$

assim

$$
\sum_{j=1}^{\nu} \frac{\alpha_{j}}{2} \frac{\partial}{\partial x_{j p}}+i \sum_{j=1}^{\nu} \frac{\alpha_{j}}{2} \frac{\partial}{\partial y_{j p}}-i C \sum_{k^{\prime}=1}^{d} \mu_{k k^{\prime}}(p) \frac{\partial}{\partial s_{k^{\prime} p}}+\sum_{l=1}^{n^{\prime}} \beta_{l} \frac{\partial}{\partial t_{l p}}=0,
$$

sendo que

$$
C=\left(\sum_{j, k=1}^{\nu} \alpha_{j} \frac{\partial \phi_{k}}{\partial \bar{Z}_{j}}(p) \sum_{l, k=1}^{\nu} \beta_{l} \frac{\partial \phi_{k}}{\partial t_{l}}(p)\right)
$$

Como $\left(x_{1}, \ldots, x_{\nu}, y_{1}, \ldots, y_{\nu}, s_{1}, \ldots, s_{d}, t_{1}, \ldots, t_{n^{\prime}}\right)$ é um sistema de coordenadas locais segue que $\alpha_{j}=\beta_{l}=0, j=1, \ldots, \nu, l=1, \ldots, n^{\prime}$.

Segue da proposição acima que

$$
d Z_{j^{\prime} p}\left(L_{j p}\right)=d W_{k p}\left(L_{j p}\right)=d Z_{j^{\prime} p}\left(\tilde{L}_{l p}\right)=d W_{k p}\left(\tilde{L}_{l p}\right)=0
$$

para $j, j^{\prime}=1, \ldots, \nu, k=1, \ldots, d, l=1, \ldots, n^{\prime}, p \in V$.

Dessa forma,

$$
\left\{L_{1 p}, \ldots, L_{\nu p}, \tilde{L}_{1 p}, \ldots, \tilde{L}_{n^{\prime} p}\right\} \subset \mathcal{V}_{p}
$$

e como $\mathcal{V}_{p}$ tem dimensão igual $n=\nu+n^{\prime}$ obtemos que

$$
\mathcal{V}_{p}=\left[L_{1 p}, \ldots, L_{\nu p}, \tilde{L}_{1 p}, \ldots, \tilde{L}_{n^{\prime} p}\right], p \in V
$$

Proposição 6.4.4. Seja $p \in V$, então

$$
\left\{d Z_{1 p}, \ldots, d Z_{\nu p}, d \bar{Z}_{1 p}, \ldots, d \bar{Z}_{\nu p}, d W_{1 p}, \ldots, d W_{d p}, d t_{1 p}, \ldots, d t_{n^{\prime} p}\right\}
$$

é uma base para $\mathbb{C} T_{p}^{*} \Omega$ e

$$
\left\{L_{1}^{b}, \ldots, L_{\nu}^{b}, L_{1}, \ldots, L_{\nu}, \tilde{L}_{1}, \ldots, \tilde{L}_{n^{\prime}}, M_{1}, \ldots, M_{d}\right\}
$$

é a sua base dual, em que

$$
L_{j}^{b} \doteq \frac{\partial}{\partial Z_{j}}-i \sum_{k=1}^{d} \frac{\partial \phi_{k}}{\partial Z_{j}} M_{k}, j=1, \ldots, \nu
$$

Além disso, os campos $L_{1}, \ldots, L_{\nu}, L_{1}^{b}, \ldots, L_{\nu}^{b}, \tilde{L}_{1}, \ldots, \tilde{L}_{n^{\prime}}, M_{1}, \ldots, M_{d}$ são comutativos, ou seja, seu comutador $[\cdot, \cdot]$ é nulo. 
Demonstração. De fato, sejam $p \in V$ e $\alpha_{1}, \ldots, \alpha_{\nu}, \beta_{1}, \ldots, \beta_{\nu}, \gamma_{1}, \ldots, \gamma_{d}, \delta_{1}, \ldots, \delta_{n^{\prime}} \in \mathbb{C}$ tais que

$$
\sum_{j=1}^{\nu} \alpha_{j} d Z_{j p}+\sum_{j=1}^{\nu} \beta_{j} d \bar{Z}_{j p}+\sum_{j=1}^{d} \gamma_{j} d W_{j p}+\sum_{j=1}^{n^{\prime}} \delta_{j} d t_{j p}=0
$$

Assim

$$
\sum_{j=1}^{\nu} \beta_{j} d \bar{Z}_{j p}+\sum_{j=1}^{n^{\prime}} \delta_{j} d t_{j p}=-\sum_{j=1}^{\nu} \alpha_{j} d Z_{j p}-\sum_{j=1}^{d} \gamma_{j} d W_{j p} \in \mathcal{V}_{p}^{\perp}
$$

para cada $l=1, \ldots, n^{\prime}$, calculando em $\tilde{L}_{l p}$ obtemos

$$
\delta_{l}=\sum_{j=1}^{n^{\prime}} \delta_{j} d t_{j p}\left(\tilde{L}_{l p}\right)=0
$$

pois $\tilde{L}_{l p} \in \mathcal{V}_{p}$ e $d \bar{Z}_{j p}\left(\tilde{L}_{l p}\right)=0, j=1, \ldots, \nu$.

Por outro lado, calculando a mesma expressão em $L_{l p}$ obtemos

$$
\beta_{l}=\sum_{j=1}^{\nu} \beta_{j} d \bar{Z}_{j p}\left(L_{l p}\right)=0, l=1, \ldots, \nu
$$

Logo

$$
\sum_{j=1}^{\nu} \alpha_{j} d Z_{j p}+\sum_{j=1}^{d} \gamma_{j} d W_{j p}=0
$$

como os vetores $d Z_{1 p}, \ldots, d Z_{\nu p}, d W_{1 p}, \ldots, d W_{d p}$ formam uma base para $\mathcal{V}_{p}^{\perp}$ concluímos que $\alpha_{1}, \ldots, \alpha_{\nu}, \gamma_{1}, \ldots, \gamma_{d}$ são todos nulos.

Mostremos agora que 6.4 é base dual de 6.3. Para tanto, seja $p \in V$. Assim

$$
\begin{gathered}
d Z_{k p}\left(L_{l p}^{b}\right)=\delta_{j k}, d Z_{k p}\left(L_{j p}\right)=0, l, j, k=1, \ldots, \nu \\
d Z_{k p}\left(\tilde{L}_{l p}\right)=0, d Z_{k p}\left(M_{j p}\right)=0, k=1, \ldots, \nu, j=1, \ldots, d, l=1, \ldots, n^{\prime} \\
d \bar{Z}_{k p}\left(L_{l p}^{b}\right)=0, d \bar{Z}_{k p}\left(L_{j p}\right)=\delta_{j k}, l, j, k=1, \ldots, \nu \\
d \bar{Z}_{k p}\left(\tilde{L}_{l p}\right)=0, d \bar{Z}_{k p}\left(M_{j p}\right)=0, k=1, \ldots, \nu, j=1, \ldots, d, l=1, \ldots, n^{\prime} \\
d W_{k p}\left(L_{l p}^{b}\right)=0, d W_{k p}\left(L_{j p}\right)=0, l, j=1, \ldots, \nu, k=1, \ldots, d \\
d W_{k p}\left(\tilde{L}_{l p}\right)=0, d W_{k p}\left(M_{j p}\right)=\delta_{j k}, j, k=1, \ldots, d, l=1, \ldots, n^{\prime} \\
d t_{k p}\left(L_{l p}^{b}\right)=0, d t_{k p}\left(L_{j p}\right)=0, k=1, \ldots, n^{\prime}, j=1, \ldots, \nu \\
d t_{k p}\left(\tilde{L}_{l p}\right)=\delta_{k l}, d t_{k p}\left(M_{j p}\right)=0, j=1, \ldots, d, l, k=1, \ldots, n^{\prime},
\end{gathered}
$$

que é suficiente para concluir que 6.4 é a base dual de 6.3 . 
Por fim, observe que tais campos são comutativos entre si. Com efeito, como qualquer campo $L \in \mathfrak{X}(U)$ pode ser escrito da forma

$$
L_{p}=\sum_{j=1}^{\nu} \alpha_{j} L_{j p}^{b}+\sum_{j=1}^{\nu} \beta_{j} L_{j p}+\sum_{l=1}^{n^{\prime}} \xi_{l} \tilde{L}_{j p}+\sum_{k=1}^{\nu} \zeta_{k} L_{k p}^{b},
$$

com

$$
\begin{aligned}
\alpha_{j} & =d Z_{j}(L)(p), \beta_{j}=d \bar{Z}_{j}(L)(p), j=1, \ldots, \nu \\
\xi_{l} & =d t_{l}(L)(p), l=1, \ldots, n^{\prime}, \zeta_{k}=d W_{k}(L)(p), k=1, \ldots, d,
\end{aligned}
$$

basta mostrar que as diferenciais dos comutadores dos campos se anulam em cada $p \in V$.

Note que

$$
\begin{aligned}
& d Z_{k}\left(\left[L_{j}, L_{j^{\prime}}\right]\right)=d t_{l}\left(\left[L_{j}, L_{j^{\prime}}\right]\right)=d W_{k^{\prime}}\left(\left[L_{j}, L_{j^{\prime}}\right]=0,\right. \\
& d \bar{Z}_{k}\left(\left[L_{j}, L_{j^{\prime}}\right]\right)=L_{j}\left(\delta_{k j^{\prime}}\right)-L_{j^{\prime}}\left(\delta_{k j}\right)=0, \\
& d \bar{Z}_{k}\left(\left[L^{b} j, L_{j^{\prime}}^{b}\right]\right)=d t_{l}\left(\left[L_{j}^{b}, L_{j^{\prime}}^{b}\right]\right)=d W_{k^{\prime}}\left(\left[L_{j}^{b}, L_{j^{\prime}}^{b}\right]=0,\right. \\
& d Z_{k}\left(\left[L_{j}^{b}, L_{j^{\prime}}^{b}\right]\right)=L_{j}^{b}\left(\delta_{k j^{\prime}}\right)-L_{j^{\prime}}^{b}\left(\delta_{k j}\right)=0, \\
& d Z_{k}\left(\left[\tilde{L} j, \tilde{L} j^{\prime}\right]\right)=d \bar{Z}_{l}\left(\left[\tilde{L}_{j}, \tilde{L}_{j^{\prime}}\right]\right)=d W_{k^{\prime}}\left(\left[\tilde{L}_{j}, \tilde{L}_{j^{\prime}}\right]=0,\right. \\
& d t_{k}\left(\left[\tilde{L}_{j}, \tilde{L}_{j^{\prime}}\right]\right)=\tilde{L}_{j}\left(\delta_{k j^{\prime}}\right)-\tilde{L}_{j^{\prime}}\left(\delta_{k j}\right)=0, \\
& d Z_{k}\left(\left[M_{j}, M_{j^{\prime}}\right]\right)=d t_{l}\left(\left[M_{j}, M_{j^{\prime}}\right]\right)=d \bar{Z}_{k^{\prime}}\left(\left[M_{j}, M_{j^{\prime}}\right]=0,\right. \\
& d W_{k}\left(\left[M_{j}, M_{j^{\prime}}\right]\right)=M_{j}\left(\delta_{k j^{\prime}}\right)-M_{j^{\prime}}\left(\delta_{k j}\right)=0, \\
& d t_{l}\left(\left[L_{j}, L_{j^{\prime}}^{b}\right]\right)=d W_{k^{\prime}}\left(\left[L_{j}, L_{j^{\prime}}^{b}\right]=0,\right. \\
& d \bar{Z}_{k}\left(\left[L_{j}, L_{j^{\prime}}^{b}\right]\right)=L_{j}(0)-L_{j^{\prime}}^{b}\left(\delta_{k j}\right)=0, \\
& d Z_{k}\left(\left[L_{j}, L_{j^{\prime}}^{b}\right]\right)=L_{j}\left(\delta_{k j^{\prime}}\right)-L_{j^{\prime}}^{b}(0)=0, \\
& d Z_{k}\left(\left[L_{j}, \tilde{L}_{j^{\prime}}\right]\right)=d W_{k^{\prime}}\left(\left[L_{j}, L_{j^{\prime}}^{b}\right]=0,\right. \\
& d \bar{Z}_{k}\left(\left[L_{j}, \tilde{L}_{j^{\prime}}\right]\right)=L_{j}(0)-\tilde{L}_{j^{\prime}}\left(\delta_{k j}\right)=0 \text {, } \\
& d t_{l}\left(\left[L_{j}, \tilde{L}_{j^{\prime}}\right]\right)=L_{j}\left(\delta_{l j^{\prime}}\right)-\tilde{L}_{j^{\prime}}(0)=0, \\
& d \bar{Z}_{k}\left(\left[L_{j}^{b}, \tilde{L}_{j^{\prime}}\right]\right)=d W_{k^{\prime}}\left(\left[L_{j}^{b}, L_{j^{\prime}}^{b}\right]=0,\right. \\
& d Z_{k}\left(\left[L_{j}^{b}, \tilde{L}_{j^{\prime}}\right]\right)=L_{j}^{b}(0)-\tilde{L}_{j^{\prime}}\left(\delta_{k j}\right)=0 \text {, } \\
& d t_{l}\left(\left[L_{j}^{b}, \tilde{L}_{j^{\prime}}\right]\right)=L_{j}^{b}\left(\delta_{l j^{\prime}}\right)-\tilde{L}_{j^{\prime}}(0)=0,
\end{aligned}
$$




$$
\begin{aligned}
d Z_{k}\left(\left[L_{j}, M_{j^{\prime}}\right]\right) & =d t_{l}\left(\left[L_{j}, M j^{\prime}\right]=0,\right. \\
d \bar{Z}_{k}\left(\left[L_{j}, M_{j^{\prime}}\right]\right) & =L_{j}(0)-M_{j^{\prime}}\left(\delta_{k j}\right)=0, \\
d W_{k^{\prime}}\left(\left[L_{j}, M_{j^{\prime}}\right]\right) & =L_{j}\left(\delta_{k^{\prime} j^{\prime}}\right)-M_{j^{\prime}}(0)=0, \\
d \bar{Z}_{k}\left(\left[L_{j}^{b}, M_{j^{\prime}}\right]\right) & =d t_{l}\left(\left[L_{j}^{b}, M j^{\prime}\right]=0,\right. \\
d Z_{k}\left(\left[L_{j}^{b}, M_{j^{\prime}}\right]\right) & =L_{j}^{b}(0)-M_{j^{\prime}}\left(\delta_{k j}\right)=0, \\
d W_{k^{\prime}}\left(\left[L_{j}^{b}, M_{j^{\prime}}\right]\right) & =L_{j}^{b}\left(\delta_{k^{\prime} j^{\prime}}\right)-M_{j^{\prime}}(0)=0, \\
d \bar{Z}_{k}\left(\left[\tilde{L}_{j}, M_{j^{\prime}}\right]\right) & =d Z_{k}\left(\left[\tilde{L}_{j}, M j^{\prime}\right]=0,\right. \\
d t_{l}\left(\left[\tilde{L}_{j}, M_{j^{\prime}}\right]\right) & =\tilde{L}_{j}(0)-M_{j^{\prime}}\left(\delta_{l j}\right)=0, \\
d W_{k^{\prime}}\left(\left[\tilde{L}_{j}, M_{j^{\prime}}\right]\right) & =\tilde{L}_{j}\left(\delta_{k^{\prime} j^{\prime}}\right)-M_{j^{\prime}}(0)=0,
\end{aligned}
$$

mostrando que todos os colchetes são nulos e concluindo a demonstração.

Como consequência do Teorema 6.4.2, obtemos o seguinte resultado.

Corolário 6.4.5. Seja $\mathcal{V}$ uma estrutura localmente integrável definida sobre $\Omega$. Considere $p_{0} \in \Omega$ e $d \doteq \operatorname{dim}_{\mathbb{R}} T_{p_{0}}^{0}$, então existem um sistema de coordenadas locais

$$
\left\{x_{1}, \ldots, x_{m}, t_{1}, \ldots, t_{n}\right\}
$$

$\operatorname{com} x_{j}\left(p_{0}\right)=t_{k}\left(p_{0}\right)=0, j=1, \ldots, m, k=1, \ldots, n$, e funções reais, suaves $\phi_{1}, \ldots, \phi_{m}$, definidas numa vizinhança de $p_{0}$ tais que

$$
\phi_{k}\left(p_{0}\right)=0, d_{x} \phi_{k p_{0}}=0, k=1, \ldots, m \text {. }
$$

Além disso, as diferenciais das seguintes funções

$$
Z_{j}(x, t) \doteq x_{j}+i \phi_{j}(x, t), j=1, \ldots, m
$$

geram $\mathcal{V}^{\perp}$ numa vizinhança de $p_{0}$.

Demonstração. Sejam $\mathcal{V}$ uma estrutura localmente integrável definida sobre $\Omega$ e $p_{0} \in \Omega$. Pelo Teorema 6.4.2, existem $U \subset \Omega$ aberto que contém $p_{0}$, funções $Z_{1}, \ldots, Z_{\nu}, W_{1}, \ldots, W_{d}$ $\in C^{\infty}(U)$ tais que

$$
\mathcal{V}_{p}^{\perp}=\left[d Z_{1}, \ldots, d Z_{\nu}, d W_{1}, \ldots, d W_{d}\right], p \in U
$$

Além disso, existem $\phi_{k}\left(p_{0}\right)=d \phi_{k p_{0}}=0, k=1, \ldots, d$, a função

$$
f \doteq\left(x_{1}, \ldots, x_{\nu}, s_{1}, \ldots, s_{d}, t_{1}, \ldots, t_{n^{\prime}}, y_{1}, \ldots, y_{\nu}\right)
$$

forma um sistema de coordenadas locais com $f\left(p_{0}\right)=0$, onde $n^{\prime}=n-\nu$,

$$
x_{j}=\Re Z_{j}, y_{j}=\Im Z_{j}, j=1, \ldots, \nu, s_{k}=\Re W_{k}
$$


e ainda vale

$$
T_{p_{0}}^{0}=\left[d s_{1}, \ldots, d s_{d}\right]
$$

Tome

$$
\begin{array}{r}
x_{j+\nu} \doteq s_{j}, j=1, \ldots, d, \\
\phi_{k} \doteq y_{k}, k=1, \ldots, \nu, \\
\phi_{k+\nu} \doteq \Im W_{k}, k=1, \ldots, d, \\
t_{j+n^{\prime}} \doteq y_{j}, j=1, \ldots, \nu, \\
Z_{k+\nu} \doteq W_{k}, k=1, \ldots, d .
\end{array}
$$

Assim

$$
\begin{aligned}
f & =\left(x_{1}, \ldots, x_{m}, t_{1}, \ldots, t_{n}\right) \\
Z_{j} & =x_{j}+i \phi_{j}, j=1, \ldots, m, \\
\phi_{k}\left(p_{0}\right) & =0, k=1, \ldots, m
\end{aligned}
$$

e

$$
\begin{aligned}
& \mathcal{V}_{p}^{\perp}=\left[d Z_{1 p}, \ldots, d Z_{m p}\right], p \in U \\
& \frac{\partial \phi_{k}}{\partial x_{j}}\left(p_{0}\right)=0, j, k=1, \ldots, m
\end{aligned}
$$

Nas condições do teorema acima, denote

$$
Z(x, t) \doteq\left(Z_{1}(x, t), \ldots, Z_{m}(x, t)\right)
$$

Dessa maneira, tem-se que

$$
\frac{\partial Z_{j}}{\partial x_{k}}\left(p_{0}\right)=\delta_{j k}, j, k=1, \ldots, m
$$

pois $d_{x} \phi_{k p_{0}}=0, k=1, \ldots, m$, ou seja, $Z_{x}\left(p_{0}\right)=I d_{m}$.

Pelo mesmo argumento utilizado acima, existem, numa vizinhança $U$ de $p_{0}$, funções $\mu_{k l} \in C^{\infty}(U)$ tais que $M_{k}\left(Z_{j}\right)=\delta_{j k}, j, k=1, \ldots, m$, onde

$$
M_{k} \doteq \sum_{l=1}^{m} \mu_{k l} \frac{\partial}{\partial x_{l}} .
$$

Defina os campos

$$
L_{j} \doteq \frac{\partial}{\partial t_{j}}-i \sum_{l=1}^{m} \frac{\partial \phi_{l}}{\partial t_{j}} M_{l}, j=1, \ldots, n .
$$

Então temos o seguinte resultado: 
Proposição 6.4.6. Considere os conjuntos $S \doteq\left\{L_{1 p}, \ldots, L_{n p}, M_{1 p}, \ldots, M_{m p}\right\}$ e $S^{*} \doteq$ $\left\{d Z_{1 p}, \ldots, d Z_{m p}, d t_{1 p}, \ldots, d t_{n p}\right\}$. Então $S$ é linearmente independente em $\mathbb{C} T_{p} \Omega$ e $S^{*} e ́$ linearmente independente em $\mathbb{C} T_{p}^{*} \Omega$, para todo $p \in U$, vale

$$
\begin{gathered}
L_{j} Z_{k}=M_{k} t_{j}=0, j=1, \ldots, n, k=1, \ldots, m, \\
\left\{d Z_{1 p}, \ldots, d Z_{m p}, d t_{1 p}, \ldots, d t_{n p}\right\}
\end{gathered}
$$

é uma base para $\mathbb{C} T_{p}^{*} \Omega$ e

$$
\left\{L_{1 p}, \ldots, L_{n p}, M_{1 p}, \ldots, M_{m p}\right\}
$$

é a sua base dual. Além disso, os campos $L_{1}, \ldots, L_{n}, M_{1}, \ldots, M_{m}$ são comutativos, ou seja, seu comutador $[\cdot, \cdot]$ é nulo.

Demonstração. Inicialmente, observe que $M_{k} t_{j}=0, L_{j} Z_{k}=0, M_{k} Z_{l}=\delta_{k l}$ e $L_{j} t_{k}=\delta_{j k}$, pois

e

$$
\frac{\partial t_{j}}{\partial x_{l}}=0
$$

$$
\frac{\partial Z_{k}}{\partial t_{j}}-i \sum_{l=1}^{m} \frac{\partial \phi_{l}}{\partial t_{j}} M_{l} Z_{k}=i \frac{\partial \phi_{k}}{\partial t_{j}}-i \frac{\partial \phi_{k}}{\partial t_{j}}=0 .
$$

Sejam $p \in U, \alpha_{1}, \ldots, \alpha_{n}, \beta_{1}, \ldots, \beta_{m} \in \mathbb{C}$ tais que

$$
\sum_{j=1}^{n} \alpha_{j} d t_{j p}+\sum_{k=1}^{m} \beta_{k} d Z_{k p}=0
$$

Calculando em $L_{j p}$ obtemos que $\alpha_{j}=0, j=1, \ldots, n$ e calculando em $M_{k p}$ obtemos que $\beta_{k}=0, k=1, \ldots, m$.

Assim $S^{*}$ é linearmente independente e como tal conjunto tem $N$ elementos, concluímos que tal conjunto é base de $\mathbb{C} T_{p}^{*} \Omega$.

Agora como $d Z_{k p}\left(L_{j p}\right)=L_{j}\left(Z_{k}\right)(p)=0, d t_{j p}\left(M_{k p}\right)=M_{k}\left(t_{j}\right)(p)=0, d Z_{k p}\left(M_{j p}\right)=$ $M_{j}\left(Z_{k}\right)(p)=\delta_{j k}, d t_{j p}\left(L_{k p}\right)=L_{k}\left(t_{j}\right)(p)=\delta_{j k}$ e do fato de $S^{*}$ ser base de $\mathbb{C} T_{p}^{*} \Omega$, segue que $S$ é sua base dual.

Por fim, mostremos que os campos são comutativos, para concluirmos este fato, como em 6.5, é suficiente mostrar que as diferenciais dos comutadores se anulam.

Note que

$$
\begin{aligned}
d Z_{k}\left(\left[L_{j}, L_{j^{\prime}}\right]\right) & =0, d Z_{k}\left(L_{j}, M_{j^{\prime}}\right)=L_{j}\left(\delta_{k j^{\prime}}\right)-M_{j^{\prime}}(0)=0, \\
d Z_{k}\left(\left[M_{j}, M_{j^{\prime}}\right]\right) & =M_{j}\left(\delta_{k j^{\prime}}\right)-M_{j^{\prime}}\left(\delta_{k j}\right)=0 \\
d t_{k}\left(\left[M_{j}, M_{j^{\prime}}\right]\right) & =0, d t_{k}\left(L_{j}, M_{j^{\prime}}\right)=L_{j}(0)-M_{j^{\prime}}\left(\delta_{k j}\right)=0, \\
d t_{k}\left(\left[L_{j}, L_{j^{\prime}}\right]\right) & =L_{j}\left(\delta_{k j^{\prime}}\right)-L_{j^{\prime}}\left(\delta_{k j}\right)=0,
\end{aligned}
$$

como queríamos demonstrar. 
Observação 6.4.7. Observe que se estivermos nas condições da proposição acima, segue que

$$
\mathcal{V}_{p}=\left[L_{1 p}, \ldots, L_{n p}\right], p \in U
$$

pois $d Z_{k p}\left(L_{j p}\right)=0$ e $\left\{L_{1 p}, \ldots, L_{n p}\right\}$ é linearmente independente.

A seguir apresentaremos alguns lemas que são de suma importância na demonstração da versão clássica do Teorema de Baouendi-Treves.

Primeiramente, se $\zeta=\left(\zeta_{1}, \ldots, \zeta_{m}\right) \in \mathbb{C}^{m}$ denotamos

$$
[\zeta]^{2} \doteq \sum_{j=1}^{m} \zeta_{j}^{2}
$$

suponha que $\Omega$ é um aberto de $\mathbb{R}^{N}$ que contém a origem, $\mathcal{L}$ é uma estrutura localmente integrável sobre $\Omega$ e fixe $p_{0}=0$. Observe que estamos nas condições de 6.4.5 e 6.4.6.

Para cada $\tau \in(0,+\infty)$, considere o seguinte operador:

$$
G_{\tau} u(x, t) \doteq\left(\frac{\tau}{\pi}\right)^{m / 2} \int_{\mathbb{R}^{m}} e^{-\tau\left[Z(x, t)-Z\left(x^{\prime}, t\right)\right]^{2}} u\left(x^{\prime}, t\right) h\left(x^{\prime}\right) \operatorname{det} Z_{x}\left(x^{\prime}, t\right) d x^{\prime}, u \in C^{1}(W),
$$

onde $h \in C_{c}^{\infty}\left(\mathbb{R}^{m}\right)$ com $h(x)=0,|x| \geq R$ e $h=1$ numa vizinhança do conjunto

$$
\left\{x \in \mathbb{R}^{m} ;|x| \leq R / 2\right\}
$$

tal $R$ será determinado mais adiante, $W$ é aberto de $\Omega$ que contém a origem em que a função $Z=\left(Z_{1}, \ldots, Z_{m}\right)$ está definida e $Z_{x}(x, t)$ é a matriz jacobiana na variável $x$ desta função calculada em $(x, t) \in W$.

Se $L \in \mathfrak{X}(W)$ denote

$$
\begin{aligned}
& {\left[G_{\tau}, L\right] \doteq G_{\tau}(L)-L\left(G_{\tau}\right),} \\
& {\left[L, G_{\tau}\right] \doteq L\left(G_{\tau}\right)-G_{\tau}(L) .}
\end{aligned}
$$

Lema 6.4.8. Para $u \in C^{1}(W)$ e $k=1, \ldots, m$ vale

$$
\left[M_{k}, G_{\tau}\right] u(x, t)=\left(\frac{\tau}{\pi}\right)^{m / 2} \int_{\mathbb{R}^{m}} e^{-\tau\left[Z(x, t)-Z\left(x^{\prime}, t\right)\right]^{2}} u\left(x^{\prime}, t\right) M_{k} h\left(x^{\prime}\right) \operatorname{det} Z_{x}\left(x^{\prime}, t\right) d x^{\prime} .
$$

Demonstração. Observe que se

$$
F: \mathbb{C}^{m} \rightarrow \mathbb{C}
$$

for uma função inteira, $g \in C\left(W ; \mathbb{C}^{m}\right), L \in \mathfrak{X}(W)$ e $\xi$ denota o sistema de coordendas locais de $W$, então

$$
L=\sum_{j=1}^{N} a_{j} \frac{\partial}{\partial \xi_{j}}, a_{j} \in C^{\infty}(W),
$$


e como

$$
\begin{aligned}
\frac{\partial(F \circ g)}{\partial \xi_{j}}(p) & =\frac{\partial\left(F \circ g \circ \xi^{1}\right)}{\partial e_{j}} \circ \xi(p)=F^{\prime}(g(p)) \frac{\partial\left(g \circ \xi^{-1}\right)}{\partial e_{j}} \circ \xi(p)= \\
& =\left(F^{\prime} \circ g \frac{\partial g}{\partial \xi_{j}}\right)(p), p \in W, j=1, \ldots, N
\end{aligned}
$$

$\operatorname{assim} L(F \circ g)=\left(F^{\prime} \circ g\right) L g$.

Denotaremos por $M_{k}\left(x^{\prime}, t, D_{x^{\prime}}\right)$ o operador $M_{k}$ atuando nas variáveis $\left(x^{\prime}, t\right)$, ou seja,

$$
M_{k}\left(x^{\prime}, t, D_{x^{\prime}}\right) \doteq \sum_{l=1}^{m} \mu_{k l}\left(x^{\prime}, t\right) \frac{\partial}{\partial x_{l}^{\prime}}, k=1, \ldots, m .
$$

Dessa maneira, para $k, j=1, \ldots, m$ tem-se que

$$
M_{k}\left(x, t, D_{x}\right)\left(Z_{j}(x, t)-Z_{j}\left(x^{\prime}, t\right)\right)=\delta_{j k}-\sum_{l=1}^{m} \mu_{k l}(x, t) \frac{\partial\left(x_{j}^{\prime}+i \phi_{j}\left(x^{\prime}, t\right)\right)}{\partial x_{l}}=\delta_{j k},
$$

analogamente

$$
M_{k}\left(x^{\prime}, t, D_{x^{\prime}}\right)\left(Z_{j}(x, t)-Z_{j}\left(x^{\prime}, t\right)\right)=-\delta_{j k},
$$

$\log 0$

$$
M_{k}\left(x, t, D_{x}\right)\left(Z_{j}(x, t)-Z_{j}\left(x^{\prime}, t\right)\right)=-M_{k}\left(x^{\prime}, t, D_{x^{\prime}}\right)\left(Z_{j}(x, t)-Z_{j}\left(x^{\prime}, t\right)\right)=\delta_{j k} .
$$

Seja $F(\zeta) \doteq e^{-\tau[\zeta]^{2}}$, que é uma função inteira. Então

$$
\begin{gathered}
M_{k}\left(x, t, D_{x}\right)\left(F\left(Z(x, t)-Z\left(x^{\prime}, t\right)\right)\right)= \\
=-2 \tau\left(F\left(Z(x, t)-Z\left(x^{\prime}, t\right)\right)\left(\sum_{j=1}^{m}\left(Z_{j}(x, t)-Z_{j}\left(x^{\prime}, t\right)\right)\right) M_{k}\left(x, t, D_{x}\right)\left(Z_{j}(x, t)-Z_{j}\left(x^{\prime}, t\right)\right),\right.
\end{gathered}
$$

analogamente

$$
\begin{gathered}
M_{k}\left(x^{\prime}, t, D_{x^{\prime}}\right)\left(F\left(Z(x, t)-Z\left(x^{\prime}, t\right)\right)\right)= \\
=-2 \tau\left(F\left(Z(x, t)-Z\left(x^{\prime}, t\right)\right)\left(\sum_{j=1}^{m}\left(Z_{j}(x, t)-Z_{j}\left(x^{\prime}, t\right)\right)\right) M_{k}\left(x^{\prime}, t, D_{x^{\prime}}\right)\left(Z_{j}(x, t)-Z_{j}\left(x^{\prime}, t\right)\right),\right.
\end{gathered}
$$

e de 6.6 conclui-se que

$$
M_{k}\left(x, t, D_{x}\right)\left(F\left(Z(x, t)-Z\left(x^{\prime}, t\right)\right)\right)=-M_{k}\left(x^{\prime}, t, D_{x^{\prime}}\right)\left(F\left(Z(x, t)-Z\left(x^{\prime}, t\right)\right)\right) .
$$

O fato de $h$ ter suporte compacto faz com que, para qualquer função $u \in C^{1}(W)$, $G_{\tau} u$ tenha suporte compacto e portanto é uma função uniformemente contínua na variável $x$, assim podemos derivar sob o sinal de integração e obter que

$$
\begin{aligned}
& M_{k} G_{\tau} u(x, t)= \\
& =\left(\frac{\tau}{\pi}\right)^{m / 2} \int_{\mathbb{R}^{m}} \quad M_{k}\left(x, t, D_{x}\right)\left(F\left(Z(x, t)-Z\left(x^{\prime}, t\right)\right)\right) u\left(x^{\prime}, t\right) h\left(x^{\prime}\right) \operatorname{det} Z_{x}\left(x^{\prime}, t\right) d x^{\prime}= \\
& =\left(\frac{\tau}{\pi}\right)^{m / 2} \int_{\mathbb{R}^{m}}-M_{k}\left(x^{\prime}, t, D_{x^{\prime}}\right)\left(F\left(Z(x, t)-Z\left(x^{\prime}, t\right)\right)\right) u\left(x^{\prime}, t\right) h\left(x^{\prime}\right) \operatorname{det} Z_{x}\left(x^{\prime}, t\right) d x^{\prime} .
\end{aligned}
$$


Afirmamos que para cada $(x, t) \in W$ vale

$$
\int_{\mathbb{R}^{m} \times\{t\}} M_{k}(v) w d Z=-\int_{\mathbb{R}^{m} \times\{t\}} M_{k}(w) v d Z,
$$

em que $v, w \in C^{1}(W)$ e uma delas tem suporte compacto.

Agora note que

$$
\begin{aligned}
& M_{k} G_{\tau} u(x, t)= \\
& =\left(\frac{\tau}{\pi}\right)^{m / 2} \int_{\mathbb{R}^{m}}-M_{k}\left(x^{\prime}, t, D_{x^{\prime}}\right) F\left(Z(x, t)-Z\left(x^{\prime}, t\right)\right) u\left(x^{\prime}, t\right) h\left(x^{\prime}\right) \operatorname{det} Z_{x}\left(x^{\prime}, t\right) d x^{\prime}= \\
& =\left(\frac{\tau}{\pi}\right)^{m / 2} \int_{\mathbb{R}^{m}}-i_{t}^{*}\left(M_{k}\left(x^{\prime}, t, D_{x^{\prime}}\right) F\left(Z(x, t)-Z\left(x^{\prime}, t\right)\right)\right)\left(i_{t}^{*} u\right)\left(x^{\prime}\right) i_{t}^{*} h\left(x^{\prime}\right)\left(i_{t}^{*} d Z\right)\left(x^{\prime}\right),
\end{aligned}
$$

sendo que $i_{t}^{*}$ denota o pull-back pela função $i_{t}(x)=(x, t)$.

Portanto, a expressão acima é igual a

$$
\begin{gathered}
\left(\frac{\tau}{\pi}\right)^{m / 2} \int_{\mathbb{R}^{m} \times\{t\}}-M_{k}\left(x^{\prime}, t, D_{x^{\prime}}\left(F\left(Z(x, t)-Z\left(x^{\prime}, t\right)\right)\right)\right) u\left(x^{\prime}, t\right) h\left(x^{\prime}\right) d Z= \\
\left.=\left(\frac{\tau}{\pi}\right)^{m / 2} \int_{\mathbb{R}^{m} \times\{t\}} F\left(Z(x, t)-Z\left(x^{\prime}, t\right)\right)\right) M_{k}\left(u\left(x^{\prime}, t\right) h\left(x^{\prime}\right)\right) d Z= \\
\left.=\left(\frac{\tau}{\pi}\right)^{m / 2} \int_{\mathbb{R}^{m} \times\{t\}} F\left(Z(x, t)-Z\left(x^{\prime}, t\right)\right)\right)\left(M_{k}\left(u\left(x^{\prime}, t\right)\right) h\left(x^{\prime}\right)+u\left(x^{\prime}, t\right) M_{k} h\left(x^{\prime}\right)\right) d Z= \\
=G_{\tau} M_{k} u(x, t)+\left(\frac{\tau}{\pi}\right)^{m / 2} \int_{\mathbb{R}^{m}} e^{-\tau\left[Z(x, t)-Z\left(x^{\prime}, t\right)\right]^{2}} u\left(x^{\prime}, t\right) M_{k} h\left(x^{\prime}\right) \operatorname{det} Z_{x}\left(x^{\prime}, t\right) d x^{\prime},
\end{gathered}
$$

concluindo o lema.

Resta provar que 6.7 é verdadeira.

Sejam $v, w \in C^{1}(W)$ uma delas com suporte compacto e considere, para cada $k=1, \ldots, m$, a seguinte $m$-forma é uma diferencial exata

$$
\begin{aligned}
\omega_{k} & \doteq d\left(u v d Z_{1} \wedge \ldots \wedge \hat{d Z_{k}} \wedge \ldots \wedge d Z_{m}\right)= \\
& =d(u v) \wedge d Z_{1} \wedge \ldots \wedge \hat{d Z_{k}} \wedge \ldots \wedge d Z_{m}
\end{aligned}
$$

ou seja, $\omega_{k}$ é a derivada exterior da $(m-1)$-forma diferencial

$$
\tilde{\omega}_{k} \doteq u v d Z_{1} \wedge \ldots \wedge \hat{d Z_{k}} \wedge \ldots \wedge d Z_{m}
$$

Como $u v \in C^{1}(W)$, tem-se que

$$
d(u v)=\sum_{j=1}^{n} L_{j}(u v) d t_{j}+\sum_{l=1}^{m} M_{l}(u v) d Z_{l},
$$

$\log 0$

$$
\omega_{k}=\left(\sum_{j=1}^{n} L_{j}(u v) d t_{j}+\sum_{l=1}^{m} M_{l}(u v) d Z_{l}\right) \wedge d Z_{1} \wedge \ldots \wedge \hat{d} Z_{k} \wedge \ldots \wedge d Z_{m} .
$$

Além disso, temos $i_{t}^{*} d t_{j}=d\left(i_{t}^{*} t_{j}\right)=0, j=1, \ldots, n$, e assim $i_{t}^{*}\left(d t_{j} \wedge d Z_{1} \wedge \ldots \wedge \hat{d} Z_{k} \wedge \ldots \wedge d Z_{m}\right)=d\left(i_{t}^{*} t_{j}\right) \wedge d\left(i_{t}^{*} Z_{1}\right) \wedge \ldots \wedge \hat{d} Z_{k} \wedge \ldots \wedge d\left(i_{t}^{*} Z\right)_{m}=0$ 
Dessa forma,

$$
\begin{aligned}
i_{t}^{*} \omega_{k} & =i_{t}^{*}\left(\sum_{j=1}^{n} L_{j}(u v) d t_{j} \wedge d Z_{1} \wedge \ldots \wedge \hat{d} Z_{k} \wedge \ldots \wedge d Z_{m}+\right. \\
& \left.+\sum_{l=1}^{m} M_{l}(u v) d Z_{l} \wedge d Z_{1} \wedge \ldots \wedge \hat{d} Z_{k} \wedge \ldots \wedge d Z_{m}\right)= \\
& =i_{t}^{*} M_{l}(u v) i_{t}^{*}\left(d Z_{k} \wedge d Z_{1} \wedge \ldots \wedge \hat{d} Z_{k} \wedge \ldots \wedge d Z_{m}\right)= \\
& =(-1)^{k+1} i_{t}^{*} M_{k}(u v) i_{t}^{*} d Z
\end{aligned}
$$

e então

$$
\int_{\mathbb{R}^{m} \times\{t\}} \omega_{k}=\int_{\mathbb{R}^{m}} i_{t}^{*} \omega_{k}=\int_{\mathbb{R}^{m}} i_{t}^{*} d \tilde{\omega}_{k}=\int_{\mathbb{R}^{m}} d\left(i_{t}^{*} \tilde{\omega}_{k}\right)=0 .
$$

Em outras palavras, obtemos que

$$
\begin{aligned}
\int_{\mathbb{R}^{m}} i_{t}^{*}\left(v M_{k} u+u M_{k} v\right) i_{t}^{*} d Z & =(-1)^{k+1} \int_{\mathbb{R}^{m}}(-1)^{k+1} i_{t}^{*}\left(v M_{k} u+u M_{k} v\right) i_{t}^{*} d Z \\
& =(-1)^{k+1} \int_{\mathbb{R}^{m}} d\left(i_{t}^{*} \tilde{\omega}_{k}\right)=0 .
\end{aligned}
$$

Finalmente, obtemos que

$$
\begin{aligned}
\int_{\mathbb{R}^{m} \times\{t\}} v M_{k} u d Z=\int_{\mathbb{R}^{m}} i_{t}^{*}\left(v M_{k} u\right) i_{t}^{*} d Z & =-\int_{\mathbb{R}^{m}} i_{t}^{*}\left(u M_{k} v\right) i_{t}^{*} d Z= \\
& =-\int_{\mathbb{R}^{m} \times\{t\}} u M_{k} v d Z,
\end{aligned}
$$

concluindo a demonstração.

Antes de enunciarmos o próximo lema, vamos simplificar a notação dos campos $L_{j}$. Tais campos são da forma

$$
L_{j}=\frac{\partial}{\partial t_{j}}-i \sum_{k, l=1}^{m} \frac{\partial \phi_{l}}{\partial t_{j}} \mu_{l k} \frac{\partial}{\partial x_{k}}
$$

ou seja,

$$
L_{j}=\frac{\partial}{\partial t_{j}}+\sum_{k=1}^{m} \lambda_{j k} \frac{\partial}{\partial x_{k}}
$$

em que

$$
\lambda_{j k} \doteq-i \sum_{l=1}^{m} \frac{\partial \phi_{l}}{\partial t_{j}} \mu_{l k}, j=1, \ldots, n, k=1, \ldots, m
$$

Vamos calcular o operador transposto de $L_{j}$, ou seja, o operador denotado por ${ }^{\mathrm{t}} L_{j}$ tal que

$$
\int_{\mathbb{R}^{N}}\left(L_{j} u\right) v d x d t=\int_{\mathbb{R}^{N}} u\left({ }^{\mathrm{t}} L_{j} v\right) d x d t
$$


para quaisquer $u, v$ funções suavves e uma delas com suporte compacto. Observe que

$$
\begin{aligned}
\int_{\mathbb{R}^{N}}\left(L_{j} u\right) v d x d t & =\int_{\mathbb{R}^{N}} \frac{\partial u}{\partial t_{j}} v d x d t+\sum_{k=1}^{m} \int_{\mathbb{R}^{N}} \lambda_{j k} \frac{\partial u}{\partial x_{k}} v d x d t= \\
& =-\int_{\mathbb{R}^{N}} \frac{\partial v}{\partial t_{j}} u d x d t-\sum_{k=1}^{m} \int_{\mathbb{R}^{N}} \frac{\partial\left(\lambda_{j k} v\right)}{\partial x_{k}} u d x d t= \\
& =-\int_{\mathbb{R}^{N}} u\left(\frac{\partial v}{\partial t_{j}}+\sum_{k=1}^{m} \frac{\partial\left(\lambda_{j k} v\right)}{\partial x_{k}}\right) d x d t
\end{aligned}
$$

ou seja,

$$
{ }^{\mathrm{t}} L_{j} v=-\frac{\partial v}{\partial t_{j}}-\sum_{k=1}^{m} \frac{\partial\left(\lambda_{j k} v\right)}{\partial x_{k}} .
$$

Lema 6.4.9. Para cada $j=1, \ldots, n$ vale

$$
\frac{\partial\left(\operatorname{det} Z_{x}\right)}{\partial t_{j}}+\sum_{k=1}^{m} \frac{\partial\left(\lambda_{j k} \operatorname{det} Z_{x}\right)}{\partial x_{k}}=0
$$

Demonstração. Seja $v \in C_{c}^{\infty}\left(\mathbb{R}^{N}\right)$ e considere a $N$-forma a seguir

$$
\begin{aligned}
\omega_{j} & \doteq d\left(v d Z \wedge d t_{1} \wedge \ldots \wedge \hat{d t}_{j} \wedge \ldots \wedge d t_{n}\right)= \\
& =d v \wedge d Z \wedge d t_{1} \wedge \ldots \wedge \hat{d t}_{j} \wedge \ldots \wedge d t_{n}
\end{aligned}
$$

Mas como

$$
d v=\sum_{j=1}^{n} L_{j} v d t_{j}+\sum_{k=1}^{m} M_{k} v d Z_{k}
$$

temos

$$
\begin{aligned}
\omega_{j} & =L_{j} v d t_{j} \wedge d Z \wedge d t_{1} \wedge \ldots \wedge \hat{d t}_{j} \wedge \ldots \wedge d t_{n}= \\
& =(-1)^{m+j-1} L_{j}(v) d Z \wedge d t=(-1)^{m+j-1} L_{j}(v) \operatorname{det} Z_{x} d x \wedge d t
\end{aligned}
$$

Como $\omega_{j}$ é uma $N$-forma exata sua integral em $\mathbb{R}^{N}$ é zero. Além disso,

$$
\begin{aligned}
\int_{\mathbb{R}^{N}} v{ }^{\mathrm{t}} L_{j}\left(\operatorname{det} Z_{x}\right) d x d t & =\int_{\mathbb{R}^{N}}\left(L_{j} v\right) \operatorname{det} Z_{x} d x d t= \\
& =(-1)^{m+j-1} \int_{\mathbb{R}^{N}} \omega_{j}=0
\end{aligned}
$$

isto é,

$$
\int_{\mathbb{R}^{N}} v^{\mathrm{t}} L_{j}\left(\operatorname{det} Z_{x}\right) d x d t=0, \forall v \in C_{c}^{\infty}\left(\mathbb{R}^{N}\right)
$$

e portanto

$$
\frac{\partial\left(\operatorname{det} Z_{x}\right)}{\partial t_{j}}+\sum_{k=1}^{m} \frac{\partial\left(\lambda_{j k} \operatorname{det} Z_{x}\right)}{\partial x_{k}}=-{ }^{\mathrm{t}} L_{j}\left(\operatorname{det} Z_{x}\right)=0
$$

Lema 6.4.10. Para $u \in C^{1}(W)$ e $j=1, \ldots, n$ vale

$$
\left[L_{j}, G_{\tau}\right] u(x, t)=\left(\frac{\tau}{\pi}\right)^{m / 2} \int_{\mathbb{R}^{m}} e^{-\tau\left[Z(x, t)-Z\left(x^{\prime}, t\right)\right]^{2}} u\left(x^{\prime}, t\right) L_{j} h\left(x^{\prime}\right) \operatorname{det} Z_{x}\left(x^{\prime}, t\right) d x^{\prime} .
$$


Observação 6.4.11. Seja $\tilde{g}(\zeta, t)$ uma função suave definida em $\mathbb{C}^{m} \times \mathbb{R}^{n}$, ou seja, holomorfa com respeito a $\zeta \in \mathbb{C}^{m}$ e suave com respeito a $t \in \mathbb{R}^{n}$. Defina

$$
g(x, t) \doteq \tilde{g}(Z(x, t), t),(x, t) \in W .
$$

Então

$$
\frac{\partial g}{\partial t_{j}}\left(x_{0}, t_{0}\right)=\frac{\partial \tilde{g}}{\partial t_{j}}\left(Z\left(x_{0}, t_{0}\right), t_{0}\right)+D_{\zeta} \tilde{g}\left(Z\left(x_{0}, t_{0}\right), t_{0}\right) \frac{\partial Z}{\partial t_{j}}\left(x_{0}, t_{0}\right) .
$$

e

$$
\frac{\partial g}{\partial x_{k}}\left(x_{0}, t_{0}\right)=D_{\zeta} \tilde{g}\left(Z\left(x_{0}, t_{0}\right), t_{0}\right) \frac{\partial Z}{\partial x_{k}}\left(x_{0}, t_{0}\right)
$$

Logo

$$
\begin{aligned}
L_{j} g(x, t) & =\frac{\partial g}{\partial t_{j}}(x, t)+\sum_{k=1}^{m} \lambda_{j k}(x, t) \frac{\partial g}{\partial x_{k}}(x, t)= \\
& =\frac{\partial \tilde{g}}{\partial t_{j}}(Z(x, t), t)+D_{\zeta} \tilde{g}(Z(x, t), t)\left(\frac{\partial Z}{\partial t_{j}}(x, t)+\sum_{k=1}^{m} \lambda_{j k}(x, t) \frac{\partial Z}{\partial x_{k}}(x, t)\right)= \\
& =\frac{\partial \tilde{g}}{\partial t_{j}}(Z(x, t), t)+D_{\zeta} \tilde{g}(Z(x, t), t)\left(L_{j} Z_{1}(x, t), \ldots, L_{j} Z_{m}(x, t)\right)= \\
& =\frac{\partial \tilde{g}}{\partial t_{j}}(Z(x, t), t),(x, t) \in W .
\end{aligned}
$$

Agora faremos a prova do lema anterior.

Demonstração. Para cada $\tau \in(0,+\infty), u \in C^{1}(W)$ o operador

$$
\tilde{G}_{\tau} u(\zeta, t) \doteq \int_{\mathbb{R}^{m}} e^{-\tau\left[\zeta-Z\left(x^{\prime}, t\right)\right]^{2}} u\left(x^{\prime}, t\right) h\left(x^{\prime}\right) \operatorname{det} Z_{x}\left(x^{\prime}, t\right) d x^{\prime}, \zeta \in \mathbb{C}^{m} .
$$

Temos que, se $u \in C^{1}(W)$, então $\tilde{G}_{\tau} u(\zeta, t)$ é uma função holomorfa nas variáveis $\zeta$, suave na variável $t$ e

$$
G_{\tau} u(x, t)=\left(\frac{\tau}{\pi}\right)^{m / 2} \tilde{G}_{\tau} u(Z(x, t), t),(x, t) \in W,
$$

logo, pela observação anterior, obtemos

$$
L_{j}\left(G_{\tau} u\right)(x, t)=\left(\frac{\tau}{\pi}\right)^{m / 2} \frac{\partial \tilde{G}_{\tau} u}{\partial t_{j}}(Z(x, t), t) .
$$

Derivando sob o sinal de integração encontramos

$$
\frac{\partial \tilde{G}_{\tau} u}{\partial t_{j}}(Z(x, t), t)=\left(\frac{\tau}{\pi}\right)^{m / 2} \int_{\mathbb{R}^{m}} \frac{\partial}{\partial t_{j}}\left(e^{-\tau\left[\zeta-Z\left(x^{\prime}, t\right)\right]^{2}} u\left(x^{\prime}, t\right) h\left(x^{\prime}\right) \operatorname{det} Z_{x}\left(x^{\prime}, t\right)\right) d x^{\prime} .
$$

Defina

$$
e_{\tau}\left(\zeta, x^{\prime}, t\right) \doteq e^{-\tau\left[\zeta-Z\left(x^{\prime}, t\right)\right]^{2}}
$$

então

$$
\begin{aligned}
\frac{\partial\left(e_{\tau} u h \operatorname{det} Z_{x}\right)}{\partial t_{j}} & =\frac{\partial\left(e_{\tau} u h\right)}{\partial t_{j}} \operatorname{det} Z_{x}+\frac{\partial\left(\operatorname{det} Z_{x}\right)}{\partial t_{j}} e_{\tau} h u= \\
& =L_{j}\left(e_{\tau} h u\right) \operatorname{det} Z_{x}-\sum_{k=1}^{m} \lambda_{j k} \frac{\partial\left(e_{\tau} u h\right)}{\partial x_{k}} \operatorname{det} Z_{x}+\frac{\partial\left(\operatorname{det} Z_{x}\right)}{\partial t_{j}} e_{\tau} h u
\end{aligned}
$$


Observação 6.4.12. Note que, por 6.8, temos

$$
\begin{aligned}
\frac{\partial \tilde{G}_{\tau} u}{\partial t_{j}}(Z(x, t), t) & =\int_{\mathbb{R}^{m}} L_{j}\left(e_{\tau} h u\right)\left(Z(x, t), x^{\prime}, t\right) \operatorname{det} Z_{x}\left(x^{\prime}, t\right) d x^{\prime}- \\
& -\int_{\mathbb{R}^{m}} \sum_{k=1}^{m} \lambda_{j k}\left(x^{\prime}, t\right) \frac{\partial\left(e_{\tau} u h\right)}{\partial x_{k}}\left(Z(x, t), x^{\prime}, t\right) \operatorname{det} Z_{x}\left(x^{\prime}, t\right) d x^{\prime}+ \\
& +\int_{\mathbb{R}^{m}} \frac{\partial\left(\operatorname{det} Z_{x}\right)}{\partial t_{j}}\left(x^{\prime}, t\right)\left(e_{\tau} h u\right)\left(Z(x, t), x^{\prime}, t\right) d x^{\prime}= \\
& =\int_{\mathbb{R}^{m}} L_{j}\left(e_{\tau} h u\right)\left(Z(x, t), x^{\prime}, t\right) \operatorname{det} Z_{x}\left(x^{\prime}, t\right) d x^{\prime}+ \\
& +\int_{\mathbb{R}^{m}}\left(e_{\tau} h u\right)\left(Z(x, t), x^{\prime}, t\right)\left(\sum_{k=1}^{m} \frac{\partial\left(\lambda_{j k} \operatorname{det} Z_{x}\right)}{\partial x_{k}}\left(x^{\prime}, t\right)\right)+ \\
& +\int_{\mathbb{R}^{m}}\left(e_{\tau} h u\right)\left(Z(x, t), x^{\prime}, t\right) \frac{\partial\left(\operatorname{det} Z_{x}\right)}{\partial t_{j}}\left(x^{\prime}, t\right) d x^{\prime}= \\
& =\int_{\mathbb{R}^{m}} L_{j}\left(e_{\tau} h u\right)\left(Z(x, t), x^{\prime}, t\right) \operatorname{det} Z_{x}\left(x^{\prime}, t\right) d x^{\prime} .
\end{aligned}
$$

Como

$$
L_{j}\left(e_{\tau} u h\right)\left(Z(x, t), x^{\prime}, t\right)=e_{\tau}\left(Z(x, t), x^{\prime}, t\right)\left(L_{j} u\left(x^{\prime}, t\right) h\left(x^{\prime}\right)+u\left(x^{\prime}, t\right) L_{j} h\left(x^{\prime}\right)\right),
$$

pois

$$
L_{j} Z=\left(L_{j} Z_{1}, \ldots, L_{j} Z_{m}\right)=(0, \ldots, 0),
$$

e pela observação acima concluímos

$$
\begin{aligned}
\frac{\partial \tilde{G}_{\tau} u}{\partial t_{j}}(Z(x, t), t) & =\int_{\mathbb{R}^{m}}\left(e_{\tau}\left(L_{j} u\right) h \operatorname{det} Z_{x}\right)\left(Z(x, t), x^{\prime}, t\right) d x^{\prime}+ \\
& +\int_{\mathbb{R}^{m}}\left(e_{\tau} u\left(L_{j} h\right) \operatorname{det} Z_{x}\right)\left(Z(x, t), x^{\prime}, t\right) d x^{\prime}= \\
& =\tilde{G}_{\tau} L_{j} u(Z(x, t), t)+\int_{\mathbb{R}^{m}}\left(e_{\tau} u\left(L_{j} h\right) \operatorname{det} Z_{x}\right)\left(Z(x, t), x^{\prime}, t\right) d x .
\end{aligned}
$$

Portanto, multiplicando a última expressão por $(\tau / \pi)^{m / 2}$ e subtraindo $G_{\tau} L_{j} u$ obtemos

$$
\left[L_{j}, G_{\tau}\right] u(x, t)=\left(\frac{\tau}{\pi}\right)^{m / 2} \int_{\mathbb{R}^{m}} e^{-\tau\left[Z(x, t)-Z\left(x^{\prime}, t\right)\right]^{2}} u\left(x^{\prime}, t\right) L_{j} h\left(x^{\prime}\right) \operatorname{det} Z_{x}\left(x^{\prime}, t\right) d x^{\prime} .
$$

Defina, para cada $u \in C^{1}(W)$, o seguinte operador:

$$
H_{\tau f} u(\zeta, t)=\int_{\mathbb{R}^{m}} e_{\tau}\left(\zeta, x^{\prime}, t\right) u\left(x^{\prime}, t\right) f\left(x^{\prime}\right) \operatorname{det} Z_{x}\left(x^{\prime}, t\right) d x^{\prime},
$$

em que $f \in C_{c}^{\infty}\left(\mathbb{R}^{m}\right)$ tal que $f$ tem suporte contido no suporte de $h$. 
Tem-se que $H_{\tau f} u$ é holomorfa nas variáveis $\zeta$ e suave nas variáveis $t$. Além disso,

$$
\begin{aligned}
G_{\tau} u(x, t) & =\left(\frac{\tau}{\pi}\right)^{m / 2} H_{\tau h} u(Z(x, t), t), \\
{\left[M_{k}, G_{\tau}\right] u(x, t) } & =\left(\frac{\tau}{\pi}\right)^{m / 2} H_{\tau M_{k} h} u(Z(x, t), t), \\
{\left[L_{j}, G_{\tau}\right] u(x, t) } & =\left(\frac{\tau}{\pi}\right)^{m / 2} H_{\tau L_{j} h} u(Z(x, t), t),
\end{aligned}
$$

para $(x, t) \in W, j=1, \ldots, n, k=1, \ldots, m$.

Observe que

$$
L_{j} M_{k} G_{\tau} u=L_{j}\left[M_{k}, G_{\tau}\right] u+L_{j} G_{\tau} M_{k} u
$$

e então

$$
L_{j}\left[M_{k}, G_{\tau}\right] u(x, t)=L_{j}(\tau / \pi)^{m / 2} H_{\tau M_{k} h} u(Z(x, t), t)=(\tau / \pi)^{m / 2} \frac{\partial H_{\tau M_{k} h}}{\partial t_{j}}(Z(x, t), t),
$$

para $j=1, \ldots, n, k=1, \ldots, m$.

Repetindo o mesmo raciocínio para o operador $H_{\tau M_{k} h}$, encontramos

$$
\frac{\partial H_{\tau M_{k} h}}{\partial t_{j}}(Z(x, t), t)=\int_{\mathbb{R}^{m}} \frac{\partial}{\partial t_{j}}\left(e_{\tau}\left(Z(x, t), x^{\prime}, t\right) u\left(x^{\prime}, t\right) M_{k} h\left(x^{\prime}\right) \operatorname{det} Z_{x}\left(x^{\prime}, t\right)\right) d x^{\prime}
$$

e analogamente obtemos

$$
\begin{aligned}
\frac{\partial H_{\tau M_{k} h}}{\partial t_{j}}(Z(x, t), t) & =\int_{\mathbb{R}^{m}} L_{j}\left(e_{\tau} u M_{k} h\right)\left(Z(x, t), x^{\prime}, t\right) \operatorname{det} Z_{x}\left(x^{\prime}, t\right) d x^{\prime}= \\
& =\int_{\mathbb{R}^{m}}\left(e_{\tau} L_{j} u M_{k} \operatorname{det} Z_{x}\right)\left(Z(x, t), x^{\prime}, t\right) d x^{\prime}+ \\
& +\int_{\mathbb{R}^{m}}\left(e_{\tau} u L_{j} M_{k} \operatorname{det} Z_{x}\right)\left(Z(x, t), x^{\prime}, t\right) d x^{\prime}= \\
& =H_{\tau M_{k} h} L_{j}(Z(x, t), t)+H_{\tau L_{j} M_{k} h}(Z(x, t), t) .
\end{aligned}
$$

Por outro lado, note que

$$
M_{k} L_{j} G_{\tau} u=M_{k}\left[L_{j}, G_{\tau}\right] u+M_{k} G_{\tau} L_{j} u
$$

e então

$$
M_{k}\left[L_{j}, G_{\tau}\right] u(x, t)=M_{k}(\tau / \pi)^{m / 2} H_{\tau L_{j} h} u(Z(x, t), t)
$$

para $j=1, \ldots, n, k=1, \ldots, m$. 
Analogamente, para o operador $H_{\tau L_{j} h}$, encontramos

$$
\begin{aligned}
M_{k}\left(x, t, D_{x}\right) \quad H_{\tau L_{j} h} & (Z(x, t), t)= \\
& =\int_{\mathbb{R}^{m}} M_{k}\left(x, t, D_{x}\right)\left(e_{\tau}\right)\left(Z(x, t), x^{\prime}, t\right) u\left(x^{\prime}, t\right) L_{j} h\left(x^{\prime}\right) \operatorname{det} Z_{x}\left(x^{\prime}, t\right) d x^{\prime} \\
& =\int_{\mathbb{R}^{m}}-M_{k}\left(x^{\prime}, t, D_{x^{\prime}}\right)\left(e_{\tau}\right)\left(Z(x, t), x^{\prime}, t\right) u\left(x^{\prime}, t\right) L_{j} h\left(x^{\prime}\right) d Z\left(x^{\prime}, t\right) d x^{\prime}= \\
& =\int_{\mathbb{R}^{m}} e_{\tau}\left(Z(x, t), x^{\prime}, t\right) M_{k}\left(x^{\prime}, t, D_{x^{\prime}}\right)\left(u\left(x^{\prime}, t\right) L_{j} h\left(x^{\prime}\right)\right) d Z\left(x^{\prime}, t\right) d x^{\prime}= \\
& =\int_{\mathbb{R}^{m}} e_{\tau}\left(Z(x, t), x^{\prime}, t\right) M_{k} u\left(x^{\prime}, t\right) L_{j} h\left(x^{\prime}\right) \operatorname{det} Z_{x}\left(x^{\prime}, t\right) d x^{\prime}+ \\
& +\int_{\mathbb{R}^{m}} e_{\tau}\left(Z(x, t), x^{\prime}, t\right) u\left(x^{\prime}, t\right) M_{k} L_{j} h\left(x^{\prime}\right) \operatorname{det} Z_{x}\left(x^{\prime}, t\right) d x^{\prime}= \\
& =H_{\tau L_{j} h} M_{k} u(Z(x, t), t)+H_{\tau M_{k} L_{j}} u(Z(x, t), t) .
\end{aligned}
$$

Observação 6.4.13. Resumidamente, obtivemos que

$$
\begin{aligned}
L_{j} H_{\tau f} u & =H_{\tau L_{j} f} u+H_{\tau f} L_{j} u, \\
M_{k} H_{\tau f} u & =H_{\tau M_{k} f} u+H_{\tau f} M_{k} u .
\end{aligned}
$$

Tais fatos nos levam ao seguinte resultado

Lema 6.4.14. Para quaisquer $s, r \in \mathbb{N}$, vale a igualdade

$$
\begin{aligned}
L_{j_{1}} \ldots L_{j_{s}} M_{k_{1}} \ldots M_{k_{r}} G_{\tau} u & =\left[L_{j_{1}}, G_{\tau}\right] L_{j_{2}} \ldots L_{j_{s}} M_{k_{1}} \ldots M_{k_{r}} u+\ldots \\
& +L_{j_{1}} \ldots L_{j_{s}} M_{k_{1}} \ldots M_{k_{r-1}}\left[M_{k_{r}}, G_{\tau}\right] u+ \\
& +G_{\tau} L_{j_{1}} \ldots L_{j_{s}} M_{k_{1}} \ldots M_{k_{r}} u .
\end{aligned}
$$

Demonstração. A prova será feita por indução finita. Observe que, como os campos são comutativos entre si e como já provamos o caso em que $s, r=1$, basta que provemos a validade da fórmula para uma quantidade $s+r+1$ qualquer de campos.

Denote

$$
\begin{gathered}
L_{J} \doteq L_{j_{1}} \ldots L_{j_{s}}, \\
M_{K} \doteq M_{k_{1}} \ldots M_{k_{r}}
\end{gathered}
$$

Assim

$$
\begin{aligned}
L_{j_{0}} L_{J} M_{K} G_{\tau} u & =L_{j_{0}}\left[L_{j_{1}}, G_{\tau}\right] L_{j_{2}} \ldots L_{j_{s}} M_{k_{1}} \ldots M_{k_{r}} u+\ldots \\
& +L_{j_{0}} L_{j_{1}} \ldots L_{j_{s}} M_{k_{1}} \ldots M_{k_{r-1}}\left[M_{k_{r}}, G_{\tau}\right] u+ \\
& +L j_{0} G_{\tau} L_{j_{1}} \ldots L_{j_{s}} M_{k_{1}} \ldots M_{k_{r}} u= \\
& =\left[L_{j_{0}}, G_{\tau}\right] L_{j_{1}} \ldots L_{j_{s}} M_{k_{1}} \ldots M_{k_{r}} u+ \\
& +L_{j_{0}}\left[L_{j_{1}}, G_{\tau}\right] L_{j_{2}} \ldots L_{j_{s}} M_{k_{1}} \ldots M_{k_{r}} u+\ldots \\
& +L_{j_{0}} L_{j_{1}} \ldots L_{j_{s}} M_{k_{1}} \ldots M_{k_{r-1}}\left[M_{k_{r}}, G_{\tau}\right] u+ \\
& +G_{\tau} L_{j_{0}} L_{j_{1}} \ldots L_{j_{s}} M_{k_{1}} \ldots M_{k_{r}} u .
\end{aligned}
$$


Agora mostraremos que se $u \in C^{\infty}(W)$ então a função $G_{\tau} u$ converge, na topologia $C^{\infty}(U)$, para $u$ quando $\tau \rightarrow \infty$ em que $U \subset \Omega$ que contém a origem.

Para tanto, faremos algumas simplificações. Como $0 \in \Omega$ e para $j, k=1, \ldots, m$, $\left(\partial \phi_{j} / \partial x_{k}\right)(x, t)$ são funções contínuas que se anulam na origem, então existe $R>0$ tal que $\bar{V} \subset W$, onde

$$
V \doteq\left\{(x, t) \in \mathbb{R}^{N} ;|x|<R,|t|<R\right\}
$$

e

$$
\left|\frac{\partial \phi_{j}}{\partial x_{k}}(x, t)\right|<\frac{1}{2},(x, t) \in \bar{V} .
$$

Lema 6.4.15. Seja $B$ uma matriz $m \times m$ com coeficientes reais e norma $\|B\|<1$, então

$$
\operatorname{det}(A) \int_{\mathbb{R}^{m}} e^{-[A x]^{2}} d x=\pi^{m / 2}
$$

em que $A=I d_{m}+i B$.

Observação 6.4.16. De agora em diante, a menos que seja necessário especificar, denotaremos a matriz identidade $I d_{k}$ de $\mathbb{R}^{k}$ apenas por $I$, para qualquer $k \in \mathbb{N}$.

Demonstração. A princípio, observe que

$$
[A x]^{2}=\sum_{j=1}^{m}(A x)_{j}^{2}=\langle A x, A x\rangle,
$$

onde $(A x)_{j}$ denota a $j$-ésima coordenada do vetor $A x$. Afirmamos que

$$
[A x]^{2}=\left\langle{ }^{\mathrm{t}} A A x, x\right\rangle \text {. }
$$

Denote por $a_{j k}$ o elemento da $j$-ésima linha e $k$-ésima coluna de $A$, assim

$$
\begin{aligned}
(A x)_{j} & =\sum_{k=1}^{m} a_{j k} x_{k}, \\
\left({ }^{\mathrm{t}} A A x\right)_{l} & =\sum_{k, j=1}^{m} a_{k l} a_{k j} x_{j},
\end{aligned}
$$

então

$$
\begin{aligned}
\left\langle{ }^{\mathrm{t}} A A x, x\right\rangle & =\sum_{k, j, l=1}^{m} a_{k l} a_{k j} x_{j} x_{l}=\sum_{k=1}^{m}\left(\sum_{l=1}^{m} a_{k l} x_{l}\right)\left(\sum_{j=1}^{m} a_{k j} x_{j}\right) \\
& =\sum_{k=1}^{m}(A x)_{k}^{2}=[A x]^{2} .
\end{aligned}
$$

Defina $C \doteq{ }^{\mathrm{t}} A A$, então

$$
[A x]^{2}=\left\langle{ }^{\mathrm{t}} A A x, x\right\rangle=\langle C x, x\rangle, x \in \mathbb{R}^{m} .
$$


Note que

$$
C={ }^{\mathrm{t}} A A={ }^{\mathrm{t}}(I+i B)(I+i B)=\left(I+i^{\mathrm{t}} B\right)(I+i B)=\left(I+{ }^{\mathrm{t}} B B\right)+i\left({ }^{\mathrm{t}} B+B\right) .
$$

Logo $\Re C=I+{ }^{\mathrm{t}} B B$ é tal que

$$
\left\langle\left(I+{ }^{\mathrm{t}} B B\right) x, x\right\rangle=|x|^{2}-\left\langle{ }^{\mathrm{t}} B B x, x\right\rangle>|x|^{2}-|x|^{2}=0,
$$

pois $\left\|^{\mathrm{t}} B\right\|=\|B\|<1$, ou seja, $C$ é uma matriz complexa simétrica e com parte real positivo definida.

Afirmamos que, para matrizes $M$ simétricas com parte real positivo definida vale

$$
\int_{\mathbb{R}^{m}} e^{-\langle M x, x\rangle} d x=\pi^{m / 2}(\operatorname{det} M)^{-1 / 2},
$$

sendo que o ramo da raiz quadrada é escolhido de forma que $(\operatorname{det} M)^{1 / 2}>0$ quando $M$ é real.

Assumindo a afirmação acima concluimos o resultado, pois

$$
\operatorname{det} C=\operatorname{det}\left({ }^{\mathrm{t}} A A\right)=\operatorname{det}\left({ }^{\mathrm{t}} A\right) \operatorname{det} A=(\operatorname{det} A)^{2} \text {. }
$$

Provemos agora a afirmação acima. Temos que se $a \in(0,+\infty)$ então

$$
\int_{\mathbb{R}^{m}} e^{-a x^{2}} d x=\pi^{m / 2}
$$

Defina

$$
\begin{array}{r}
f(z) \doteq \pi^{1 / 2} z^{-1 / 2}, z \in(0,+\infty)+i \mathbb{R} \\
g(z) \doteq \int_{\mathbb{R}^{m}} e^{-z x^{2}} d x, z \in(0,+\infty)+i \mathbb{R},
\end{array}
$$

tais funções são holomorfas e $f(z)=g(z), z \in(0,+\infty)$ então pelo princípio da identidade (veja [6]) obtemos que $f=g$, isto é,

$$
\int_{\mathbb{R}^{m}} e^{-z x^{2}} d x=\pi^{1 / 2} z^{-1 / 2}, z \in(0,+\infty)+i \mathbb{R} .
$$

Se $M$ for uma matriz hermitiana diagonal com seus elementos da diagonal $a_{j} \in$ $(0,+\infty)+i \mathbb{R}$, então

$$
\begin{aligned}
\int_{\mathbb{R}^{m}} e^{-\langle M x, x\rangle} d x=\Pi_{j=1}^{m} \int_{\mathbb{R}} e^{-a_{j} x_{j}^{2}} d x_{j} & =\Pi_{j=1}^{m} \pi^{m / 2}\left(\operatorname{det} a_{j}\right)^{-1 / 2}= \\
& =\pi^{m / 2}(\operatorname{det} M)^{-1 / 2}
\end{aligned}
$$

Por fim, seja $M$ uma matriz hermitiana simétrica com parte real positivo definida. Tal matriz é diagonalizável por ser simétrica, ou seja, existe matriz $P$ unitária $(|\operatorname{det} P|=$ 
1) tal que $P A P^{-1}$ é diagonal. Se indicarmos por $z_{j}$ o $j$-ésimo elemento da diagonal de $P A P^{-1}$, então $z_{j} \in(0,+\infty)+i \mathbb{R}$ e portanto

$$
\int_{\mathbb{R}^{m}} e^{-\left\langle P A P^{-1} x, x\right\rangle} d x=\pi^{m / 2}\left(\operatorname{det} P A P^{-1}\right)^{-1 / 2}=\pi^{m / 2}(\operatorname{det} A)^{-1 / 2} .
$$

Por outro lado, como $P$ é unitária podemos fazer a mudança de variáveis $y=P^{-1} x$, ou seja,

$$
\int_{\mathbb{R}^{m}} e^{-\left\langle P A P^{-1} x, x\right\rangle} d x=\int_{\mathbb{R}^{m}} e^{-\langle P A y, P y\rangle}|\operatorname{det} P| d y=\int_{\mathbb{R}^{m}} e^{-\langle P A y, P y\rangle} d y
$$

e temos ainda que matrizes unitárias satisfazem

$$
\langle P A y, P y\rangle=\left\langle P^{*} P A y, y\right\rangle=\langle A y, y\rangle, y \in \mathbb{R}^{m},
$$

concluindo a demonstração.

No nosso caso, temos

$$
Z_{x}(x, t)=I+i \phi_{x}(x, t)
$$

$\operatorname{com}\|\phi(x, t)\| \leq 1 / 2<1,(x, t) \in \bar{V}$, logo pelo lema acima

$$
\operatorname{det} Z_{x}(x, t) \int_{\mathbb{R}^{m}} e^{-\left[Z_{x}(x, t) x^{\prime}\right]^{2}} d x^{\prime}=\pi^{m / 2} .
$$

Proposição 6.4.17. Se $u \in C^{\infty}(W)$ então $(\tau / \pi)^{m / 2} H_{\tau f} u$ converge uniformemente para fu em $\bar{V}$, em que $f \in C_{c}^{\infty}\left(\mathbb{R}^{m}\right)$ com suporte contido no suporte de $h$.

Observação 6.4.18. Vale ressaltar que a convergência uniforme da proposição acima ocorre em

$$
\mathbb{R}^{m} \times\{|t| \leq R\}
$$

pois h tem suporte compacto contido em

$$
\{|x|<R\}
$$

daí podemos definir $(h u)(x, t)=0$ se $|x| \geq R,|t| \leq R$. Destacamos também, que segue da proposição acima que, como $h=1$ numa vizinhança de

$$
\{|x| \leq R / 2\}
$$

$G_{\tau} u$ converge uniformemente para $u \mathrm{em}$

$$
\{|x| \leq R / 2\} \times\{|t| \leq R\} .
$$


Demonstração. Defina a função

$$
v(x, t) \doteq f(x) u(x, t) \operatorname{det} Z_{x}(x, t),(x, t) \in \mathbb{R}^{m} \times\{|t| \leq R\} .
$$

Logo, de 6.12 concluimos que

$$
(f u)(x, t)=\pi^{-m / 2} \int_{\mathbb{R}^{m}} e^{-\left[Z_{x}(x, t) x^{\prime}\right]^{2}} v(x, t) d x^{\prime} .
$$

Realizando a mudança de variáveis

$$
x^{\prime} \mapsto x+\tau^{-1 / 2} x^{\prime},
$$

na integral que define $H_{\tau f} u$ obtemos

$$
(\tau / \pi)^{m / 2} H_{\tau f} u(x, t)=\pi^{-m / 2} \int_{\mathbb{R}^{m}} e^{-\tau\left[Z(x, t)-Z\left(x+\tau^{-1 / 2} x^{\prime}, t\right)\right]^{2}} v\left(x+\tau^{-1 / 2} x^{\prime}, t\right) d x^{\prime} .
$$

Dividimos a diferença $H_{\tau f} u(x, t)-f(x) u(x, t)$ em duas integrais, a saber

$$
\begin{aligned}
I_{\tau}(x, t) & \doteq \pi^{-m / 2} \int_{\mathbb{R}^{m}} e^{-\left[Z_{x}(x, t) x^{\prime}\right]^{2}}\left(v\left(x+\tau^{-1 / 2} x^{\prime}, t\right)-v(x, t)\right) d x^{\prime} \\
J_{\tau}(x, t) & \doteq \pi^{-m / 2} \int_{\mathbb{R}^{m}}\left(e^{-\tau\left[Z(x, t)-Z\left(x+\tau^{-1 / 2} x^{\prime}, t\right)\right]^{2}}-e^{-\left[Z_{x}(x, t) x^{\prime}\right]^{2}}\right) v\left(x+\tau^{-1 / 2} x^{\prime}, t\right) d x^{\prime} .
\end{aligned}
$$

É importante destacar que podemos separar essa diferença em duas integrais somando e subtraindo o termo

$$
\int_{\mathbb{R}^{m}} e^{-\left[Z_{x}(x, t) x^{\prime}\right]^{2}} v\left(x+\tau^{-1 / 2} x^{\prime}, t\right) d x^{\prime},
$$

sabemos que tal integral é finita por 6.12 e pois $v$ é contínua, portanto é limitada no compacto

$$
\operatorname{supp}(h) \times\{|t| \leq R\}
$$

Vamos estimar primeiro $\left|I_{\tau}(x, t)\right|$. Observe que

$$
\left|I_{\tau}(x, t)\right| \leq \pi^{-m / 2} \int_{\mathbb{R}^{m}} e^{-\Re\left[Z_{x}(x, t) x^{\prime}\right]^{2}}\left|v\left(x+\tau^{-1 / 2} x^{\prime}, t\right)-v(x, t)\right| d x^{\prime},
$$

assim basta estimarmos tal função exponencial

$$
e^{-\Re\left[Z_{x}(x, t) x^{\prime}\right]^{2}}
$$

e a diferença

$$
v\left(x+\tau^{-1 / 2} x^{\prime}, t\right)-v(x, t),
$$

$\operatorname{para}(x, t) \in \bar{V}, x^{\prime} \in \mathbb{R}^{m}$.

Sabemos que

$$
Z_{x}(x, t) x^{\prime}=x^{\prime}+i \phi_{x}(x, t) x^{\prime}
$$


então

$$
\begin{aligned}
-\left[Z_{x}(x, t) x^{\prime}\right]^{2} & =-\sum_{j=1}^{m}\left(x_{j}^{\prime}+i\left(\phi_{x}(x, t) x^{\prime}\right)_{j}\right)^{2}= \\
& =\sum_{j=1}^{m}\left(-x_{j}^{\prime 2}+\left(\phi_{x}(x, t) x^{\prime}\right)_{j}{ }^{2}-2 i x_{j}^{\prime}\left(\phi_{x}(x, t) x^{\prime}\right)_{j}\right)= \\
& =-\left|x^{\prime}\right|^{2}+\left|\phi_{x}(x, t) x^{\prime}\right|^{2}+i(-2) \sum_{j=1}^{m} x_{j}^{\prime}\left(\phi_{x}(x, t) x^{\prime}\right)_{j}
\end{aligned}
$$

isto é,

$$
-\Re\left[Z_{x}(x, t) x^{\prime}\right]^{2}=-\left|x^{\prime}\right|^{2}+\left|\phi_{x}(x, t) x^{\prime}\right|^{2} .
$$

Logo,

$$
-\Re\left[Z_{x}(x, t) x^{\prime}\right]^{2} \leq-\left|x^{\prime}\right|^{2}+\frac{1}{4}\left|x^{\prime}\right|^{2}=-\frac{3}{4}\left|x^{\prime}\right|^{2}
$$

e assim

$$
\left|e^{-\Re\left[Z_{x}(x, t) x^{\prime}\right]^{2}}\right| \leq e^{-\frac{3}{4}\left|x^{\prime}\right|^{2}},(x, t) \in \bar{V}, x^{\prime} \in \mathbb{R}^{m} .
$$

Observação 6.4.19. Temos que v é uma função suave, então pelo Teorema Fundamental do Cálculo vale

$$
\left.v\left(x+\tau^{-1 / 2} x^{\prime}, t\right)-v(x, t)=\int_{0}^{1} \frac{\partial}{\partial s}(v(\lambda(s), t)) d s=\int_{0}^{1} \nabla_{x} v(\lambda(s), t)\right) \tau^{-1 / 2} x^{\prime} d s,
$$

em que $\lambda(s)=x+s \tau^{-1 / 2} x^{\prime}, x, x^{\prime} \in \mathbb{R}^{m},|t| \leq R$.

Além disso, sabemos que, para cada $|t| \leq R$,

$$
\operatorname{supp}(v(\cdot, t)) \subset \operatorname{supp}(h)
$$

assim as derivadas de $v(\cdot, t)$ também têm suporte contido neste compacto e são nulas fora deste compacto.

Portanto, existe, para cada $j=1, \ldots, m$,

$$
M_{j}=\sup _{\mathbb{R}^{m} \times\{|t| \leq R\}}\left|\frac{\partial v}{\partial x_{j}}\right|,
$$

e então

$$
\left.\mid \nabla_{x} v(x, t)\right) \mid \leq M<\infty,(x, t) \in \mathbb{R}^{m} \times\{|t| \leq R\},
$$

em que

$$
M=\sqrt{m} \max _{1 \leq j \leq m} M_{j}
$$

Da observação acima, concluimos que

$$
\left|v\left(x+\tau^{-1 / 2} x^{\prime}, t\right)-v(x, t)\right| \leq \tau^{-1 / 2} M\left|x^{\prime}\right|, x, x^{\prime} \in \mathbb{R}^{m},|t| \leq R .
$$

Portanto

$$
\left|I_{\tau}(x, t)\right| \leq \tau^{-1 / 2} C
$$


sendo que

$$
C \doteq M \pi^{-m / 2} \int_{\mathbb{R}^{m}}\left|x^{\prime}\right| e^{\frac{-3}{4}\left|x^{\prime}\right|^{2}} d x^{\prime}<\infty,
$$

e consequentemente $I_{\tau}$ converge uniformemente, em $\bar{V}$, para zero quando $\tau \rightarrow+\infty$.

Provemos agora que $J_{\tau}$ também converge uniformemente para zero na mesma região que $I_{\tau}$.

Para cada $K>0$, temos que

$$
\begin{aligned}
J_{\tau}(x, t) & =\pi^{-m / 2} \int_{\left|x^{\prime}\right|<K}\left(e^{-\tau\left[Z(x, t)-Z\left(x+\tau^{-1 / 2} x^{\prime}, t\right)\right]^{2}}-e^{-\left[Z_{x}(x, t) x^{\prime}\right]^{2}}\right) v\left(x+\tau^{-1 / 2} x^{\prime}, t\right) d x^{\prime}+ \\
& +\pi^{-m / 2} \int_{\left|x^{\prime}\right| \geq K}\left(e^{-\tau\left[Z(x, t)-Z\left(x+\tau^{-1 / 2} x^{\prime}, t\right)\right]^{2}}-e^{-\left[Z_{x}(x, t) x^{\prime}\right]^{2}}\right) v\left(x+\tau^{-1 / 2} x^{\prime}, t\right) d x^{\prime},
\end{aligned}
$$

agora vamos estimar cada integral separadamente.

Inicialmente, vamos estimar a integral na região $\left\{\left|x^{\prime}\right| \geq K\right\}$. Visto que

$$
\left|\phi\left(x+\tau^{-1 / 2} x^{\prime}, t\right)-\phi(x, t)\right|=\int_{0}^{1}\left\|\phi_{x}(\lambda(s), t)\right\| \tau^{-1 / 2}\left|x^{\prime}\right| d s \leq \frac{1}{2} \tau^{-1 / 2}\left|x^{\prime}\right|,
$$

então

$$
\begin{aligned}
& \Re \quad-\tau^{1 / 2}\left[Z\left(x+\tau^{-1 / 2} x^{\prime}, t\right)-Z(x, t)\right]^{2}= \\
& =-\tau\left(\tau^{-1 / 2}\left|x^{\prime}\right|^{2}-\left|\phi\left(x+\tau^{-1 / 2} x^{\prime}, t\right)-\phi(x, t)\right|^{2}=\right. \\
& =-\left|x^{\prime}\right|^{2}+\tau\left|\phi\left(x+\tau^{-1 / 2} x^{\prime}, t\right)-\phi(x, t)\right|^{2} \leq \\
& \leq-\frac{3}{4}\left|x^{\prime}\right|^{2} .
\end{aligned}
$$

Logo

$$
\left|e^{-\tau^{1 / 2}\left[Z\left(x+\tau^{-1 / 2} x^{\prime}, t\right)-Z(x, t)\right]^{2}}-e^{-\left[Z_{x}(x, t) x^{\prime}\right]^{2}}\right| \leq 2 e^{-\frac{3}{4}\left|x^{\prime}\right|^{2}},\left|x^{\prime}\right| \leq K .
$$

Por outro lado, $v$ é contínua e tem suporte contido no compacto

$$
\operatorname{supp}(h) \times\{|t| \leq R\}
$$

pois se $x \in \mathbb{R}^{m}$ e $|t| \leq R$, então

$$
v(x, t)=f(x) u(x, t) \operatorname{det} Z_{x}(x, t)=0 .
$$

Sendo assim, existe $C_{v}>0$ tal que

$$
|v(x, t)| \leq C_{v}, \forall(x, t) \in \mathbb{R}^{m} \times\{|s| \leq R\} .
$$

Juntando estes fatos, obtemos que

$$
\begin{array}{cl}
\pi^{-m / 2} & \int_{\left|x^{\prime}\right| \geq K}\left|e^{-\tau\left[Z(x, t)-Z\left(x+\tau^{-1 / 2} x^{\prime}, t\right)\right]^{2}}-e^{-\left[Z_{x}(x, t) x^{\prime}\right]^{2}}\right|\left|v\left(x+\tau^{-1 / 2} x^{\prime}, t\right)\right| d x^{\prime} \leq \\
\leq & \pi^{-m / 2} \int_{\left|x^{\prime}\right| \geq K} 2 e^{-\frac{1}{4}\left|x^{\prime}\right|^{2}} e^{-\frac{1}{2}|K|^{2}} C_{v} d x^{\prime} \leq C e^{-\frac{1}{2}|K|^{2}},
\end{array}
$$


em que

$$
C=2 \pi^{-m / 2} C_{v} \int_{\left|x^{\prime}\right| \geq K} e^{-\frac{1}{4}\left|x^{\prime}\right|^{2}} d x^{\prime}<\infty,
$$

para $|x|,|t| \leq R,\left|x^{\prime}\right| \geq K, \tau>0$.

Para estimarmos a integral do domínio restante, defina

$$
\begin{aligned}
\zeta_{1}(x, t) & \doteq \tau^{1 / 2}\left[Z(x, t)-Z\left(x+\tau^{-1 / 2} x^{\prime}, t\right)\right] \\
\zeta_{2}(x, t) & \doteq-Z_{x}(x, t) x^{\prime}
\end{aligned}
$$

Assim

$$
\begin{aligned}
\zeta_{1}(x, t) & =-\tau^{1 / 2}\left[Z\left(x+\tau^{-1 / 2} x^{\prime}, t\right)-Z(x, t)\right]= \\
& =-\tau^{1 / 2} \int_{0}^{1} Z_{x}(\lambda(s), t) x^{\prime} \tau^{-1 / 2} d s= \\
& =-\int_{0}^{1} Z_{x}(\lambda(s), t) x^{\prime} d s
\end{aligned}
$$

onde

$$
\lambda(s)=x+s \tau^{-1 / 2} x^{\prime} .
$$

Dessa forma tem-se que

$$
\left|\zeta_{1}(x, t)-\zeta_{2}(x, t)\right| \leq \int_{0}^{1}\left\|\phi_{x}\left(x+s \tau^{-1 / 2} x^{\prime}, t\right)-\phi_{x}(x, t)\right\|\left|x^{\prime}\right| d s,
$$

e como $\partial \phi_{j} / \partial x_{j}$ são funções suaves no compacto

$$
\left\{\bar{B}_{R / 2}+\operatorname{supp}(h)\right\} \times \bar{B}_{R},
$$

para cada $j, k=1, \ldots, m$, temos que a diferença 6.13 converge para zero uniformemente quando $\tau \rightarrow+\infty$.

Além disso, observe que

$$
\begin{aligned}
\Re\left[\zeta_{1}(x, t)\right]^{2} & =\Re\left[\left|x^{\prime}\right|+i \tau^{1 / 2}\left(\phi(x, t)-\phi\left(x+\tau^{-1 / 2} x^{\prime}, t\right)\right)\right]^{2}= \\
& =\left|x^{\prime}\right|^{2}-\tau\left|\phi(x, t)-\phi\left(x+\tau^{-1 / 2} x^{\prime}, t\right)\right|^{2} \geq \\
& \geq\left|x^{\prime}\right|^{2}-\frac{1}{4}\left|x^{\prime}\right|^{2}=\frac{3}{4}\left|x^{\prime}\right|^{2} \geq 0 .
\end{aligned}
$$

Destes dois fatos, obtemos que para $\tau$ suficientemente grande $\Re\left[\zeta_{2}(x, t)\right]^{2} \geq 0$.

Observação 6.4.20. Note que a função inteira $f(\zeta)=e^{-\zeta}, \zeta \in \mathbb{C}$ é lipschitziana em $[0,+\infty)+i \mathbb{R}$, ou seja, existe constante $M_{0}$ tal que

$$
|f(\xi)-f(\zeta)| \leq M_{0}|\xi-\zeta|
$$

para quaisquer $\zeta, \xi$ números complexos com parte real maior ou igual a zero. 
Pela observação acima concluimos que

$$
\begin{aligned}
\left|e^{-\tau\left[Z(x, t)-Z\left(x+\tau^{-1 / 2} x^{\prime}, t\right)\right]^{2}}-e^{-\left[Z_{x}(x, t) x^{\prime}\right]^{2}}\right| & =\left|e^{-\left[\left[\zeta_{1}(x, t)\right]^{2}\right.}-e^{-\left[\zeta_{2}(x, t)\right]^{2}}\right| \leq \\
& \leq M_{0}\left|\left[\zeta_{1}(x, t)\right]^{2}-\left[\zeta_{2}(x, t)\right]^{2}\right|
\end{aligned}
$$

Sendo assim, vamos estimar agora as partes real e imaginária da diferença

$$
\left|\left[\zeta_{1}(x, t)\right]^{2}-\left[\zeta_{2}(x, t)\right]^{2}\right| .
$$

Começamos pela parte imaginária:

$$
\begin{gathered}
\left|\Im\left(\left[\zeta_{1}(x, t)\right]^{2}-\left[\zeta_{2}(x, t)\right]^{2}\right)\right|= \\
\left|2 i \sum_{j=1}^{m} x_{j}^{\prime}\left(-\tau^{1 / 2}\left(\phi_{j}\left(x+\tau^{1 / 2} x^{\prime}, t\right)-\phi_{j}(x, t)-\nabla_{x} \phi_{j}(x, t) \tau^{-1 / 2} x^{\prime}\right)\right)\right| .
\end{gathered}
$$

Sabemos também que

$$
\begin{gathered}
\left|\left(\phi_{j}\left(x+\tau^{1 / 2} x^{\prime}, t\right)-\phi_{j}(x, t)-\nabla_{x} \phi_{j}(x, t) \tau^{-1 / 2} x^{\prime}\right)\right| \leq \\
\leq \int_{0}^{1}\left\|\nabla_{x} \phi_{j}\left(x+s \tau^{-1 / 2} x^{\prime}, t\right)-\nabla_{x} \phi_{j}(x, t)\right\| \tau^{-1 / 2}\left|x^{\prime}\right| d s \leq \\
\frac{m^{1 / 2} M \tau^{-1}\left|x^{\prime}\right|}{2}
\end{gathered}
$$

em que

$$
M=\max _{1 \leq j, k \leq m} \sup _{\left\{\bar{B}_{R / 2}+\operatorname{supp}(h)\right\} \times \bar{B}_{R}}\left\|\nabla_{x}\left(\frac{\partial \phi_{j}}{\partial e_{k}}\right)\right\| .
$$

Logo

$$
\left|\Im\left(\left[\zeta_{1}(x, t)\right]^{2}-\left[\zeta_{2}(x, t)\right]^{2}\right)\right| \leq m^{3 / 2} M \tau^{-1 / 2}\left|x^{\prime}\right|^{2} .
$$

Resta estimar a parte real

$$
\begin{array}{r}
\left|\Re\left(\left[\zeta_{1}(x, t)\right]^{2}-\left[\zeta_{2}(x, t)\right]^{2}\right)\right|=|-\tau| \phi\left(x+\tau^{1 / 2} x^{\prime}, t\right)-\left.\phi(x, t)\right|^{2}+\left|\phi_{x}(x, t) x^{\prime}\right|^{2} \mid= \\
\left|-\tau\left(\sum_{j=1}^{m}\left(\phi_{j}\left(x+\tau^{-1 / 2} x^{\prime}, t\right)-\phi_{j}(x, t)\right)^{2}-\left(\nabla_{x} \phi_{j}(x, t) \tau^{-1 / 2} x^{\prime}\right)^{2}\right)\right|
\end{array}
$$

Mas, temos que $\left(\phi_{j}\left(x+\tau^{1 / 2} x^{\prime}, t\right)-\phi_{j}(x, t)\right)^{2}=$

$$
\begin{aligned}
& =\left(\phi_{j}\left(x+\tau^{-1 / 2} x^{\prime}, t\right)-\phi_{j}(x, t)+\nabla_{x} \phi_{j}(x, t) \tau^{-1 / 2} x^{\prime}-\nabla_{x} \phi_{j}(x, t) \tau^{-1 / 2} x^{\prime}\right)^{2}= \\
& =\left(\phi_{j}\left(x+\tau^{-1 / 2} x^{\prime}, t\right)-\phi_{j}(x, t)-\nabla_{x} \phi_{j}(x, t) \tau^{-1 / 2} x^{\prime}\right)^{2}- \\
& -2\left(\phi_{j}\left(x+\tau^{-1 / 2} x^{\prime}, t\right)-\phi_{j}(x, t)+\nabla_{x} \phi_{j}(x, t) \tau^{-1 / 2} x^{\prime}\right) \nabla_{x} \phi_{j}(x, t) \tau^{-1 / 2} x^{\prime}+ \\
& +\left(\nabla_{x} \phi_{j}(x, t) \tau^{-1 / 2} x^{\prime}\right)^{2}
\end{aligned}
$$

então $\left|\Re\left(\left[\zeta_{1}(x, t)\right]^{2}-\left[\zeta_{2}(x, t)\right]^{2}\right)\right|$ é dada pela expressão

$$
\mid-\tau \sum_{j=1}^{m}\left(\left|\phi_{j}\left(x+\tau^{1 / 2} x^{\prime}, t\right)-\phi_{j}(x, t)-\nabla_{x} \phi_{j}(x, t) \tau^{-1 / 2} x^{\prime}\right|^{2}-\right.
$$




$$
\left.-2\left(\phi_{j}\left(x+\tau^{-1 / 2} x^{\prime}, t\right)-\phi_{j}(x, t)+\nabla_{x} \phi_{j}(x, t) \tau^{-1 / 2} x^{\prime}\right) \nabla_{x} \phi_{j}(x, t) \tau^{-1 / 2} x^{\prime}\right) \mid
$$

e consequentemente

$$
\left|\Re\left(\left[\zeta_{1}(x, t)\right]^{2}-\left[\zeta_{2}(x, t)\right]^{2}\right)\right| \leq \tau \sum_{j=1}^{m}\left(\frac{m M^{2}\left|x^{\prime}\right|^{4} \tau^{-2}}{4}+2 C_{0} \frac{m^{1 / 2} M\left|x^{\prime}\right|^{3} \tau^{-3 / 2}}{2}\right) .
$$

Portanto

$$
\left|e^{-\tau\left[Z(x, t)-Z\left(x+\tau^{-1 / 2} x^{\prime}, t\right)\right]^{2}}-e^{-\left[Z_{x}(x, t) x^{\prime}\right]^{2}}\right| \leq \hat{C} \tau^{-1 / 2}
$$

e então

$$
\begin{aligned}
\pi^{-m / 2} & \int_{\left|x^{\prime}\right| \leq K}\left|e^{-\tau\left[Z(x, t)-Z\left(x+\tau^{-1 / 2} x^{\prime}, t\right)\right]^{2}}-e^{-\left[Z_{x}(x, t) x^{\prime}\right]^{2}}\right|\left|v\left(x+\tau^{-1 / 2} x^{\prime}, t\right)\right| d x^{\prime} \leq \\
\leq & C K^{m} \tau^{-1 / 2} .
\end{aligned}
$$

Em suma, provamos que existe constante $C_{J}$ tal que

$$
\left|J_{\tau}(x, t)\right| \leq C_{J}\left(K^{m} \tau^{-1 / 2}+e^{-K / 2}\right), K, \tau>0 .
$$

Fazendo $\tau \rightarrow+\infty$ obtemos que

$$
\varlimsup_{\tau \rightarrow \infty}\left|J_{\tau}(x, t)\right| \leq C_{J} e^{-K / 2}, K>0
$$

finalmente fazendo $K \rightarrow+\infty$ concluimos que $J_{\tau}$ converge para 0 em $\bar{V}$.

Observação 6.4.21. Observe que a partir da proposição acima que quando $\tau \rightarrow+\infty$ acontecem as seguintes convergências:

$$
\begin{aligned}
G_{\tau} u & =\left(\frac{\tau}{\pi}\right)^{m / 2} H_{\tau h} u \rightarrow h u, \\
{\left[M_{k}, G_{\tau}\right] u } & =\left(\frac{\tau}{\pi}\right)^{m / 2} H_{\tau M_{k} h} u \rightarrow M_{k}(h) u, \\
{\left[L_{j}, G_{\tau}\right] u } & =\left(\frac{\tau}{\pi}\right)^{m / 2} H_{\tau L_{j} h} u \rightarrow L_{j}(h) u,
\end{aligned}
$$

e como $h(x)=1,|x| \leq R / 2$ então

$$
\begin{aligned}
M_{k} G_{\tau} u & =\left[M_{k}, G_{\tau}\right] u+G_{\tau} M_{k} u \rightarrow M_{k}(h) u+M_{k} u=M_{k} u, \\
L_{j} G_{\tau} u & =\left[L_{j}, G_{\tau}\right] u+G_{\tau} L_{j} u \rightarrow L_{j}(h) u+L_{j} u=L_{j} u,
\end{aligned}
$$

quando $\tau \rightarrow+\infty$ no conjunto

$$
U \doteq\{(x, t) ;|x| \leq R / 2,|t| \leq R\}
$$

Estes fatos irão nos ajudar a demonstrar que $G_{\tau}$ u converge para u na topologia de $C^{\infty}(U)$. 
Além disso,

$$
\begin{aligned}
L_{j}(\tau / \pi)^{m / 2} H_{\tau f} u & =(\tau / \pi)^{m / 2}\left(H_{\tau L_{j} f} u+H_{\tau f} L_{j} u\right) \rightarrow L_{j}(f) u+f L_{j} u, \\
M_{k}(\tau / \pi)^{m / 2} H_{\tau f} u & =(\tau / \pi)^{m / 2}\left(H_{\tau M_{k} f} u+H_{\tau f} M_{k} u\right) \rightarrow M_{k}(f) u+f M_{k} u,
\end{aligned}
$$

quando $\tau \rightarrow+\infty$.

O seguinte resultado é uma generalização da observação acima para quaisquer quantidades finitas de campos $L_{j}, M_{k}$. Antes de tal resultado, renomeie os campos da seguinte maneira

$$
\begin{aligned}
& L_{j}^{\#} \doteq L_{j}, j=1, \ldots, n, \\
& L_{j}^{\#} \doteq L_{j-n}, j=n+1, \ldots, N .
\end{aligned}
$$

Proposição 6.4.22. Seja $u \in C^{\infty}(W)$, então quando $\tau$ vai para infinito temos as convergências:

$$
L_{J}^{\#}(\tau / \pi)^{m / 2} H_{\tau L_{K}^{\#} h} u \rightarrow 0
$$

$e$

$$
L_{J}^{\#}\left[L_{j_{0}}^{\#}, G_{\tau}\right] u \rightarrow 0
$$

no conjunto $U$, onde

$$
L_{J}^{\#} \doteq L_{j_{1}}^{\#} \ldots L_{j_{r}}^{\#}
$$

$e$

$$
L_{K}^{\#} \doteq L_{k_{1}}^{\#} \ldots L_{k_{s}}^{\#}
$$

são quaiquer quantidades de campos.

Demonstração. Primeiramente, destacamos que $L_{K}^{\#} h$ é uma função suave com suporte compacto contido no suporte de $h$ e como $h$ é identicamente 1 numa vizinhança de $U$, esta função se anula em $U$.

Faremos a prova por indução. Da Observação 6.4.21 tiramos o caso inicial.

Suponha 6.14 válida para $L_{J}^{\#}$ e provemos para $L_{j_{0}}^{\#} L_{J}^{\#}$. Basta notar que como os campos são comutativos entre si, vale

$$
\begin{aligned}
L_{j_{0}}^{\#} L_{J}^{\#}(\tau / \pi)^{m / 2} H_{\tau L_{K}^{\#} h} u & =L_{j_{0}}^{\#} L_{j_{1}}^{\#} \ldots L_{j_{r}}^{\#}(\tau / \pi)^{m / 2} H_{\tau L_{K}^{\#} h} u= \\
& =L_{j_{1}}^{\#} \ldots L_{j_{r}}^{\#}(\tau / \pi)^{m / 2} L_{j_{0}}^{\#} H_{\tau L_{K}^{\#} h} u= \\
& =L_{j_{1}}^{\#} \ldots L_{j_{r}}^{\#}(\tau / \pi)^{m / 2}\left(H_{\tau L_{j_{0}}^{\#} L_{K}^{\#} h} u+H_{\tau L_{K}^{\#} h} L_{j_{0}}^{\#} u\right),
\end{aligned}
$$

pela hipótese de indução concluimos a convergência.

Resta mostrar que

$$
L_{J}^{\#}\left[L_{j_{0}}^{\#}, G_{\tau}\right] u \rightarrow 0
$$


O caso inicial já foi provado anteriormente, suponha válida a convergência acima e provemos para

$$
L_{k_{0}} L_{J}^{\#}\left[L_{j_{0}}^{\#}, G_{\tau}\right] u \rightarrow 0
$$

Como

$$
\left[L_{j_{0}}^{\#}, G_{\tau}\right] u=(\tau / \pi)^{m / 2} H_{\tau L_{j_{0}}^{\#} h} u
$$

então

$$
L_{k_{0}} L_{J}^{\#}\left[L_{j_{0}}^{\#}, G_{\tau}\right] u=L_{k_{0}} L_{J}^{\#}(\tau / \pi)^{m / 2} H_{\tau L_{j_{0}}^{\#} h} u
$$

e usando a convergência de 6.14 finalizamos a demonstração.

Ressaltamos que de 6.4.14 temos, voltando a notação original dos campos,

$$
L_{J} M_{K} G_{\tau} u=\left[L_{j_{1}}, G_{\tau}\right] L_{j_{2}} \ldots M_{k_{r}} u+\ldots+L_{j_{1}} \ldots M_{k_{r-1}}\left[M_{k_{r}}, G_{\tau}\right] u+G_{\tau} L_{J} M_{K} u
$$

e combinando com 6.4.22 obtemos que

$$
L_{J} M_{K} G_{\tau} u \rightarrow L_{J} M_{K} u
$$

em $U$ quando $\tau \rightarrow+\infty$.

O próximo resultado nos fornecerá uma forma de escrever qualquer derivada

$$
\partial^{\alpha} \doteq \partial^{\alpha_{1}} \ldots \partial^{\alpha_{k}}
$$

como combinação de composições dos campos $L_{j}^{\#}$, em que os coeficientes são funções suaves. Assim seremos capazes de obter que $G_{\tau} u$ converge para $u$ na topologia de $C^{\infty}(U)$ quando $\tau \rightarrow+\infty$.

Proposição 6.4.23. Seja $\alpha \in \mathbb{Z}^{N}$ multi-índice, então existem funções suaves

$$
a_{j_{1}}, a_{j_{1}, j_{2}}, \ldots, a_{j_{1}, \ldots, j_{|\alpha|}},
$$

onde $|\alpha|$ é comprimento do multi-índice, tais que

$$
\begin{aligned}
\partial^{\alpha} & =\sum_{j_{1}, \ldots, j_{|\alpha|}=1}^{N} a_{j_{1}, \ldots, j_{|\alpha|}} L_{j_{1}}^{\#} \ldots L_{j_{|\alpha|}}^{\#}+\sum_{j_{1}, \ldots, j_{|\alpha|-1}=1}^{N} a_{j_{1}, \ldots, j_{|\alpha|-1}} L_{j_{1}}^{\#} \ldots L_{j_{|\alpha|-1}}^{\#}+ \\
& +\sum_{j_{1}, j_{2}=1}^{N} a_{j_{1}, j_{2}} L_{j_{1}}^{\#} L_{j_{2}}^{\#}+\sum_{j_{1}=1}^{N} a_{j_{1}} L_{j_{1}}^{\#} .
\end{aligned}
$$

Demonstração. Novamente, faremos a prova por indução. Observe que o caso inicial é consequência de $\left\{L_{1}^{\#}, \ldots, L_{N}^{\#}\right\}$ ser um conjunto linearmente independente e então geram qualquer campo, ou seja, como $\partial_{k} \in \mathfrak{X}(U)$ então existem funções suaves $a_{1}, \ldots, a_{N}$ tais que

$$
\partial_{k}=\sum_{j=1}^{N} a_{j} L_{j}^{\#} .
$$


Suponha válida a fórmula da proposição para multi-índices de comprimento $|\alpha|=$ $k_{0}$ e provemos para multi-índices de comprimento $k_{0}+1$.

$$
\begin{aligned}
\partial_{k} \partial^{\alpha} & =\sum_{j=1}^{N} a_{j} L_{j}^{\#}\left(\partial^{\alpha}\right)=\sum_{j, j_{1}, \ldots, j_{|\alpha|}=1}^{N} a_{j} a_{j_{1}, \ldots, j_{|\alpha|}} L_{j}^{\#} L_{j_{1}}^{\#} \ldots L_{j_{|\alpha|}}^{\#}+ \\
& +\sum_{j_{1}, \ldots, j_{|\alpha|}=1}^{N}\left(\sum_{j=1}^{N} a_{j} L_{j}^{\#}\left(a_{j_{1}, \ldots, j_{|\alpha|}}\right)\right) a_{j_{1}, \ldots, j_{|\alpha|}} L_{j_{1}}^{\#} \ldots L_{j_{|\alpha|}}^{\#}+ \\
& +\sum_{j, j_{1}, \ldots, j_{|\alpha|-1}=1}^{N} a_{j} a_{j_{1}, \ldots, j_{|\alpha|-1}} L_{j}^{\#} L_{j_{1}}^{\#} \ldots L_{j_{|\alpha|-1}}^{\#}+\ldots+\sum_{j, j_{1}=1}^{N} a_{j} a_{j_{1}} L_{j}^{\#} L_{j_{1}}^{\#}+ \\
& +\sum_{j_{1}=1}^{N}\left(\sum_{j=1}^{N} a_{j} L_{j}^{\#}\left(a_{\left.j_{1}\right)}\right) L_{j_{1}}^{\#}=\sum_{j_{1}, \ldots, j_{|\alpha|}=1}^{N} \tilde{a}_{j_{1}, \ldots, j_{|\alpha|}} L_{j_{1}}^{\#} \ldots L_{j_{|\alpha|}}^{\#}+\sum_{j_{1}=1}^{N} \tilde{a}_{j_{1}} L_{j_{1}}^{\#},\right.
\end{aligned}
$$

em que

$$
\begin{gathered}
\tilde{a}_{j_{1}, \ldots, j_{l}}=a_{j_{l}} a_{j_{1}, \ldots, j_{l-1}}+\sum_{l=1}^{N} a_{l} L_{l}^{\#}\left(a_{j_{1}, \ldots, j_{k-1}}\right), l=2, \ldots, k_{0}, \\
\tilde{a}_{j_{1}, \ldots, j_{k_{0}+1}}=a_{j_{k_{0}+1}} a_{j_{1}, \ldots, j_{k_{0}}}, \\
\tilde{a}_{j_{1}}=\sum_{j=1}^{N} a_{j} L_{j}^{\#}\left(a_{j_{1}}\right)
\end{gathered}
$$

finalizando a demonstração

Teorema 6.4.24. Seja $u \in C^{\infty}(W)$, então $G_{\tau} u$ converge para $u$, na topologia de $C^{\infty}(U)$, quando $\tau \rightarrow+\infty$.

Demonstração. Como já foi feito que $G_{\tau} u$ converge para $u$ uniformemente em $U$, resta mostrar que $\partial^{\alpha} G_{\tau} u$ converge uniformemente para $\partial^{\alpha} u$ em $U$, para qualquer multi-índice $\alpha \in \mathbb{Z}^{N}$.

Primeiramente, observe que pela proposição anterior vale

$$
\partial^{\alpha} G_{\tau} u=\sum_{j_{1}, \ldots, j_{|\alpha|}=1}^{N} a_{j_{1}, \ldots, j_{|\alpha|}} L_{j_{1}}^{\#} \ldots L_{j_{|\alpha|}}^{\#} G_{\tau} u+\ldots+\sum_{j_{1}=1}^{N} a_{j_{1}} L_{j_{1}}^{\#} G_{\tau} u,
$$

que converge, em $U$ quando $\tau \rightarrow+\infty$, para

$$
\sum_{j_{1}, \ldots, j_{|\alpha|}=1}^{N} a_{j_{1}, \ldots, j_{|\alpha|}} L_{j_{1}}^{\#} \ldots L_{j_{|\alpha|}}^{\#} u+\ldots+\sum_{j_{1}=1}^{N} a_{j_{1}} L_{j_{1}}^{\#} u=\partial^{\alpha} u
$$

como queríamos demonstrar. 



\section{Fórmula de Aproximação de Baouendi-}

\section{Treves}

Neste capítulo provaremos um dos resultados mais importantes envolvendo a teoria de estruturas localmente integráveis. O Teorema de Baouendi-Treves afirma que dada uma estrutura localmente integrável $\mathcal{L}$ sobre $\Omega$, então qualquer solução da equação $\mathcal{L} u=0$ pode ser aproximada, localmente, por polinômios nas suas integrais primeiras.

A prova de tal resultado para o caso distribucional exige o conhecimento de conceitos mais sofisticados como alguns tópicos da Teoria das Distribuições e de Análise Funcional.

\subsection{Versão Clássica}

Antes de apresentarmos o enunciado e a demonstração do teorema vamos apresentar um exemplo de um caso mais particular e bem conhecido da análise complexa em que podemos aplicar o Teorema de Baouendi-Treves.

Exemplo 7.1.1. Seja $\Omega \subset \mathbb{R}^{2}$ um aberto. Considere $\mathcal{L}=\cup_{p \in \Omega} \mathcal{L}_{p}$, em que cada fibra $\mathcal{L}_{p}$ é gerada pelo operador de Cauchy-Riemann

$$
L=\frac{1}{2}\left(\frac{\partial}{\partial x}+i \frac{\partial}{\partial y}\right) .
$$

Observe que $\mathcal{L}$ é uma estrutura localmente integrável unidimensional, pois as diferenciais da função $Z=x+$ iy calculadas em $p \in \Omega$ geram as fibras de $\mathcal{L}^{\perp}$. Além disso, se $u \in C^{\infty}(\Omega)$ é uma solução de $\mathcal{L} u=0$, temos que

$$
\frac{1}{2}\left(\frac{\partial}{\partial x}+i \frac{\partial}{\partial y}\right) u=0
$$

e da análise complexa sabemos que u é uma função holomorfa, portanto esta função admite representação em série de potências em torno de cada ponto $p \in \Omega$, ou seja, u é limite de polinômios holomorfos na variável $Z$.

Visto que a fórmula de aproximação possui natureza local, é suficiente focarmos nossa atenção no caso em que $\mathcal{L}$ é uma estrutura localmente integrável sobre um aberto $\Omega \subset \mathbb{R}^{N}$ que contém a origem, em que

$$
\mathcal{L}_{p}^{\perp}=\left[d Z_{1 p}, \ldots, d Z_{m p}\right], \forall p \in \Omega
$$

e $Z_{1}, \ldots, Z_{m} \in C^{\infty}(\Omega)$. 
Teorema 7.1.2 (Fórmula de Baouendi-Treves). Sejam $\Omega \subset \mathbb{R}^{N}$ um aberto que contém a origem e $\mathcal{L}$ estrutura localmente integrável sobre $\Omega$, em que

$$
\mathcal{L}_{p}^{\perp}=\left[d Z_{1 p}, \ldots, d Z_{m p}\right], \forall p \in \Omega
$$

e $Z_{1}, \ldots, Z_{m} \in C^{\infty}(\Omega)$. Nessas condições, existem abertos $U$ e $W$ que contém a origem, $U \subset \bar{U} \subset W \subset \Omega$ tais que se $u \in C^{\infty}(W)$ satisfaz

$$
\mathcal{L} u=0,
$$

então existe uma sequência de polinômios $P_{l} \circ Z=P_{l}\left(Z_{1}, \ldots, Z_{m}\right)$ de forma que

$$
\lim _{l \rightarrow \infty} d_{C^{\infty}(U)}\left(u, P_{l} \circ Z\right)=0 .
$$

Demonstração. Para a prova, defina

$$
\begin{aligned}
E_{\tau} u(x, t) & \doteq\left(\frac{\tau}{\pi}\right)^{m / 2} \int_{\mathbb{R}^{m}} e^{-\tau\left[Z(x, t)-Z\left(x^{\prime}, 0\right]^{2}\right.} u\left(x^{\prime}, 0\right) h\left(x^{\prime}\right) \operatorname{det} Z_{x}\left(x^{\prime}, 0\right) d x^{\prime} \\
R_{\tau} u(x, t) & \doteq G_{\tau} u(x, t)-E_{\tau} u(x, t)
\end{aligned}
$$

mostremos que $R_{\tau} u$ converge uniformemente para zero em $U$ quando $\tau \rightarrow+\infty$ e por fim aproximemos a função $e^{-\tau[\zeta]^{2}}$ na topologia de $C^{\infty}$, para $\tau$ fixado suficientemente grande, pela soma parcial, de grau $l, P_{l}(\zeta)$ da sua série de Taylor em

$$
\left\{\tau^{1 / 2}\left(Z(x, t)-Z\left(x^{\prime}, 0\right)\right) ;|x|,\left|x^{\prime}\right| \leq R / 2,|t| \leq R\right\} .
$$

Assim teremos

$$
P_{l}\left(Z(x, t)-Z\left(x^{\prime}, 0\right)\right)=\sum_{|\alpha|=0}^{l} C_{\alpha}\left(x^{\prime}\right)(Z(x, t))^{\alpha},
$$

em que $C_{\alpha}\left(x^{\prime}\right)$ são funções suaves e então

$$
\begin{aligned}
& d_{C^{\infty}(U)}\left(u(\cdot),(\tau / \pi)^{m / 2} \sum_{|\alpha|=0}^{l}(Z(\cdot))^{\alpha} \int_{\mathbb{R}^{m}} C_{\alpha}\left(x^{\prime}\right) h\left(x^{\prime}\right) \operatorname{det} Z_{x}\left(x^{\prime}, 0\right) d x^{\prime}\right) \leq \\
\leq & d_{C^{\infty}(U)}\left(u(\cdot), G_{\tau}(\cdot)\right)+d_{C^{\infty}(U)}\left(R_{\tau} u(\cdot), 0\right)+ \\
+ & d_{C^{\infty}(U)}\left(E_{\tau} u(\cdot),(\tau / \pi)^{m / 2} \int_{\mathbb{R}^{m}} P_{l}\left(Z(\cdot)-Z\left(x^{\prime}, 0\right)\right) h\left(x^{\prime}\right) \operatorname{det} Z_{x}\left(x^{\prime}, 0\right) d x^{\prime}\right),
\end{aligned}
$$

em que

$$
d_{C^{\infty}(U)}\left(f_{1}, f_{2}\right), f_{1}, f_{2} \in C^{\infty}(U)
$$

representa a distância na topologia de $C^{\infty}(U)$ (Confira [3]).

Portanto, fazendo $l \rightarrow \infty$

$$
\begin{array}{r}
\lim _{l \rightarrow \infty} d_{C^{\infty}(U)}\left(u(\cdot),(\tau / \pi)^{m / 2} \sum_{|\alpha|=0}^{l}(Z(\cdot))^{\alpha} \int_{\mathbb{R}^{m}} C_{\alpha}\left(x^{\prime}\right) h\left(x^{\prime}\right) \operatorname{det} Z_{x}\left(x^{\prime}, 0\right) d x^{\prime}\right) \leq \\
\leq d_{C^{\infty}(U)}\left(u(\cdot), G_{\tau}(\cdot)\right)+d_{C^{\infty}(U)}\left(R_{\tau} u(\cdot), 0\right),
\end{array}
$$


para cada $\tau$ suficientemente grande fixado.

Por fim, fazendo $\tau \rightarrow+\infty$ :

$$
\lim _{l \rightarrow+\infty} d_{C^{\infty}(U)}\left(u(\cdot),(\tau / \pi)^{m / 2} \sum_{|\alpha|=0}^{\infty}(Z(\cdot))^{\alpha} \int_{\mathbb{R}^{m}} C_{\alpha}\left(x^{\prime}\right) h\left(x^{\prime}\right) \operatorname{det} Z_{x}\left(x^{\prime}, 0\right) d x^{\prime}\right)=0 .
$$

Para estimarmos o termo $R_{\tau} u$ fazemos uso do Teorema de Stokes. Considere a $m$-forma

$$
\omega\left(x^{\prime}, t^{\prime}\right) \doteq v\left(x^{\prime}, t^{\prime}\right) d Z\left(x^{\prime}, t^{\prime}\right)
$$

em que

$$
v\left(x^{\prime}, t^{\prime}\right)=(\tau / \pi)^{m / 2} e^{-\tau\left[Z(x, t)-Z\left(x^{\prime}, t^{\prime}\right)\right]^{2}} h\left(x^{\prime}\right) u\left(x^{\prime}, t^{\prime}\right)
$$

e

$$
d Z\left(x^{\prime}, t^{\prime}\right)=d Z_{1}\left(x^{\prime}, t^{\prime}\right) \wedge \ldots \wedge d Z_{m}\left(x^{\prime}, t^{\prime}\right)
$$

Nessas condições, temos que

$$
\begin{aligned}
G_{\tau} u(x, t) & =(\tau / \pi)^{m / 2} \int_{\mathbb{R}^{m}} e^{-\tau\left[Z(x, t)-Z\left(x^{\prime}, t^{\prime}\right)\right]^{2}} u\left(x^{\prime}, t\right) h\left(x^{\prime}\right) \operatorname{det} Z_{x}\left(x^{\prime}, t\right) d x^{\prime}= \\
& =\int_{\mathbb{R}^{m}} i_{t}^{*} \omega d x^{\prime}=\int_{i_{t}\left(\mathbb{R}^{m}\right)} \omega=\int_{\mathbb{R}^{m} \times\{t\}} \omega,
\end{aligned}
$$

em que $i_{t}^{*} \omega$ é o pull-back de $\omega$ pela aplicação

$$
i_{t}\left(x^{\prime}\right)=\left(x^{\prime}, t\right), x^{\prime} \in \mathbb{R}^{m} .
$$

Analogamente obtemos que

$$
E_{\tau} u(x, t)=\int_{\mathbb{R}^{m} \times\{0\}} \omega .
$$

Pelo Teorema de Stokes concluímos que

$$
R_{\tau} u(x, t)=\int_{\mathbb{R}^{m} \times\{t\}} \omega-\int_{\mathbb{R}^{m} \times\{0\}} \omega=\int_{\mathbb{R}^{m} \times[0, t]} d \omega .
$$

Como $\omega=v d Z$ temos

$$
d \omega=d v \wedge d Z+v \wedge d(d Z)=d v \wedge d Z .
$$

Além do mais, visto que

$$
d v=\sum_{j=1}^{n} L_{j}(v) d t_{j}+\sum_{k=1}^{m} M_{k}(v) d Z_{k}
$$

obtemos que

$$
d \omega=\sum_{j=1}^{n} L_{j}(v) d t_{j} \wedge d Z+\sum_{k=1}^{m} M_{k}(v) d Z_{k} \wedge d Z=\sum_{j=1}^{n} L_{j}(v) d t_{j} \wedge d Z .
$$


Por outro lado,

$$
\begin{aligned}
L_{j} v\left(x^{\prime}, t^{\prime}\right) & =(\tau / \pi)^{m / 2} L_{j}\left(e^{-\tau[Z(x, t)-Z(\cdot)]^{2}} h(\cdot) u(\cdot)\right)\left(x^{\prime}, t^{\prime}\right)= \\
& =(\tau / \pi)^{m / 2} L_{j}\left(e^{-\tau[Z(x, t)-Z(\cdot)]^{2}}\right)\left(x^{\prime}, t^{\prime}\right) h\left(x^{\prime}\right) u\left(x^{\prime}, t^{\prime}\right)+ \\
& +(\tau / \pi)^{m / 2}\left(e^{-\tau\left[Z(x, t)-Z\left(x^{\prime}, t^{\prime}\right)\right]^{2}}\right) L_{j}(h)\left(x^{\prime}\right) u\left(x^{\prime}, t^{\prime}\right) \\
& +(\tau / \pi)^{m / 2}\left(e^{-\tau\left[Z(x, t)-Z\left(x^{\prime}, t^{\prime}\right)\right]^{2}}\right) h\left(x^{\prime}\right) L_{j}(u)\left(x^{\prime}, t^{\prime}\right)= \\
& =(\tau / \pi)^{m / 2}\left(e^{-\tau\left[Z(x, t)-Z\left(x^{\prime}, t^{\prime}\right)\right]^{2}}\right) L_{j}(h)\left(x^{\prime}\right) u\left(x^{\prime}, t^{\prime}\right),
\end{aligned}
$$

pois

$$
L_{j} u=L_{j} Z_{k}=0
$$

Reunindo os fatos acima, tem-se que

$$
R_{\tau} u(x, t)=(\tau / \pi)^{m / 2} \int_{\mathbb{R}^{m} \times[0, t]} \sum_{j=1}^{n}\left(e^{-\tau\left[Z(x, t)-Z\left(x^{\prime}, t^{\prime}\right)\right]^{2}}\right) L_{j}(h)\left(x^{\prime}\right) u\left(x^{\prime}, t^{\prime}\right) d t_{j} \wedge d Z\left(x^{\prime}, t^{\prime}\right) .
$$

Para concluirmos a convergência do resto $R_{\tau} u$ basta estimarmos o integrando acima.

Primeiramente, note que

$$
\begin{aligned}
\Re\left(-\tau\left[Z(x, t)-Z\left(x^{\prime}, t^{\prime}\right)\right]^{2}\right) & =\Re\left(-\tau\left[x-x^{\prime}-i\left(\phi(x, t)-\phi\left(x^{\prime}, t^{\prime}\right)\right)\right]^{2}\right)= \\
& =-\tau\left|x-x^{\prime}\right|^{2}+\tau\left|\phi(x, t)-\phi\left(x^{\prime}, t^{\prime}\right)\right|^{2} .
\end{aligned}
$$

Assim

$$
\left|\phi(x, t)-\phi\left(x^{\prime}, t^{\prime}\right)\right| \leq\left|\phi(x, t)-\phi\left(x, t^{\prime}\right)\right|+\left|\phi\left(x, t^{\prime}\right)-\phi\left(x^{\prime}, t^{\prime}\right)\right| \leq \frac{1}{2}\left|x-x^{\prime}\right|+C\left|t-t^{\prime}\right|,
$$

em que

$$
C=\max _{1 \leq j \leq m} \sup _{\bar{B}_{R / 2} \times \bar{B}_{3 R / 2}}\left\|\nabla_{t} \phi_{j}(x, t)\right\| .
$$

Então

$$
\begin{aligned}
\left|\phi(x, t)-\phi\left(x^{\prime}, t^{\prime}\right)\right|^{2} & \leq \frac{1}{4}\left|x-x^{\prime}\right|^{2}+C \frac{R}{2}+C^{2} \frac{R^{2}}{4}= \\
& =\frac{1}{2}\left|x-x^{\prime}\right|^{2}+\left(\frac{-1}{R^{2}}\left|x-x^{\prime}\right|^{2}+\frac{2 C}{R}\left|x-x^{\prime}\right| C^{2}\right) \frac{R^{2}}{4} \leq \\
& \leq \frac{1}{2}\left|x-x^{\prime}\right|^{2}+\lambda \frac{R^{2}}{2}
\end{aligned}
$$

para $\lambda \in \mathbb{R}$ tal que

$$
\lambda \geq \frac{5 C}{4}+\frac{C^{2}}{2} .
$$

Além disso, como

$$
\operatorname{supp}\left(L_{j}(h)\right) \subset \operatorname{supp}(h) \subset \bar{B}_{R},
$$


e $h$ é identicamente 1 em $\bar{B}_{R / 2}$ então $L_{j}(h)$ se anula em

$$
\left\{x^{\prime} \in \mathbb{R}^{m} ;\left|x^{\prime}\right|>R\right\} \cup\left\{x^{\prime} \in \mathbb{R}^{m} ;\left|x^{\prime}\right| \leq R / 2\right\},
$$

$\operatorname{logo}$ para $R / 2 \leq\left|x^{\prime}\right| \leq R$ temos

$$
\left|e^{-\tau\left[Z(x, t)-Z\left(x^{\prime}, t^{\prime}\right)\right]^{2}}\right| \leq e^{\tau\left(\frac{1}{2}\left|x-x^{\prime}\right|^{2}+2 \lambda \frac{R^{2}}{4}-\left|x-x^{\prime}\right|^{2}\right)} \leq e^{\tau\left(\lambda \frac{R^{2}}{2} \frac{-1}{2}\left|x-x^{\prime}\right|^{2}\right)} \leq e^{\tau\left(\lambda \frac{R^{2}}{2} \frac{-R^{2}}{32}\right)} \leq e^{\frac{-\tau R^{2}}{33}}
$$

para $R>0$ suficientemente pequeno, ou seja, se necessário podemos diminuir a vizinhança da origem tal que a desigualdade acima seja verdadeira.

Como, para cada $j=1, \ldots, n, i^{*}\left(d t_{j} \wedge d Z\right)$ é uma $(m+1)$-forma diferencial tem-se que existem funções

$$
f_{j} \in C^{\infty}\left(\bar{B}_{R} \times[0, t]\right)
$$

tais que

$$
i^{*}\left(d t_{j} \wedge d Z\right)(y)=f_{j}(y) d y_{1} \wedge \ldots \wedge d y_{m+1}, y \in \bar{B}_{R} \times[0, t] .
$$

Portanto $R_{\tau} u(x, t)$ é dado pela expressão

$$
(\tau / \pi)^{m / 2} \int_{\mathbb{R}^{m} \times[0, t]} \sum_{j=1}^{n}\left(e^{-\tau[Z(x, t)-Z(i(y))]^{2}}\right) L_{j}(h)(i(y)) u(i(y)) \operatorname{det} Z_{x}(i(y)) f_{j}(y) d y .
$$

Por fim, como para cada $j=1, \ldots, n$,

$$
L_{j}(h)(i(y)) u(i(y)) \operatorname{det} Z_{x}(i(y))
$$

são funções suaves com suporte contido em

$$
\bar{B}_{R} \times[0, t]
$$

existe constante $C_{0}>0$ tal que

$$
\int_{\mathbb{R}^{m} \times[0, t]}\left|L_{j}(h)(i(y)) u(i(y)) \operatorname{det} Z_{x}(i(y))\right| d y \leq C_{0},
$$

para quaiquer $j=1, \ldots, n$.

Concluimos, dessa forma, que

$$
\left|R_{\tau} u(x, t)\right| \leq C_{0}(\tau / \pi)^{m / 2} e^{\frac{-\tau R^{2}}{33}},(x, t) \in U,
$$

e fazendo $\tau \rightarrow+\infty$ obtemos que $\left|R_{\tau} u(x, t)\right| \rightarrow 0$ uniformemente em $U$.

Finalmente, mostremos que, para qualquer multi-índice $\alpha \in \mathbb{Z}^{N}, \partial^{\alpha} R_{\tau} u$ converge uniformemente para zero em $U$ quando $\tau \rightarrow+\infty$.

Afirmamos que, para qualquer multi-índice $\alpha \in \mathbb{Z}^{N}$, existem funções $g_{1}, \ldots, g_{|\alpha|}$ suaves definidas em $U \times \bar{B}_{R} \times[0, t]$ tais que

$$
\partial^{\alpha}\left(e^{-\tau\left[Z(x, t)-Z\left(x^{\prime}, t^{\prime}\right)\right]^{2}}\right)=\sum_{\eta=1}^{|\alpha|} \tau^{\eta} e^{-\tau\left[Z(x, t)-Z\left(x^{\prime}, t^{\prime}\right)\right]^{2}} g_{\eta}\left(x, t, x^{\prime}, t^{\prime}\right) .
$$


Assumindo a afirmação acima o resultado segue, visto que

$$
\partial^{\alpha} R_{\tau} u(x, t)=(\tau / \pi)^{m / 2} \int_{\mathbb{R}^{m} \times[0, t]} \sum_{\eta=1}^{|\alpha|} \sum_{j=1}^{n} \tau^{\eta} g_{\eta}(x, t, i(y)) L_{j}(h)(i(y)) u(i(y)) \operatorname{det} Z_{x}(i(y)) d y
$$

com

$$
g_{\eta}(x, t, i(y)) L_{j}(h)(i(y)) u(i(y)) \operatorname{det} Z_{x}(i(y))
$$

funções suaves com suporte contido em

$$
\bar{B}_{R} \times[0, t]
$$

para cada $(x, t) \in U$. Logo existe constante $C_{\alpha}>0$ tal que

$$
\int_{\mathbb{R}^{m} \times[0, t]} \sum_{\eta=1}^{|\alpha|} \sum_{j=1}^{n} \tau^{\eta}\left|g_{\eta}(x, t, i(y)) L_{j}(h)(i(y)) u(i(y)) \operatorname{det} Z_{x}(i(y))\right| d y \leq C_{\alpha},
$$

para quaiquer $j=1, \ldots, n,(x, t) \in \bar{U}$.

Dessa forma,

$$
\left|\partial^{\alpha} R_{\tau} u(x, t)\right| \leq C_{\alpha} \frac{\tau^{|\alpha|+m / 2}}{\pi^{m / 2}} e^{\frac{-\tau R^{2}}{33}},(x, t) \in U,
$$

$\tau \geq 1$ e concluimos que $\left|\partial^{\alpha} R_{\tau} u\right|$ converge uniformemente para zera em $U$ quando $\tau \rightarrow+\infty$ para qualquer para qualquer multi-índice $\alpha \in \mathbb{Z}^{N}$, ou seja,

$$
d_{C^{\infty}(U)}\left(R_{\tau} u, 0\right) \rightarrow 0
$$

quando $\tau \rightarrow+\infty$.

Provemos então que a afirmação é verdadeira. Faremos a prova por indução. Para $|\alpha|=0$ não há o que fazer. Suponha que a afirmação é válida para $|\alpha|=\alpha_{0}$ e provemos para $\partial_{k} \circ \partial^{\alpha}$.

Com efeito,

$$
\begin{aligned}
\partial_{k} \circ \partial^{\alpha}\left(e^{-\tau\left[Z(x, t)-Z\left(x^{\prime}, t^{\prime}\right)\right]^{2}}\right) & =\sum_{\eta=1}^{|\alpha|} \tau^{\eta} \partial_{k}\left(e^{-\tau\left[Z(x, t)-Z\left(x^{\prime}, t^{\prime}\right)\right]^{2}} g_{\eta}\left(x, t, x^{\prime}, t^{\prime}\right)\right)= \\
& =\sum_{\eta=1}^{|\alpha|} \tau^{\eta} \tau e^{-\tau\left[Z(x, t)-Z\left(x^{\prime}, t^{\prime}\right)\right]^{2}} \tilde{g}_{\eta}\left(x, t, x^{\prime}, t^{\prime}\right),
\end{aligned}
$$

em que

$$
\tilde{g}_{\eta}\left(x, t, x^{\prime}, t^{\prime}\right)=\partial_{k}\left(g_{\eta}\right)\left(x, t, x^{\prime}, t^{\prime}\right)+g_{\eta}\left(x, t, x^{\prime}, t^{\prime}\right) \sum_{l=1}^{m}(-2)\left(Z_{l}(x, t)-Z_{l}\left(x^{\prime}, t^{\prime}\right)\right) \partial_{k}\left(Z_{l}\right)(x, t)+
$$

provando a afirmação. 
Agora daremos uma ideia de como generalizar o resultado acima para o caso em que $\mathcal{L}$ é uma estrutura localmente integrável definida sobre $\Omega$ que um aberto de uma variedade suave difeomorfo a um aberto de $\mathbb{R}^{N}$ que contém a origem.

Note que podemos, partindo da estrutura localmente integrável $\mathcal{L}$ e do difeomorfismo $\xi$, definir uma estrutura $\hat{\mathcal{L}}$ sobre $\xi(\Omega)$ analogamente como foi feito na demonstração do Teorema de Frobenius.

Primeiramente considere a aplicação

$$
\begin{aligned}
\psi: \mathfrak{X}(\Omega) & \rightarrow \mathfrak{X}(\xi(\Omega)) \\
L & \mapsto \psi(L),
\end{aligned}
$$

em que

$$
\psi(L)(f)=L(f \circ \xi) \circ \xi^{-1} .
$$

Assim, como na demonstração do Teorema de Frobenius, sabendo que $\left\{L_{j p}\right\}_{j=1}^{n}$ é linearmente independente para cada $p \in \Omega$, concluimos que $\left\{\psi\left(L_{j}\right)_{q}\right\}_{j=1}^{n}$ é linearmente independente para cada $q \in \xi(\Omega)$.

Assim podemos definir a estrutura $\hat{\mathcal{L}}$ dada por

$$
\hat{\mathcal{L}}_{q}=\left[\psi\left(L_{1}\right)_{q}, \ldots, \psi\left(L_{n}\right)_{q}\right], q \in \xi(\Omega)
$$

Podemos transportar as funções $Z_{1}, \ldots, Z_{m}$ para $C^{\infty}(\xi(\Omega))$ da seguinte forma

$$
\hat{Z}_{k} \doteq Z_{j} \circ \xi^{-1}, k=1, \ldots, m .
$$

Dessa forma,

$$
d \hat{Z}_{k}\left(\psi\left(L_{j}\right)\right)=L_{j}\left(Z_{k} \circ \xi^{-1} \circ \xi\right) \circ \xi^{-1}=L_{j}\left(Z_{k}\right) \circ \xi^{-1}=0,
$$

para quaisquer $j=1, \ldots, n, k=1, \ldots, m$ e então $d \hat{Z}_{k q} \in \hat{\mathcal{L}}_{q}^{\perp}, q \in \xi(\Omega)$. Para concluirmos que a estrutura $\hat{\mathcal{L}}$ é localmente integrável, resta mostrarmos que $\left\{d \hat{Z}_{k q}\right\}_{k=1}^{m}$ é linearmente independente.

Sejam $\lambda_{1}, \ldots, \lambda_{m} \in \mathbb{C}$ e $q \in \xi(\Omega)$ tais que

$$
\sum_{k=1}^{m} \lambda_{k} d \hat{Z}_{k q}=0
$$

Além disso,

$$
d \hat{Z}_{k}\left(\psi\left(M_{l}\right)\right)=M_{l}\left(Z_{k}\right) \circ \xi^{-1}=\delta_{k l}
$$

$\log 0$

$$
0=\sum_{k=1}^{m} \lambda_{k} d \hat{Z}_{k q}\left(\psi\left(M_{l}\right)_{q}\right)=\lambda_{l}
$$

para $l=1, \ldots, m$. 
Então

$$
\hat{\mathcal{L}}_{q}^{\perp}=\left[d \hat{Z}_{1 q}, \ldots, d \hat{Z}_{m q}\right], q \in \xi(\Omega)
$$

e concluímos que $\hat{\mathcal{L}}$ é uma estrutura localmente integrável sobre $\xi(\Omega)$, o qual é um aberto de $\mathbb{R}^{N}$ que contém a origem e assim está nas condições do Teorema 7.1.2.

Agora basta utilizar o caso feito no início da seção para obtermos o resultado desejado.

\subsection{Versão Distribucional}

\subsubsection{Preliminares}

Antes de enunciarmos a versão distribucional do teorema, com o intuito de tornar mais clara sua demonstração, estabeleceremos alguns resultados auxiliares e também destacaremos algumas propriedades importantes dos objetos envolvidos em sua formulação.

Definição 7.2.1 (Espaço de Sobolev). Dado $k \in \mathbb{N}$ definimos os espaços de Sobolev como sendo

$$
H^{k}\left(\mathbb{R}^{N}\right) \doteq\left\{f \in L^{2}\left(\mathbb{R}^{N}\right) ; \partial^{\alpha} f \in L^{2}\left(\mathbb{R}^{N}\right), \forall \alpha \in \mathbb{Z}^{N} \operatorname{com}|\alpha| \leq k\right\}
$$

e $H^{-k}\left(\mathbb{R}^{N}\right)$ como sendo o espaço vetorial dual de $H^{k}\left(\mathbb{R}^{N}\right)$. Além disso, estes espaços são normados com normas dada por

$$
\|f\|_{k}=\sum_{|\alpha| \leq k}\left|\partial^{\alpha} f\right|_{L^{2}}
$$

se $f \in H^{k}\left(\mathbb{R}^{N}\right)$ e

$$
\|f\|_{-k}=\sup _{\phi \in H^{k}\left(\mathbb{R}^{N}\right),\|\phi\|_{k} \leq 1}|\langle f, \phi\rangle|
$$

se $f \in H^{-k}\left(\mathbb{R}^{N}\right)$.

A seguir apresentamos os conceitos de distribuição e conjunto frente de onda de uma distribuição.

Definição 7.2.2. Seja $\Omega \subset \mathbb{R}^{N}$. Uma distribuição u em $\Omega$ é uma aplicação linear

$$
u: C_{c}^{\infty}(\Omega) \rightarrow \mathbb{C}
$$

tal que para qualquer compacto $K \subset \Omega$ existem uma constante $C>0$ e um inteiro $k \in \mathbb{Z}$ de tal forma que vale a desigualdade

$$
|\langle u, \phi\rangle| \leq C \sum_{|\alpha| \leq k} \sup \left|\partial^{\alpha} \phi\right|,
$$

para quaisquer $\phi \in C_{c}^{\infty}(K)$. O conjunto de todas as distribuições em $\Omega$ é denotado por $\mathscr{D}^{\prime}(\Omega)$. 
Antes de definirmos conjunto frente de onda de uma distribuição precisamos da seguinte definição.

Definição 7.2.3. Sejam $u \in \mathscr{D}^{\prime}(\Omega)$ e $\left(x_{0}, \xi_{0}\right) \in \Omega \times(\mathbb{R}-\{0\})$. Dizemos que u é $C^{\infty}$ em $\left(x_{0}, \xi_{0}\right)$ se existirem $\phi \in C_{c}^{\infty}(\Omega)$, com $\phi \equiv 1$ numa vizinhança de $x_{0}$, e um cone aberto $\Gamma \subset \mathbb{R}^{N}-\{0\}$ contendo $\xi_{0}$ tais que

$$
|\mathcal{F}(\phi u)| \leq C_{k}(1+|\xi|)^{-k}, \xi \in \Gamma
$$

em que $\mathcal{F}$ denota a transformada de Fourier, $C_{k}>0$ é uma constante e $k \in \mathbb{N}$.

Definição 7.2.4 (Conjunto frente de onda). Seja $u \in \mathscr{D}^{\prime}(\Omega)$. O conjunto frente de onda de u é a coleção dos pontos $(x, \xi) \in \Omega \times\left(\mathbb{R}^{N}-\{0\}\right)$ tais que u não é $C^{\infty}$ em $(x, \xi)$. O conjunto frente de onda da distribuição u é denotado por $W F(u)$.

A principal referência destas definições é [2].

Observação 7.2.5. Sejam $u \in \mathscr{D}^{\prime}(W)$ e $L \in \mathfrak{X}(W)$. Por um lado, se

$$
\left(x_{1}, \ldots, x_{N}\right)
$$

for um sistema de coordenadas locais, o campo pode ser descrito da seguinte forma

$$
L=\sum_{j=1}^{N} a_{j} \frac{\partial}{\partial x_{j}}, a_{j}=L\left(x_{j}\right)
$$

Por outro, de [2] sabemos que

$$
\left\langle\partial^{\alpha} u, \phi\right\rangle \doteq(-1)^{|\alpha|}\left\langle u, \partial^{\alpha} \phi\right\rangle, \phi \in C_{c}^{\infty}(W), \alpha \in \mathbb{Z}^{N}
$$

e assim

$$
\left\langle\frac{\partial u}{\partial x_{j}}, \phi\right\rangle=(-1)\left\langle u, \frac{\partial \phi}{\partial x_{j}}\right\rangle, \phi \in C_{c}^{\infty}(W), j=1, \ldots, N
$$

Dessa forma,

$$
\langle L u, \phi\rangle=\left\langle\sum_{j=1}^{N} a_{j} \frac{\partial u}{\partial x_{j}}, \phi\right\rangle=-\left\langle u, \sum_{j=1}^{N} \frac{\partial\left(a_{j} \phi\right)}{\partial x_{j}}\right\rangle, \phi \in C_{c}^{\infty}(W) .
$$

Seja $u \in \mathscr{D}^{\prime}(W)$ tal que $\mathcal{L} u=0$, isto é,

$$
L_{j} u=0, j=1, \ldots, n .
$$

Faremos uso do seguinte resultado de [2]: 
Teorema 7.2.6. Sejam $u \in \mathscr{D}^{\prime}(\Omega)$ e $P(x, D)$ um operador diferencial parcial linear com coeficientes em $\Omega$, onde $\Omega \subset \mathbb{R}^{N}$ é um aberto. Então

$$
W F(u) \subset W F(P u) \cup V_{P},
$$

em que

$$
V_{P}=\left\{(x, \xi) \in \Omega \times\left(\mathbb{R}^{N}-\{0\}\right) ; P_{m}(x, \xi)=0\right\}
$$

é o conjunto característico de $P(x, D), m$ sua ordem, $P_{m}(x, \xi)=\sum_{|\alpha|=m} a_{\alpha}(x) \xi^{\alpha}$ é seu símbolo principal e $W F(u)$ indica o conjunto frente de onda da distribuição $u$.

No nosso caso os campos $L_{1}, \ldots, L_{n}$ podem ser vistos como operadores diferenciais parciais lineares de ordem 1 . Note que $\mathcal{L}_{p}=\left[L_{1 p}, \ldots, L_{n p}\right], p \in W$ e $((x, t), \xi) \in T^{0}$ se, e somente se,

$$
\sum_{k=1}^{m} a_{j k}(x, t) \xi_{k}+\sum_{l=1}^{n} b_{j l}(x, t) \xi_{l+m}=0, j=1, \ldots, n,
$$

ou seja, se, e somente se, $((x, t), \xi) \in T_{j}^{0}, j=1, \ldots, n$ onde $T_{j}^{0}$ é o conjunto característico de $\mathcal{L}_{j p}=\left[L_{j p}\right], p \in W$.

Além disso, como

$$
\mathcal{L}_{p}=\mathcal{L}_{p 1} \oplus \ldots \oplus \mathcal{L}_{p n}, p \in W
$$

então

$$
\mathcal{L}_{p}^{\perp}=\cap_{j=1}^{p} \mathcal{L}_{p j}^{\perp}, p \in W
$$

Logo

$$
T_{p}^{0}=\mathcal{L}_{p}^{\perp} \cap T_{p}^{*} \mathbb{R}^{N}=\cap_{j=1}^{n} \mathcal{L}_{p j}^{\perp} \cap T_{p}^{*} \mathbb{R}^{N}=\cap_{j=1}^{n} T_{p j}^{0},
$$

ou seja,

$$
T^{0}=\cap_{j=1}^{n} T_{j}^{0}
$$

Sabemos também que

$$
T_{j}^{0}=V_{L_{j}} \cup\left(\mathbb{R}^{N} \times\{0\}\right),
$$

juntando os dois fatos obtemos

$$
T^{0}=\cap_{j=1}^{n} V_{L_{j}} \cup\left(\mathbb{R}^{N} \times\{0\}\right) .
$$

Para cada $j=1, \ldots, n$, de 7.2 .6 e sendo $L_{j} u=0$ temos

$$
W F(u) \subset W F\left(L_{j} u\right) \cup V_{L_{j}}=V_{L_{j}},
$$

então

$$
W F(u) \subset \cap_{j=1}^{n} V_{L_{j}} \subset T^{0} .
$$


Como $\bar{V} \subset W, \bar{V}$ é compacto e $W$ aberto, é possível obter $0<R^{\prime}<R^{\prime \prime}$ tal que

$$
\bar{V} \subset V^{\prime} \doteq\left\{(x, t) ;|x|,|t|<R^{\prime}\right\} \subset \overline{V^{\prime}} \subset V^{\prime \prime} \doteq\left\{(x, t) ;|x|,|t|<R^{\prime \prime}\right\} \subset W .
$$

Nessas condições, afirmamos que

$$
W F(u) \cap\left\{(x, t, 0, \lambda) \in V^{\prime \prime} \times \mathbb{R}^{m} \times \mathbb{R}^{n} ; \lambda \neq 0\right\}=\emptyset .
$$

Com efeito, suponha que existam $(x, t) \in V^{\prime \prime}$ e $\xi \in \mathbb{R}^{n}-\{0\}$ tais que $(x, t, 0, \xi) \in$ $T^{0}=\cap_{j=1}^{n} T_{j}^{0}$. Assim, como

$$
L_{j}=\frac{\partial}{\partial t_{j}}+\sum_{k=1}^{m} \lambda_{j k} \frac{\partial}{\partial x_{k}}, j=1, \ldots, n,
$$

tem-se que

$$
\xi_{j}=\xi_{j}+\sum_{k=1}^{m} \lambda_{j k}(x, t) 0=0, j=1, \ldots, n
$$

que é uma contradição, pois assumimos $\xi=\left(\xi_{1}, \ldots, \xi_{n}\right) \neq 0$.

Portanto

$$
\left\{(x, t, 0, \lambda) \in V^{\prime \prime} \times \mathbb{R}^{m} \times \mathbb{R}^{n} ; \lambda \neq 0\right\} \subset\left(T^{0}\right)^{c} \subset(W F(u))^{c} .
$$

Dessa maneira, o conjunto $W F(h u)$ está contido em $T^{0}$, então não intercepta

$$
\left\{(x, t, 0, \lambda) \in V^{\prime \prime} \times \mathbb{R}^{m} \times \mathbb{R}^{n} ; \lambda \neq 0\right\} .
$$

e de [5] obtemos que

$$
u \in C^{\infty}\left(\left\{|t| \leq R^{\prime}\right\} ; \mathscr{D}^{\prime}\left(\left\{|x|<R^{\prime}\right\}\right)\right) .
$$

Além do mais, se $\chi \in C^{\infty}\left(\mathbb{R}^{m}\right)$ com $\chi$ identicamente 1 numa vizinhança de

$$
\left\{|x| \leq R^{\prime}\right\}
$$

e suporte contido em

$$
\left\{|x|<R^{\prime \prime}\right\}
$$

consequentemente tem-se que

$$
\hat{u} \doteq \chi u \in C^{\infty}\left(\left\{|t| \leq R^{\prime}\right\} ; \mathscr{E}^{\prime}\left(\left\{|x|<R^{\prime}\right\}\right)\right) \subset C^{0}\left(\left\{|t| \leq R^{\prime}\right\} ; \mathscr{E}^{\prime}\left(\left\{|x|<R^{\prime}\right\}\right)\right) .
$$

Por simplicidade e como nosso resultado principal vale apenas localmente, denotaremos $\hat{u}$ simplismente por $u$. Observe que $\hat{u}(\cdot, t)=u(\cdot, t)$ em numa vizinhança de $\left\{|x| \leq R^{\prime}\right\}$ para qualquer $t$.

Como $\left\{|t| \leq R^{\prime}\right\}$ é compacto e pela continuidade na variável $t$, estando $\mathscr{D}^{\prime}(\{|x|<$ $\left.\left.R^{\prime}\right\}\right)$ munido da topologia fraca estrela, obtemos que $u(\cdot, t)$ é uniformemente contínua na variável $t$, isto é, dado $\varepsilon>0$, para cada $\phi \in C^{\infty}\left(\left\{|x|<R^{\prime}\right\}\right)$, existe $\delta=\delta(\phi)>0$ tal que

$$
|\langle u(\cdot, t)-u(\cdot, s), \phi\rangle|<\varepsilon, \forall|t-s|<\delta,|t|,|s| \leq R^{\prime}
$$


Por outro lado, como, para cada $t, u(\cdot, t)$ é uma distribuição com suporte compacto, então também é uma distribuição de ordem finita, ou seja, existe $N_{t} \in \mathbb{N}$ tal que para cada compacto $K \subset\left\{|x|<R^{\prime}\right\}$, podemos encontrar constante $C=C(K)>0$ tais que

$$
|\langle u(\cdot, t), \phi\rangle| \leq C \sum_{|\alpha| \leq N_{t}} \sup _{K}\left|\partial^{\alpha} \phi\right|, \phi \in C_{c}^{\infty}(K) .
$$

Logo, dados $\varepsilon>0, K \subset\left\{|x|<R^{\prime}\right\}$ e $\phi \in C_{c}^{\infty}(K)$ existe $\delta=\delta(\phi)>0$ tal que vale 7.2. Como $\left\{|t| \leq R^{\prime}\right\}$ é compacto, existem $t_{1}, \ldots, t_{l}$ contidos em neste compacto tais que

$$
\left\{|t| \leq R^{\prime}\right\} \subset \cup_{j=1}^{l} B\left(t_{j} ; \delta\right)
$$

Além disso, para $j=1, \ldots, l$, existem $C_{j}>0$ uma constante e $N_{j} \in \mathbb{N}$ tais que vale 7.3.

Tome

$$
C \doteq \max _{1 \leq j \leq l} C_{j}, N_{0} \doteq \max _{1 \leq j \leq l} N_{j}
$$

Assim, para $|t| \leq R^{\prime}$ existe $1 \leq j \leq l$, tal que $\left|t-t_{j}\right|<\delta$ e assim

$$
|\langle u(\cdot, t), \phi\rangle|<\left|\left\langle u\left(\cdot, t_{j}\right), \phi\right\rangle\right|+\varepsilon \leq C \sum_{|\alpha| \leq N_{0}} \sup _{K}\left|\partial^{\alpha} \phi\right|+\varepsilon .
$$

Visto que a desigualdade acima vale para qualquer $\varepsilon>0$, podemos calcular o limite quando $\varepsilon \rightarrow 0$ e obtemos

$$
|\langle u(\cdot, t), \phi\rangle| \leq C \sum_{|\alpha| \leq N_{0}} \sup _{K}\left|\partial^{\alpha} \phi\right|
$$

isto é, concluímos que $u(\cdot, t) \in \mathscr{D}_{N_{0}}^{\prime}\left(\left\{|x|<R^{\prime \prime}\right\}\right)$.

Por fim, tem-se que

$$
u(\cdot, t) \in H^{k_{0}}\left(\left\{|x|<R^{\prime \prime}\right\}\right),
$$

para algum $k_{0} \in \mathbb{Z}$, com $k_{0}+\frac{N_{0}}{2}<\frac{-m}{2}$, qualquer que seja $|t| \leq R^{\prime}$. Em outras palavras

$$
u \in C^{0}\left(\left\{|t| \leq R^{\prime}\right\} ; H^{k_{0}}\left(\left\{|x|<R^{\prime \prime}\right\}\right)\right)
$$

Agora faremos alguns lemas que nos fornecem subespaços densos de funções mais regulares em

$$
X_{k} \doteq\left\{v \in C^{0}\left(\left\{|t| \leq R^{\prime}\right\} ; H^{k}\left(\mathbb{R}^{m}\right)\right) ; \operatorname{supp}(v(\cdot, t)) \subset B_{R^{\prime \prime}}(0)\right\}, k \in \mathbb{Z}
$$

Esta densidade é essencial para a demonstração da fórmula de Baouendi-Treves e para obtermos essa densidade lançamos mão de resultados sofisticados como o Teorema de Arzela-Ascoli. 
Observação 7.2.7. É importante ressaltar que os espaços

$$
Y_{k} \doteq C^{0}\left(\left\{|t| \leq R^{\prime}\right\} ; H^{k}\left(\left\{|x|<R^{\prime \prime}\right\}\right)\right), k \in \mathbb{Z}
$$

são normados, a saber

$$
\|u\|_{Y_{k}} \doteq \sup _{|t| \leq R^{\prime}}\|u(\cdot, t)\|_{k}, u \in Y_{k},
$$

sendo que $\|\cdot\|_{k}$ denota a norma do espaço de Sobolev $H^{k}$.

Lema 7.2.8. Para cada $k \in \mathbb{N}, r_{1}, r_{2}>0$, considere o seguinte espaço

$$
X \doteq\left\{f \in C_{c}^{0}\left(\left\{|t| \leq r_{2}\right\} ; H^{k}\left(\mathbb{R}^{m}\right)\right) ; \operatorname{supp}(f(\cdot, t)) \subset B_{r_{1}}(0), \forall|t| \leq r_{2}\right\}
$$

Então dada $v \in X$ existe uma sequência $\left\{\phi_{l}\right\}_{l \in \mathbb{N}}$ em $C^{\infty}\left(\bar{B}_{r_{1}+1} \times \bar{B}_{r_{2}+1}\right)$ que converge para $v$ na norma de $X$.

Demonstração. A convergência é dividiva em duas partes. Sejam $v \in X$ e $\left(\rho_{l}\right)_{l \in \mathbb{N}} \subset$ $C_{c}^{\infty}\left(\mathbb{R}^{m}\right)$ uma sequência regularizante. Para cada $t \in \mathbb{R}^{n}$, considere $\rho_{l} * v(\cdot, t)$, isto é,

$$
\rho_{l} * v(x, t)=\int_{\mathbb{R}^{m}} \rho(y) v(x-y, t) d y .
$$

Temos

$$
\operatorname{supp}\left(\rho_{l} * v(\cdot, t)\right) \subset \operatorname{supp}\left(\rho_{l}\right)+\operatorname{supp}(v(\cdot, t)) \subset \bar{B}_{r_{1}+1},
$$

e como $\rho_{l} * v(\cdot, t)$ é suave temos que

$$
\rho_{l} * v \in C_{c}^{0}\left(\left\{|t| \leq r_{2}\right\} ; C_{c}^{\infty}\left(\bar{B}_{r_{1}+1}\right)\right) .
$$

Como $v \in X$, sabemos que $\partial_{x}^{\alpha} v(\cdot, t) \in L^{2}\left(\mathbb{R}^{m}\right), 0 \leq|\alpha| \leq k$ e $t \in \mathbb{R}^{n}$. Além do mais, para cada $t$ fixo, quando $l \rightarrow \infty$ vale que

$$
\left\|\partial^{\alpha}\left(\rho_{l} * v(\cdot, t)\right)-v(\cdot, t)\right\|_{L^{2}\left(\mathbb{R}^{m}\right)}=\left\|\rho_{l} * \partial^{\alpha} v(\cdot, t)-v(\cdot, t)\right\|_{L^{2}\left(\mathbb{R}^{m}\right)} \rightarrow 0,
$$

ou seja,

$$
\left\|\rho_{l} * v(\cdot, t)-v(\cdot, t)\right\|_{k} \rightarrow 0
$$

Defina

$$
f_{l}(t) \doteq\left\|\rho_{l} * v(\cdot, t)-v(\cdot, t)\right\|_{k}, t \in K \doteq \bar{B}_{r_{2}}, l \in \mathbb{N} .
$$

Note que $f_{l} \in C^{0}(K ; \mathbb{R})$ e que por 7.4 tal sequência converge pontualmente para 0 quando $l \rightarrow \infty$.

Vamos mostrar que esta sequência de funções satisfaz as hipóteses do teorema de Arzela-Ascoli. Para tanto, observe que pela desigualdade de Young

$$
\left\|\rho_{l} * \partial^{\alpha} v(\cdot, t)\right\|_{L^{2}\left(\mathbb{R}^{m}\right)} \leq\left\|\rho_{l}\right\|_{L^{1}\left(\mathbb{R}^{m}\right)}\left\|\partial^{\alpha} v(\cdot, t)\right\|_{L^{2}\left(\mathbb{R}^{m}\right)}=\left\|\partial^{\alpha} v(\cdot, t)\right\|_{L^{2}\left(\mathbb{R}^{m}\right)}
$$


então

$$
\left\|\rho_{l} * v(\cdot, t)\right\|_{L^{2}\left(\mathbb{R}^{m}\right)} \leq\|v(\cdot, t)\|_{k} .
$$

Além disso, como $v(\cdot, t)$ é contínua na variável $t$ existe constante $C>0$ tal que

$$
\|v(\cdot, t)\|_{k} \leq C, \forall t \in K
$$

Logo

$$
\left|f_{l}(t)\right| \leq 2\|v(\cdot, t)\|_{k} \leq 2 C, \forall t \in K, l \in \mathbb{N}
$$

isto é, a sequência é uniformemente limitada.

Novamente por ser $v(\cdot, t)$ é contínua na variável $t$ e $K$ compacto, $v(\cdot, t)$ é uniformemente contínua.

Assim dado $\varepsilon>0$ existe $\delta>0$ tal que

$$
\|v(\cdot, t)-v(\cdot, s)\|_{k}<\frac{\varepsilon}{2} \text {, toda vez que } t, s \in K \text { cumprem }|t-s|<\delta .
$$

Então

$$
\left|f_{l}(t)-f_{l}(s)\right|=\left\|\rho_{l} *(v(\cdot, t)-v(\cdot, s))-(v(\cdot, t)-v(\cdot, s))\right\|_{k} \leq 2\|(v(\cdot, t)-v(\cdot, s))\|_{k}<\varepsilon
$$

para quaisquer $t, s \in K \operatorname{com}|t-s|<\delta$ e $l \in \mathbb{N}$ e dessa forma $f_{l}$ é equicontínua.

Agora podemos aplicar o teorema de Arzela-Ascoli para concluir a existência de uma subsequência $\left(f_{l_{j}}\right)_{j \in \mathbb{N}}$ que converge uniformemente para 0 , isto é,

$$
\sup _{K}\left\|\rho_{l_{j}} * v(\cdot, t)-v(\cdot, t)\right\|_{k}=\sup _{K} f_{l_{j}}(t) \rightarrow 0
$$

quando $j \rightarrow \infty$. Por simplicidade, denotamos tal subsequência por $\rho_{l} * v$.

Agora tome $\psi_{l} \in C_{c}^{\infty}\left(\mathbb{R}^{n}\right)$ sequência regularizante e considere $\psi_{l} * \rho_{l} * v$. Destacamos que é possível realizar este produto de convolução, uma vez que

$$
\partial^{\alpha}\left(\rho_{l} * v\right)=\rho_{l} * \partial^{\alpha} v \in C_{c}^{0}\left(\bar{B}_{r_{2}} ; C_{c}^{0}\left(\bar{B}_{r_{1}+1}\right)\right) \equiv C_{c}^{0}\left(\bar{B}_{r_{1}+1} \times \bar{B}_{r_{2}}\right)
$$

para $0 \leq|\alpha| \leq k$. Assim

$$
\psi_{l} *\left(\rho_{l} * v\right)(x, t)=\int_{\mathbb{R}^{n}} \psi_{l}(s) \rho_{l} * v(x, t-s) d s,
$$

analogamente ao caso anterior, para $x \in \mathbb{R}^{m}$,

$$
\psi_{l} *\left(\rho_{l} * v\right)(x, \cdot) \in C_{c}^{\infty}\left(\bar{B}_{r_{2}+1}\right),
$$

então

$$
\psi_{l} *\left(\rho_{l} * v\right) \in C_{c}^{\infty}\left(\bar{B}_{r_{1}+1} \times \bar{B}_{r_{2}+1}\right)
$$


Temos também que

$$
\begin{aligned}
\left|\rho_{l} * \partial^{\alpha} v(x, t-s)-\rho_{l} * \partial^{\alpha} v(x, t)\right| & \leq \int_{\mathbb{R}^{m}} \rho_{l}(x-y)\left|\partial^{\alpha} v(y, t-s)-\partial^{\alpha} v(y, t)\right| d y \leq \\
& \leq\left\|\partial^{\alpha} v(\cdot, t-s)-\partial^{\alpha} v(\cdot, t)\right\|_{L^{2}\left(\mathbb{R}^{m}\right)} \leq \\
& \leq\|v(\cdot, t-s)-v(\cdot, t)\|_{k} .
\end{aligned}
$$

Mais uma vez, utilizando a continuidade uniforme na variável $t$ de $v$ no compacto $K$, dado $\varepsilon>0$ tome $l_{0} \in \mathbb{N}$ tal que $1 / l_{0}<\delta$ portanto

$$
\begin{array}{r}
\left|\psi_{l} *\left(\rho_{l} * \partial^{\alpha} v\right)(x, t)-\left(\rho_{l} * \partial^{\alpha} v\right)(x, t)\right| \leq \\
\leq \int_{B_{\frac{1}{l}}} \psi_{l}(s) \mid \rho_{l} * \partial^{\alpha} v(x, t-s)-\left(\rho_{l} * \partial^{\alpha} v(x, t) \mid d s \leq\right. \\
\leq\|v(\cdot, t-s)-v(\cdot, t)\|_{k}<\varepsilon .
\end{array}
$$

Resumidamente, obtivemos que

$$
\left|\psi_{l} *\left(\rho_{l} * \partial^{\alpha} v\right)(x, t)-\left(\rho_{l} * \partial^{\alpha} v\right)(x, t)\right|<\varepsilon, \forall t \in K, x \in \mathbb{R}^{m}, l \geq l_{0} .
$$

Logo

$$
\begin{array}{r}
\int_{\mathbb{R}^{m}}\left|\psi_{l} *\left(\rho_{l} * \partial^{\alpha} v\right)(x, t)-\left(\rho_{l} * \partial^{\alpha} v\right)(x, t)\right|^{2} d x \leq \\
\leq \int_{\bar{B}_{r_{1}+1}}\left|\psi_{l} *\left(\rho_{l} * \partial^{\alpha} v\right)(x, t)-\left(\rho_{l} * \partial^{\alpha} v\right)(x, t)\right|^{2} d x< \\
<\varepsilon^{2} \mu\left(\bar{B}_{r_{1}+1}\right), \forall t \in K, l \geq l_{0},
\end{array}
$$

ou seja,

$$
\sup _{K}\left\|\psi_{l} *\left(\rho_{l} * v\right)(\cdot, t)-\left(\rho_{l} * v\right)(\cdot, t)\right\|_{k}<\varepsilon \sqrt{\mu\left(\bar{B}_{r_{1}+1}\right)} .
$$

Como $\varepsilon>0$ é arbitrário concluímos a demonstração.

Lema 7.2.9. Para cada $k \in \mathbb{N}, r_{1}, r_{2}>0$, considere o seguinte espaço

$$
X \doteq\left\{f \in C_{c}^{0}\left(\left\{|t| \leq r_{2}\right\} ; H^{-k}\left(\mathbb{R}^{m}\right)\right) ; \operatorname{supp}(f(\cdot, t)) \subset B_{r_{1}}(0), \forall|t| \leq r_{2}\right\}
$$

Então dada $v \in X$ existe uma sequência $\left\{\phi_{l}\right\}_{l \in \mathbb{N}}$ em $C^{\infty}\left(\bar{B}_{r_{1}+1} \times \bar{B}_{r_{2}+1}\right)$ que converge para $v$ na norma de $X$.

Demonstração. A convergência é dividiva em duas partes. Sejam $v \in X$ e $\left(\rho_{l}\right)_{l \in \mathbb{N}} \subset$ $C_{c}^{\infty}\left(\mathbb{R}^{m}\right)$ sequência regularizante. Para cada $t \in \mathbb{R}^{n}$, considere $v(\cdot, t) * \rho_{l}$, isto é,

$$
\left\langle v(\cdot, t) * \rho_{l}, f\right\rangle=\left\langle v(\cdot, t),\left(\rho_{l} * f^{\vee}\right)^{\vee}\right\rangle, f \in H^{k}\left(\mathbb{R}^{m}\right) .
$$


Observação 7.2.10. Se $u \in H^{-k}\left(\mathbb{R}^{m}\right)$ com suporte contido em $\bar{B}_{r_{1}}$ então

$$
\|u\|_{-k}=\sup _{f \in H^{k},\|f\|_{k} \leq 1}|\langle u, \phi f\rangle|
$$

onde $\phi \in C_{c}^{\infty}\left(\mathbb{R}^{m}\right)$ que vale 1 numa vizinhança de $\bar{B}_{r_{1}}$ e tem suporte contido em $\bar{B}_{r_{1}+1}$.

Com efeito,

$$
f=\phi f+(1-\phi) f
$$

temos que $(1-\phi) f=0$ numa vizinhança de $\bar{B}_{r_{1}}$ e então

$$
\operatorname{supp}((1-\phi) f) \cap \bar{B}_{r_{1}}=\emptyset
$$

Logo

$$
\langle u, f\rangle=\langle u, \phi f\rangle, \forall f \in H^{k}
$$

Além do mais, se $C=\max _{0 \leq|\alpha| \leq k} \sup \left|\partial^{\alpha} \phi\right|$ então

$$
\partial^{\alpha}(\phi f)=\phi \partial^{\alpha} f+\sum_{0 \leq|\beta|<|\alpha|}\left(\partial^{\beta} \phi\right)\left(\partial^{\alpha-\beta} f\right)
$$

e

$\left\|\partial^{\alpha}(\phi f)\right\|_{L^{2}} \leq\left\|\phi \partial^{\alpha} f\right\|_{L^{2}}+\sum_{0 \leq|\beta|<|\alpha|}\left\|\partial^{\beta} \phi \partial^{\alpha-\beta} f\right\|_{L^{2}} \leq C\left(\|f\|_{k}+\sum_{0 \leq|\beta|<|\alpha|}\|f\|_{k}\right) \leq \tilde{C}\|f\|_{k}$ ou seja, existe constante $\tilde{C}$ tal que $\|\phi f\|_{k} \leq \tilde{C}$, para $f \in H^{k}$ com $\|f\|_{k} \leq 1$.

Por fim, se $f \in H^{k}\left(\mathbb{R}^{m}\right)$ com suporte contido em $B_{r_{1}+1}$ para $0 \leq|\alpha| \leq k$ temos

$$
\partial^{\alpha}\left(\rho_{l} * f\right)(x)=\int_{\mathbb{R}^{m}} \rho_{l}(x-y) \partial^{\alpha} f(y) d y, x \in B_{r_{1}+1},
$$

pois $\operatorname{supp}\left(\rho_{l} * f\right) \subset B_{r_{1}+1}$, então

$$
\begin{gathered}
\left|\partial^{\alpha}\left(\rho_{l} * f\right)(x)\right| \leq\left\|\partial^{\alpha} f\right\|_{L^{2}}\left\|\rho_{l}(x-\cdot)\right\|=\left\|\partial^{\alpha} f\right\|_{L^{2}} \leq\|f\|_{k}, x \in B_{r_{1}+1} \\
\left\|\partial^{\alpha}\left(\rho_{l} * f\right)\right\|_{L^{2}} \leq\|f\|_{k} \sqrt{\mu\left(B_{r_{1}+1}\right)}
\end{gathered}
$$

e assim concluimos que

$$
\left\|\rho_{l} * f\right\|_{k} \leq\|f\|_{k} \sqrt{\mu\left(B_{r_{1}+1}\right)}
$$

Temos

$$
\operatorname{supp}\left(v(\cdot, t) * \rho_{l}\right) \subset \operatorname{supp}\left(\rho_{l}\right)+\operatorname{supp}(v(\cdot, t)) \subset \bar{B}_{r_{1}+1},
$$

$v(\cdot, t) * \rho_{l}$ é suave e então

$$
v * \rho_{l} \in C_{c}^{0}\left(\left\{|t| \leq r_{2}\right\} ; C_{c}^{\infty}\left(\bar{B}_{r_{1}+1}\right)\right) .
$$


Dessa forma, para $f \in H^{k}\left(\mathbb{R}^{m}\right)$ com $\|f\|_{k} \leq 1$ vale

$$
\begin{array}{r}
\left|\left\langle v(\cdot, t) * \rho_{l}-v(\cdot, t), \phi f\right\rangle\right|=\left|\left\langle v(\cdot, t),\left(\rho_{l} *(\phi f)^{\vee}\right)^{\vee}-\phi f\right\rangle\right| \leq \\
\leq\|v(\cdot, t)\|_{-k}\left\|\left(\rho_{l} *(\phi f)^{\vee}\right)^{\vee}-\phi f\right\|_{k}=\|v(\cdot, t)\|_{-k}\left\|\rho_{l} *(\phi f)^{\vee}-(\phi f)^{\vee}\right\|_{k} \leq \\
\left.\leq\|v(\cdot, t)\|_{-k}\left(\left\|\rho_{l} *(\phi f)^{\vee}\right\|_{k}+\| \phi f\right)^{\vee} \|_{k}\right) \leq \tilde{C}\|v(\cdot, t)\|\left\|_{-k}\right\| f^{\vee} \|_{k}= \\
=\tilde{C}\|v(\cdot, t)\|_{-k}\|f\|_{k} \leq \tilde{C}\|v(\cdot, t)\|_{-k} .
\end{array}
$$

Como $\overline{C_{c}^{\infty}\left(\mathbb{R}^{m}\right)}=H^{-k}\left(\mathbb{R}^{m}\right)$, temos que para cada $t \in K=\bar{B}_{r_{2}}$ vale

$$
\left\|v(\cdot, t) * \rho_{l}-v(\cdot, t)\right\|_{-k} \rightarrow 0
$$

quando $l \rightarrow \infty$. Sendo assim, tome

$$
g_{l}(t) \doteq\left\|v(\cdot, t) * \rho_{l}-v(\cdot, t)\right\|_{-k} \in C^{0}(K ; \mathbb{R}) .
$$

Note que, como vimos, $g_{l}$ converge pontualmente para 0. Como $v$ é contínua na variável $t$ e $K$ compacto existe constante $M>0$ tal que

$$
\|v(\cdot, t)\|_{-k} \leq M, t \in K
$$

e então

$$
\left|g_{l}(t)\right| \leq \tilde{C} M, t \in K, l \in \mathbb{N}
$$

Agora mostremos que a sequência de funções é equicontínua. Com efeito, para $t, s \in K$ tem-se que

$$
\left|g_{l}(t)-g_{l}(s)\right| \leq\left\|(v(\cdot, t)-v(\cdot, s)) * \rho_{l}\right\|_{-k}+\|v(\cdot, t)-v(\cdot, s)\|_{-k} .
$$

Por outro lado,

$$
\begin{array}{r}
\left|\left\langle(v(\cdot, t)-v(\cdot, s)) * \rho_{l}, \phi f\right\rangle\right|=\left|\left\langle v(\cdot, t)-v(\cdot, s),\left(\rho *(\phi f)^{\vee}\right)^{\vee}\right\rangle\right| \leq \\
\leq\|v(\cdot, t)-v(\cdot, s)\|_{-k}\left\|\left(\rho *(\phi f)^{\vee}\right)^{\vee}\right\|_{k}=\|v(\cdot, t)-v(\cdot, s)\|_{-k}\left\|\left(\rho *(\phi f)^{\vee}\right)\right\|_{k} \leq \\
\leq\|v(\cdot, t)-v(\cdot, s)\|_{-k} C_{1}\left\|(\phi f)^{\vee}\right\|_{k} \leq C_{2}\|v(\cdot, t)-v(\cdot, s)\|_{-k},
\end{array}
$$

onde $C_{1}=\sqrt{\mu\left(B_{r_{1}+1}\right)}$ e $C_{2}=C_{1} \tilde{C}$. Mais uma vez, utilizando que $v$ é contínua no compacto $K$ e portanto uniformemente contínua obtemos que a sequência é equicontínua. Pelo Teorema de Arzela-Ascoli, existe subsequência $\left(g_{l_{j}}\right)_{j \in \mathbb{N}}$ que converge uniformemente para 0, ou seja,

$$
\sup _{t \in K}\left\|v(\cdot, t) * \rho_{l_{j}}-v(\cdot, t)\right\|_{-k} \rightarrow 0
$$

quando $j \rightarrow \infty$.

Antes de provarmos a segunda parte da convergência, ressaltamos que para $f \in H^{k}$ com norma menor ou igual a 1 vale

$$
\left|\left\langle v(\cdot, t) * \rho_{l}, f\right\rangle\right|=\mid\left\langle v(\cdot, t),\left(\rho *(f)^{\vee}\right)^{\vee}\right| \leq C_{1}\|v(\cdot, t)\|_{-k} .
$$


Agora tome $\psi_{l} \in C_{c}^{\infty}\left(\mathbb{R}^{n}\right)$ sequência regularizante e considere $\psi_{l} * \rho_{l} * v$. Dado $\varepsilon>0$, existe $\delta>0$ tal que

$$
\|v(\cdot, t)-v(\cdot, s)\|_{-k}<\varepsilon, \forall|t-s|<\delta .
$$

Considere $l_{0}>1 / \delta$, assim para $f \in H^{k}\left(\mathbb{R}^{m}\right)$ com norma menor ou igual a 1 temos

$$
\left\langle\psi_{l} *\left(\rho_{l} * v\right)(\cdot, t)-\left(\rho_{l} * v\right)(\cdot, t), f\right\rangle=\int_{B_{\frac{1}{l}}} \psi_{l}(s)\left\langle\rho_{l} * v(\cdot, t-s)-\rho_{l} * v(\cdot, t), f\right\rangle d s
$$

$\log O$

$$
\begin{aligned}
\left|\left\langle\psi_{l} *\left(\rho_{l} * v\right)(\cdot, t)-\left(\rho_{l} * v\right)(\cdot, t), f\right\rangle\right| & \leq C_{1} \int_{B_{\frac{1}{l}}} \psi_{l}(s)\|v(\cdot, t-s)-v(\cdot, t)\|_{-k} d s< \\
& <C_{1} \varepsilon \int_{B_{\frac{1}{l}}} \psi_{l}(s) d s=C_{1} \varepsilon
\end{aligned}
$$

para $l \geq l_{0}$, isto é,

$$
\left\|\psi_{l} *\left(\rho_{l} * v\right)(\cdot, t)-\left(\rho_{l} * v\right)(\cdot, t)\right\|_{-k}<C_{1} \varepsilon
$$

como $\varepsilon>0$ é arbitrário, fazendo $\varepsilon \rightarrow 0$ concluimos a demonstração do lema.

Na prova da versão para distribuições também fazemos uso do operador $G_{\tau}$, porém ainda temos que definir este operador para distribuições.

Como, para cada $|t| \leq R^{\prime}, u(\cdot, t) \in H^{k}\left(\mathbb{R}^{m}\right)$ com suporte contido em $B_{R^{\prime \prime}}(0)$ definimos

$$
G_{\tau} u(x, t) \doteq\left\langle u(\cdot, t),(\tau / \pi)^{m / 2} e_{\tau}(Z(x, t), \cdot, t) h(\cdot) \operatorname{det} Z_{x}(\cdot, t)\right\rangle .
$$

Assim, para cada $k \in \mathbb{Z}$,

$$
G_{\tau}, T: X_{1} \rightarrow X_{2}
$$

são operadores lineares com $T \in \mathscr{L}\left(X_{1} ; X_{2}\right)$, onde

$$
\begin{gathered}
T u(\cdot, t) \doteq u(\cdot, t)_{\mid B_{R / 2}},|t| \leq R^{\prime} \\
X_{1 k}=\left\{v \in C^{0}\left(\left\{|t| \leq R^{\prime}\right\} ; H^{k}\left(\mathbb{R}^{m}\right)\right) ; \operatorname{supp}(v(\cdot, t)) \subset \bar{B}_{R^{\prime \prime}}\right\}
\end{gathered}
$$

e

$$
X_{2 k}=C^{0}\left(\left\{|t| \leq R^{\prime}\right\} ; H^{k}\left(B_{\frac{R}{2}}\right)\right)
$$

são espaços normados.

Proposição 7.2.11. $G_{\tau} \in \mathscr{L}\left(X_{1 k} ; X_{2 k}\right), \forall k \in \mathbb{N}$. 
Demonstração. Primeiramente, façamos o caso em que $k=0$, nesta situção temos

$$
H^{0}\left(\mathbb{R}^{m}\right)=L^{2}\left(\mathbb{R}^{m}\right)
$$

e assim $u(\cdot, t)$ é uma função em $L^{2}$. Note que este fato simplifica a expressão do operador, a saber

$$
G_{\tau} u(x, t)=(\tau / \pi)^{m / 2} \int_{\mathbb{R}^{m}} e_{\tau}\left(Z(x, t), x^{\prime}, t\right) u\left(x^{\prime}, t\right) h\left(x^{\prime}\right) \operatorname{det} Z_{x}\left(x^{\prime}, t\right) d x^{\prime} .
$$

Lembramos que

$$
\left|e_{\tau}\left(Z(x, t), x^{\prime}, t\right)\right|=\left|e^{-\tau\left[Z(x, t)-Z\left(x^{\prime}, t\right)\right]^{2}}\right| \leq e^{\frac{-3 \tau}{4}\left|x-x^{\prime}\right|^{2}} .
$$

Defina

$$
F_{\tau}(x) \doteq \tau^{m / 2} e^{\frac{-3 \tau|x|^{2}}{4}}, \tau>0, x \in \mathbb{R}^{m}
$$

Assim

$$
\left|G_{\tau} u(x, t)\right| \leq(\tau / \pi)^{m / 2} \int_{\mathbb{R}^{m}} e^{\frac{-3 \tau}{4}\left|x-x^{\prime}\right|^{2}}|u|\left(x^{\prime}, t\right)\left|h\left(x^{\prime}\right) \operatorname{det} Z_{x}\left(x^{\prime}, t\right)\right| d x^{\prime} \leq C F_{\tau} *|u|(x, t) .
$$

Como

$$
\left\|F_{\tau}\right\|_{L^{1}}=\tau^{m / 2} \int_{\mathbb{R}^{m}} e^{\frac{-3}{4}|x|^{2} \tau} d x=\int_{\mathbb{R}^{m}} e^{\frac{-3}{4}|x|^{2}} d x=\left\|F_{1}\right\|_{L^{1}},
$$

aplicando a desigualdade de Young em 7.7 obtemos

$$
\left\|G_{\tau} u(\cdot, t)\right\|_{L^{2}\left(B_{R / 2}\right)} \leq C\left\|F_{1}\right\|_{L^{1}}\|u(\cdot, t)\|_{L^{2}}=C_{0}\|u(\cdot, t)\|_{L^{2}},
$$

para qualquer $|t| \leq R^{\prime}$. Portanto

$$
\sup _{|t| \leq R^{\prime}}\left\|G_{\tau} u(\cdot, t)\right\|_{L^{2}\left(B_{R / 2}\right)} \leq C_{0} \sup _{|t| \leq R^{\prime}}\|u(\cdot, t)\|_{L^{2}}
$$

demonstrando o resultado para $k=0$.

Para $k=1$, basta encontrarmos uma constante $C_{0}$ tal que

$$
\sup _{|t| \leq R^{\prime}}\left\|\partial_{x_{j}} G_{\tau} u(\cdot, t)\right\|_{L^{2}\left(B_{R / 2}\right)} \leq C_{0} \sup _{|t| \leq R^{\prime}}\|u(\cdot, t)\|_{H^{1}}
$$

$j=1, \ldots, m$. Para tanto utilizaremos os campos $M_{k}$, visto que

$$
\partial_{x_{j}}=\frac{\partial}{\partial x_{j}}=\sum_{k=1}^{m} \frac{\partial Z_{k}}{\partial x_{j}} M_{k} .
$$

Tendo em vista que as funções $\mu_{k l}$ são suaves e $\bar{B}_{R^{\prime \prime}} \times \bar{B}_{R^{\prime}}$ é compacto, tem-se que tais funções são limitadas neste conjunto. Logo existe constante $M_{0}$ tal que

$$
\left\|M_{k} u(\cdot, t)\right\|_{L^{2}} \leq M_{0} \sum_{j=1}^{m}\left\|\partial_{x_{j}} u(\cdot, t)\right\|_{L^{2}} \leq M_{0}\|u(\cdot, t)\|_{H^{1}},
$$


e assim

$$
\left\|G_{\tau} M_{k} u(\cdot, t)\right\|_{L^{2}\left(B_{R / 2}\right)} \leq C_{0}\left\|M_{k} u(\cdot, t)\right\|_{L^{2}} \leq C_{0} M_{0}\|u(\cdot, t)\|_{H^{1}} .
$$

Além disso,

$$
M_{k} G_{\tau}=G_{\tau} M_{k}+\left[M_{k}, G_{\tau}\right]
$$

e

$$
\left[M_{k}, G_{\tau}\right] u(x, t)=(\tau / \pi)^{m / 2} \int_{\mathbb{R}^{m}} e_{\tau}\left(Z(x, t), x^{\prime}, t\right) u\left(x^{\prime}, t\right)\left(M_{k} h\right)\left(x^{\prime}\right) \operatorname{det} Z_{x}\left(x^{\prime}, t\right) d x^{\prime}
$$

Analogamente ao caso anterior temos

$$
\left|\left[M_{k}, G_{\tau}\right] u(x, t)\right| \leq C F_{\tau} *|u|(x, t),
$$

então

$$
\left\|\left[M_{k}, G_{\tau}\right] u(\cdot, t)\right\|_{L^{2}\left(B_{R / 2}\right)} \leq C\left\|F_{\tau}\right\|_{L^{1}}\|u(\cdot, t)\|_{L^{2}}=C_{0}\|u(\cdot, t)\|_{L^{2}} \leq C_{0}\|u(\cdot, t)\|_{H^{1}},
$$

para qualquer $|t| \leq R^{\prime}$. Juntandos os fatos, encontramos uma constante, que por simplicidade denotaremos por $C_{0}$, tal que

$$
\begin{aligned}
\sup _{|t| \leq R^{\prime}}\left\|M_{k} G_{\tau} u(\cdot, t)\right\|_{L^{2}\left(B_{R / 2}\right)} & \leq C_{0} \sup _{|t| \leq R^{\prime}}\left(\left\|\left[M_{k}, G_{\tau}\right] u(\cdot, t)\right\|_{L^{2}\left(B_{R / 2}\right)}+\left\|G_{\tau} M_{k} u(\cdot, t)\right\|_{L^{2}\left(B_{R / 2}\right)}\right) \leq \\
& \leq C_{0} \sup _{|t| \leq R^{\prime}}\|u(\cdot, t)\|_{H^{1}} .
\end{aligned}
$$

Agora observe que como $\left(\partial Z_{k} / \partial x_{j}\right)$ são funções suaves então são limitadas no compacto $\bar{B}_{R^{\prime \prime}} \times \bar{B}_{R^{\prime}}$, portanto existe $C_{\mu}>0$ tal que

$$
\left\|\partial_{j} G_{\tau} u(\cdot, t)\right\|_{L^{2}\left(B_{R / 2}\right)} \leq C_{\mu} \sum_{k=1}^{m}\left\|M_{k} G_{\tau} u(\cdot, t)\right\|_{L^{2}\left(B_{R / 2}\right)} \leq C_{\mu} C_{0} m\|u(\cdot, t)\|_{H^{1}} .
$$

Unindo o a expressão acima ao resultado obtido para o caso $k=0$, obtemos que existe contante $C_{1}>0$, tal que

$$
\sup _{\|t\| \leq R^{\prime}}\left\|G_{\tau} u(\cdot, t)\right\|_{H^{1}\left(B_{R / 2}\right)} \leq C_{1}\|u(\cdot, t)\|_{H^{1}}
$$

Seja $k_{0} \in \mathbb{N}$, lembramos que se

$$
M_{K_{J}} \doteq M_{k_{1}} \ldots M_{k_{j_{0}}}, 1 \leq j_{0} \leq k_{0}
$$

então

$$
\begin{aligned}
M_{K_{J}} G_{\tau} & =\left[M_{k_{1}}, G_{\tau}\right] M_{k_{2}} \ldots M_{k_{j_{0}}} \ldots+M_{k_{1}}\left[M_{k_{2}}, G_{\tau}\right] \ldots M_{k_{j_{0}}}+\ldots \\
& +M_{k_{1}} \ldots M_{k_{j_{0}-1}}\left[M_{j_{0}}, G_{\tau}\right]+G_{\tau} M_{K_{J}}
\end{aligned}
$$

Vamos estimar $\left\|M_{K_{J}} G_{\tau} u(\cdot, t)\right\|_{L^{2}\left(B_{R / 2}\right)}$. 
Para tanto, primeiramente provemos que para cada $\alpha \in \mathbb{Z}^{m}$ multi-índice existem funções

$$
g_{\alpha} \in C^{\infty}\left(\bar{B}_{R^{\prime}} \times \bar{B}_{R^{\prime}} \times \mathbb{R}^{m}\right)
$$

com

$$
\operatorname{supp}\left(g_{\alpha}(x, t, \cdot)\right) \subset \operatorname{supp}(h), \forall(x, t) \in \mathbb{R}^{m} \times B_{R^{\prime \prime}},
$$

e tais que a seguinte fórmula é verdadeira

$$
\partial^{\alpha}\left[M_{k}, G_{\tau}\right] u(x, t)=(\tau / \pi)^{m / 2} \int_{\mathbb{R}^{m}} e_{\tau}\left(Z(x, t), x^{\prime}, t\right) u\left(x^{\prime}, t\right) g_{\alpha}\left(x, x^{\prime}, t\right) d x^{\prime} .
$$

Provemos por indução. Se $|\alpha|=1$, então $\partial^{\alpha}=\partial_{x_{j}}$. Devido ao fato de

$$
e_{\tau}\left(Z(x, t), x^{\prime}, t\right) u\left(x^{\prime}, t\right)\left(M_{k} h\right)\left(x^{\prime}\right) \operatorname{det} Z_{x}\left(x^{\prime}, t\right)
$$

ter suporte compacto na variável $x^{\prime}$, tal função é uniformemente contínua e então podemos derivar sob o sinal de integração na fórmula de $\left[M_{k}, G_{\tau}\right] u$ :

$$
\partial_{x_{j}}\left[M_{k}, G_{\tau}\right] u(x, t)=(\tau / \pi)^{m / 2} \int_{\mathbb{R}^{m}} e_{\tau}\left(Z(x, t), x^{\prime}, t\right) u\left(x^{\prime}, t\right) g_{j}\left(x, t, x^{\prime}\right) d x^{\prime},
$$

onde

$$
g_{j}\left(x, t, x^{\prime}\right)=-2 \tau\left(M_{k} h\left(x^{\prime}\right) \operatorname{det} Z\left(x^{\prime}, t\right)\right) \sum_{l=1}^{m}\left(Z_{l}(x, t)-Z_{l}\left(x^{\prime}, t\right)\right) \partial_{x_{j}} Z_{l}(x, t) .
$$

Suponha que a fórmula vale para $|\alpha|=\alpha_{0}$. Vamos calcular $\partial_{x_{j}} \partial^{\alpha}\left[M_{k}, G_{\tau}\right] u$. Observe que por hipótese de indução

$$
e_{\tau}\left(Z(x, t), x^{\prime}, t\right) u\left(x^{\prime}, t\right) g_{\alpha}\left(x, t, x^{\prime}\right)
$$

tem suporte compacto na variável $x^{\prime}$, portanto podemos derivar sob o sinal de integração e encontrar

$$
(\tau / \pi)^{m / 2} \int_{\mathbb{R}^{m}} e_{\tau}\left(Z(x, t), x^{\prime}, t\right) u\left(x^{\prime}, t\right)\left(\partial_{x_{j}} g_{\alpha}\left(x, t, x^{\prime}\right)+g_{\alpha}\left(x, t, x^{\prime}\right) g_{j}\left(x, t, x^{\prime}\right)\right) d x^{\prime} .
$$

Como a função

$$
\partial_{x_{j}} g_{\alpha}\left(x, t, x^{\prime}\right)+g_{\alpha}\left(x, t, x^{\prime}\right) g_{j}\left(x, t, x^{\prime}\right)
$$

é suave e para cada $(x, t)$ possui suporte contido no suporte de $h$, fica provada a fórmula.

Utilizando a fórmula e observando que

$$
\operatorname{supp}\left(g_{\alpha}\right) \subset \bar{B}_{R^{\prime \prime}} \times \bar{B}_{R^{\prime \prime}} \times \operatorname{supp}(h),
$$

concluímos que existe constante $C_{\alpha}>0$ tal que

$$
\left|g_{\alpha}\left(x, t, x^{\prime}\right)\right| \leq C_{\alpha},
$$


para quaisquer $\left(x, t, x^{\prime}\right)$ em seu domínio. Dessa forma,

$$
\left|\partial^{\alpha}\left[M_{k}, G_{\tau}\right] u(x, t)\right| \leq C_{\alpha} \tau^{m / 2} \int_{\mathbb{R}^{m}} e^{-\tau \frac{3}{4}\left|x-x^{\prime}\right|^{2}}|u|\left(x^{\prime}, t\right) d x^{\prime}=C_{\alpha} F_{\tau} *|u|(x, t) .
$$

Pelo mesmo raciocínio usado anteriormente, tem-se que

$$
\left\|\partial^{\alpha}\left[M_{k}, G_{\tau}\right] u(\cdot, t)\right\|_{L^{2}\left(B_{R / 2}\right)} \leq C_{\alpha}\|u(\cdot, t)\|_{L^{2}}
$$

e portanto

$$
\left\|\left[M_{k}, G_{\tau}\right] u(\cdot, t)\right\|_{H^{|\alpha|}\left(B_{R / 2}\right)} \leq C_{\alpha}\|u(\cdot, t)\|_{L^{2}} .
$$

Agora afirmamos que, para cada $l_{1}, \ldots, l_{j_{0}}=1, \ldots, m$, existem funções suaves $a_{l_{1} \ldots l_{j_{0}}}, \ldots, a_{l_{1}}$ tais que

$$
M_{K_{J}}=\sum_{l_{1}, \ldots, l_{j_{0}}=1}^{m} a_{l_{1} \ldots l_{j_{0}}} \partial_{x_{l_{1}}} \ldots \partial_{x_{l_{j_{0}}}}+\ldots+\sum_{l_{1}=1}^{m} a_{l_{1}} \partial_{x_{l_{1}}}
$$

para qualquer $j_{0} \in \mathbb{N}$.

Para $j_{0}=1$ basta notar que

$$
M_{k}=\sum_{j=1}^{m} \mu_{k j} \partial_{x_{j}}
$$

Suponha a fórmula válida para $j_{0} \in \mathbb{N}$ e provemos para $j_{0}+1$. Assim

$$
\begin{array}{r}
M_{k}\left(M_{K_{J}}\right)=\left(\sum_{j=1}^{m} \mu_{k j} \partial_{x_{j}}\right)\left(\sum_{l_{1}, \ldots, l_{j_{0}}=1}^{m} a_{l_{1} \ldots l_{j_{0}}} \partial_{x_{l_{1}}} \ldots \partial_{x_{l_{j_{0}}}}+\ldots+\sum_{l_{1}=1}^{m} a_{l_{1}} \partial_{x_{l_{1}}}\right)= \\
=\sum_{j, l_{1}, \ldots, l_{j_{0}}=1}^{m} \mu_{k j} a_{j} a_{l_{1} \ldots l_{j_{0}}} \partial_{x_{j}} \partial_{x_{l_{1}}} \ldots \partial_{x_{l_{0}}}+\ldots+\sum_{l_{1}=1}^{m}\left(\sum_{j=1}^{m} \mu_{k j} \partial x_{j} a_{l_{1}}\right) \partial x_{l_{1}},
\end{array}
$$

ou seja,

$$
M_{k} M_{K_{J}}=\sum_{l_{1}, \ldots, l_{j_{0}}, l_{j_{0}+1}=1}^{m} \tilde{a}_{l_{1} \ldots l_{j_{0}}} \partial_{x_{l_{1}}} \ldots \partial_{x_{l_{j_{0}+1}}}+\ldots+\sum_{l_{1}=1}^{m} \tilde{a}_{l_{1}} \partial_{x_{l_{1}}},
$$

em que as seguintes funções

$$
\begin{aligned}
\tilde{a}_{l_{1}} & \doteq \sum_{l=1}^{m} \mu_{k l} \partial_{x_{l}} a_{l_{1}} \\
\tilde{a}_{l_{1}, \ldots, l_{j_{0}}} & \doteq \mu_{k j_{0}+1} a_{j_{0}+1} a_{l_{1} \ldots l_{j_{0}+1}} \\
\tilde{a}_{l_{1} \ldots, l_{j}} & \doteq \mu_{k l_{j}} a_{l_{1} \ldots l_{j-1}}+\sum_{l=1}^{m} \mu_{k l} \partial x_{l} a_{l_{1} \ldots l_{j}}, 2 \leq j \leq j_{0}
\end{aligned}
$$

são suaves e portanto a fórmula está provada.

Além disso, por estes coeficientes serem funções suaves segue que são limitadas no compacto $\bar{B}_{R^{\prime \prime}} \times \bar{B}_{R^{\prime \prime}}$ e assim existe constante $K_{\alpha}>0$ tal que

$$
\left|a_{l_{1} \ldots, l_{j}}(x, t)\right| \leq M_{\alpha}, 1 \leq|\alpha| .
$$


Agora provemos que, para cada $k_{0} \in \mathbb{N}$, existe constante $C_{k_{0}}>0$

$$
\sup _{\|t\| \leq R^{\prime}}\left\|G_{\tau} u(\cdot, t)\right\|_{H^{k_{0}}\left(B_{R / 2}\right)} \leq C_{k_{0}} \sup _{\|t\| \leq R^{\prime}}\|u(\cdot, t)\|_{H^{k_{0}}}
$$

Então, unindos os dois fatos

$$
\left\|M_{K_{J}} u(\cdot, t)\right\|_{L^{2}\left(B_{R / 2}\right)} \leq M_{j_{0}} \sum_{|\alpha| \leq j_{0}}\left\|\partial^{\alpha} u(\cdot, t)\right\|_{L^{2}} \leq M_{j_{0}}\|u(\cdot, t)\|_{H^{j_{0}}} .
$$

Por fim, afirmamos que se $\alpha_{0} \in \mathbb{N}$ então existe $C_{\alpha_{0}}>0$ constante tal que

$$
\left\|M_{K_{J}} u(\cdot, t)\right\|_{H^{\alpha_{0}\left(B_{R / 2}\right)}} \leq C_{\alpha_{0}}\|u(\cdot, t)\|_{H^{\alpha_{0}+j_{0}}} .
$$

De fato, provemos antes que para $\alpha \in \mathbb{Z}$ vale

$$
\partial^{\alpha} M_{K_{J}}=\sum_{l_{1}, \ldots, l_{j_{0}+|\alpha|}=1}^{m} a_{l_{1} \ldots l_{j_{0}}+|\alpha|} \partial_{x_{l_{1}}} \ldots \partial_{x_{l_{j_{0}}+|\alpha|}}+\ldots+\sum_{l_{1}=1}^{m} a_{l_{1}} \partial_{x_{l_{1}}} .
$$

Faremos a prova da fórmula acima por induçãao no comprimento de $\alpha$. Se $|\alpha|=1$, então

$$
\begin{array}{r}
\partial_{x_{l}} M_{K_{J}}=\sum_{l_{1}, \ldots, l_{j_{0}}=1}^{m} a_{l_{1} \ldots l_{j_{0}}+|\alpha|} \partial_{x_{l}} \partial_{x_{l_{1}}} \ldots \partial_{x_{l_{j_{0}}+|\alpha|}}+ \\
+\sum_{l_{1}, \ldots, l_{j_{0}}=1}^{m} \partial_{x_{l}} a_{l_{1} \ldots l_{j_{0}}+|\alpha|} \partial_{x_{l}} \partial_{x_{l_{1}}} \ldots \partial_{x_{l_{j_{0}}+|\alpha|}}+\ldots+\sum_{l_{1}=1}^{m} \partial_{x_{l}} a_{l_{1}} \partial_{x_{l_{1}}}
\end{array}
$$

Suponha que a fórmula é verdadeira para $|\alpha|$ e calculemos $\partial_{x_{l}} \partial^{\alpha} M_{K_{J}}$ :

$$
\begin{gathered}
\partial_{x_{l}} \partial^{\alpha} M_{K_{J}}=\sum_{l_{1}, \ldots, l_{j_{0}+|\alpha|}=1}^{m} a_{l_{1} \ldots l_{j_{0}}+|\alpha|} \partial_{x_{l}} \partial_{x_{l_{1}}} \ldots \partial_{x_{l_{j_{0}}+\alpha \mid}}+ \\
\sum_{l_{1}, \ldots, l_{j_{0}+|\alpha|}=1}^{m} \partial_{x_{l}} a_{l_{1} \ldots l_{j_{0}}+|\alpha|} \partial_{x_{l_{1}}} \ldots \partial_{x_{l_{j_{0}}+|\alpha|}}+\ldots+\sum_{l_{1}=1}^{m} \partial_{x_{l}} a_{l_{1}} \partial_{x_{l_{1}}}
\end{gathered}
$$

provando a fórmula.

Observe mais uma vez que os coeficientes da fórmula 7.8 são funções suaves e então são limitadas no compacto $\bar{B}_{R^{\prime \prime}} \times \bar{B}_{R^{\prime \prime}}$.

Em suma, concluímos que para $0 \leq|\alpha| \leq \alpha_{0}$ existe constante $C_{\alpha}>0$ tal que

$$
\left\|\partial^{\alpha} M_{K_{J}} u(\cdot, t)\right\|_{L^{2}\left(B_{R}\right)} \leq C_{\alpha}\|u(\cdot, t)\|_{H^{|\alpha|+j_{0}}} \leq C_{\alpha}\|u(\cdot, t)\|_{H^{\alpha_{0}+j_{0}}},
$$

$\log 0$

$$
\left\|M_{K_{J}} u(\cdot, t)\right\|_{H^{\alpha_{0}\left(B_{R}\right)}} \leq C_{\alpha}\|u(\cdot, t)\|_{H^{\alpha_{0}+j_{0}}} .
$$


Assim

$$
\begin{array}{r}
\left\|M_{k_{1}} \ldots M_{k_{j}}\left[M_{k_{j+1}}, G_{\tau}\right] M_{k_{j+2}} \ldots M_{k_{j}} u(\cdot, t)\right\|_{L^{2}\left(B_{R / 2}\right)} \leq \\
\leq C_{j}\left\|\left[M_{k_{j+1}}, G_{\tau}\right] M_{k_{j+2}} \ldots M_{k_{j_{0}}} u(\cdot, t)\right\|_{H^{j}\left(B_{R / 2}\right)} \leq \\
\leq C_{j+1} C_{j}\left\|M_{k_{j+2}} \ldots M_{k_{j_{0}}} u(\cdot, t)\right\|_{H^{j}} \leq C_{j+2} C_{j+1} C_{j}\|u(\cdot, t)\|_{H^{j}} .
\end{array}
$$

Daí, existe constante $C_{K_{J}}>0$ tal que

$$
\left\|M_{K_{J}} G_{\tau} u(\cdot, t)\right\|_{L^{2}\left(B_{R / 2}\right)} \leq C_{K_{J}}\|u(\cdot, t)\|_{H^{j_{0}}}, \forall 1 \leq j_{0} \leq k_{0}
$$

Finalmente, a seguinte fórmula nos fornece a última informação necessária para provarmos a proposição no caso $k_{0} \in \mathbb{N}$. Para $\alpha \in \mathbb{N}$, afirmamos que vale

$$
\partial^{\alpha}=\sum_{l_{1}, \ldots, l_{|\alpha|}}^{m} b_{l_{1} \ldots l_{|\alpha|}} M_{l_{1}} \ldots M_{l_{|\alpha|}}+\ldots+\sum_{l_{1}=1}^{m} b_{l_{1}} M_{l_{1}}
$$

onde $\partial^{\alpha}=\partial_{x_{1}}^{\alpha_{1}} \ldots \partial_{x_{m}}^{\alpha_{m}}$ e os coeficientes são funções suaves.

Novamente, a prova é dada por indução finita. Para $|\alpha|=1$ :

$$
\partial_{x_{l}}=\sum_{k=1}^{m} \frac{\partial Z_{k}}{\partial x_{l}} M_{k}
$$

Suponha a fórmula válida para $\alpha$ e calculemos $\partial_{x_{l}} \partial^{\alpha}$ :

$$
\begin{aligned}
& \partial_{x_{l}}\left(\sum_{l_{1}, \ldots, l_{|\alpha|}}^{m} b_{l_{1} \ldots l_{|\alpha|}} M_{l_{1}} \ldots M_{l_{|\alpha|}}+\ldots+\sum_{l_{1}=1}^{m} b_{l_{1}} M_{l_{1}}\right)= \\
& =\sum_{k, l_{1}, \ldots, l_{|\alpha|}}^{m} \partial_{x_{l}} Z_{k} b_{l_{1} \ldots l_{|\alpha|}} M_{k} M_{l_{1}} \ldots M_{l_{|\alpha|}}+ \\
& +\sum_{k, l_{1}, \ldots, l_{|\alpha|}}^{m}\left(\sum_{k=1}^{m} \partial_{x_{l}} Z_{k} M_{k} b_{l_{1} \ldots l_{|\alpha|}}\right) M_{l_{1}} \ldots M_{l_{|\alpha|}}+\ldots+ \\
& +\sum_{l_{1}=1}^{m}\left(\sum_{k=1}^{m} \partial_{x_{l}} Z_{k} M_{k} b_{l_{1}}\right) M_{l_{1}} .
\end{aligned}
$$

Logo se $|\alpha| \leq k_{0}$ :

$$
\begin{aligned}
\left\|\partial^{\alpha} G_{\tau} u(\cdot, t)\right\|_{L^{2}\left(B_{R}\right)} & \leq C_{\alpha} \sum_{l_{1}, \ldots, l_{|\alpha|}}\left\|M_{k_{|\alpha|}} u(\cdot, t)\right\|_{L^{2}\left(B_{R}\right)}+\ldots \\
& +C_{\alpha} \sum_{l_{1}=1}^{m}\left\|M_{l_{1}} u(\cdot, t)\right\|_{L^{2}\left(B_{R}\right)} \leq \\
& \leq \tilde{C}_{\alpha}\left(\|u(\cdot, t)\|_{H^{|\alpha|}}+\ldots+\|u(\cdot, t)\|_{H^{1}}\right) \leq \\
& \leq C\|u(\cdot, t)\|_{H^{k_{0}}},
\end{aligned}
$$

isto é,

$$
\left\|G_{\tau} u(\cdot, t)\right\|_{H^{k_{0}\left(B_{R / 2}\right)}} \leq C\|u(\cdot, t)\|_{H^{k_{0}}} .
$$


Como as constantes encontradas durante a demonstração não dependem da variável $t$, concluimos que

$$
\sup _{|t| \leq R^{\prime \prime}}\left\|G_{\tau} u(\cdot, t)\right\|_{H^{k_{0}\left(B_{R}\right)}} \leq C \sup _{|t| \leq R^{\prime \prime}}\|u(\cdot, t)\|_{H^{k_{0}}} .
$$

Proposição 7.2.12. $G_{\tau} \in \mathscr{L}\left(X_{1-k} ; X_{2-k}\right), \forall k \in \mathbb{N}$.

Demonstração. Inicialmente, se $a, b \in C^{\infty}\left(\mathbb{R}^{m}\right)$ uma delas com suporte compacto denotamos

$$
\langle a, b\rangle \doteq \int_{\mathbb{R}^{m}} a(x) b(x) \operatorname{det} Z_{x}(x, t) d x
$$

e para $u \in C^{0}\left(\bar{B}_{R^{\prime}} ; H^{-k}\left(\mathbb{R}^{m}\right)\right)$ defina $G_{\tau}^{\prime} u(x, t) \doteq h(x) G_{\tau} u(x, t)$.

Assim, como

$$
e_{\tau}\left(Z(x, t), x^{\prime}, t\right)=e^{-\tau\left[Z(x, t)-Z\left(x^{\prime}, t\right)\right]^{2}}=e^{-\tau\left[Z\left(x^{\prime}, t\right)-Z(x, t)\right]^{2}}=e_{\tau}\left(Z\left(x^{\prime}, t\right), x, t\right),
$$

então para $v, w \in C_{c}^{\infty}\left(\mathbb{R}^{m}\right)$ tem-se que $\left\langle G_{\tau}^{\prime} v, w\right\rangle$ é dado por

$$
\begin{array}{r} 
\\
\int_{\mathbb{R}^{m}} h(x) \int_{\mathbb{R}^{m}} e_{\tau}\left(Z(x, t), x^{\prime}, t\right) h\left(x^{\prime}\right) v\left(x^{\prime}\right) \operatorname{det} Z_{x}\left(x^{\prime}, t\right) d x^{\prime} w(x) \operatorname{det} Z_{x}(x, t) d x= \\
=\int_{\mathbb{R}^{m}} h(x) \int_{\mathbb{R}^{m}} e_{\tau}\left(Z\left(x^{\prime}, t\right), x, t\right) h\left(x^{\prime}\right) v\left(x^{\prime}\right) \operatorname{det} Z_{x}\left(x^{\prime}, t\right) d x^{\prime} w(x) \operatorname{det} Z_{x}(x, t) d x= \\
=\int_{\mathbb{R}^{m}} h\left(x^{\prime}\right) G_{\tau}^{\prime} w\left(x^{\prime}\right) v\left(x^{\prime}\right) \operatorname{det} Z_{x}\left(x^{\prime}, t\right) d x^{\prime}=\left\langle v, G_{\tau}^{\prime} w\right\rangle .
\end{array}
$$

Logo

$$
\left\langle G_{\tau}^{\prime} u(\cdot, t), w\right\rangle=\left\langle u(\cdot, t), G_{\tau}^{\prime} w\right\rangle, w \in C_{c}^{\infty}\left(\mathbb{R}^{m}\right) .
$$

Como $C_{c}^{\infty}\left(\mathbb{R}^{m}\right)$ é denso em $H^{k}\left(B_{R}\right)$, obtém-se que

$$
\left\langle G_{\tau}^{\prime} u(\cdot, t), w\right\rangle=\left\langle u(\cdot, t), G_{\tau}^{\prime} w\right\rangle, w \in H^{k}\left(B_{R}\right)
$$

então $G_{\tau}^{\prime} u(\cdot, t) \in\left(H^{k}\left(B_{R}\right)\right)^{*}=H^{-k}\left(B_{R}\right)$.

Observação 7.2.13. Ressaltamos que se $f \in H^{-k}$ então

$$
\|f\|_{-k}=\sup _{w \in H^{k},\|w\|_{k} \leq 1}|\langle f, w\rangle|
$$

Porém, dada $w \in H^{k}$ existe uma sequência $\left(\psi_{l}\right)_{l}$ de funções suaves com suporte compacto que convergem para w na norma de $H^{k}$. Assim existe $l_{0}$ tal que se $l \geq l_{0}$ vale

$$
\left\|w-\psi_{l}\right\|_{k}<\frac{1}{2}
$$

Então

$$
\left\|\psi_{l}\right\|_{k} \leq\|w\|_{k}+\left\|w-\psi_{l}\right\|_{k}<\frac{3}{2}
$$


para $l \geq l_{0}$ e $0<\|w\|_{k} \leq 1$. Considere a subsequência $\left(\psi_{l}\right)_{l \geq l_{0}}$

Enfim, temos que

$$
\begin{aligned}
|\langle f, w\rangle|=\lim _{l \rightarrow \infty}\left|\left\langle f, \phi_{l}\right\rangle\right| & \leq \lim _{l \rightarrow \infty} \frac{3}{2}\left|\left\langle f, \psi_{l} /\left(\left\|\psi_{l}\right\|_{k}\right)\right\rangle\right| \leq \\
\leq \lim _{l \rightarrow \infty} \frac{3}{2} \sup _{\psi \in C_{c}^{\infty}\left(\mathbb{R}^{m}\right),\|\psi\|_{k} \leq 1}|\langle f, \psi\rangle| & =\frac{3}{2} \sup _{\psi \in C_{c}^{\infty}\left(\mathbb{R}^{m}\right),\|\psi\|_{k} \leq 1}|\langle f, \psi\rangle| .
\end{aligned}
$$

Aplicando o que foi concluido na observação acima ao nosso caso, encontramos

$$
\begin{aligned}
\left\|G_{\tau}^{\prime} u(\cdot, t)\right\|_{-k} & \leq \frac{3}{2} \sup _{\psi \in C_{c}^{\infty}\left(\mathbb{R}^{m}\right),\|\psi\|_{k} \leq 1}\left|\left\langle G_{\tau}^{\prime} u(\cdot, t), \psi\right\rangle\right|= \\
& =\frac{3}{2} \sup _{\psi \in C_{c}^{\infty}\left(\mathbb{R}^{m}\right),\|\psi\|_{k} \leq 1}\left|\left\langle u(\cdot, t), G_{\tau}^{\prime} \psi\right\rangle\right| \leq \\
& \leq \frac{3}{2} \sup _{\psi \in C_{c}^{\infty}\left(\mathbb{R}^{m}\right),\|\psi\|_{k} \leq 1}\|u(\cdot, t)\|_{-k}\left\|G_{\tau}^{\prime} \psi\right\|_{k} \leq \\
& \leq C \sup _{\psi \in C_{c}^{\infty}\left(\mathbb{R}^{m}\right),\|\psi\|_{k} \leq 1}\|u(\cdot, t)\|_{-k}\left\|G_{\tau} \psi\right\|_{k} \\
& \leq C \sup _{\psi \in C_{c}^{\infty}\left(\mathbb{R}^{m}\right),\|\psi\|_{k} \leq 1}\|u(\cdot, t)\|_{-k}\|\psi\|_{k} \leq C\|u(\cdot, t)\|_{-k} .
\end{aligned}
$$

Agora note que se $\chi_{1} \in C_{c}^{\infty}\left(\mathbb{R}^{m}\right)$ e $\chi_{2} \in C_{c}^{\infty}\left(\mathbb{R}^{n}\right)$ com $\chi_{1}=1$ numa vizinhança de $\bar{B}_{R^{\prime}}$, com suporte contido em $B_{R^{\prime \prime}}, \chi_{2}=1$ numa vizinhança de $\bar{B}_{R^{\prime}}$, com suporte contido em $B_{R^{\prime \prime}}$, e $\hat{u} \doteq \chi_{1} \chi_{2} u \in X_{1}$, então

$$
\left\|G_{\tau} u-T u\right\|_{X_{2}}=\left\|G_{\tau} \hat{u}-T \hat{u}\right\|_{X_{2}} .
$$

Portanto, segue das proposições a respeito da densidade e continuidade do operador $G_{\tau}$ que existe sequência $\left(\psi_{l}\right)_{l}$ tal que

$$
\left\|\psi_{l}-\hat{u}\right\|_{X_{1}} \rightarrow 0, l \rightarrow \infty
$$

então

$$
\begin{aligned}
\left\|G_{\tau} \hat{u}-T \hat{u}\right\|_{X_{2}} & \leq\left\|G_{\tau}\left(\hat{u}-\psi_{l}\right)\right\|_{X_{2}}+\left\|G_{\tau} \psi_{l}-T \psi_{l}\right\|_{X_{2}}+\left\|T\left(\hat{u}-\psi_{l}\right)\right\|_{X_{2}} \leq \\
& \leq C\left\|\hat{u}-\psi_{l}\right\|_{X_{1}}+\left\|G_{\tau} \psi_{l}-T \psi_{l}\right\|_{X_{2}},
\end{aligned}
$$

para todo $l \in \mathbb{N}$ e $\tau>0$.

Fazendo $\tau \rightarrow+\infty$ :

$$
\varlimsup_{\tau \rightarrow+\infty}\left\|G_{\tau} \hat{u}-T \hat{u}\right\|_{X_{2}} \leq C\left\|\hat{u}-\psi_{l}\right\|_{X_{1}}, \forall l \in \mathbb{N},
$$

finalmente fazendo $l \rightarrow \infty$ :

$$
\varlimsup_{\tau \rightarrow+\infty}\left\|G_{\tau} \hat{u}-T \hat{u}\right\|_{X_{2}}=0 .
$$

Reunindo os fatos, concluimos que

$$
\lim _{\tau \rightarrow+\infty}\left\|G_{\tau} u-T u\right\|_{X_{2}}=\lim _{\tau \rightarrow+\infty}\left\|G_{\tau} \hat{u}-T \hat{u}\right\|_{X_{2}}=0 .
$$




\subsubsection{Teorema de Baouendi-Treves}

Teorema 7.2.14 (Fórmula Distribucional de Baouendi-Treves). Sejam $\Omega \subset \mathbb{R}^{N}$ um aberto que contém a origem e $\mathcal{L}$ uma estrutura localmente integrável sobre $\Omega$, em que

$$
\mathcal{L}_{p}^{\perp}=\left[d Z_{1 p}, \ldots, d Z_{m p}\right], \forall p \in \Omega
$$

e $Z_{1}, \ldots, Z_{m} \in C^{\infty}(\Omega)$. Nessas condições, existem abertos $U$ e $W$ que contêm a origem, $U \subset \bar{U} \subset W \subset \Omega$ tais que se $u \in \mathscr{D}^{\prime}(W)$ satisfaz

$$
\mathcal{L} u=0,
$$

então existe uma sequência de polinômios $P_{l} \circ Z$ que converge para u na topologia de $\mathscr{D}^{\prime}(U)$.

Demonstração. Primeiramente visto que o resultado é local pelo que foi feito na seção anterior podemos concluir a convergência de $G_{\tau} u$ para $u$ no caso distribucional. Assim, para obtermos o resultado no caso distribucional basta definirmos $E_{\tau} u$ e mostrarmos que o resto $R_{\tau} u$ converge para zero em seus respectivos espaços.

Analogamente a definição do operador $G_{\tau}$, definimos

$$
E_{\tau} u(x, t) \doteq\left\langle u(\cdot, 0),(\tau / \pi)^{m / 2} e^{-\tau[Z(x, t)-Z(\cdot, 0)]^{2}} h(\cdot) \operatorname{det} Z_{x}(\cdot, 0)\right\rangle
$$

e

$$
R_{\tau} u(x, t) \doteq G_{\tau} u(x, t)-E_{\tau}(x, t) .
$$

Observação 7.2.15. Sendo $u \in C^{\infty}\left(\left\{|t| \leq R^{\prime}\right\} ; \mathscr{D}\left(B_{R^{\prime}}\right)\right)$, para $\phi \in C^{\infty}\left(\mathbb{R}^{N}\right)$ existe compacto $K \subset \mathbb{R}^{m}$ tal que $\phi(\cdot, t) \in C^{\infty}(K), \forall|t| \leq R^{\prime}$. Nestas condições

$$
v(t) \doteq\langle u(\cdot, t), \phi(\cdot, t)\rangle,|t| \leq R^{\prime}
$$

é uma função $C^{1}$.

De fato,

$$
\begin{aligned}
\frac{v\left(t_{0}+h e_{j}\right)-v\left(t_{0}\right)}{h} & =\frac{1}{h}\left\langle u\left(\cdot, t_{0}+h e_{j}\right)-u\left(\cdot, t_{0}\right), \phi\left(\cdot, t_{0}\right)\right\rangle+ \\
& +\left\langle u\left(\cdot, t_{0}+h e_{j}\right), \frac{\partial \phi}{\partial t_{j}}\left(\cdot, t_{0}\right)\right\rangle+\left\langle u\left(\cdot, t_{0}+h e_{j}\right), \frac{r_{\phi}}{h} \cdot\right\rangle .
\end{aligned}
$$

Note que

$$
\left(u\left(\cdot, t_{0}+h e_{j}\right)\right)_{|h|<\delta} \subset \mathscr{L}\left(C_{c}^{\infty}(K) ; \mathbb{C}\right)
$$

com

$$
u\left(\cdot, t_{0}+h e_{j}\right) \rightarrow u\left(\cdot, t_{0}\right), \delta \rightarrow 0 .
$$


Pelo Teorema de Banach-Steinhauss para espaços de Fréchet existem $\delta^{\prime}>0$ e $M>0$ com $B_{\delta^{\prime}} \subset C_{c}^{\infty}(K)$ tal que

$$
\left|\left\langle u\left(\cdot, t_{0}+h e_{j}\right), \psi\right\rangle\right|<M, \forall|h|<\delta .
$$

Logo, dado $\varepsilon>0$, existe $0<\delta^{\prime \prime}<\min \left\{\delta, \delta^{\prime}\right\}$ tal que se $|h|<\delta^{\prime \prime}$ então

$$
\frac{r_{\phi}}{h} \in B_{\delta^{\prime}}
$$

$e$

$$
\left|\left\langle u\left(\cdot, t_{0}+h e_{j}\right), \frac{r_{\phi}}{h} \cdot\right\rangle\right|<\varepsilon .
$$

Calculemos agora a expressão do resto. Considere

$$
\tilde{G}_{\tau} u\left(\zeta, t^{\prime}\right) \doteq\left\langle u\left(\cdot, t^{\prime}\right), e_{\tau}\left(\zeta, \cdot, t^{\prime}\right) h(\cdot) \operatorname{det} Z_{x}\left(\cdot, t^{\prime}\right)\right\rangle .
$$

Assim

$$
\begin{aligned}
\frac{\partial \tilde{G}_{\tau} u}{\partial t_{j}}\left(Z(x, t), t^{\prime}\right) & =\left\langle\frac{\partial u}{\partial t_{j}}\left(\cdot, t^{\prime}\right), e_{\tau}\left(Z(x, t), \cdot, t^{\prime}\right) h(\cdot) \operatorname{det} Z_{x}\left(\cdot, t^{\prime}\right)\right\rangle+ \\
& +\left\langle u\left(\cdot, t^{\prime}\right), \frac{\partial\left(e_{\tau} h\right)}{\partial t_{j}}\left(Z(x, t), \cdot, t^{\prime}\right) \operatorname{det} Z_{x}\left(\cdot, t^{\prime}\right)\right\rangle+ \\
& +\left\langle u\left(\cdot, t^{\prime}\right), e_{\tau}\left(Z(x, t), \cdot, t^{\prime}\right) h(\cdot) \frac{\partial \operatorname{det} Z_{x}}{\partial t_{j}}\left(\cdot, t^{\prime}\right)\right\rangle .
\end{aligned}
$$

Como

$$
\frac{\partial}{\partial t_{j}}=L_{j}-\sum_{k=1}^{m} \lambda_{j k} \frac{\partial}{\partial x_{k}}
$$

tem-se que

$$
\begin{array}{r}
\left\langle\frac{\partial u}{\partial t_{j}}\left(\cdot, t^{\prime}\right), e_{\tau}\left(Z(x, t), \cdot, t^{\prime}\right) h(\cdot) \operatorname{det} Z_{x}\left(\cdot, t^{\prime}\right)\right\rangle=- \\
-\sum_{k=1}^{m}\left\langle\left(\lambda_{j k} \frac{\partial u}{\partial x_{k}}\left(\cdot, t^{\prime}\right)\right), e_{\tau}\left(Z(x, t), \cdot, t^{\prime}\right) h(\cdot) \operatorname{det} Z_{x}\left(\cdot, t^{\prime}\right)\right\rangle= \\
=\sum_{k=1}^{m}\left\langle u\left(\cdot, t^{\prime}\right), \frac{\partial\left(e_{\tau} h\right)}{\partial x_{k}}\left(Z(x, t), \cdot, t^{\prime}\right)\left(\lambda_{j k} \operatorname{det} Z_{x}\right)\left(\cdot, t^{\prime}\right)\right\rangle+ \\
+\left\langle u\left(\cdot, t^{\prime}\right), e_{\tau}\left(Z(x, t), \cdot, t^{\prime}\right) h(\cdot) \frac{\partial\left(\lambda_{j k} \operatorname{det} Z_{x}\right)}{\partial x_{k}}\left(\cdot, t^{\prime}\right)\right\rangle,
\end{array}
$$

e

$$
\begin{array}{r}
\left\langle u\left(\cdot, t^{\prime}\right), \frac{\partial\left(e_{\tau} h\right)}{\partial t_{j}}\left(Z(x, t), \cdot, t^{\prime}\right) \operatorname{det} Z_{x}\left(\cdot, t^{\prime}\right)\right\rangle= \\
=\left\langle u\left(\cdot, t^{\prime}\right), e_{\tau}\left(Z(x, t), \cdot, t^{\prime}\right) L_{j}(h)(\cdot) \operatorname{det} Z_{x}\left(\cdot, t^{\prime}\right)\right\rangle- \\
-\sum_{k=1}^{m}\left\langle u(\cdot, t), \lambda_{j k}(\cdot) \operatorname{det} Z_{x}(\cdot, t) \frac{e_{\tau} h}{\partial x_{k}}\left(Z(x, t), \cdot, t^{\prime}\right)\right\rangle,
\end{array}
$$


$\log 0$

$$
\frac{\partial \tilde{G}_{\tau} u}{\partial t_{j}}\left(Z(x, t), t^{\prime}\right)=\left\langle u\left(\cdot, t^{\prime}\right),\left(e_{\tau} L_{j}(h) \operatorname{det} Z_{x}\right)\left(Z(x, t), \cdot, t^{\prime}\right),\right\rangle
$$

pois

$$
e_{\tau} h\left(\frac{\partial \operatorname{det} Z_{x}}{\partial t_{j}}+\sum_{k=1}^{m} \frac{\partial\left(\lambda_{j k} \operatorname{det} Z_{x}\right)}{\partial x_{k}}\right)=0
$$

Defina $g\left(t^{\prime}\right) \doteq \tilde{G}_{\tau} u\left(Z(x, t), t^{\prime}\right)$, pelo teorema de Stokes segue que

$$
g(t)-g(0)=\int_{[0, t]} d g=\int_{[0, t]} \sum_{j=1}^{m} \frac{\partial g}{\partial t_{j}}\left(t^{\prime}\right) d t_{j}^{\prime} .
$$

Além disso,

$$
\begin{aligned}
& G_{\tau} u(x, t)=(\tau / \pi)^{m / 2} \tilde{G}_{\tau} u(Z(x, t), t)=(\tau / \pi)^{m / 2} g(t) \\
& E_{\tau} u(x, t)=(\tau / \pi)^{m / 2} \tilde{G}_{\tau} u(Z(x, t), 0)=(\tau / \pi)^{m / 2} g(0)
\end{aligned}
$$

e

$$
\frac{\partial g}{\partial t_{j}}\left(t^{\prime}\right)=\frac{\partial \tilde{G}_{\tau} u}{\partial t_{j}}\left(Z(x, t), t^{\prime}\right)
$$

obtemos que

$$
R_{\tau} u(x, t)=\int_{[0, t]} \sum_{j=1}^{m} r_{j}\left(x, t, t^{\prime}, \tau\right) d t_{j}^{\prime},
$$

em que

$$
r_{j}\left(x, t, t^{\prime}, \tau\right)=\left(\frac{\tau}{\pi}\right)^{m / 2} \int_{\mathbb{R}^{m}} e^{-\tau\left[Z(x, t)-Z\left(x^{\prime}, t^{\prime}\right)\right]^{2}} u\left(x^{\prime}, t^{\prime}\right) L_{j}(h)\left(x^{\prime}\right) \operatorname{det} Z_{x}\left(x^{\prime}, t^{\prime}\right) d x^{\prime} .
$$

Agora vamos estimar as expressões acima. Seja $\chi \in C_{c}^{\infty}\left(\mathbb{R}^{m}\right)$ que vale 1 numa vizinhança de $\bar{B}_{R / 4}$ e tem suporte contido em $B_{R / 2}$. Nestas condições, temos $\chi L_{j} h=L_{j} h$.

Note que para cada $k \in \mathbb{N}, t^{\prime} \in[0, t]$, tem-se

$$
L_{j} h u\left(\cdot, t^{\prime}\right) \operatorname{det} Z_{x}\left(\cdot, t^{\prime}\right)=\chi\left(1-\Delta_{x^{\prime}}\right)^{k}\left(1-\Delta_{x^{\prime}}\right)^{-k}\left(L_{j} h u\left(\cdot, t^{\prime}\right) \operatorname{det} Z_{x}\left(\cdot, t^{\prime}\right)\right) .
$$

Defina

$$
v_{j}\left(\cdot, t^{\prime}\right) \doteq\left(1-\Delta_{x^{\prime}}\right)^{-k}\left(L_{j} h u\left(\cdot, t^{\prime}\right) \operatorname{det} Z_{x}\left(\cdot, t^{\prime}\right)\right),
$$

e observe que

$$
L_{j} h u\left(\cdot, t^{\prime}\right) \operatorname{det} Z_{x}\left(\cdot, t^{\prime}\right) \in \mathscr{E}^{\prime}\left(\mathbb{R}^{m}\right) .
$$

Como

$$
\left(1-\Delta_{x^{\prime}}\right)^{-k}: H^{s}\left(\mathbb{R}^{m}\right) \rightarrow H^{s+2 k}\left(\mathbb{R}^{m}\right)
$$

é um operador linear contínuo e

$$
H^{s+2 k}\left(\mathbb{R}^{m}\right) \subset L^{\infty}\left(\mathbb{R}^{m}\right) \cap C^{0}\left(\mathbb{R}^{m}\right)
$$


se $s+2 k>m / 2$, então conseguimos encontrar $k \in \mathbb{N}$ tal que

$$
v_{j}\left(\cdot, t^{\prime}\right) \in L^{\infty}\left(\mathbb{R}^{m}\right) \cap C^{0}\left(\mathbb{R}^{m}\right) .
$$

Assim

$$
r_{j}\left(x, t, t^{\prime}, \tau\right)=\left(\frac{\tau}{\pi}\right)^{m / 2} \int_{\mathbb{R}^{m}} \chi\left(x^{\prime}\right) e^{-\tau\left[Z(x, t)-Z\left(x^{\prime}, t^{\prime}\right)\right]^{2}}\left(1-\Delta_{x^{\prime}}\right)^{k}\left(v_{j}\left(x^{\prime}, t^{\prime}\right)\right) d x^{\prime},
$$

integrando por partes obtemos

$$
r_{j}\left(x, t, t^{\prime}, \tau\right)=\left(\frac{\tau}{\pi}\right)^{m / 2} \int_{\mathbb{R}^{m}} v_{j}\left(x^{\prime}, t^{\prime}\right)\left(1-\Delta_{x^{\prime}}\right)^{k}\left(\chi\left(x^{\prime}\right) e^{-\tau\left[Z(x, t)-Z\left(x^{\prime}, t^{\prime}\right)\right]^{2}}\right) d x^{\prime},
$$

Analogamente ao caso clássico, tem-se que existe $g \in C^{\infty}\left(V \times \mathbb{R}^{m} \times[0, t]\right)$ função suave com suporte compacto na variável $x^{\prime}$ tal que

$$
\left|\left(1-\Delta_{x^{\prime}}\right)^{k}\left(\chi\left(x^{\prime}\right) e^{-\tau\left[Z(x, t)-Z\left(x^{\prime}, t^{\prime}\right)\right]^{2}}\right)\right| \leq e^{-\tau C} \tau^{2 k} g\left(x, t, x^{\prime}, t\right) .
$$

Dessa maneira,

$$
V \times[0, t] \ni\left(x, t, t^{\prime}\right) \mapsto \int_{\mathbb{R}^{m}}\left|v_{j}\left(x^{\prime}, t^{\prime}\right) g\left(x, t, x^{\prime}, t\right)\right| d x^{\prime}<\infty
$$

é uma função contínua então existe constante $M>0$ tal que

$$
\int_{\mathbb{R}^{m}}\left|v_{j}\left(x^{\prime}, t^{\prime}\right) g\left(x, t, x^{\prime}, t\right)\right| d x^{\prime} \leq M,
$$

para quaisquer $|x| \leq R / 2,\left|t^{\prime}\right| \leq|t| \leq R$.

Finalmente

$$
\left|r_{j}\left(x, t, t^{\prime}, \tau\right)\right| \leq \frac{\tau^{2 k+m / 2}}{\pi^{m / 2}} M e^{-C \tau} \rightarrow 0,
$$

uniformemente quanndo $\tau \rightarrow+\infty$ e então

$$
\begin{aligned}
\lim _{\tau \rightarrow+\infty}\left|R_{\tau} u(x, t)\right| & \leq \lim _{\tau \rightarrow+\infty} \int_{[0, t]} \sum_{j=1}^{m}\left|r_{j}\left(x, t, t^{\prime}, \tau\right)\right| d t_{j}^{\prime}= \\
& =\int_{[0, t]} \sum_{j=1}^{m} \lim _{\tau \rightarrow+\infty}\left|r_{j}\left(x, t, t^{\prime}, \tau\right)\right| d t_{j}^{\prime}=0 .
\end{aligned}
$$

Observe que da forma como definimos $E_{\tau} u$, mesmo quando $u$ é uma distribuição, esta função é suave nas variáveis $(x, t)$. Sendo assim, como no nosso caso

$$
u \in X_{1 k}
$$

para algum $k \in \mathbb{Z}$, temos que existe sequência de polinômios $P_{l}$ que aproxima a função $e^{-\tau[\zeta]^{2}}$ na topologia de $C^{\infty}$ no conjunto

$$
\left\{\tau^{1 / 2}\left(Z(x, t)-Z\left(x^{\prime}, 0\right)\right) ;|x|,\left|x^{\prime}\right| \leq R / 2,|t| \leq R\right\}
$$


em que

$$
P_{l}\left(Z(x, t)-Z\left(x^{\prime}, 0\right)\right)=\sum_{|\alpha|=0}^{l} C\left(x^{\prime}\right)(Z(x, t))^{\alpha}
$$

e assim

$$
\begin{array}{r}
\sup _{|t| \leq R}\left\|E_{\tau} u(\cdot, t)-(\tau / \pi)^{m / 2}\left\langle u(\cdot, t), P_{l}(Z(x, t)-Z(\cdot, 0)) h(\cdot) \operatorname{det} Z_{x}(\cdot, 0)\right\rangle\right\|_{k}= \\
=\sup _{|t| \leq R} \|(\tau / \pi)^{m / 2}\left\langle u(\cdot, t),\left(e^{-\tau[Z(x, t)-Z(\cdot, 0)]^{2}}-P_{l}(Z(x, t)-Z(\cdot, 0))\right) h(\cdot) \operatorname{det} Z_{x}(\cdot, t) \|_{k}\right.
\end{array}
$$

converge para zero quando $l \rightarrow \infty$.

Logo

$$
\begin{array}{r}
\sup _{|t| \leq R}\left\|u(\cdot, t)-(\tau / \pi)^{m / 2} \sum_{|\alpha|=0}^{l}(Z(\cdot, t))^{\alpha}\left\langle u(\cdot, t), C_{\alpha}(\cdot) h(\cdot) \operatorname{det} Z_{x}(\cdot, t)\right\rangle\right\|_{k} \leq \\
\leq \sup _{|t| \leq R}\left\|u(\cdot, t)-G_{\tau} u(\cdot, t)\right\|_{k}+\sup _{|t| \leq R}\left\|R_{\tau} u(\cdot, t)\right\|_{k}+ \\
+\sup _{|t| \leq R}\left\|E_{\tau} u(\cdot, t)-(\tau / \pi)^{m / 2}\left\langle u(\cdot, t), P_{l}(Z(x, t)-Z(\cdot, 0)) h(\cdot) \operatorname{det} Z_{x}(\cdot, 0)\right\rangle\right\|_{k},
\end{array}
$$

fazendo $l \rightarrow \infty$ obtemos

$$
\begin{aligned}
\lim _{l \rightarrow \infty} \sup _{|t| \leq R} \| u(\cdot, t)-(\tau / \pi)^{m / 2} & \sum_{|\alpha|=0}^{l}(Z(\cdot, t))^{\alpha}\left\langle u(\cdot, t), C_{\alpha}(\cdot) h(\cdot) \operatorname{det} Z_{x}(\cdot, t)\right\rangle \|_{k} \leq \\
& \leq \sup _{|t| \leq R}\left\|u(\cdot, t)-G_{\tau} u(\cdot, t)\right\|_{k}+\sup _{|t| \leq R}\left\|R_{\tau} u(\cdot, t)\right\|_{k},
\end{aligned}
$$

e finalmente fazendo $\tau \rightarrow+\infty$

$$
\lim _{\tau \rightarrow+\infty} \sup _{|t| \leq R}\left\|u(\cdot, t)-(\tau / \pi)^{m / 2} \sum_{|\alpha|=0}^{\infty}(Z(\cdot, t))^{\alpha}\left\langle u(\cdot, t), C_{\alpha}(\cdot) h(\cdot) \operatorname{det} Z_{x}(\cdot, t)\right\rangle\right\|_{k}=0 .
$$





\section{Referências}

[1] S. Berhanu, P. Cordaro and J. Hounie, An Introduction to Involutive Structures, Cambridge University Press, 2008. Citado 2 vezes nas páginas 11 e 13.

[2] Hormander, L. V., Linear Partial Differential Operators, Springer-Verlag, New York 1963. Citado 3 vezes nas páginas 11, 13 e 119.

[3] G.B. Folland, Real Analysis, Modern Techniques and Their Applications. 2nd ed. Wiley Interscience 1999. Citado na página 112.

[4] F. Treves, Topological Vector Spaces, Distributions and Kernels. Academic Press, New York 1967. Citado 2 vezes nas páginas 11 e 13.

[5] F. Treves, Approximation and representation of functions and distributions annihilated by systems of complex vector fields. Centre Math. École Polytechnique, 1981. Citado na página 121.

[6] E. Stein, R. Shakarchi, Complex Analysis. Princeton University Press, 2003. Citado na página 99 . 